\title{
Visual grading evaluation of reconstruction methods and dose optimisation in abdominal Computed Tomography
}

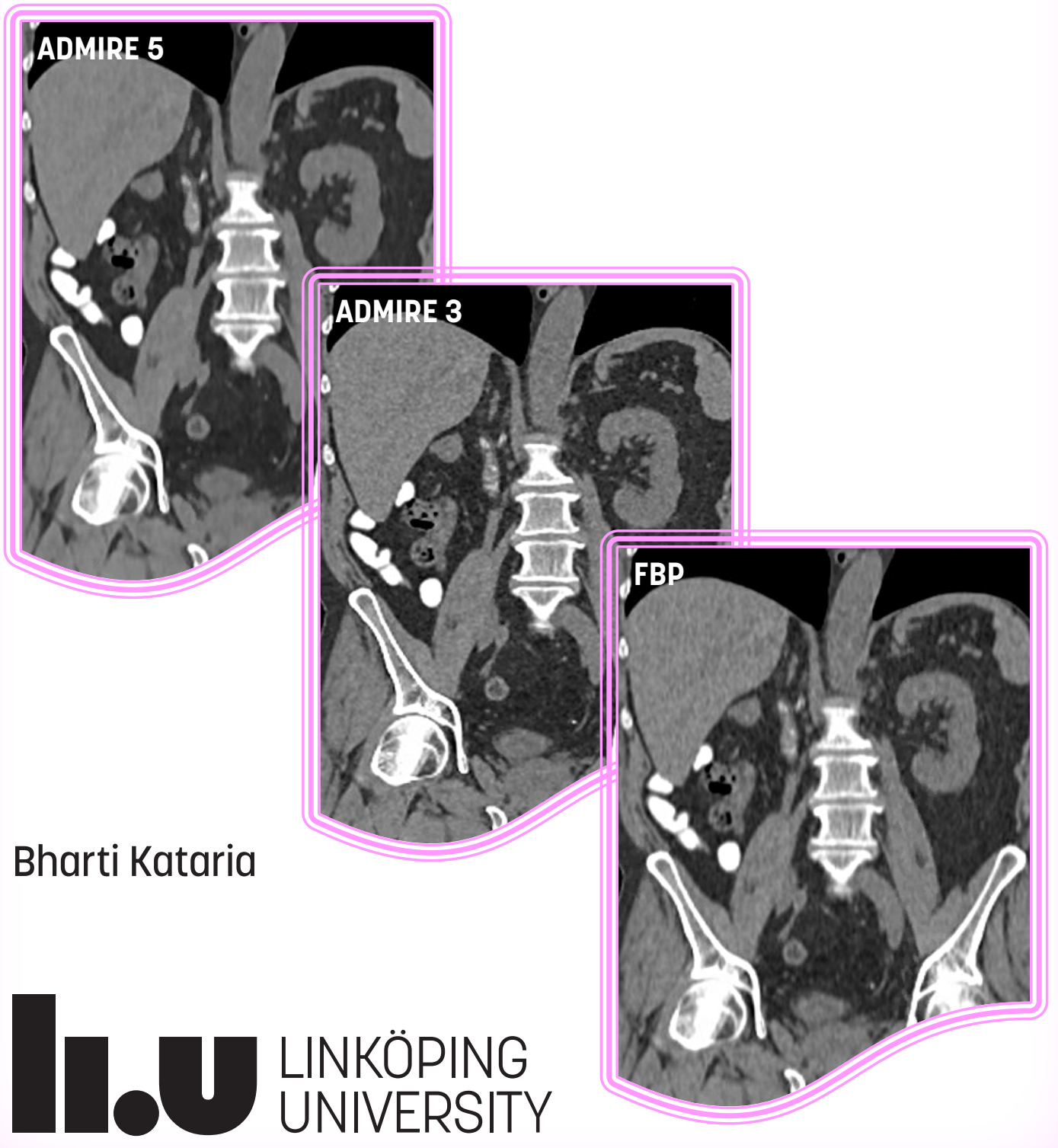


Linköping University Medical Dissertation No. 1683

\title{
Visual grading evaluation of reconstruction methods and dose optimisation in abdominal Computed Tomography
}

\author{
Bharti Kataria
}

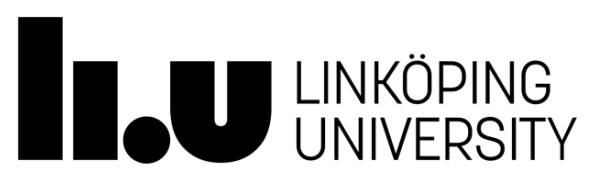

Department of Medical and Health Sciences

Linköping University, Sweden

Linköping 2019 
(CBharti Kataria, 2019

Cover images front: Demonstration of reconstruction algorithms used in abdominal CT

Cover image back: CT phantom; Catphan 504, Phantom Laboratory, Salem, NY

Design: Bharti Kataria

This work was conducted in collaboration with the Center for Medical Image Science and Visualisation (CMIV) at Linköping University, Sweden. CMIV is acknowledged for the provision and access to leading-edge research infrastructure.

Published articles have been reprinted with the permission of the copyright holder.

Printed in Sweden by LiU-Tryck, Linköping, Sweden, 2019

ISBN 978-91-7685-071-8

ISSN 0345-0082 
This thesis is dedicated to the loving memory of my dearest dad who inspired me to follow my dreams.

Progress is impossible without change, and those who cannot change their minds cannot change anything.

George Bernhard Shaw 1856-1950 



\section{Main Supervisor}

\section{Michael Sandborg, Professor}

Department of Medical Physics

Department of Medical \& Health Sciences

Linköping University

Linköping

Sweden

\section{Co-Supervisors}

Örjan Smedby, Professor

Department of Biomedical Engineering

\& Health Systems

KTH Royal Institute of Technology

Stockholm

Sweden

\author{
Anders Persson, Professor \\ Department of Radiology \\ Department of Medical \& Health Sciences \\ Center for Medical Image Science \& \\ Visualisation \\ Linköping University \\ Sweden
}

\author{
Hannibal Sökjer, Docent \\ Department of Radiology \\ Department of Medical \& Health Sciences \\ Linköping University \\ Linköping \\ Sweden
}

\section{Faculty opponent}

Håkan Geijer, Professor

Department of Radiology

School of Medical Sciences

Örebro University

Örebro

Sweden

\section{Faculty Board}

\section{Roger Siemund, Docent}

Department of Radiology

Department of Clinical Sciences

Lund University

Lund

Sweden

\section{Tomas Strömberg, Professor}

Department of Biomedical Engineering

Division of Biomedical Engineering

Linköping University

Linköping

Sweden

\section{Maria Engström, Professor}

Department of Medical Physics

Division of Radiological Sciences

Linköping University

Linköping

Sweden

\section{Substitute}

Eva Lund, Professor emerita

Department of Medical \& Health Sciences

Division of Radiological Sciences

Linköping University

Linköping

Sweden 



\section{CONTENTS}

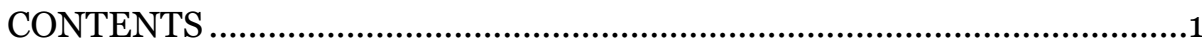

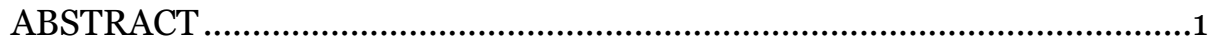

SVENSK SAMMANFATTNING ….......................................................... 3

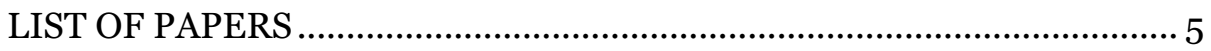

Peer-reviewed conference abstracts ........................................................ 7

AUTHOR CONTRIBUTIONS .................................................................

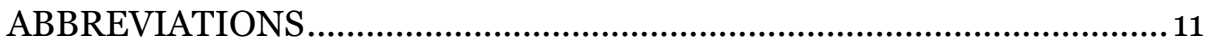

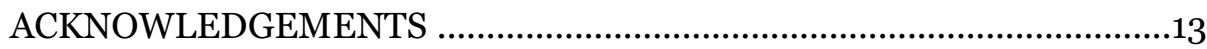

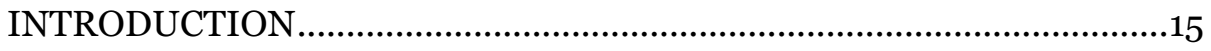

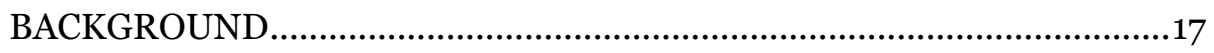

CT technique........................................................................................17

Radiation protection ……………....................................................... 18

Principles of radiation protection ........................................................ 18

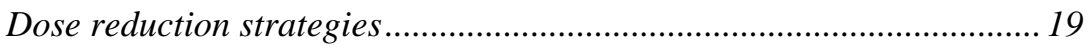

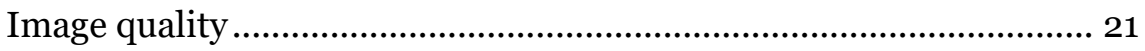

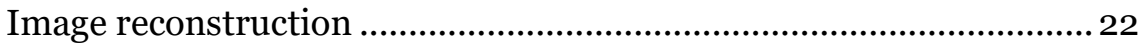

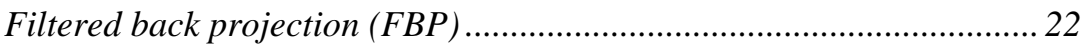

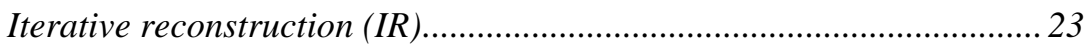

Image reformatting methods ................................................................. 28

Image quality evaluation ......................................................................28

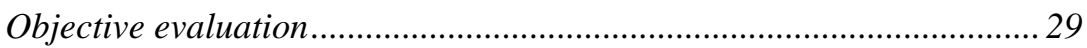

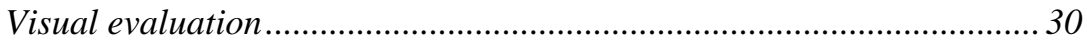

Radiographers' role in radiation protection .........................................40

AIMS............................................................................................... 43

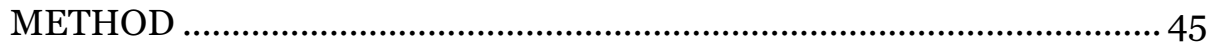

Research subjects .............................................................................. 45

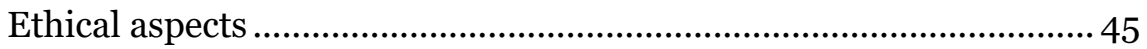

Inclusion and exclusion criteria ............................................................. 45

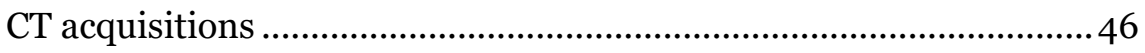




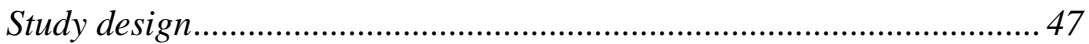

Assessment of image quality ..............................................................5

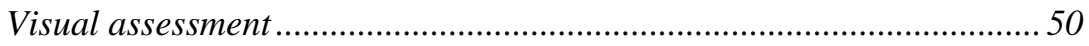

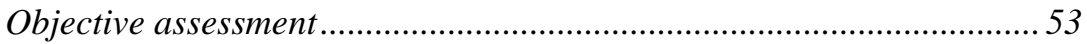

Statistical analysis ......................................................................... 54

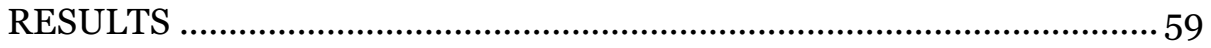

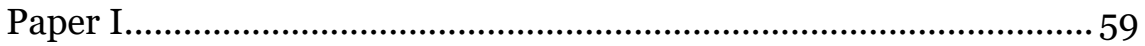

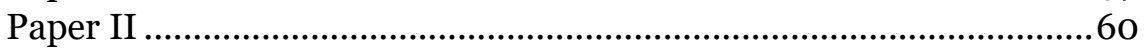

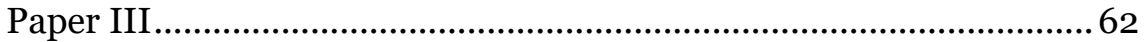

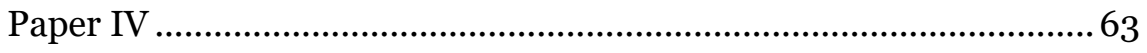

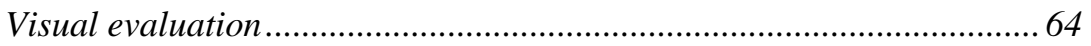

Objective evaluation........................................................................... 66

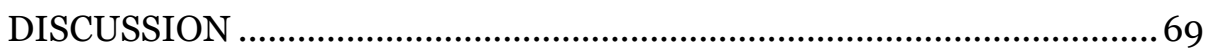

Radiation risks \& LNT........................................................................ 70

Method discussion............................................................................... 70

Statistical method ........................................................................... 72

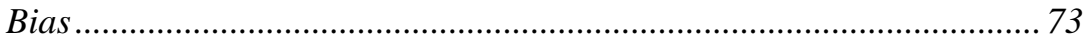

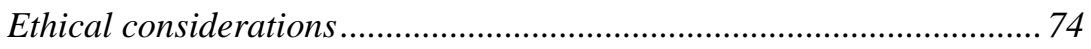

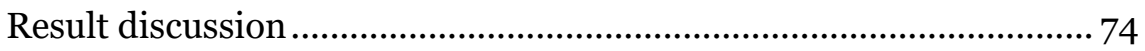

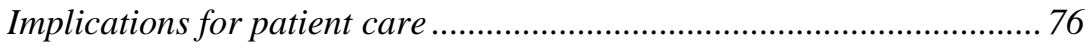

Radiographers' role ............................................................................ 76

Future aspects .................................................................................. 76

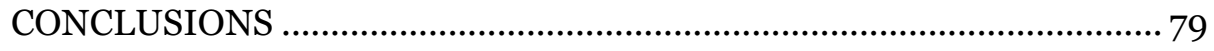

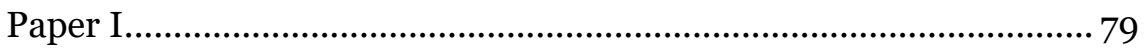

Paper II ...................................................................................... 79

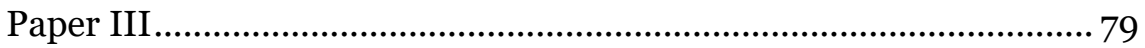

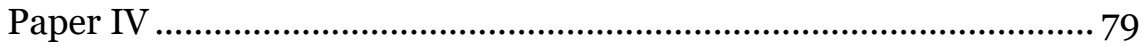

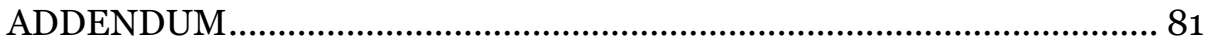

Other published papers not included in the thesis .............................. 81

Published paper related to but not part of the thesis .............................. 81

Published paper not related to the thesis ............................................... 81

Other peer reviewed conference abstracts............................................. 81

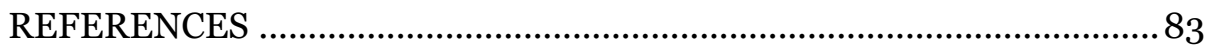




\section{ABSTRACT}

Since its introduction in the 1970's CT has emerged as a modality of choice because of its high sensitivity in producing accurate diagnostic images. A third of all Computed Tomography (CT) examinations are abdominal CTs which deliver one of the highest doses among common examinations. An increase in the number of CT examinations has raised concerns about the negative effects of ionising radiation as the dose is cumulative over the life span of the individual. Image quality in CT is closely related to the radiation dose, so that a certain dose with an associated small, but not negligible, risk is a prerequisite for high image quality. Typically, dose reduction in CT results in higher noise and a decrease in low contrast resolution which can be detrimental to the image quality produced. New technology presents a wide range of dose reduction strategies, the latest being iterative reconstruction (IR).

The aim of this thesis was to evaluate two different classes of iterative reconstruction algorithms: statistical (SAFIRE) and model-based (ADMIRE) as well as to explore the diagnostic value of a low-dose abdominal CT for optimisation purposes.

This thesis included a total of 140 human subjects in four image quality evaluation studies, three of which were prospective studies (Papers I, II and IV) and one retrospective study (Paper III). Visual grading experiments to determine the potential dose reductions, were performed with pairwise comparison of image quality in the same patient at different tube loads (dose) and reconstructed with Filtered back projection (FBP) and SAFIRE strength 1 in a low-dose abdominal CT (Paper I) and FBP and ADMIRE strengths 3 and 5 in a standard dose abdominal CT (Paper II). Paper IV evaluated the impact of slice thicknesses in CT images reconstructed with ADMIRE strengths 3 and 5 when comparing multiplanar reconstruction (MPR) formatted images in a standard dose abdominal CT. Paper III, on the other hand, was an absolute assessment of image quality and pathology between the three phases of a CT Urography (CTU) protocol to explore the diagnostic value of low-dose abdominal CT. The anonymised images were displayed in random order and image quality was assessed by a group of radiologists using image quality criteria from the "European guidelines of quality criteria for CT". The responses from the reviewer assessment were analysed statistically with ordinal logistic regression i.e. Visual Grading Regression (VGR).

Results in Paper I show that a small dose reduction (5-9 \%) was possible using SAFIRE strength 1 and indicated the need for further research to 
evaluate the dose reduction potential of higher strengths of the algorithm. In Paper II a 30\% dose reduction was possible without change in ADMIRE algorithm strength as no improvement in image quality was observed between tube loads 98- and 140 mAs. When comparing tube loads 42 and 98 mAs, further dose reduction was possible with ADMIRE strength 3 (2247\%). However, for images reconstructed with ADMIRE strength 5, a dose reduction of 34-74\% was possible for some, but not all image criteria. Image quality in low-contrast objects such as the liver parenchyma, was affected and a decline in diagnostic confidence was observed. Paper IV showed potential dose reductions are possible with increasing slice thickness from $1 \mathrm{~mm}$ to $2 \mathrm{~mm}$ (24-35\%) and $1 \mathrm{~mm}$ to $3 \mathrm{~mm}$ (25-41\%). ADMIRE strength 3 continued to provide diagnostically acceptable images with possible dose reductions for all image criteria assessed. Despite objective evaluations showing a decrease in noise and an increase in contrast to noise ratio, ADMIRE strength 5 had diverse effects on the five image criteria, depending on slice thickness and further dose reductions were limited to certain image criteria. The findings do not support a general recommendation to replace ADMIRE3 with ADMIRE5 in clinical abdominal CT protocols.

Paper III studied another aspect of optimisation and results show that visualisation of renal anatomy was as expected in favour of the post-contrast phases when compared to the native phase. Assessment of pathology showed no significant differences between the three phases. Significantly higher diagnostic certainty for renal anatomy was observed for the postcontrast phases when compared to the native phase. Significantly high certainty scores were also seen for the nephrographic phase for incidental findings. The conclusion is that a low-dose series seems to be sufficient as a first-line modality in certain patient groups.

This thesis clinically evaluated the effect of IR in abdominal CT imaging and estimated potential dose reductions. The important conclusion from papers I, II and IV is that IR improves image quality in abdominal CT allowing for some dose reductions. However, the clinical utility of the highest strength of the algorithm is limited to certain criteria. The results can be used to optimise the clinical abdominal CT protocol. The conclusion from paper III may increase clinical awareness of the value of the low-dose abdominal protocol when choosing an imaging method for certain patient groups who are more sensitive to radiation. 


\section{SVENSK SAMMANFATTNING}

Datortomografi (DT) används i allt större omfattning vid bilddiagnostik och ger en viss stråldos till patienten. DT är en viktig, snabb och patientvänlig undersökningsteknik. En fördel med denna teknik är att bildmaterialet kan rekonstrueras i olika format för att åskådliggöra anatomin på bästa sätt beroende på vilken frågeställning som ska besvaras. Joniserande strålning från dessa undersökningar anses öka risken för negativa effekter även om risken för den enskilde patient är mycket liten. Antalet datortomografiundersökningar ökar från år till år vilket kan leda till ökade stråldoser till befolkningen. Optimering av undersökningsteknik och val av undersökning för att minska negativa effekter av röntgenstrålning är därför nödvändig.

Det övergripande målet med avhandlingen var att utvärdera bildkvalitet vid en DT-undersökning av buken (då dessa medför en av de högsta stråldoserna bland de vanliga röntgenundersökningarna), att kvantifiera möjlig stråldosminskning med hjälp av iterativa rekonstruktionsalgoritmer och att utvärdera diagnostiska värdet av lågdosundersökningsteknik vid DT-buk. Av de fyra delstudierna var delarbeten I, II och IV prospektiva och delarbete III retrospektivt.

För de prospektiva studierna, samlades bildmaterial in vid en klinisk berättigad undersökning av lågdos-DT av buken (delarbetet I), eller standarddos-DT av buken (delarbetet II och IV). Bilder rekonstruerades med en standard bildrekonstruktionsalgoritm, filtrerad återprojektion (FBP), och med styrka 1 av den iterativa algoritmen SAFIRE (delarbetet I). I delarbeten II och IV, gjordes bildrekonstruktioner med FBP och med styrka 3 och 5 av den iterativa algoritmen ADMIRE. Avidentifierade bildmaterial för varje patient visades parvis i slumpmässig ordning för ett antal granskare och bildkvaliteten bedömdes med hjälp av europeiska bildkriterier. I den retrospektiva studien, delarbete III, hämtades bildmaterialet från utförda DT-urografiundersökningar från bildarkivet. För varje undersökning visades bilder från varje fas i DT-urografiundersökningen separat i slumpmässig ordning. För samtliga delarbeten, hämtades bildkriterierna från "European Guidelines of Quality Criteria for CT" och modifierades för att passa till varje studie. Granskarnas bedömning analyserades med ordinal logistisk regression så kallad visual grading regression (VGR).

Resultat från delarbetet I visade att det fanns en signifikant inverkan av dos ( $\mathrm{p}<0,001)$ och rekonstruktionsalgoritm $(\mathrm{p}<\mathrm{0,01})$ på samtliga bildkriterier, med en beräknad möjlig dosminskning på 5-9\%. 
Delarbetet II visade att rekonstruktionsalgoritmen ADMIRE förbättrar bildkvaliteten i jämförelse med FBP. ADMIRE styrka 3 tillåter en dosminskning mellan 22-47\% för samtliga bildkriterier medan ADMIRE styrka 5 tillăter en dosminskning mellan $34-74 \%$ för nästan alla bedömda bildkriterier utom återgivning av leverns parenkym. Ett mycket oväntat resultat var att bildkvalitén för 70\% dosnivå bedömdes som högre eller likvärdig med $100 \%$ dosnivå, vilket innebar att stråldosen kan sänkas med $30 \%$ utan förändring i algoritm eller styrka.

Resultaten av delarbete III visade att avbildning av njuranatomi var som förväntat för varje fas med fördel för kontrastuppladdningsfaserna jämfört med den nativa fasen. Detta var inte ett oväntat resultat eftersom DT-urografiprotokollet är utformat för att visualisera njuranatomi på bästa möjliga sätt. Vid bedömning av patologiska fynd, erhölls dock små och icke signifikanta skillnader mellan faserna. Däremot noterades signifikant högre bedömningssäkerhet för patologi i njurarna för de kontrast förstärkta faserna jämfört med nativfasen, och endast för bifynd signifikant högre poäng för parenkymfasen.

Delarbete IV visade att styrka 5 jämfört med styrka 3 av den iterativa rekonstruktionsalgoritmen, har olika effekter på bedömningen av bildkvalitetskriterierna. Ökning av MPR-snittjocklek från $1 \mathrm{~mm}$ till $2 \mathrm{~mm}$ eller 3 $\mathrm{mm}$, ger en förbättring i bildkvalité, vilket möjliggör en viss dosreduktion. Den kliniska användbarheten av ADMIRE styrka 5 är begränsad, medan ADMIRE styrka 3 levererar bättre bildkvalitet för samtliga undersökta bildkriterier vid datortomografiundersökning av buken.

Den viktigaste slutsatsen av delarbeten I, II och IV är att iterativa rekonstruktionsalgoritmer förbättrar bildkvalitet jämfört med FBP för samma stråldos och en dosminskning är möjlig. Detta kan användas för att optimera det kliniska DT-bukundersöknings protokoll. Slutsatsen för delarbetet III var att en lågdos-DT-bukundersökning är ett av många dosreduceringsalternativ, som möjligen kan användas för att minska strålningsbördan hos vissa patientgrupper som är mer känsliga för röntgenstrålning. 


\section{LIST OF PAPERS}

This thesis is based on the following papers, referred to in the text by their roman numerals (I-IV).

I. Patient dose and image quality in low-dose abdominal CT: a comparison between iterative reconstruction and Filtered back projection.

Kataria B and Smedby Ö

Acta Radiologica 2013;54: 540-548

II. Assessment of image quality in abdominal CT: potential dose reduction with model-based iterative reconstruction. Kataria B, Nilsson Althén J, Smedby Ö, Persson A, Sökjer H and Sandborg M

European Radiology 2018;28:2464-2473

III. Image quality and pathology assessment in CT Urography: When is a low-dose series sufficient?

Kataria B, Nilsson Althén J, Smedby Ö, Persson A, Sökjer H and Sandborg M

BMC Medical Imaging 2019,19:64

IV. Assessment of image quality in abdominal CT: Effect of model-based iterative reconstruction, multi-planar reconstruction and slice thickness on potential dose reduction.

Kataria B, Nilsson Althén J, Smedby Ö, Persson A, Sökjer H and Sandborg M

Submitted for journal publication, 2019 
Visual grading evaluation and optimisation in abdominal Computed Tomography 


\section{Peer-reviewed conference abstracts}

- Patient dose and image quality in low-dose abdominal Computed Tomography: a comparison between iterative reconstruction and Filtered back projection.

Kataria B and Smedby Ö

European Congress of Radiology (ECR) Vienna, Austria 2013; Poster C-0547

- A comparison of patient dose and image quality in lowdose abdominal Computed Tomography (CT) between iterative reconstruction and Filtered back projection.

Kataria B and Smedby Ö

International Society of Radiographers and Radiological

Technicians (ISRRT) Seoul, Korea 2016; Oral presentation AFoo28

- Potential dose reduction in abdominal CT using a modelbased iterative reconstruction.

Kataria B, Nilsson Althén J, Smedby Ö, Persson A, Sökjer H and Sandborg M

European Congress of Radiology (ECR) Vienna, Austria 2017; Poster B-0845

- Potential dose reduction in abdominal CT using a modelbased iterative reconstruction.

Kataria B, Nilsson Althén J, Smedby Ö, Persson A, Sökjer H and Sandborg M

European Congress of Radiology (ECR) Vienna, Austria 2017; Oral presentation $\mathrm{SS} 1005$

- Optimisation in abdominal CT: a comparison between Filtered back projection and model-based iterative reconstruction.

Kataria B, Nilsson Althén J, Smedby Ö, Persson A, Sökjer H and Sandborg M

International Society of Radiographers and Radiological Technicians (ISRRT) Port of Spain, Trinidad \& Tobago 2018; Oral presentation CT 5-1

- Effect of tube load, model-based iterative reconstruction (MBIR) and slice thickness in abdominal CT using multiplanar reconstruction (MPR).

Kataria B, Nilsson Althén J, Smedby Ö, Persson A, Sökjer H and Sandborg M

European Congress of Radiology (ECR) Vienna, Austria 2019; Poster $\mathrm{C}-1185$ 
Visual grading evaluation and optimisation in abdominal Computed Tomography 


\section{AUTHOR CONTRIBUTIONS}

\section{Paper I}

Patient dose and image quality in low-dose abdominal CT: a comparison between iterative reconstruction and Filtered back projection.

Kataria B and Smedby Ö, Acta Radiologica 2013;54:540-548

\begin{tabular}{|l|c|c|}
\hline \multirow{2}{*}{ Project } & \multicolumn{2}{|c|}{$\begin{array}{c}\text { Author } \\
\text { contribution }\end{array}$} \\
\cline { 2 - 3 } & BK & ÖS \\
\hline Study conception and design & S & L \\
\hline Data acquisition & L & S \\
\hline Statistical analysis & S & L \\
\hline Data interpretation & S & L \\
\hline Funding acquisition & - & L \\
\hline Supervision & - & L \\
\hline Manuscript & \multicolumn{2}{|c|}{} \\
\hline Writing-original draft & L & S \\
\hline Journal correspondence & L & - \\
\hline Writing-review/editing & L & S \\
\hline
\end{tabular}

$\mathrm{S}=$ supporting, $\mathrm{L}=$ lead, BK= Bharti Kataria, ÖS= Örjan Smedby

\section{Paper II}

Assessment of image quality in abdominal CT: potential dose reduction with model-based iterative reconstruction.

Kataria B, Nilsson Althén J, Smedby Ö, Persson A, Sökjer H, Sandborg M, European Radiology 2018;28:2464-2473

\begin{tabular}{|l|c|c|c|c|c|c|}
\hline \multirow{2}{*}{ Project } & \multicolumn{6}{|c|}{ Author contribution } \\
\cline { 2 - 8 } & BK & JN & ÖS & AP & HS & MS \\
\hline Study conception and design & E & S & E & S & S & E \\
\hline Data acquisition & L & S & - & - & - & S \\
\hline Statistical analysis & S & - & L & - & - & S \\
\hline Objective data analysis & S & S & - & - & - & L \\
\hline Data interpretation & E & S & E & S & S & E \\
\hline Funding acquisition & S & - & - & - & - & L \\
\hline Supervision & - & S & S & S & S & L \\
\hline Manuscript & \multicolumn{7}{|l|}{} \\
\hline Writing-original draft & L & S & S & S & S & S \\
\hline Journal correspondence & L & - & - & - & - & - \\
\hline Writing-review/editing & L & S & S & S & S & S \\
\hline
\end{tabular}

$\mathrm{S}=$ supporting, $\mathrm{L}=$ lead, $\mathrm{E}=$ equal

$\mathrm{BK}=$ Bharti Kataria, $\mathrm{JN}=$ Jonas Nilsson Althén, $\mathrm{O} \mathrm{S}=$ Örjan Smedby, AP= Anders Persson, HS= Hannibal Sökjer, MS= Michael Sandborg 


\section{Paper III}

Image quality and pathology assessment in CT Urography: When is a low-dose series sufficient?

Kataria B, Nilsson Althén J, Smedby Ö, Persson A, Sökjer H, Sandborg M, BMC Medical Imaging 2019;19:64

\begin{tabular}{|l|c|c|c|c|c|c|}
\hline \multirow{2}{*}{ Project } & \multicolumn{7}{|c|}{ Author contribution } \\
\cline { 2 - 8 } & BK & JN & ÖS & AP & HS & MS \\
\hline Study conception and design & E & S & E & S & S & S \\
\hline Data acquisition & L & S & - & - & - & S \\
\hline Statistical analysis & S & - & L & - & - & S \\
\hline Data interpretation & E & S & E & S & S & E \\
\hline Funding acquisition & S & - & - & - & - & L \\
\hline Supervision & - & S & S & S & S & L \\
\hline Manuscript & \multicolumn{7}{|c|}{} \\
\hline Writing-original draft & L & S & S & S & S & S \\
\hline Journal correspondence & L & - & - & - & - & - \\
\hline Writing-review/editing & L & S & S & S & S & S \\
\hline
\end{tabular}

$\mathrm{S}=$ supporting, $\mathrm{L}=$ lead, $\mathrm{E}=$ equal

$\mathrm{BK}=$ Bharti Kataria, JN= Jonas Nilsson Althén, ÖS= Örjan Smedby, AP= Anders Persson, HS= Hannibal Sökjer, MS = Michael Sandborg

\section{Paper IV}

Assessment of image quality in abdominal CT: Effect of a combination of slice thickness and a model-based iterative reconstruction on potential dose reduction.

Kataria B, Nilsson Althén J, Smedby Ö, Persson A, Sökjer H, Sandborg M. Submitted for journal publication, 2019

\begin{tabular}{|l|c|c|c|c|c|c|}
\hline \multirow{2}{*}{ Project } & \multicolumn{6}{|c|}{ Author contribution } \\
\cline { 2 - 8 } & BK & JN & ÖS & AP & HS & MS \\
\hline Study conception and design & E & S & E & S & S & E \\
\hline Data acquisition & E & S & - & - & - & E \\
\hline Statistical analysis & S & - & L & - & - & S \\
\hline Objective data analysis & S & S & - & - & - & L \\
\hline Data interpretation & E & S & E & S & S & E \\
\hline Funding acquisition & S & - & - & - & - & L \\
\hline Supervision & - & S & S & S & S & L \\
\hline Manuscript & \multicolumn{7}{|c|}{} \\
\hline Writing-original draft & L & S & S & S & S & S \\
\hline Journal correspondence & L & - & - & - & - & - \\
\hline Writing-review/editing & L & S & S & S & S & S \\
\hline
\end{tabular}

$\mathrm{S}=$ supporting, $\mathrm{L}=$ lead, $\mathrm{E}=$ equal

$\mathrm{BK}=$ Bharti Kataria, JN= Jonas Nilsson Althén, ÖS= Örjan Smedby, AP= Anders Persson, HS= Hannibal Sökjer, MS= Michael Sandborg 


\section{ABBREVIATIONS}

ADMIRE Advanced modeled iterative reconstruction

AEC Automatic exposure control

AHARA As high as reasonably achievable

ALARA As low as reasonably achievable

ANOVA Analysis of variance

ART Algebraic iterative reconstruction technique

ATCM Automatic tube current modulation

AUC Area under the curve

BMI Body mass index

BSS Basic safety standards

CME Continuing medical education

CNR Contrast-to-noise ratio

CCTA Cardiac computed tomography angiography

CPD Continuing personal development

CTA Computed tomography angiography

CT Computed tomography

CTDI $_{\text {vol }} \quad$ Volume computed tomography dose index

CTU CT Urography

DLP Dose length product

DRL Diagnostic reference level

DSCT Dual-source computed tomography

FBP Filtered back projection

FPF False positive fraction

GLLAM Generalised linear and latent mixed models

HU Hounsfield units

IC Image criteria

ICRP International commission for radiation protection

ICS Image criteria scoring

IR Iterative reconstruction 


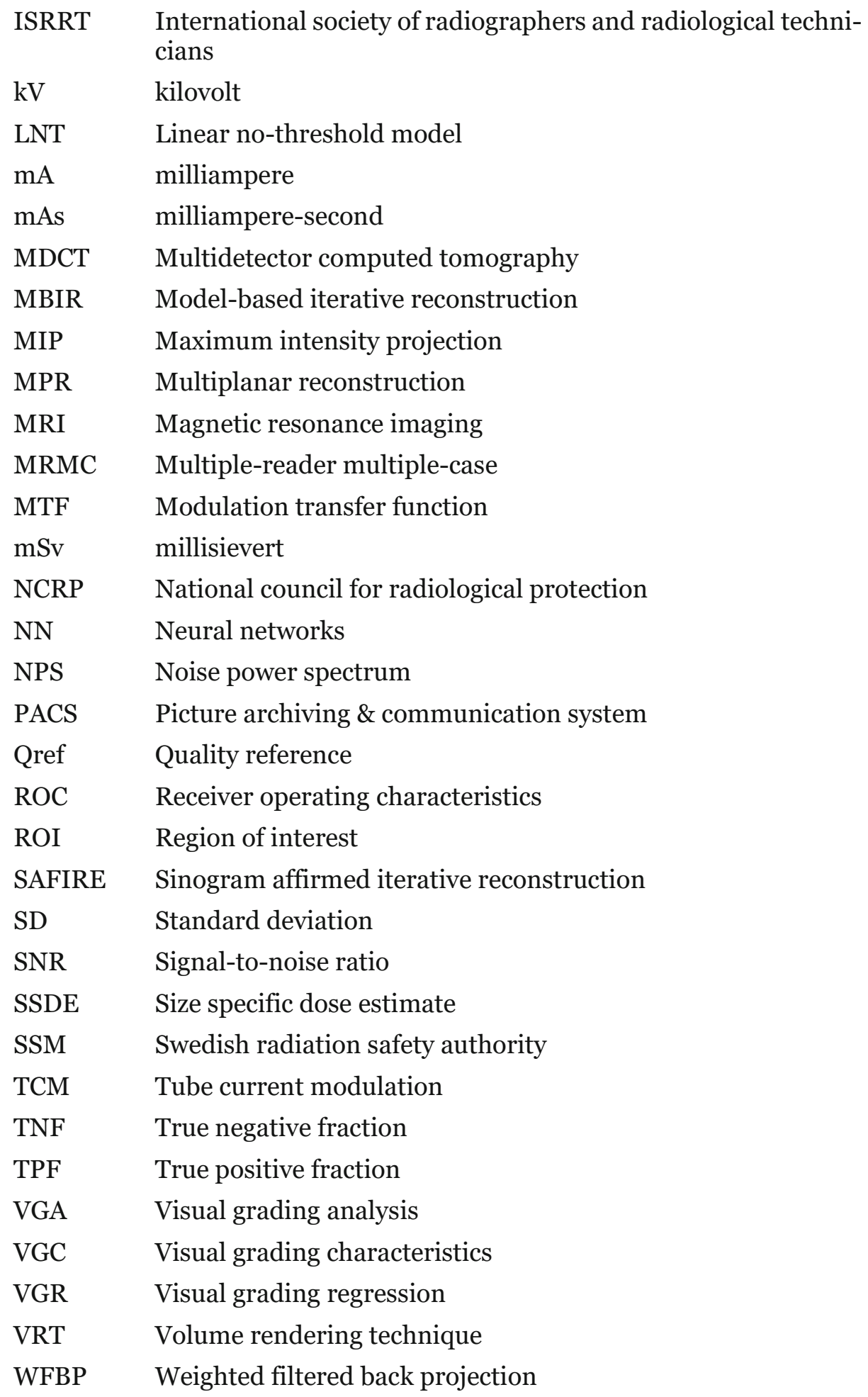




\section{ACKNOWLEDGEMENTS}

This exploration of the academic world has been a remarkable journey with many lessons learnt. I have encountered so many people who have encouraged and contributed towards the successful completion of my thesis. I take this opportunity to express my gratitude to all who helped accomplish this task.

Firstly, I extend my thanks to my main supervisor Michael Sandborg for prompt constructive feedback, help with the objective tests and taking time to patiently explain the intricacies of the physics of image quality assessments. I am also thankful to my former main supervisor and now cosupervisor, Örjan Smedby for paving my way to a PhD. It was encouraging to see your enthusiasm and knowledge in statistical analysis and how you enlightened me about the complexities of logistic regression analysis. Thank you both for believing in me and pushing me out of my comfort zone to meet the challenges of research and writing whenever I hesitated. My co-supervisors Anders Persson and Hannibal Sökjer, it has been an honour to work with you. Thank you both for your support and the informal encouragement chats we had whenever we got together over a cup of coffee or lunch that helped to guide me on my journey. Jonas Nilsson Althén, I thank you for your support and guidance throughout my academic studies and providing me with ideas on how to further my research. Some thanks are also due to Mannudeep Kalra who visited us briefly at CMIV and provided me with useful tips on how to plan our research project. Staffan Wirell is also acknowledged for his knowledge and guidance at my first attempt at research and academic writing of my bachelor's thesis. Your encouragement helped me to embark on this academic journey. You are all excellent peers; in whose footsteps I would gladly like to follow.

My sincere gratitude goes to all my readers: Anki Pozson, Bo Ekerling, Jenny Öman, Johan Asplund, Lasse Pettersson, Peter Johansson, Senija Halilic and Thomas Wiessler for your excellent performance in grading of the images despite the time pressure and tedious nature of the assignment. Without you all, there would not have been any results to analyse.

I owe my deepest gratitude to Håkan Gustafsson who supported my projects when I struggled to find ways to avoid delays in the grading of images due to financial difficulties.

I am grateful to my father (in memoriam) and my mother for providing a solid education foundation from the best institutions and supporting me financially during my training as a radiographer in England even though 
times were hard. I miss you dad and am sorry you are not with us today to share in my moment of pride.

I would also like to thank my champion, mentor and cousin Ramesh Raja for guiding me into a career in radiography. Without you I would not be standing here today.

I am indebted to my informal "language checkers" Chrissy and Chan Kataria, Aarthi Ramlaul and my former Bristol student colleague and best friend Sue Douglas, who as native English speakers, helped me proofread my papers before submission. Thanks, are also due to all colleagues who helped to proofread my thesis before publication.

I express my gratitude to my many colleagues both new and old, who have held the fort in the radiology department while I was engaged in academia and to the management Mathias Axelsson, Anna Köpberg and Susanne Hellberg Karlsson for providing the opportunity to combine work with PhD studies.

Special thanks to the PhD students and staff at CMIV, for providing the research facilities and academic environment, particularly Petter Quick for help in the construction of the examination protocols on the CT and guidance in understanding the image reconstruction and archiving process. Thanks to my colleague Lina Djupman who gave up her free time to assist me in examining all the patients included in papers II and IV.

Thanks even to my former colleagues at Vrinnevi Hospital who helped to recruit patients for paper I, without your help, it would have been a slow process to collect all the data.

My colleagues and close friends Lise-lotte Lundvall and Eva Hellman for the many discussions that have helped me to refocus and improve my writing skills in Swedish. And my dearest friend and study colleague Birgitta Wennberg for the interesting discussions and for putting everything into the right perspective. Thank you for being there for me through all the emotional and stressful times in my life.

My conference colleague Aarthi Ramlaul for your encouragement, support and companionship during conference attendances. Your friendship and professional aptitude have contributed positively to my intellectual development. Your support during the past year has meant a lot to me.

Finally, I extend my special thanks to my family, my husband Vin and my two beautiful daughters Krish and Kirna who have lovingly supported me through the years. Without you all, this accomplishment would not have been possible. 


\section{INTRODUCTION}

CT has emerged as an invaluable tool in diagnostic imaging, offering an expanding variety of cross-sectional imaging applications at high specificity and sensitivity [1]. It provides volume data that can be acquired without superimposition of anatomical details. Since its introduction in 1972, the utility of Computed Tomography (CT) in medical imaging has increased due to evolving technological advancements from single slice to spiral/helical (1990) and multidetector (MDCT 1998). Faster scan times facilitate quick diagnostics leading to economic benefits in health care due to improvements in workflow [2]. However, the use of CT comes at a cost, the most important is radiation dose resulting from CT examinations.

There has been a two-fold increase in the number of examinations performed globally over the past decade with a steady growth rate of $\approx 10 \%$ per year, thereby increasing the absorbed dose from ionising radiation to the general population. Concerns have been raised as to the adverse effects of ionising radiation in the range of doses delivered by CT [3]. CT constitutes approximately $15 \%$ of all medical imaging procedures that use ionising radiation, but delivers $75 \%$ of the total effective dose $[4,5]$. Thirty percent of all CT examinations are in the abdominal/pelvic region and multiphase as well as follow-up examinations in the abdomen are quite common. Multiple scans thus further increase the absorbed dose and lifetime risk of cancer for the individual $[1,2,6]$.

Published papers have suggested that there may be a small but significant increase in risk of radiation-induced cancers from exposure to radiation from CT. These claims are based on extrapolation of dose and radiation risk data from the follow-up after the nuclear bombs in Hiroshima and Nagasaki. The Linear no-threshold model (LNT) stems from estimating cancer risk on computer and epidemiological models [7, 8, 9, 10, 11]. However, the validity of the LNT has been questioned in reports that argue that low doses of ionising radiation are beneficial $[12,13,14]$. Prospective data from a recent study has highlighted a linear relationship between irradiation of the brain and risk of developing a tumour in the brain. Similarly, an irradiation of bone marrow may lead to development of leukaemia in children [15]. Mathews et al [16] validated these findings showing a $24 \%$ increase in risk of developing cancer in a paediatric population cohort who were exposed to a CT scan one year prior to the cancer diagnosis. While these studies clarified how radiation dose affects the paediatric population, there is no evidence to suggest how this effect would transfer on to the adult population. However, the increasing number of $\mathrm{CT}$ examinations provide 
reasons for concern especially in the paediatric population where decrease in scan times have rendered a significant increase in the number of CT examinations in children, as the need for sedation is reduced [17]. Even if the individual risk in the adult population is very small and perhaps difficult to prove, the increase in collective population dose may give rise to a public health problem if this individual small risk is applied to a large number of individuals [18].

Given the fact that the use of CT is mostly beneficial, when the benefit outweighs the risk and the investigation is justified to improve patient outcome, gentle and wise use of this technique is advocated. However, reports from various institutions suggest that $20 \%$ up to $50 \%$ of investigations performed are not appropriate $[19,20]$. The most common reasons being that the referrals do not meet the appropriateness criteria or that they lack relevant clinical information [19, 20, 21]. It is apparent that radiation protection principles should always be applied and that care should be taken in daily radiological practice when determining the appropriateness of the referrals and their clinical significance in justification of the examination $[1$, $6,7,20]$. Optimisation of clinical protocols is advocated in accordance with best practice and patient safety regulations, i.e. radiation dose for every examination should be as low as reasonably achievable (ALARA principle) while maintaining good image quality according to the AHARA principle (as high as reasonably achievable) [22].

Image quality in CT is closely related to the absorbed dose and noise, where a certain dose is required for sufficient image quality. Any dose reduction results in an increase in image noise and artefacts and may thus diminish image quality. Modern CT equipment uses techniques for dose reduction such as filters, dynamic collimation, automatic exposure control (AEC) systems, dose efficient detectors and advanced software solutions such as iterative reconstruction algorithms [1].

Maintaining image quality with reduction in dose is a major challenge especially for tasks which require higher low-contrast resolution such as the abdominal organs. Iterative reconstruction algorithms suppress noise thus improving image quality and possibly allow for potential dose reductions. The main objective of this thesis is to evaluate several aspects of image quality using iterative reconstruction and estimate dose reduction potential whilst maintaining the clinically acceptable image quality in abdominal CT. The implementation of low-dose abdominal CT has been generally very slow, hence it was of interest to study the diagnostic value of low-dose abdominal CT. 


\section{BACKGROUND}

\section{CT technique}

The slip-ring technology, high-power x-rays tubes and advanced interpolation algorithms were instrumental in the advent of multi-detector Computed Tomography (MDCT) in the 1990's [1]. Advances in CT hardware during the past 2 decades have increased the spectrum of applications available for this modality today. An exponential increase in the number of slices, with a doubling of slices every 2.5 years, led to faster scan speeds, an increase in transverse resolution and an alteration in the shape of the x-ray beam from fan (4-8 slices) to cone-beam (16-64 slices) [23]. The requirement for temporal resolution in CT angiography (CTA), Cardiac CT angiography (CCTA), perfusion and dynamic scan applications was met with the evolvement of large areas detectors (128, 256 and 320 slice MDCT systems) and 192 x 2 slice dual-source CT (DSCT) [1].

MDCT facilitated contiguous data acquisition of a whole volume incorporating 3-dimensional imaging with near isotropic resolution. Isotropic resolution also permitted the development of three-dimensional post-processing techniques such as multi-planar reconstruction (MPR), maximum intensity projection (MIP) and volume-rendering techniques (VRT) [22, 24].

In MDCT, data acquisition is performed with simultaneous rotation of the $\mathrm{x}$-ray tube and detector-array around the patient synchronised with a linear table feed resulting in a helical scan. Axial scanning technique is also possible. As the $\mathrm{x}$-ray beam passes through the patient, some radiation is absorbed whereby the attenuation profile projected onto the detector elements is measured. These measured linear attenuation coefficient values $(\mu)$ in each voxel of the image matrix are represented as grey levels and transformed into CT-values (also known as Hounsfield units, HU), using the equation below. The CT value of o $\mathrm{HU}$ is assigned to water and CT value of $-1000 H U$ to air on this scale [25].

$$
\text { CT value }=\frac{\mu_{\text {voxel }}-\mu_{\text {water }}}{\mu_{\text {water }}} \times 1000
$$

A DSCT system consists of an assembly of two x-ray tubes and corresponding detectors mounted at a $90^{\circ}$ angular off-set. One of the detectors covers the whole field of view while the other is restricted to the smaller central field of view $[22,24,26]$. When both x-ray sources use the same 
tube voltage, high speed scanning with flash technique for faster coverage of the area of interest is achieved. When different tube voltages are applied to each of the x-ray sources, two dual-energy (DECT) data sets obtained simultaneously in one scan can be used to differentiate, characterise, isolate and distinguish the imaged materials and tissues [27].

\section{Radiation protection}

Absorbed doses per examination in CT have decreased over the past two decades due to technological advancements, although scans are performed with thinner slice thicknesses to improve spatial resolution [28]. When using CT in clinical practice, it is important to recognise the relationship between image quality and absorbed dose overall and to follow the radiation protection principles as the clinical benefit of CT in most cases far outweighs the risks associated with such radiation exposure. There are robust mechanisms involved in the regulation of the use of ionising radiation and radiation protection in medical care. Both national and international institutions such as the Swedish Radiation Safety Authority (SSM) [29], the International Commission for Radiation Protection (ICRP) [30] and International Atomic Energy Agency (IAEA) [31] provide guidance in maintaining the balance between risks and benefits and issue recommendations as to the use of the radiation protection principles in clinical practice.

There are essentially two types of adverse health effects involved with exposure to ionising radiation; deterministic and stochastic effects. The fundamental purpose of radiation protection principles is to protect human health by preventing deterministic effects from arising and to reduce the risk of stochastic effects to as low as reasonably achievable [30]. The risk for deterministic injury occurring is higher for interventional procedures with higher skin dose from a single procedure compared to CT. However, the risk for deterministic effects cannot be ruled out if multiple or repeated high dose procedures such as perfusion studies and angiographies are performed. When performed on the same patient over the same anatomical region, temporary hair loss in a band shape has been reported [3]. Stochastic effects are cancer induction and genetic effects. Even though the probability of these effects occurring is very small at doses lower than $100 \mathrm{mSv}$, the LNT model is still considered the best practical approach in managing risks from radiation exposure, according to the ICRP, until new evidence provides an alternative model [30, 32, 33, 34].

\section{Principles of radiation protection}

To protect the patients from the detrimental effects of ionising radiation, the three principles deployed are justification, optimisation and diagnostic reference levels [30]: 
- Justification is a powerful radiation protection tool in the prevention of unnecessary exposure to ionising radiation. The profession is responsible for the justification of the procedure of choice and should have adequate special training in radiation protection to justify that the benefit of the procedure always outweighs the detrimental risk of the associated dose and other examination associated risks.

- Optimisation is a process where the absorbed dose to the irradiated subject during a radiological procedure is kept as low as reasonably achievable (ALARA) without compromising the desired image quality required for diagnostic purposes and is applicable to situations that are justified. Hence the image quality should be as high as reasonably achievable (AHARA). Good radiation protection practice involves a balance between the two essences of the optimisation process; ALARA and AHARA [22]. Dose optimisation can be also achieved by limiting the number of CT scans and the collective effective dose especially in young adults and children who are more sensitive to radiation and have a longer life expectancy. An increase in scan length and number of phases performed both tend to increase the dose. Hence, limiting the scan length to the region of interest (ROI) and number of phases to the indications on the referral, are measures that can be taken for radiation protection.

- Diagnostic Reference Levels (DRL) are "trigger levels" used to identify unnecessarily high dose procedures in order to initiate optimisation and dose reduction, without compromising the required diagnostic image quality. DRLs can be defined at local, national or international levels, the upper limits of DRLs are derived by calculating the $75^{\text {th }}$ percentile of observed doses for a particular examination. The Swedish radiation safety authority (SSM) has developed a web based tool "Dosreg" (https://dosreg.ssm.se/) to assist healthcare providers with a tool to optimise radiological examinations. The main purpose of the database is to streamline the process from collection to analysis in order to determine new or revised reference levels. The interface also facilitates data collection for estimating the radiation dose to the population.

To protect medical staff from radiation, the principle of dose limitation is applied.

\section{Dose reduction strategies}

Evolving CT technique allows for dose reduction whilst maintaining the image quality required for diagnostic purposes. Recent technological advancements have improved the dose-efficiency of modern CT systems. A range of dose reduction features are readily available in clinical practices and include automatic tube current modulation (ATCM), automatic 
kilovoltage $(\mathrm{kV})$ selection, flying focus technology, iterative reconstruction algorithms, high-power x-ray tubes and dose efficient detectors, among others. These emerging techniques are used to achieve the desired image quality with reduced dose when optimal imaging protocols are developed.

\section{Automatic tube current modulation (ATCM)}

ATCM was introduced to compensate for the differences in patient attenuation and to ensure a more homogenous image quality with reductions in the tube current time product (mAs) typically between $10 \%$ and $50 \%$, without detriment to image quality. ATCM enabled more efficient use of the available $\mathrm{x}$-ray power by adopting the tube current $(\mathrm{mA})$ to the patient attenuation profile as a function of the projection angle [22]. The CT system determines the beam attenuation of the individual patient from a localiser radiograph and tailors the scanner output to the specific patient body habitus.

Tube Current Modulation (TCM) techniques are based on either reference image quality or estimated image noise (noise index) and are vendor dependent. For optimal function of the (TCM), patient centring at isocentre in the scan field of view is critical as patient attenuation is estimated from the localiser radiograph.

The two components of TCM are angular modulation and longitudinal modulation (Figure 1). Angular-TCM compensates for changes in attenuation profile in the $\mathrm{x}-\mathrm{y}$ plane by modulating the $\mathrm{mA}$ with the primary aim of reducing dose. The longitudinal-TCM modulates the $\mathrm{mA}$ in the z-direction by regulating the tube current to patient attenuation profile changes with table feed, thus maintaining a constant level in image quality throughout the scan.

\section{Automatic kilovoltage (kV) selection}

Automatic $\mathrm{kV}$ selection is a task-based tool that has recently been introduced in clinical practice as a dose saving strategy. With a lower $\mathrm{kV}$, an increase in noise is observed as energy penetration decreases. However, the lower $\mathrm{kV}$ also increases image contrast and $\mathrm{CNR}$, both of which improve image quality and help to reduce radiation dose. Depending on the body region, the diagnostic task at hand and patient attenuation characteristics, the selection of appropriate $\mathrm{kV}$ setting will automatically optimise the tube voltage simultaneously as the tube current is adjusted by the TCM to maintain a constant contrast-to-noise (CNR) ratio [35]. 

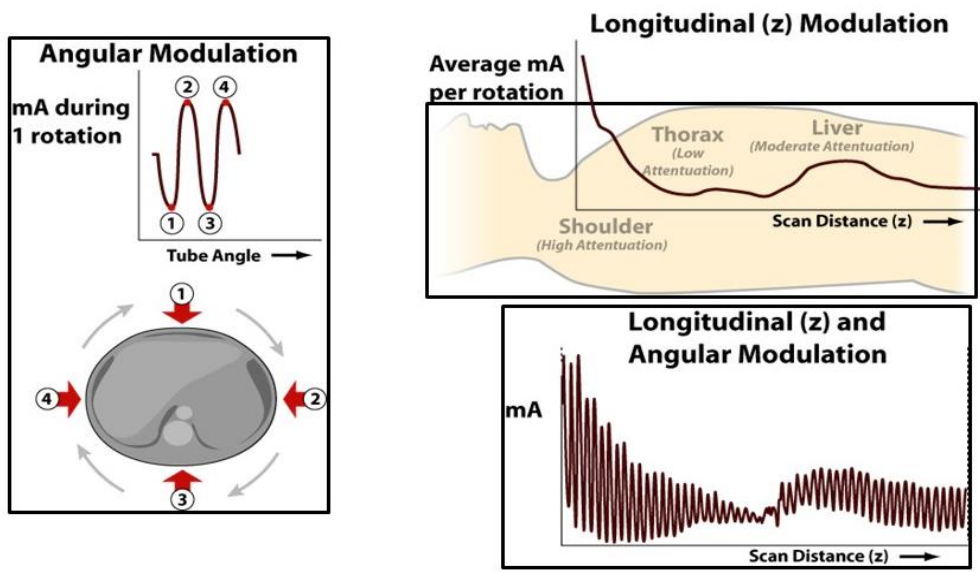

Figure 1. Angular \& Longitudinal tube current modulation according to patient attenuation characteristics [36]. Retrieved 15 February, 2015 from http://www.aapm.org/pubs/CTProtocols/documents/EducationSlides .pptx Reproduced with permission from AAPM Working Group on CT Nomenclature and Protocols, AAPM Computed Tomography Radiation dose education slides.

\section{Flying focus technology}

An increase in sampling frequency was achieved by electronically steering quick changes in focal spot position controlled by an electromagnetic field in the trans-axial plane. Although an increase in sampling frequency is often associated with dose increase, the flying focus technology is exempted from this penalty. Target switching of focus in $x-y$ plane improves spatial resolution, whereas the double z-plane sampling deals with windmill artefacts and improvement in longitudinal resolution [37]. This technology is used by one vendor to double the number of slices e.g. from 64 to 128 to enable faster scan times and improve longitudinal resolution [22].

\section{Image quality}

Image quality in CT is closely related to the absorbed dose as a certain dose is a prerequisite for sufficient image quality. There are many factors that influence image quality and absorbed dose in CT. If all other parameters remain constant, the effects of some of the important parameters are outlined in Table 1. 
Table 1. Effect on absorbed dose and image quality with variation in Computed Tomography parameters, if all other parameters remain constant. The arrows explain the relationship between the scan parameters, absorbed dose, and subsequently image quality.

\begin{tabular}{|c|c|c|}
\hline $\begin{array}{c}\text { Scan } \\
\text { parameters }\end{array}$ & $\begin{array}{l}\text { Effect on ab- } \\
\text { sorbed dose }\end{array}$ & $\begin{array}{l}\text { Effect on image quality } \\
\text { (IQ) }\end{array}$ \\
\hline Tube current (mA) & 1 with increased $\mathrm{mA}$ & $\begin{array}{l}\text { Better signal to noise } \\
\text { ratio }\end{array}$ \\
\hline Tube voltage $(\mathrm{kV})$ & 1 with increased $\mathrm{kV}$ & low contrast resolution \\
\hline Pitch & with higher pitch & $\begin{array}{l}\text { low spatial resolution } \\
\text { low signal to noise ratio }\end{array}$ \\
\hline Slice thickness & $\checkmark$ with thicker slice & $\begin{array}{l}\text { 1ess noise } \\
\text { partial volume artefacts } \\
\text { \& low spatial resolution }\end{array}$ \\
\hline Scan length & 1 with scan length & $\checkmark$ risk for motion artefacts \\
\hline Beam filtration & blocks soft x-rays & $\begin{array}{l}1 \text { less scatter } \\
\text { lower contrast }\end{array}$ \\
\hline
\end{tabular}

\section{Image reconstruction}

Iterative reconstruction (IR) was available initially in the infancy of CT back in the 1970's and was denoted the name Algebraic reconstruction technique (ART) [22, 38, 39]. Due to an increase in the amount of image data produced by $\mathrm{CT}$ and the longer reconstruction times, its use in clinical practice was limited. ART was soon replaced with a faster real-time analytical reconstruction method using Filtered back-projection (FBP) technique [22]. FBP has been the standard reconstruction method during the past 40 years but the expanding clinical use of CT and concerns about the increase in associated absorbed dose and artefacts has highlighted limitations of its continued use in clinical practice [22, 38]. Increases in computing power has made the re-emergence of the IR algorithms possible thus improving the performance of image reconstruction. The basic principles of the reconstruction methods used in CT are described in the following sections.

\section{Filtered back projection (FBP)}

In the Filtered back-projection, the measured projection data (raw data) is convolved with a reconstruction kernel (filtration) and back-projected to obtain the reconstructed image (Figure 2). The type of reconstruction kernel influences the image quality characteristics with trade-offs in spatial resolution and image noise. For an increase in spatial resolution, a high pass filter (reconstruction kernel) is used and the resulting images are very 
noisy. A smoothing, low-pass filter will decrease noise at the expense of spatial resolution. The standard FBP typically assumes an infinite small size of the focal spot, pencil beam x-ray geometry, intensity measurement at central point of each detector element and monoenergetic photons [22, 40]. Deviations from these assumptions lead to inaccuracies in reconstructed images such as the beam hardening, streak artefacts and smoothing.

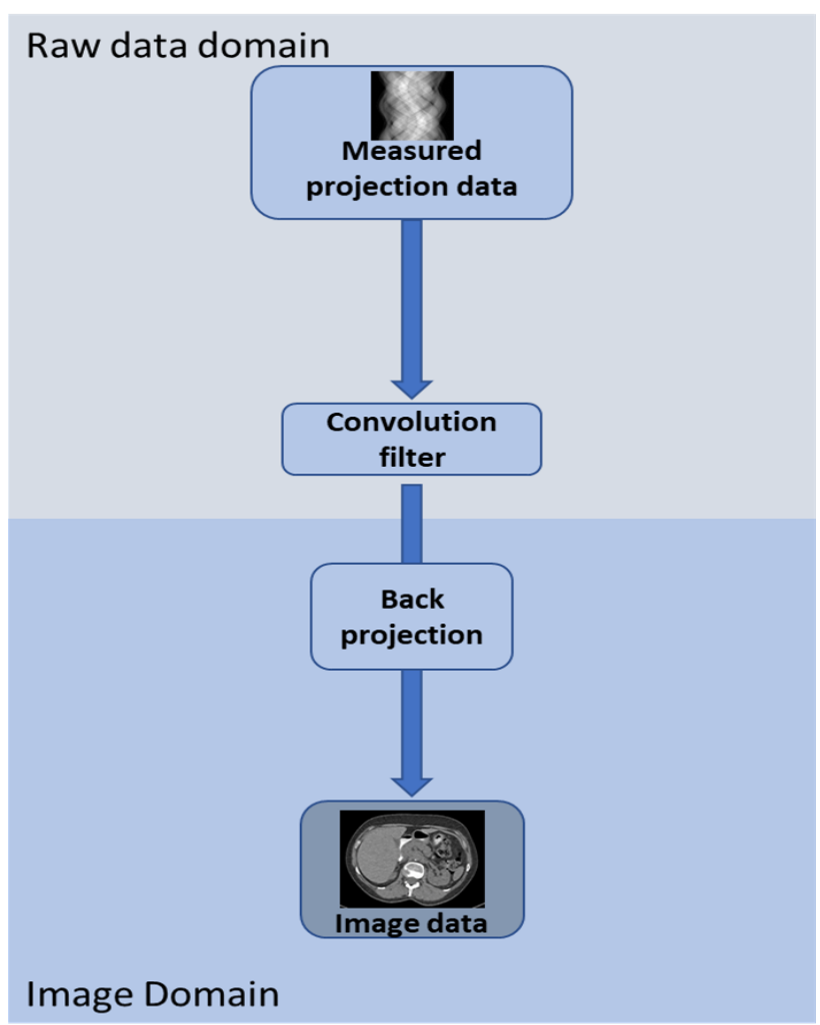

Figure 2. Schematic diagram showing basic principles of conventional filtered back projection (FBP)

A modification of the FBP to a spiral (helical) projection geometry is known as a Weighted filtered back projection (WFBP). Compared to $2 \mathrm{D}$ FBP, the WFBP is an approximate method; it causes artefacts [41].

\section{Iterative reconstruction (IR)}

An IR algorithm consists of three basic steps [42, 43], see Figure 3:

1. A forward projection of the volumetric object estimate creates artificial raw data. 
2. The artificial raw data are compared to the real measured raw data in order to compute an updated image.

3. The updated image is then back projected onto the current volumetric image.

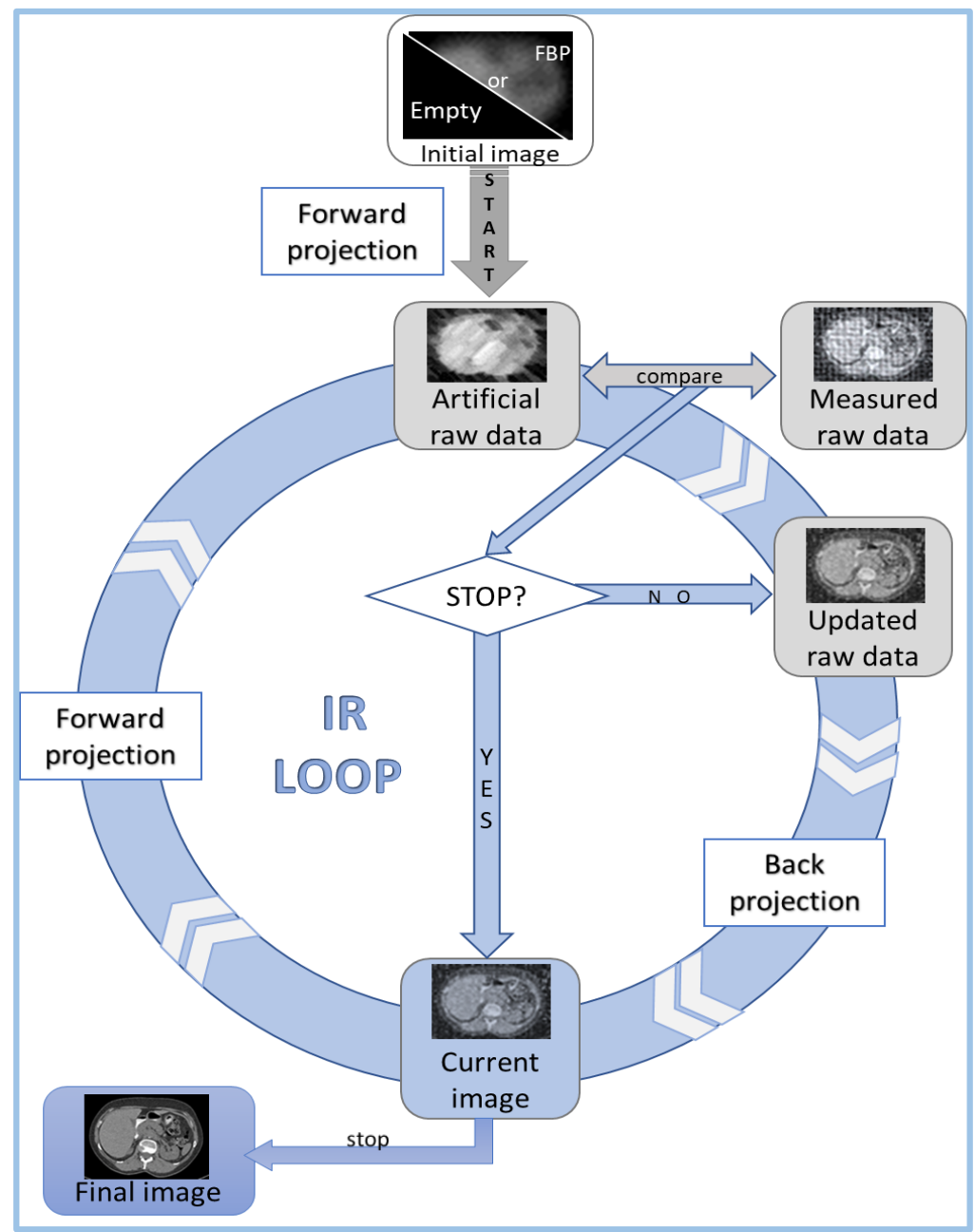

Figure 3. Schematic diagram of the basic principles of iterative reconstruction (IR) algorithm used in Computed Tomography.

Reproduced from Beister et al. Iterative reconstruction methods in X-ray CT. Phys Med. 2012;28(2):94-108 [42] and altered/adapted with permission from Elsevier.

These steps form the iterative loop and are repeated iteratively, until the reconstructed and measured values are within acceptable limits of the predefined image quality setting for the actual protocol on the CT system. The main advantage of IR methods is better reduction of noise and 
artefacts compared to FBP or WFBP. Since reduction in noise allows for lower radiation doses, this outcome is of particular interest in optimisation. There are several commercial IR algorithms available today from all major manufacturers of CT systems. The function and mechanism of these algorithms are vendor specific based on the properties of the imaging system and are classified into two main groups depending on their functional properties; statistical/hybrid and model-based iterative reconstruction algorithms [40, 42, 43, 44].

For model-based IR several geometric, optic and system models are incorporated to correct for artefacts and image degrading effects. In the following sections, statistical (SAFIRE) [45] and model-based (ADMIRE) [46] algorithms available on Siemens systems are described.

\section{Sinogram affirmed iterative reconstruction (SAFIRE)}

SAFIRE is an iterative reconstruction algorithm that incorporates statistical modelling and rough modelling of the projection rays [45]. It is available in 5 strengths, where the level of noise reduction and noise texture depends on the strength used for preferred image quality requirements. Strength 1 images are noisy compared to the smoother appearance of images reconstructed with strength 5 [45]. Denoising is performed in both the image and raw data domains to speed up the reconstruction process providing routinely acceptable reconstruction times that are comparable to that of FBP [40].

In SAFIRE, a CT image is created by applying a weighted FBP (WFBP) to the measured raw data from the CT system. New simulated raw data generated by a forward projection of this CT image, are then compared to the measured data to basically correct and remove artefacts introduced by the imprecise nature of the FBP reconstruction (loop 1) (Figure 4). Loop 1 also compares projection data of adjacent projections to identify noisy or photon-starved projections by means of a dynamic raw-data based noise model [45].

The regularisation loop (loop 2) separates image information from noise, based on an advanced anisotropic spatially variant image noise model which analyses the statistical significance of the sinogram raw data contribution to each image pixel. The detected noise is iteratively removed to improve image quality with reduction of image noise in low contrast areas and enhancing the spatial resolution in objects with high contrast [45, 47]. This process is repeated a number of times depending on the exam type. Loop 2 takes less time to execute than the loop 1 (Figure 4). 


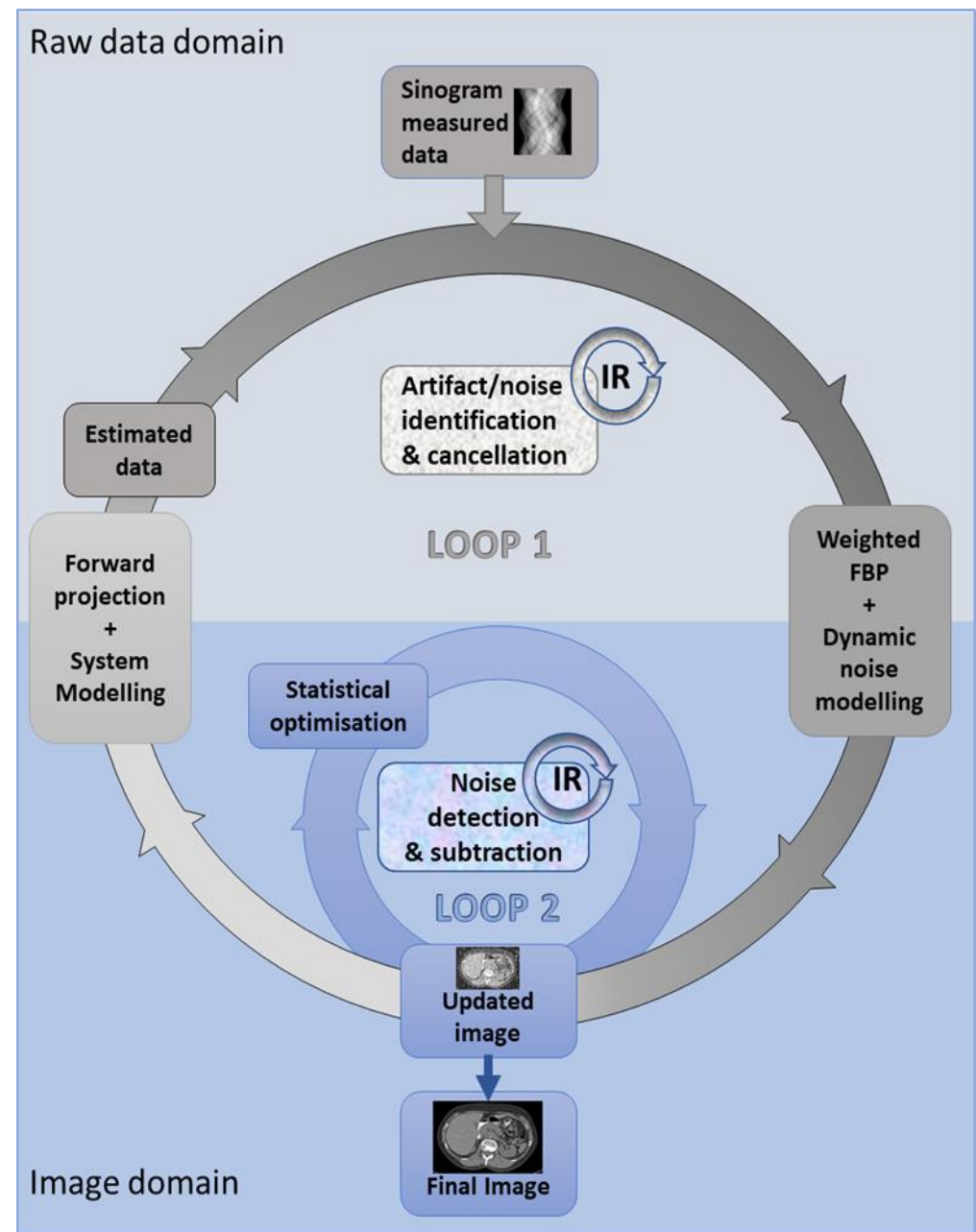

Figure 4. Basic principles of Sinogram affirmed iterative reconstruction (SAFIRE). Loop 1 is the correction loop and loop 2 the regularisation loop. IR represents iterative reconstruction.

Reproduced from White Paper SAFIRE Sinogram affirmed iterative reconstruction [45] and altered/adapted with permission from Siemens Healthineers.

\section{Advanced modeled iterative reconstruction (ADMIRE)}

ADMIRE is a model-based iterative reconstruction algorithm (MBIR). It can be described as a successor to SAFIRE. The technological advances and improvements specifically related to ADMIRE are:

i. Advanced statistical weighting of all projections in the raw data domain.

ii. Advanced regularisation which intelligently separates the noise from actual anatomical structures in the image. 
iii. More complete modelling of the CT geometry and scanner components and characteristics such as detector type, size and flying focal spot to improve spatial resolution and reduce spiral artefacts [46].

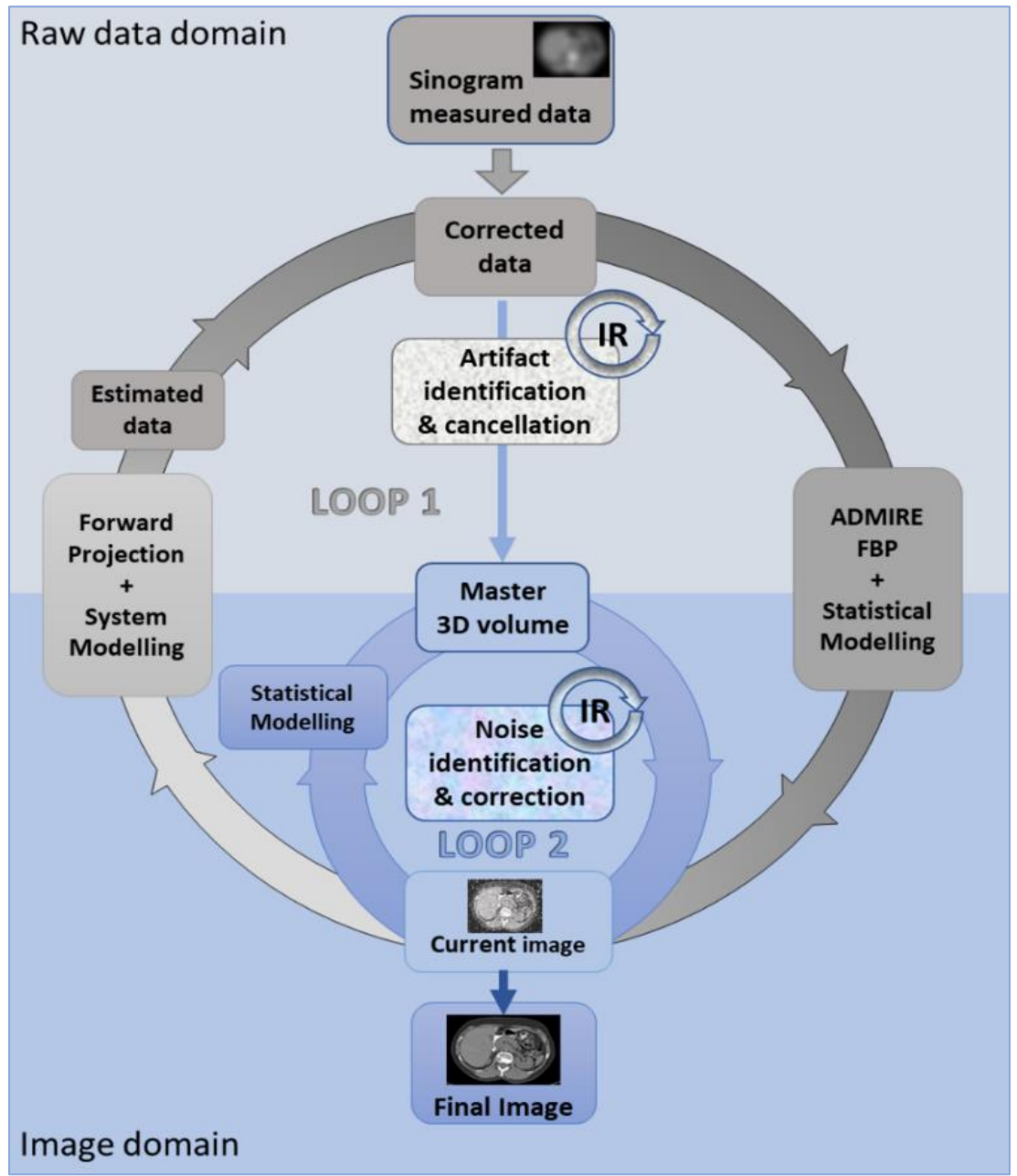

Figure 5. Basic principles of Advanced modeled iterative reconstruction (ADMIRE). Loop 1 is the correction loop for artefact and some noise reduction and loop 2 the statistical optimisation loop primarily for noise reduction. Reproduced from White Paper ADMIRE Advanced modeled iterative reconstruction [46] and altered/adapted with permission from Siemens Healthineers.

Similar to the SAFIRE iterative reconstruction method, it is available in 5 strengths and implements two iterative loops in the reconstruction process. ADMIRE reconstruction starts with a limited number of iterations 
in loop 1 to reduce cone-beam artefacts using a preconditioning filter, and to a lesser extent noise, by means of the statistical weighting in the rawdata domain (Figure 5). Only a small number of iterations are required in the first loop since preconditioning accelerates the system geometrical modelling process of artefact removal. Iterations in the image domain (loop 2) are performed to finalise the statistical optimisation (noise reduction) $[46,48]$. To speed up the iterative process, statistical modelling from the raw data domain is translated to the image domain hence rendering the heavy repeated forward and back projections unnecessary. Consecutive iterations are transformed into a comparison of the "current" data with master $3 \mathrm{D}$ volume instead of the virtual raw data and measured data sets, thus preserving the natural anatomical impression in the images [48].

\section{Image reformatting methods}

In CT, images are obtained primarily in the transverse plane and are available in digital form. This facilitates direct post-processing of the scanned volume by computer algorithms. MDCT provides near isotropic large volume, high resolution images that can be reconstructed into 2- and 3-D displays such as multi-planar reformation (MPR), maximum intensity projection (MIP) and $3 \mathrm{D}$ volume rendering technique (VRT) with a spatial resolution similar to that of the transverse images [22]. The reconstructions are of superior quality enabling a significant improvement in the diagnostic approach when interpreting the large datasets and presenting images to the clinicians in a form they are more familiar with. MPR techniques can further be used to generate thick slices (slabs) from thin slices thus reducing the noise level and possibly also improving the visualisation of anatomical structures present in several slices.

\section{Image quality evaluation}

To determine acceptable diagnostic quality in CT, reliable methods are necessary to evaluate image quality in the resulting images [49]. There are two different approaches to evaluate image quality; the first is physical measurements of objective image quality in phantoms often used for quality assurance and to optimise scanner settings to achieve lowest possible radiation exposure while maintaining the visibility of details in the image. The second approach is visual image quality evaluation by radiologists, performed on clinical patient images rating the quality of the images on a simple scoring scale. The former is relatively easy to perform but since the clinical aspect of the radiologist's true environment is absent, the value of this evaluation limits the implementation of the results directly into clinical practice. Visual assessment, on the other hand, is advantageous as it mirrors the working environment of the radiologists. However, depending on 
the approach, the visual assessment methods can be more or less complex and time consuming.

\section{Objective evaluation}

Objective evaluation is performed on, either phantoms under known conditions or clinical patient images to characterise the physical properties of the image using quantitative measurements such as standard deviation (SD) of the noise, spatial resolution, contrast-to-noise ratio (CNR) and noise power spectrum (NPS).

\section{Noise (SD)}

In CT imaging, noise originates from imaging system electronics, anatomy of the patient and due to statistical fluctuations of the absorption of $x$-ray photons in the image detector, called quantum noise. An increase in image noise may deteriorate image quality. Noise is characterised as the standard deviation (SD) of HUs within a ROI [50].

\section{Spatial resolution}

The spatial resolution or high contrast resolution of a CT system is its ability to resolve small independent objects in close proximity to one another. It can be measured using phantoms with resolution bar-patterns. A more robust and objective evaluation of spatial resolution can be obtained by the modulation transfer function (MTF) involving complex calculations [51].

\section{Contrast-to-noise ratio (CNR)}

Contrast-to-noise ratio is used to determine the low-contrast resolution. Low-contrast resolution is the ability of a CT system to distinguish between two adjacent structures with similar characteristics as separate entities. The CNR is an objective assessment of the contrast between the two structures expressed as a fraction of noise [51].

\section{Noise power spectrum (NPS)}

Although SD is a useful and quick indicator of noise, it is a simple metric and provides no information about the spatial frequency characteristics of the noise. The image reconstruction method used affects the noise frequency distribution in CT and hence the NPS is a more appropriate method to measure noise when comparing two different reconstruction algorithms such as FBP and IR. The NPS is calculated from ROI in a homogenous phantom and measures the frequency content of the noise variation of an image providing a more complete description of the noise texture and distribution [50]. 


\section{Visual evaluation}

The most important aspect of diagnostic imaging is the clinical image quality. The diagnostic process culminates in human assessment of the anatomical and pathological information produced by the imaging modality and is primarily to confirm disease but also - though with limited accuracy - to rule out injury or disease in the individual patient. In order to optimise and evaluate imaging methods, there are several different approaches to subjectively measure image quality $[49,52]$. These methods simulate the clinical diagnostic process and include visual grading experiments such as receiver operating characteristic (ROC) analysis [53], visual grading analysis (VGA) [49], visual grading characteristic (VGC) [54] analysis and visual grading regression (VGR) [55]. In the following sections a brief description of the first three methods and a more detailed description of VGR, which was the principal method used in this thesis, are presented.

\section{Receiver operating characteristic (ROC)}

Diagnostic accuracy is measured using the ROC analysis method which is considered the golden standard for measuring image quality in visual experiments involving detection of known pathology. ROC analysis is derived from the signal detection theory translated to the clinical counterpart as the detection of pathological cases (signal) against a background of normal cases (noise) [49]. The validity of the test is described by two components; sensitivity and specificity. The sensitivity or true positive fraction (TPF) component describes the probability of a pathological examination being determined by the observer as pathological. The specificity or true negative fraction (TNF) component describes the probability of a normal examination determined as being healthy by the observer. Sensitivity is inversely related to the specificity in that when sensitivity increases, the specificity decreases. The ROC curve is generated by plotting the TPF as a function of the false positive fraction (FPF) which is derived from subtracting the specificity from the integer 1 (1-specificity) [56]. The area under the curve $\left(\mathrm{AUC}_{\mathrm{ROC}}\right.$ ) denotes the validity of the diagnostic test and has a value between $\mathrm{O}$ and 1 . The value of 1.0 corresponds to perfect detection while a value of 0.5 represents a detection that is purely due to chance [57].

There are some limitations concerning experiments using standard ROC methodology;

i. Knowledge of the ground truth is required.

ii. Ground truth is often not readily available.

iii. Multiple tumours/lesion in the same image cannot be handled in lesion detection methodology.

iv. Displayed pathology must be close to the limit of detectability by the reader i.e. not too obvious or too difficult to assess. 
v. It is dependent on the readers' ability to detect and correctly interpret the visible pathology.

For the above reasons a standard ROC study is a time-consuming and costly method that requires a large number of cases to produce statistically significant results and may not be a practical choice for optimisation purposes [58].

\section{Visual grading experiments}

There are several reasons as to why visual grading experiments have become an established method when optimising and evaluating clinical image quality in medical imaging. A high practical validity can be assumed as the assessment accounts for contribution of all technical components of the imaging chain in reproducing image structures including involvement of experienced observers [52].

i. Image quality assessment is based on visualisation of clinically relevant anatomical structures which are selected and defined using established standards such as the European Guidelines of quality criteria [59].

ii. Compared to ROC studies, visual grading studies are easier to perform as almost any image can be used in the evaluation.

iii. Participating observer time workload is moderate, hence multiple observers can participate leading to an increase in statistical power of the result $[58,60]$.

iv. In special cases, visual grading experiments have shown equivalent agreement results in detection studies using human observers and with advanced physical image quality calculations [52].

Since visual grading studies are relatively fast, effective and require less resources than a ROC study, they are economically justifiable [52]. In contrast to ROC methods, visual grading experiments focus on the reproduction accuracy of normal anatomy and assume that if visibility and/or reproduction of a certain anatomical structure is possible, then the same applies to pathology [58]. However, it is generally not known to what extent this assumption is true or not.

Visual grading studies evaluate certain well-defined image criteria rated by observers on an ordinal scale. There are two ways to perform a visual grading study:

- In absolute grading, the observer evaluates and rates certain image criteria without a reference image using an absolute scale typically of 4 to 7 points. 
- Relative grading requires one or several reference images where the observer compares quality of the test image with the corresponding reference image using certain image criteria. The study can be conducted by rating the images one at a time or simultaneous viewing of two images. The latter increases the sensitivity of subtle differences in image quality when comparing two images. A typical ordinal scale of 3,5 , or 7 points is used to categorise the observer rating.

\section{Grading of image quality}

Clinical studies involving patient images focus on the evaluation of anatomical structures, which are easy to describe as they have a uniform appearance compared to pathological structures [60]. The highest diagnostic quality is defined as that which enables the observer to accurately report diagnostically relevant structures and features. In visual grading studies, the assessment of image quality is usually based on established quality standards such as the European Guidelines for quality criteria [59], which are available for a variety of examinations. If identical standards are followed in clinical studies, the results from different studies can to a certain degree be compared [6o]. The European quality criteria for CT were developed by an international group of well-established radiologists and physicists and is considered a valid instrument for assessing image quality . Examples of image criteria used for an abdominal CT examination are presented in Table 2.

Table 2. Examples of image criteria used in image quality evaluation of an abdominal CT examination

\section{Criterion}

Visually sharp reproduction of the liver parenchyma

Visually sharp reproduction of the pancreas contour

Visually sharp reproduction of the contours of the kidneys \& proximal ureters

Reproduction of contours of lymph nodes $<15 \mathrm{~mm}$ in diameter

In visual grading studies, the observer is allowed to state his/her confidence regarding the fulfilment of a given criterion on an ordinal scale.

In absolute studies, the observers' opinion on the visibility of a certain feature is obtained with individual assessment of actual image, one at a time without a reference image. In relative studies, the observers' opinion on the visibility of a certain feature is obtained with either individual assessment of actual image, one at a time compared to a reference image, or simultaneous viewing of two images displayed on side-by-side monitors. In image pair comparison studies the quality of the target structure of the test image is compared to the corresponding structure in the reference image. 
To categorise the observer decision, grading scales of Likert-type with 3- up to 7-points are used. Examples of 5-point grading scales used for the different types of visual grading studies are shown in Table 3.

Table 3. Examples of grading scales used in visual grading studies

\begin{tabular}{|l|}
\hline A. Simple visual grading study \\
\hline 1. Criterion is not fulfilled \\
2. Criterion is probably not fulfilled \\
3. Indecisive \\
4. Criterion is probably fulfilled \\
5. Criterion is fulfilled \\
\hline B. Absolute visual grading study \\
\hline 1. Poor image quality \\
2. Restricted image quality \\
3. Sufficient image quality \\
4. Good image quality \\
5. Excellent image quality \\
\hline C. Relative visual grading study - single image compared to refer- \\
ence image \\
\hline 1. Test image clearly inferior to reference image \\
2. Test image somewhat inferior to reference image \\
3. Test image equal to reference image \\
4. Test image somewhat superior to reference image \\
5. Test image clearly superior to reference image \\
\hline D. Relative visual grading study - simultaneous pairwise compari- \\
son \\
\hline +2 Image on right monitor is better than image on left monitor \\
+1 Image on right monitor is probably better than image on left monitor \\
o Both images are equivalent \\
-1 Image on left monitor is probably better than image on right monitor \\
-2 Image on left monitor is better than image on right monitor \\
\hline
\end{tabular}

\section{Observer bias}

Observer bias is introduced through various sources in visual grading studies due to its dependence on human observers when subjectively evaluating image quality. To minimise or counteract the influence of such bias, knowledge about such sources is essential when planning and executing the study [51]. Adaptation bias and recognition bias are two important classifications of observer bias. The former occurs when differences in image appearance and noise texture influences the observer preference in favour of the more familiar image type and may contribute to rejection of a new method or equipment. The latter is typical phenomenon when the intention to completely blind the observer to a certain evaluation variable fails due to some unusual characteristic.

Birkelo et al [61] in their study on tuberculosis case findings in 1947, were the first to make two striking observations. The first is that 
experienced radiologists are not consistent with one another (inter-observer reliability) and the second that there is variation in perception for the same observer (intra-observer reliability) when assessing the same images at different points in time. This variation in perception of image quality among radiologists could be due to the difference in viewing strategies employed and depending on the approach used, these are classified as drilling or scanning [62]. Other factors such as experience and global/focal search patterns also influence perception [62]. To overcome this diversity in perception, an adequate number of observers are required to produce representative results.

It is also advantageous to ensure the observers receive appropriate training to achieve similar understanding of the task at hand before the subjective evaluation of image quality is performed. Statistical software that include random effects can also be used to account for this variation [55].

\section{Data analysis of visual grading data}

For analysing data from visual grading experiments, at least three different approaches have been proposed.

\section{Visual grading analysis (VGA)}

The results of a VGA study are summarised in the VGA score (VGAS):

$$
\mathrm{VGAS}=\frac{\sum_{o, I} S_{c}}{N_{i} N_{o}}
$$

where $S_{c}=$ individual scores for observer $(O)$ and image $(I), N_{i}=$ total number of images, and $N_{o}=$ total number of observers.

Analysis of variance (ANOVA) is used to calculate the significance of differences. The mathematical and statistical validity of the VGA score is questionable since it assumes normal distribution of the data and characterises the scale steps as an interval scale measure. However, since data are defined on an ordinal scale, which cannot be transformed into well-defined numerical values, the ANOVA is considered to be an inappropriate statistical method [49, 6o]. VGA will not be further considered here.

Two analysis methods avoiding these statistical problems will be presented in the following sections.

\section{Visual grading characteristic (VGC) analysis}

Image criteria scoring (ICS) is a simple visual grading method in which the observer rating is a dichotomous decision as to whether the investigated 
criteria are fulfilled or not. Image criteria scores (ICS) are evaluated using a parametric statistical method to calculate the proportion of known fulfilled criteria [54]. The fraction of fulfilled criteria is defined as [63]

$$
\mathrm{ICS}=\frac{\sum_{i=1}^{I} \sum_{c=1}^{C} \sum_{o=1}^{O} F_{I, C, O}}{I \times C \times O}
$$

where $F_{I, C, O}=$ fulfilment of criterion $C$, for image $I$, and observer $O, I=$ number of images, $C=$ number of criteria, $O=$ number of observers.

The basic visual grading characteristic (VGC) study is an expanded image criteria (IC) fulfilment study or a relative or absolute VGA study. VGC is an analysis method developed by Båth and Månsson [54] to address the statistical problems of VGA. VGC analysis fulfils the requirement of a nonparametric, rank-invariant statistical method required to analyse the observer visual grading scores using multiple ordinal scale steps. VGC also deals with the visual grading data in a similar fashion to that of the ROC data. The VGC analysis is performed in a stepwise fashion:

- Step 1 - the number of observer rating scores for each grading stage for the compared modalities are used to create a frequency table $(2 \mathrm{x}$ $n$ frequency table where $n=$ number of rating categories)

- Step 2 - data points representing the VGC curve are calculated from the table in the first step with each point defined by the cumulative frequencies of the categories divided by the total number of decision scores.

- Step 3 - the origin of the VGC-curve is defined as "o" and each point obtained by plotting the cumulative frequencies of the corresponding categories for the two compared systems against each other. The last point is defined as " 1 " as it includes all decisions.

- Step 4-Software intended for use with ROC analysis can be used for plotting the VGC-curve which is a measure of the ICS for the evaluated system as a function of the ICS of the other system. The calculation of the area under the curve ( $\mathrm{AUC}_{\mathrm{VGC}}$ ) is then used to measure the difference in image quality between the two systems [6o].

Somewhat analogue to the ROC curve, an $\mathrm{AUC}_{\mathrm{VGC}}$ of 0.5 indicates that the two systems are equal in image quality, an $\mathrm{AUC}_{\mathrm{VGC}}<0.5$ indicates a higher image quality for the reference system and an $\mathrm{AUC}_{\mathrm{VGC}}>0.5$ indicates the image quality in the evaluated system is higher [52].

An expanded version of the VGC includes the multiple-reader multiplecase (MRMC) option where potential bias resulting from individual differences between study patients or observers (random effects) can be accounted for [64]. A limitation of VGC is that it cannot manage analysis involving several independent variables [55], which has led to the 
development of another analysis method (VGR) based on principles of ordinal logistic regression $[55,65]$.

\section{Visual grading regression (VGR)}

Visual grading regression was developed by Smedby and Fredriksson [55] building on ordinal logistic regression in instances when a researcher is interested in simultaneous assessment of the effect and interaction of several factors that potentially influence the grading of the images. Examples of such factors are variables such as choice of equipment, post-processing methods and acquisition settings.

In VGR, the ordinal visual grading scores are analysed by applying ordinal logistic regression to observer ratings. In the ordinal logistic regression, the observer and patient identities, which are not of primary interest for the researcher, should be treated as random effects to control for the variations between individuals from these groups [65]. There are some differences in the organisation and analysis of data between single-image and image-pair experiments. Examples of hypothetical data organisation for each type of experiment are presented in Tables 4A and 4B [55].

Table 4A. Organisation of data from single-image rating experiment with category label coding.

\begin{tabular}{|c|c|c|c|c|c|}
\hline Observation no & Im & PP & $\mathbf{P}$ & $\mathbf{O}$ & Score \\
\hline 1 & $\operatorname{Im} 1$ & PP1 & $\mathrm{P}_{1}$ & O1 & 1 \\
\hline 2 & $\operatorname{Im} 1$ & $\mathrm{PP}_{2}$ & $\mathrm{P} 1$ & O1 & 2 \\
\hline 3 & $\operatorname{Im} 1$ & $\mathrm{PP}_{3}$ & $\mathrm{P} 1$ & $\mathrm{O} 1$ & 1 \\
\hline 4 & $\operatorname{Im} 2$ & PP1 & $\mathrm{P} 1$ & $\mathrm{O} 1$ & o \\
\hline 5 & Im2 & $\mathrm{PP}_{2}$ & $\mathrm{P}_{1}$ & $\mathrm{O} 1$ & 3 \\
\hline 6 & $\operatorname{Im} 2$ & $\mathrm{PP}_{3}$ & P1 & $\mathrm{O} 1$ & 2 \\
\hline 7 & $\operatorname{Im} 1$ & $\mathrm{PP} 1$ & $\mathrm{P} 1$ & $\mathrm{O} 2$ & 1 \\
\hline 8 & $\operatorname{Im} 1$ & $\mathrm{PP} 2$ & $\mathrm{P} 1$ & $\mathrm{O}_{2}$ & 3 \\
\hline$\ldots \ldots$ & $\ldots \ldots$ & $\ldots \ldots$ & ...... & ...... & $\ldots \ldots$ \\
\hline 144 & $\operatorname{Im} 2$ & $\mathrm{PP}_{3}$ & P6 & $\mathrm{O}_{4}$ & 3 \\
\hline \multicolumn{6}{|c|}{$\begin{array}{l}\text { Comparison of } 2 \text { types of imaging equipment (Im), } 3 \text { post-processing methods (PP), } 6 \\
\text { patients (P) and } 4 \text { observers (O) yielding } 2 \times 3 \times 6 \times 4=144 \text { observations. } \\
\text { Reprinted with permission from Smedby et al. Visual grading regression: analysing data } \\
\text { from visual grading experiments with regression models, BJR 2010:1-9 doi: } \\
10.1259 / \mathrm{bjr} / 35254923\end{array}$} \\
\hline
\end{tabular}

The basic VGR framework presented in this thesis is as described in Smedby et al (2010) [55]. It is based on the ratio of the probability $(p)$ of an event occurring in relation to the probability of an event not occurring, called the odds of the event denoted by the equation: 


$$
\text { odds }=\frac{p}{1-p}
$$

A logistic function (logarithm of the odds) is used to transform probability:

$$
\operatorname{logit}(p)=\log (\text { odds })=\log \left(\frac{p}{1-p}\right)
$$

resulting in a linear equation, which and in the simplest case, with one independent continuous variable, takes the form:

$$
\operatorname{logit}(p)=a x+b=z
$$

In the more general case, $z$ is the weighted sum of all the independent variables used in the model which are either continuous or dummy variables [55].

The explicit dependence of the probability $p$ on the independent variable $z$ is given by:

$$
p=\frac{1}{1+e^{-z}}
$$

When applying VGR to the data from a visual grading study, the dependent variable $y$ is defined on an ordinal scale representing the score predicted by the model. The ordinal rating scale value $n$ takes on the scale values used, for example, $n$ is 1, 2, 3 or 4 when a 4-point scale is used. For a combination of independent values, the cumulative probability $(P)$ represents the probability of obtaining a visual grading score not greater than $n$ in the equation:

$$
\operatorname{logit}[P(y \leq n)]=z
$$

where $P(A)$ is the probability of the event $\mathrm{A}, y$ is the score assigned by the observers, and $z$ the linear combination of factors assumed to affect perceived image quality [55].

Each of the independent categorical variables are represented by a term with a separate value for each category. For example, if a model includes two variables PP (post processing) and Im (imaging systems), the corresponding terms will be $A_{I m}$ and $B_{P P}$ with $A_{1}$ characterising the $\operatorname{Im} 1$ and $A_{2}$ the Im2. The variables $\mathrm{P}$ (patient) and $\mathrm{O}$ (observer) should be introduced into the model to manage the variation that arises due to differences between the individuals in these groups. The probability of obtaining a value not greater than $n$ is given by: 


$$
\operatorname{logit}[P(y \leq n)]=z=A_{I m}+B_{P P}+D_{p}+E_{O}-C_{n}
$$

For image-pair assessments the corresponding equation is:

$$
\operatorname{logit}[P(y \leq n)]=A_{I m-L}-A_{I m-R}+B_{p p-L}-B_{p p-R}+D_{P}+E_{O}-C_{n}
$$

with labels $-L$ and $-R$ corresponding to left and right image in each pair respectively [55].

Table 4B. Organisation of data from image-pair rating experiment with category

\begin{tabular}{|c|c|c|c|c|c|c|c|}
\hline Observation no & Im-L & Im-R & PP-L & PP-R & $\mathbf{P}$ & $\mathbf{O}$ & Score \\
\hline 1 & $\operatorname{Im} 1$ & $\operatorname{Im} 2$ & $\mathrm{PP} 1$ & PP1 & P1 & $\mathrm{O} 1$ & $\mathrm{O}$ \\
\hline 2 & $\operatorname{Im} 1$ & $\operatorname{Im} 2$ & $\mathrm{PP} 2$ & $\mathrm{PP}_{2}$ & $\mathrm{P}_{1}$ & $\mathrm{O} 1$ & 1 \\
\hline 3 & $\operatorname{Im} 1$ & $\operatorname{Im} 2$ & $\mathrm{PP}_{3}$ & $\mathrm{PP}_{3}$ & P1 & $\mathrm{O} 1$ & 1 \\
\hline 4 & $\operatorname{Im} 1$ & $\operatorname{Im} 1$ & $\mathrm{PP} 1$ & $\mathrm{PP}_{2}$ & $\mathrm{P} 1$ & $\mathrm{O} 1$ & -2 \\
\hline 5 & $\operatorname{Im} 1$ & $\operatorname{Im} 1$ & $\mathrm{PP} 2$ & $\mathrm{PP}_{3}$ & $\mathrm{P} 1$ & $\mathrm{O} 1$ & o \\
\hline 6 & $\operatorname{Im} 1$ & $\operatorname{Im} 1$ & $\mathrm{PP}_{3}$ & $\mathrm{PP} 1$ & $\mathrm{P}_{1}$ & O1 & 1 \\
\hline 7 & $\operatorname{Im} 2$ & $\operatorname{Im} 2$ & PP1 & $\mathrm{PP} 2$ & $\mathrm{P} 1$ & $\mathrm{O} 1$ & -2 \\
\hline 8 & $\operatorname{Im} 2$ & $\operatorname{Im} 2$ & $\mathrm{PP} 2$ & $\mathrm{PP}_{3}$ & $\mathrm{P}_{1}$ & O1 & 1 \\
\hline 9 & $\operatorname{Im} 2$ & $\operatorname{Im} 2$ & $\mathrm{PP}_{3}$ & $\mathrm{PP} 1$ & $\mathrm{P} 1$ & $\mathrm{O} 1$ & 1 \\
\hline 10 & Im2 & $\operatorname{Im} 1$ & $\mathrm{PP} 1$ & $\mathrm{PP} 1$ & $\mathrm{P} 1$ & $\mathrm{O} 2$ & 1 \\
\hline 11 & $\operatorname{Im} 2$ & $\operatorname{Im} 1$ & $\mathrm{PP} 2$ & $\mathrm{PP} 2$ & P1 & $\mathrm{O} 2$ & o \\
\hline 12 & $\operatorname{Im} 2$ & $\operatorname{Im} 1$ & $\mathrm{PP}_{3}$ & $\mathrm{PP}_{3}$ & $\mathrm{P} 1$ & $\mathrm{O} 2$ & 1 \\
\hline 13 & $\operatorname{Im} 1$ & $\operatorname{Im} 1$ & PP1 & PP1 & $\mathrm{P}_{1}$ & $\mathrm{O} 2$ & 1 \\
\hline$\ldots \ldots$. & $\ldots \ldots$. & & $\ldots \ldots$ & & ....... & ....... & $\ldots \ldots$ \\
\hline 216 & $\operatorname{Im} 2$ & $\mathrm{Im} 2$ & PP1 & $\mathrm{PP}_{3}$ & P6 & $\mathrm{O}_{4}$ & -1 \\
\hline \multicolumn{8}{|c|}{$\begin{array}{l}\text { Variable names ending in }-\mathrm{L} \text { refer to left image in each image pair, }-\mathrm{R} \text { to the right } \\
\text { one. Comparison between imaging methods are represented by observations } 1-3 \text { and } \\
\text { between post-processing methods by observations } 4-9 \text {. } \\
\mathrm{Im}=\mathrm{imaging} \text { equipment, } \mathrm{P}=\mathrm{p} \text { atient, } \mathrm{O}=\mathrm{observer} \text {. } \\
\text { Reprinted with permission from Smedby et al. Visual grading regression: analysing data } \\
\text { from visual grading experiments with regression models, BJR 2010:1-9 doi: } \\
10.1259 / \mathrm{bjr} / 35254923\end{array}$} \\
\hline
\end{tabular}
label coding.

An alternative method of coding is assigning dummy values to each variable category which can only have the values of 0 or 1 for single-image studies. Each possible category is then allocated a column in the table with the value 1 in the column for actual category and o in the other columns. In pairwise comparison, values are no longer restricted to 0 and 1 , as a value of 1 is given when the feature is present only in the left image and -1 when 
the feature is present only in the right image and o when there is no difference between the two images with respect to the independent variable assessed. The conversion to dummy variables is automatically available with most modern software packages [55].

\section{Statistical analysis (VGR)}

Numerical results of the ordinal logistic regression are provided by the statistical software used in the data evaluation process. The logistic function of the cumulative probability is the weighted sum ( $z$ ) given by the following equation:

$$
\operatorname{logit}[P(y \leq n)]=\log \left(\frac{P(y \leq n)}{1-P(y \leq n)}\right)=z=\beta_{0}+\beta_{1} x_{1}+\beta_{2} x_{2}+\beta_{3} x_{3}+\cdots \beta_{i} x_{i}+D_{p}+E_{O}-C_{n}
$$

where $\beta_{o}$ is the intercept (the value of $z$ when all independent variables are zero), and $\beta_{1}, \beta_{2} \ldots . . \beta_{i}$ represent the coefficients to be estimated. The independent variables are denoted as $x_{1}, x_{2} \ldots \ldots x_{3}$ and the parameters $D_{p}, E_{O}$ and $C_{n}$ are the specific values for patient, observer and quality level respectively [66].

The effects of each independent variable on the dependent variable are described by the regression coefficients. A positive regression coefficient indicates that the explanatory variable increases the probability of the specific outcomes while the opposite is true for a negative regression coefficient value. The influence of variables on the specific probability outcome is indicated to be substantial by a large regression coefficient value. On the other hand, a coefficient value closer to zero indicates a smaller impact on the probability outcome [55].

The visual grading statistical model may include both fixed and random effects. Examples of fixed effects are factors such as tube load, postprocessing parameters, slice thickness etc. Patients and observers, however, are samples from two underlying populations and are per se not interesting as individuals and are therefore treated as random effects [67]. The mixed effects ordinal logistic regression is the method of choice in the statistical analysis software (e.g. the meologit function Stata) [67].

\section{Estimating dose reductions}

VGR, as opposed to ROC methods, can fulfil the purpose of determining potential dose reduction in the optimisation process. The VGR framework estimates potential dose reductions based on experiments involving variation in imaging technique and tube load (mAs) settings, which is considered proportional to the effective dose. The influence of the mAs setting is included in the model and it is assumed that the mAs setting should be 
multiplied by a certain factor in order to achieve a given effect on the perceived image quality. Thus for a given interval, the effect of doubling the mAs setting will always be the same, which is accomplished by introducing a $\log (\mathrm{mAs})$ term in the regression equation [68]. In an example of a singleimage experiment with post-processing applied to some of the images, the regression equation will then read:

$$
\operatorname{logit}[P(y \leq n)]=a \log (\mathrm{mAs})+b \mathrm{PP}+D_{p}+E_{O}-C_{n}
$$

where $a$ and $b$ are coefficients that describe the $\log (\mathrm{mAs})$ and post-processing dependency, respectively. PP represents post-processing as a binary variable ( $1=$ post-processing applied, $0=$ post-processing not applied), $D_{p}$ and $E_{O}$ are terms for individual patients and observers and $C_{n}$ a specific constant for each scoring level $n$.

It is thus possible to estimate the change in $\log (\mathrm{mAs})$ that would give the same change in probability score when post-processing is applied using the equation:

$$
\Delta \log (\mathrm{mAs})=b / a
$$

and hence estimate the value of the relative reduction (RR) in mAs by calculating from the ratio between two regression coefficients [68]:

$$
\mathrm{RR}_{\mathrm{mAs}}=1-e^{(-b / a)}
$$

\section{Radiographers' role in radiation protection}

The role of a radiographer is multifaceted. The main expertise and responsibilities of a radiographer are to perform the whole range of diagnostic imaging techniques and to consequently assess the image quality made available for diagnostic interpretation. The radiographer holds a key position in radiation protection of the patient, public and other staff and has the responsibility of ensuring the dose delivered when acquiring high-quality diagnostic images is kept as low as reasonably achievable [69]. It is important that the radiographer has knowledge to evaluate the indication and justification of the impending examination and can communicate radiation risks to the patients, public and other health workers. A radiographer should have sound knowledge to hold discussions in the patient's best interest and argument for the patient's safety.

The rapid development of diagnostic modalities and continuing expansion of new imaging techniques have led to an increase in volume and complexity of services provided by radiology departments [70]. With this 
development, the character of the radiographers' professional activity has evolved into an independent role demanding increasing expertise not only regarding patient care, technical, radiographic and patient safety related functions but also in pathophysiological aspects of the human body.

Radiology departments spend considerable financial resources in acquiring modern, dose-efficient CT equipment, evaluating it for the purpose of dose management and training radiology staff to improve patient safety [71]. To ensure a favourable benefit-to-risk ratio and best practice in CT, the role of radiation protection requires a multi-professional approach. As part of a multi-disciplinary team, the responsibility of the radiographer in radiation protection when performing CT examinations is outlined in Table 5 .

Table 5. Radiation protection responsibilities of the radiographer in Computed Tomography procedures.

\begin{tabular}{|c|c|c|}
\hline When? & Action & Involvement \\
\hline Before CT & Justification & $\begin{array}{l}\text { Read clinical information } \\
\text { Check for duplicate examinations } \\
\text { Consider risk vs. benefit } \\
\text { Is the exam appropriate? } \\
\text { ALARA? }\end{array}$ \\
\hline During CT & Optimisation & $\begin{array}{l}\text { Read clinical information } \\
\text { Check patient identity } \\
\text { Proper patient positioning } \\
\text { Provide adequate patient information } \\
\text { Patient autonomy withheld } \\
\text { Check scan protocol parameters } \\
\text { Check approx. dose values } \\
\text { Scan field limitation } \\
\text { Correct technical parameters } \\
\text { Modify and adapt to patient needs } \\
\text { Intravenous contrast administration } \\
\text { Produce accurate images } \\
\text { Evaluate image quality }\end{array}$ \\
\hline After CT & Dose management & $\begin{array}{l}\text { Dose registration } \\
\text { Repeat scan? Justification } \\
\text { Reporting dose incidents } \\
\text { Protocol management } \\
\text { Clinical auditing } \\
\text { Contribute towards establishing DRLs } \\
\text { Optimisation }\end{array}$ \\
\hline
\end{tabular}

To maintain patient safety in diagnostic radiology and radiotherapy, the profession should practice radiation protection using the principles of justification, optimisation and ALARA [72]. Justification has previously 
been the responsibility of the referring doctors and radiologists. However, the international society of radiographers and radiology technicians (ISRRT) have issued a statement based on the international basic safety standard (BSS), that radiographers shall also have specified responsibilities as professionals delivering radiation doses. The justification flow chart has been proposed as a decision tool in exercising professional judgement when accepting a referral for exposure [73].

Optimisation applies to individual patients whereby the type of procedure, the dose, other physical imaging parameters, use of contrast media and other drugs must be adapted to the individual specific clinical enquiry. Typical optimisation tools used in CT examinations are appropriate scan length, limiting number of scan series, dose modulation techniques and iterative reconstruction methods to minimise patient dose [72].

Good knowledge and understanding of these parameters facilitate in adapting the procedure to the patient's needs according to the ALARA principle. As a professional, the radiographer is expected to engage in multidisciplinary collaboration to improve and maintain the quality of professional practice and to embrace new technology and contribute to the education and training of students and other staff [74].

Diagnostic radiology is a rapidly changing discipline which brings both excitement and challenge to the radiographer's work. This influences the radiographers' competence, therefore continuous medical education (CME) and personal development (CPD) are necessary to update the professional knowledge and skills in order to serve the patients properly [75]. This is accomplished by adapting present practice to new technologies, developments and circumstances. A culture of continuous learning should be incorporated into practice supported by the employers, professional and national societies. Provision in the form of protected time and resources (by employers) and timely, up-to-date educational opportunities (by professional societies) should be provided for all professionals. Personal learning opportunities such as intra-departmental CPD should be encouraged and ideally be included in work schedules [72]. 


\section{AIMS}

The overall aim of this thesis was to evaluate the dose reduction potential of modern technology features such as iterative reconstruction and postprocessing reformatting methods in order to optimise clinical protocols for abdominal CT and to evaluate the diagnostic value of low-dose abdominal CT.

The specific aim of each paper was as follows:

I. To compare image quality with iterative reconstruction to Filtered back projection in low-dose abdominal CT and to evaluate if further patient dose reduction is possible.

II. To assess visual image quality between Filtered back projection and ADMIRE strengths 3 and 5 (out of 5 ) in abdominal CT, and to estimate the dose-reduction potential of the reconstruction algorithm.

III. To compare CT images from native, nephrographic and excretory phases in CT-urography, using image criteria as well as the detection of positive pathological findings and to explore if reduction of the radiation burden can be achieved in the younger patient groups, patients undergoing repetitive imaging or patients with negative outcomes.

IV. To evaluate the image quality in multiplanar reconstruction (MPR) images with both a subjective and an objective approach in non-enhanced abdominal CT, and to explore the effect of ADMIRE strength and slice thickness on possible dose reduction. 
Visual grading evaluation and optimisation in abdominal Computed Tomography 


\section{METHOD}

This thesis included a total of 140 human subjects (Table 6) in the four image quality evaluation studies, three of which were prospective studies (Papers I, II and IV) and one retrospective study (Paper III). Visual grading experiments to determine the potential dose reductions, were performed with pairwise comparison of image quality in the same patient at different tube loads and reconstructed with different algorithms (Paper I and II) as well as with variations in slice thickness (Paper IV). Paper III, on the other hand, was an absolute assessment of image quality between the three phases of a CT Urography (CTU) protocol to explore the diagnostic value of low-dose abdominal CT.

\section{Research subjects}

The study subjects were recruited among patients referred to the radiology department for clinically indicated low-dose abdominal CT (Paper I), standard dose abdominal CT (Papers II and IV) and CTU (Paper III) examinations. An overview of demographical data for the study subjects is presented in Table 6.

\section{Ethical aspects}

Research ethics approval was obtained from the regional ethical board and the radiation protection committee for all four study subjects to comply with research ethics regulations, especially since subjects in Paper I were exposed to increased radiation with the additional CT examination performed. Written informed consent was obtained for subjects included in Papers I, II and IV. Due to the retrospective nature of the study in Paper III, informed consent was waived. All images used in the four papers were anonymised with respect to the patient identification data to comply with the ethical standards.

\section{Inclusion and exclusion criteria}

Since an additional exposure was performed on study subjects in Paper I, those included in the study were from the age group $>50$ years. Severely ill patients, non-Swedish speaking patients and patients who had undergone more than four CT scans in the past year were excluded. 
For subjects included in Papers II and IV, this ethical dilemma was resolved by using an experimental setup for the dual-source scanner to allow for simultaneous acquisition of 3 datasets at 3 dose-levels in the same patient. Ethical approval was obtained for data collection of 90 patients as the patient fit within the $35 \mathrm{~cm}$ scan field of view of the second detector, could only be determined after the scan was performed.

Paper III included clinically indicated examinations retrieved from the Picture Archiving and Communications System (PACS). Despite the retrospective nature of the study, ethical approval was obtained for publication purposes.

The demographics of all four studies' subjects are presented in Table 6.

Table 6. Overview of study subjects demographic data.

\begin{tabular}{|c|c|c|c|c|}
\hline (2) & Paper I & Paper II & Paper III & Paper IV \\
\hline Number of cases & 45 & 50 & 40 & 25 \\
\hline $\begin{array}{l}\text { Research ethics } \\
\text { approval }\end{array}$ & Yes & Yes & Yes & Yes \\
\hline $\begin{array}{l}\text { Radiation protection } \\
\text { approval }\end{array}$ & Yes & Yes & Yes & Yes \\
\hline $\begin{array}{l}\text { Subject age (years) } \\
\text { Range } \\
\text { Mean } \pm \text { SD }\end{array}$ & $\begin{array}{l}50-95 \\
65 \pm 9.6\end{array}$ & $\begin{array}{c}22-90 \\
64.7 \pm 13.7\end{array}$ & $\begin{array}{c}27-85 \\
62.4 \pm 14.7\end{array}$ & $\begin{array}{c}53-92 \\
71.6 \pm 10.1\end{array}$ \\
\hline Sex & $\begin{array}{c}24 \text { men } \\
21 \text { women }\end{array}$ & $\begin{array}{c}28 \text { men } \\
22 \text { women }\end{array}$ & $\begin{array}{c}28 \text { men } \\
12 \text { women }\end{array}$ & $\begin{array}{c}12 \text { men } \\
13 \text { women }\end{array}$ \\
\hline $\begin{array}{l}\text { Subject BMI } \\
\left(\mathrm{kg} / \mathrm{m}^{2}\right) \\
\text { Range } \\
\text { Mean } \pm \text { SD }\end{array}$ & $\begin{array}{l}17.1-46.8 \\
28.2 \pm 5.6\end{array}$ & $\begin{array}{c}16.4-27.3 \\
22.6 \pm 2.6\end{array}$ & $\begin{array}{c}19.3-38.9 \\
27.2 \pm 4.1\end{array}$ & $\begin{array}{c}17.3-26.2 \\
22.8 \pm 2.1\end{array}$ \\
\hline $\begin{array}{l}\text { CTDI }_{\text {vol }}(\mathrm{mGy}) \\
\text { Range } \\
\text { Mean } \pm \mathrm{SD}\end{array}$ & $\begin{array}{l}1.8-6.6 \\
3.3 \pm 1.0\end{array}$ & $\begin{array}{l}3.9-9.1 \\
6.2 \pm 1.3\end{array}$ & $\begin{array}{c}1.5-14.6 \\
4.2 \pm 2.3\end{array}$ & $\begin{array}{l}4.4-8.3 \\
6.4 \pm 1.2\end{array}$ \\
\hline $\begin{array}{l}\text { DLP }\left(\mathrm{mGy}^{*} \mathrm{~cm}\right) \\
\text { Range } \\
\text { Mean } \pm \mathrm{SD}\end{array}$ & $\begin{array}{c}167-311 \\
148.7 \pm 52.6\end{array}$ & $\begin{array}{c}161-468 \\
292 \pm 70\end{array}$ & $\begin{array}{r}63.8-696.8 \\
202.5 \pm 115.4 \\
\end{array}$ & $\begin{array}{r}194-385 \\
303 \pm 61.8\end{array}$ \\
\hline $\begin{array}{l}\text { SSDE (mGy) } \\
\text { Range } \\
\text { Mean } \pm \text { SD }\end{array}$ & $140.7+52.0$ & $\begin{array}{c}6.6-12.8 \\
8.6 \pm 1.5\end{array}$ & $\begin{array}{c}2.3-18.8 \\
5.1 \pm 2.8\end{array}$ & $\begin{array}{c}6.2-12.2 \\
8.7 \pm 1.3\end{array}$ \\
\hline
\end{tabular}

$\mathrm{BMI}=$ Body mass index, $\mathrm{CTDI}_{\mathrm{vol}}=$ Volume Computer Tomography Dose Index, DLP=Dose

Length Product, SSDE=Size Specific Dose Estimate

\section{CT acquisitions}

The acquisitions performed were clinical protocols for low-dose abdominal CT, standard dose abdominal CT and CTU. Exposure parameters for all four studies were the same as those used for routine clinical protocols in the department at the time. Detailed CT exposure parameters are presented in Table 7. 
Table 7. A summary of CT Protocol parameters for acquisitions performed in each paper.

\begin{tabular}{|c|c|c|c|c|}
\hline & Paper I & Paper II & Paper III & Paper IV \\
\hline CT system & $\begin{array}{c}\text { Siemens } \\
\text { Somatom } \\
\text { Definition } \\
\text { AS }\end{array}$ & $\begin{array}{c}\text { Siemens } \\
\text { Somatom } \\
\text { Force } \\
\text { Dual Energy }\end{array}$ & $\begin{array}{c}\text { Siemens } \\
\text { Somatom } \\
\text { Force } \\
\text { Dual Energy }\end{array}$ & $\begin{array}{c}\text { Siemens } \\
\text { Somatom } \\
\text { Force } \\
\text { Dual Energy }\end{array}$ \\
\hline Type of examination & $\begin{array}{l}\text { Low } \\
\text { dose }\end{array}$ & $\begin{array}{l}\text { Standard } \\
\text { dose }\end{array}$ & Urography & $\begin{array}{c}\text { Standard } \\
\text { dose }\end{array}$ \\
\hline $\begin{array}{l}\text { Number of } \\
\text { acquisitions }\end{array}$ & 2 & 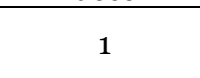 & 3 & 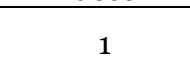 \\
\hline $\begin{array}{l}\text { Tube voltage } \\
(\mathrm{kV})\end{array}$ & 120 & 120 & 120 & 120 \\
\hline \multirow{3}{*}{$\begin{array}{l}\text { Tube current time } \\
\text { product Qref. (mAs) }\end{array}$} & 35 & 42 & 45 & 42 \\
\hline & 50 & 98 & 140 & 98 \\
\hline & - & 140 & 45 & 140 \\
\hline Beam collimation & $64 \times 0.6$ & $96 \times 0.6$ & $96 \times 0.6$ & $96 \times 0.6$ \\
\hline Pitch & 1.2 & 0.6 & 0.6 & 0.6 \\
\hline Rotation time (s) & 0.5 & 0.5 & 0.5 & 0.5 \\
\hline ATCM & Enabled & Enabled & Enabled & Enabled \\
\hline ATVS & Enabled & Disabled & Enabled & Disabled \\
\hline $\begin{array}{l}\text { Contrast } \\
\text { enhancement }\end{array}$ & No & Yes & Yes & No \\
\hline Reconstruction kernel & B26f & Br36 & Br36 & Br36 \\
\hline $\begin{array}{l}\text { Reconstruction } \\
\text { algorithms }\end{array}$ & SAFIRE & ADMIRE & ADMIRE & ADMIRE \\
\hline Phantom acquisition & - & Yes & - & Yes \\
\hline
\end{tabular}

ATCM=Automatic tube current modulation, ATVS=Automatic tube voltage selection, SAFIRE=Sinogram affirmed iterative reconstruction, ADMIRE=Advanced modeled iterative reconstruction.

\section{Study design}

A variety of tube loads and image reconstruction algorithms and methods were used to evaluate the image quality and estimate potential dose reductions in Papers I, II and IV. In paper III, absolute, individual assessment of 3-plane MPR reconstructions for each phase of the CTU protocol were assessed. The comparisons performed are shown in Figures 6 to 9 .

Images were displayed on clinically standardised calibrated DICOM workstations with simultaneous pairwise comparison of axial images (Paper I and II) (Figure 6 and Figure 7) and 3-plane MPR images (Paper IV) (Figure 9) in the same patient. The images were randomly assigned to the right and left monitor. In Paper III the MPR images of each phase of the CTU were displayed and assessed individually. 


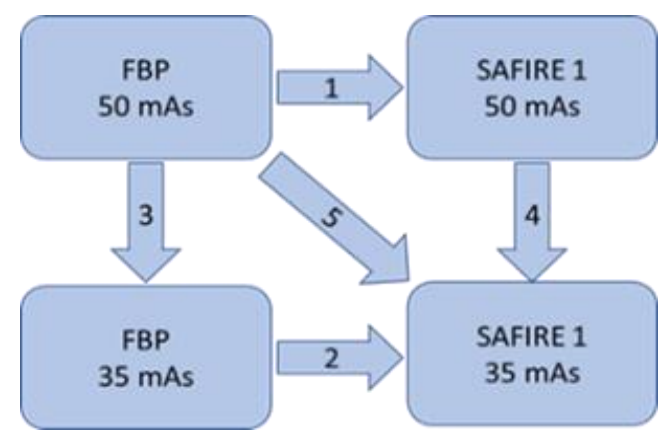

Figure 6. Schematic diagram of pairwise comparisons performed in a lowdose abdominal CT (Paper I). Images were obtained at two tube loads and reconstructed with Filtered back projection (FBP) and Sinogram affirmed iterative reconstruction (SAFIRE) strength 1.

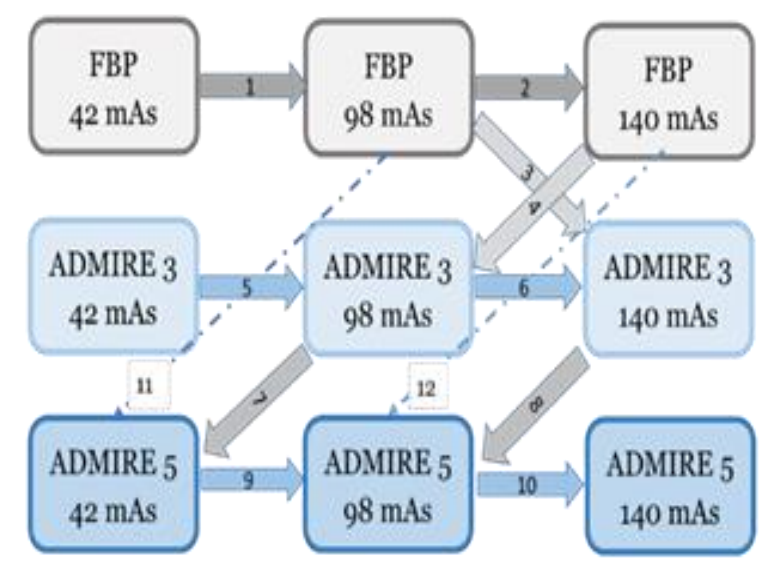

Figure 7. Schematic diagram of pairwise comparisons performed in a standard dose abdominal CT (Paper II). Images were obtained at three tube loads and reconstructed with Filtered back projection (FBP) and Advanced modeled iterative reconstruction (ADMIRE) strengths 3 and 5 .

Image quality assessment was performed independently by a number of observers with varying experience using a number of anatomical criteria from the European guidelines for quality criteria in CT to suit the purpose of each study [59]. Table 8 outlines the study design characteristics, image reconstructions and type of comparisons performed for the individual papers included in this thesis. 
Table 8. Study design characteristics and image reconstruction comparisons for the individual papers

\begin{tabular}{|c|c|c|c|c|}
\hline 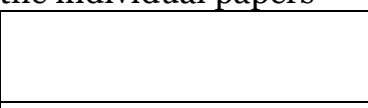 & $\begin{array}{l}\text { Paper } \\
\text { I }\end{array}$ & $\begin{array}{l}\text { Paper } \\
\text { II }\end{array}$ & $\begin{array}{l}\text { Paper } \\
\text { III }\end{array}$ & $\begin{array}{c}\text { Paper } \\
\text { IV }\end{array}$ \\
\hline Study design & Prospective & Prospective & Retrospective & Prospective \\
\hline Type of examination & Low dose & $\begin{array}{c}\text { Standard } \\
\text { dose }\end{array}$ & Urography & $\begin{array}{c}\text { Standard } \\
\text { dose }\end{array}$ \\
\hline Number of criteria & 6 & 6 & 7 & 5 \\
\hline Pathology assessment & Yes & No & Yes & No \\
\hline $\begin{array}{l}\text { Number of comparisons } \\
\text { per patient }\end{array}$ & 5 & 12 & 3 & 20 \\
\hline Number of assessments & 900 & 3000 & 360 & 2000 \\
\hline Type of assessment & Relative & Relative & Absolute & Relative \\
\hline \multirow{2}{*}{ Assessment method } & \multirow{2}{*}{ Visual } & \multirow{2}{*}{ Visual } & \multirow{2}{*}{ Visual } & Visual \\
\hline & & & & Objective \\
\hline Number of observers & 4 & 5 & 3 & 4 \\
\hline $\begin{array}{l}\begin{array}{l}\text { Observer experience } \\
\text { (years) }\end{array} \\
\end{array}$ & $1-22$ & $5-20$ & $8-24$ & $9-25$ \\
\hline Image plane or planes & Axial & Axial & 3 plane MPR & $\begin{array}{l}3 \text { plane } \\
\text { MPR }\end{array}$ \\
\hline Ordinal grading scale & -2 to +2 & -2 to +2 & 1 to 5 & -2 to +2 \\
\hline \multirow{3}{*}{$\begin{array}{l}\text { MPR reconstruction slice } \\
\text { thickness: increment } \\
(\mathrm{mm})\end{array}$} & $5: 2.5$ & $3: 2$ & $3: 2$ & 1:0.5 \\
\hline & & & & $2: 1$ \\
\hline & & & & $3: 2$ \\
\hline \multirow{3}{*}{$\begin{array}{l}\text { Reconstruction algorithm } \\
\text { and strength (1-5) }\end{array}$} & FBP & FBP & \multirow{3}{*}{ ADMIRE 3} & ADMIRE 3 \\
\hline & \multirow{2}{*}{ SAFIRE 1} & ADMIRE 3 & & ADMIRE 5 \\
\hline & & ADMIRE 5 & & \\
\hline \multirow{3}{*}{$\begin{array}{l}\text { Tube load } \\
\text { Q ref mAs }\end{array}$} & 35 & 42 & 45 & 42 \\
\hline & 50 & 98 & 140 & 98 \\
\hline & & 140 & 45 & \\
\hline Coaching session & No & Yes & Yes & Yes \\
\hline
\end{tabular}

Relative assessment=Pairwise comparison, MPR=Multi-planar reconstruction FBP=Filtered Back Projection, SAFIRE=Sinogram affirmed iterative reconstruction, ADMIRE $=$ Advanced modeled iterative reconstruction 


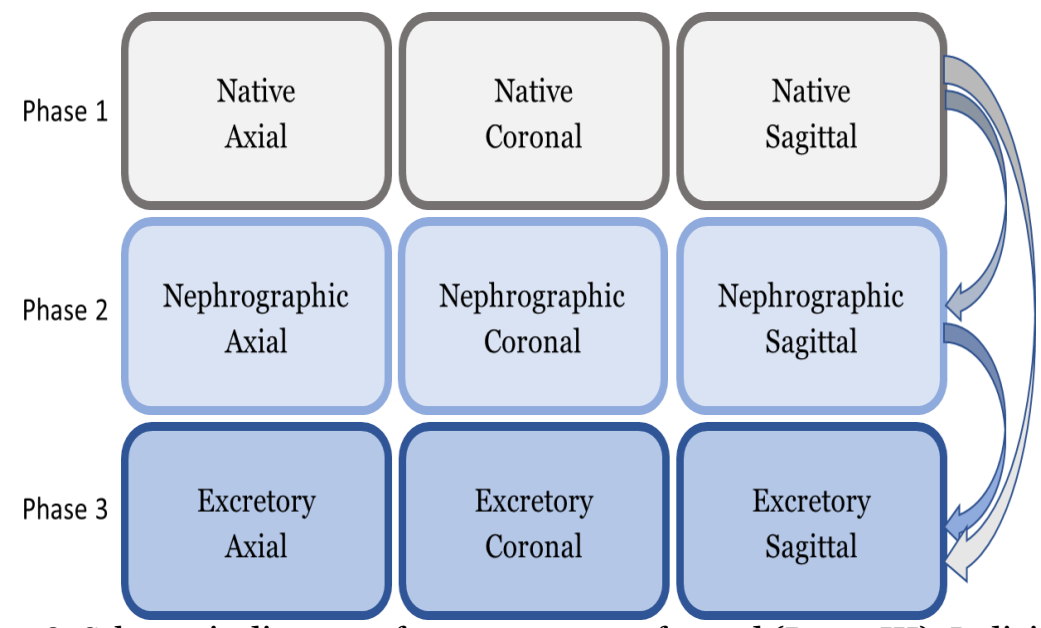

Figure 8. Schematic diagram of assessments performed (Paper III). Individual multi-planar (MPR) phase assessments were performed in a standard dose CTU. In the subsequent statistical analysis, phases 1 and 2 were compared to phase 3 and phase 1 was also compared to phase 2 .

\section{Assessment of image quality}

\section{Visual assessment}

Visual assessment was performed with pairwise comparison of images reconstructed at different tube loads in the same patient for Papers I, II and IV and absolute image quality evaluation in the three phases of a CTU in Paper III. To determine how certain the readers were in their scoring of the image criteria and pathological findings, variation in certainty scores between the three phases were also calculated by grouping the score options 1 and 5 (normal/pathological examination) for high certainty, 2 and 4 (probably normal/pathological examination) for medium certainty, and 3 (indecisive) for low certainty. The image criteria with the grading scores used for each paper are summarised in Table 9 and Table 10. 


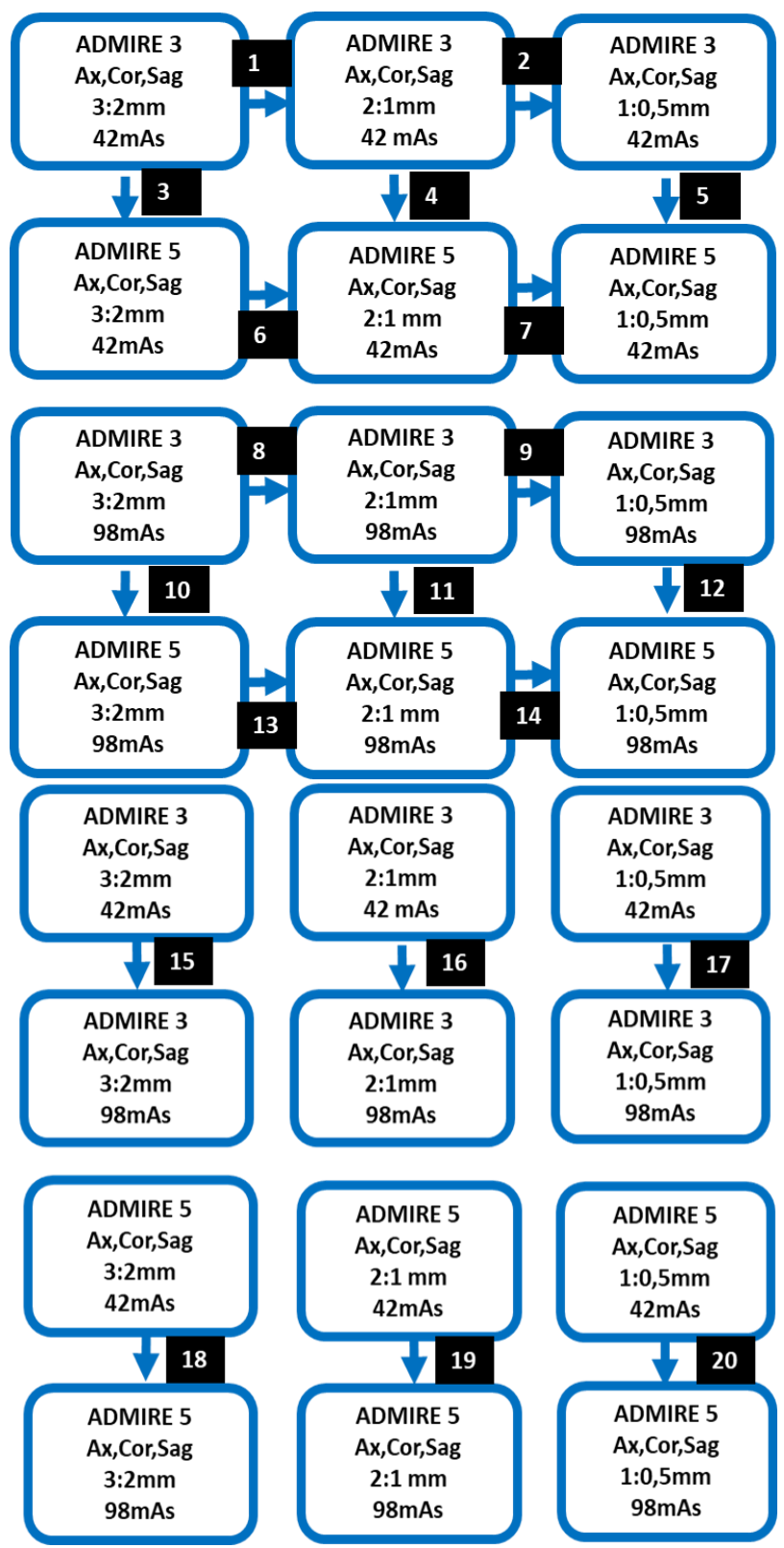

Figure 9. Schematic diagram of multi-planar pairwise comparisons performed in a standard dose abdominal CT (Paper IV). Two tube loads were reconstructed with Advanced modeled iterative reconstruction (ADMIRE) strengths 3 and 5 and 3 slice thicknesses. The arrows show which comparisons were performed. 
Table 9. Overview of image criteria assessed for each study. In Paper I: low-dose abdominal CT, Paper II \& IV: standard-dose abdominal CT and Paper III: CT Urography.

\begin{tabular}{|c|c|}
\hline & Image criteria \\
\hline Paper I & $\begin{array}{l}\text { C1 Visually sharp reproduction of intestine } \\
\text { C2 Visually sharp reproduction of pancreatic contours } \\
\text { C3 Visually sharp reproduction of kidneys \& proximal ureters } \\
\text { C4 Visually sharp reproduction of the aorta } \\
\text { C5 Critical reproduction of the gallbladder wall } \\
\text { C6 Visualisation of pathology }\end{array}$ \\
\hline $\begin{array}{c}\text { Paper } \\
\text { II }\end{array}$ & $\begin{array}{l}\text { C1 Visually sharp reproduction of liver parenchyma } \\
\text { C2 Visually sharp reproduction of pancreas contour } \\
\text { C3 Visually sharp reproduction of kidneys \& proximal ureters } \\
\text { C4 Reproduction of contours of lymph nodes }<15 \mathrm{~mm} \text { in diameter } \\
\text { C5 Image noise not affecting interpretation } \\
\text { C6 Overall image quality for diagnostic purposes }\end{array}$ \\
\hline $\begin{array}{c}\text { Paper } \\
\text { III }\end{array}$ & $\begin{array}{l}\text { C1 Visually sharp reproduction of renal parenchyma } \\
\text { C2 Visually sharp reproduction of renal pelvis \& calyces } \\
\text { C3 Visually sharp reproduction of proximal part of the ureters } \\
\text { C4 Visually sharp reproduction of the renal arteries } \\
\text { C5 Pathology in kidneys \& urinary tract related to abdominal symptoms } \\
\text { C6 Other pathology related to abdominal symptoms } \\
\text { C7 Incidental findings without clinical significance }\end{array}$ \\
\hline $\begin{array}{c}\text { Paper } \\
\text { IV }\end{array}$ & $\begin{array}{l}\text { C1 Visually sharp reproduction of liver parenchyma } \\
\text { C2 Visually sharp reproduction of pancreas contour } \\
\text { C3 Visually sharp reproduction of kidneys \& proximal ureters } \\
\text { C4 Reproduction of contours of lymph nodes }<15 \mathrm{~mm} \text { in diameter } \\
\text { C5 Overall image quality for diagnostic purposes }\end{array}$ \\
\hline
\end{tabular}


Table 10. Overview of grading scales used for the assessment of image criteria and pathological findings in Papers I-IV.

\begin{tabular}{|c|c|}
\hline \multirow[b]{2}{*}{$\begin{array}{c}\text { Papers } \\
\text { I } \\
\text { II } \\
\text { IV }\end{array}$} & Grading scales for image criteria \\
\hline & $\begin{array}{l}\text {-2 Images on left monitor are better than images on right monitor } \\
-1 \text { Images on left monitor are probably better than images on right monitor } \\
0 \text { Images on left and right monitor are equivalent } \\
+1 \text { Images on right monitor are probably better than images on left monitor } \\
+2 \text { Images on right monitor are better than images on left monitor }\end{array}$ \\
\hline \multirow{3}{*}{$\begin{array}{l}\text { Paper } \\
\text { III }\end{array}$} & $\begin{array}{l}\text { Grading scales for image criteria } \\
\text { 1. Criterion was fulfilled } \\
\text { 2. Criterion was probably fulfilled } \\
\text { 3. Indecisive } \\
\text { 4. Criterion was probably not fulfilled } \\
\text { 5. Criterion was not fulfilled }\end{array}$ \\
\hline & Grading scales for pathological findings \\
\hline & $\begin{array}{l}\text { 1: Normal examination } \\
\text { 2: Probably normal examination } \\
\text { 3: Inconclusive examination } \\
\text { 4: Probably pathological examination } \\
\text { 5: Pathological examination }\end{array}$ \\
\hline
\end{tabular}

\section{Objective assessment}

In paper II, scans using a CTDI phantom were performed to assess if the actual radiation dose distribution between the two x-ray sources was in compliance with the tube loads selected for the experimental set up.

In Paper IV, quantitative measurements were performed in phantoms imaged in the same CT-scanner and with the same acquisition parameters as the patients included in the study on images reconstructed with AD3, AD5 and FBP as a baseline for comparison. Standard deviation in HU (SD) contrast-to-noise ratio (CNR), and noise-power spectra (NPS) were measured in images of an anthropomorphic abdominal phantom with an extension ring (QRM.de, Quality Assurance in Radiology and Medicine GmbH). 


\section{Standard deviation (SD) and Contrast-to-Noise Ratio (CNR)}

The SD was measured in $32^{2}$ ROI pixels in the liver.

The CNR was derived as the absolute difference between average Hounsfield Units (HU-values) in the liver ROI and the adjacent background material outside the liver ROI divided by the standard deviation (SD) of the HU-values in background material.

$$
C N R=\left|H U_{\text {liver }}-H U_{\text {background }}\right| / S D_{\text {background }}
$$

SD and CNR were measured in 47,94 and 187 separate images (i) with slice thicknesses of $3 \mathrm{~mm}, 2 \mathrm{~mm}$ and $1 \mathrm{~mm}$, respectively.

\section{Noise-Power Spectrum (NPS)}

The 2D NPS was computed using the expression below from Verdun et al. [76].

$$
N P S_{2 D}\left(f_{x}, f_{y}\right)=\frac{\Delta_{x} \Delta_{y}}{L_{x} L_{y}} \frac{1}{N_{R O I}} \sum_{i=1}^{N_{R O I}}\left|F T_{2 D}\left\{R O I_{i}(x, y)-\overline{R O I_{i}}\right\}\right|^{2}
$$

where $f_{\mathrm{x}}$ and $f_{\mathrm{y}}$ are the spatial frequencies in the $\mathrm{x}$ and $\mathrm{y}$ directions in the 2D NPS. $\Delta_{x}$ and $\Delta_{y}$ both are equal to pixel size of $0.85 \mathrm{~mm}$ and $L_{\mathrm{x}}$ and $L_{\mathrm{y}}$ are the number of pixels along the $\mathrm{x}$ - and y-axis, both equal to $64 . N_{R O I}$ is the number of ROIs used in the average operation, $R O I_{i}(x, y)$ is the region of interest in the phantom liver in the $i$-th slice and $\overline{R O I_{i}}$ the average HUvalue in $R O I_{i}$. The radial 1-dimensional NPS was computed from the average of the $i$ number of 2D NPS, as a function of spatial frequency $(f)$

$$
f=\sqrt{f_{x}^{2}+f_{y}^{2}}
$$

Due to the fewer number of samples in the 2D NPS close to the origin, the precision is reduced at low spatial frequencies.

\section{Statistical analysis}

Image quality scores were statistically analysed using visual grading regression (VGR) [55]. Statistical analyses were performed with the software Stata 13.1 (Stata Corporation LP, College Station, TX, USA) using multilevel mixed effects ordered logistic regression (the meologit command or, in Paper I, its predecessor Generalised Linear and Latent Mixed models 
(GLLAM). The regression coefficients describe how the image quality depends on the choice of tube load and reconstruction algorithm, respectively. By relating two of these coefficients to each other, it was possible to estimate the potential dose reduction using equation 14 [68] (Papers I, II and IV).

For paper III, meologit was used and the regression coefficients describe the combined effect of dose and intravenous contrast on image quality, related to each of the phases of the CTU. The certainty scores were analysed using the same statistical model as the original scores.

The statistical regression models used for each of the papers are illustrated in Figures 10 to 13. Nominal variables are denoted by N, ordinal variables by $\mathrm{O}$ and interval variables by I. Variables representing patient and observer identity, which are not of interest in themselves but are included only to account for the variability within the corresponding two populations, are treated as random effects and denoted by the green $\mathrm{N}$ surrounded by a dashed square. Fixed effects in the models are represented by the variables representing tube loads, algorithms, phases and slice thickness.

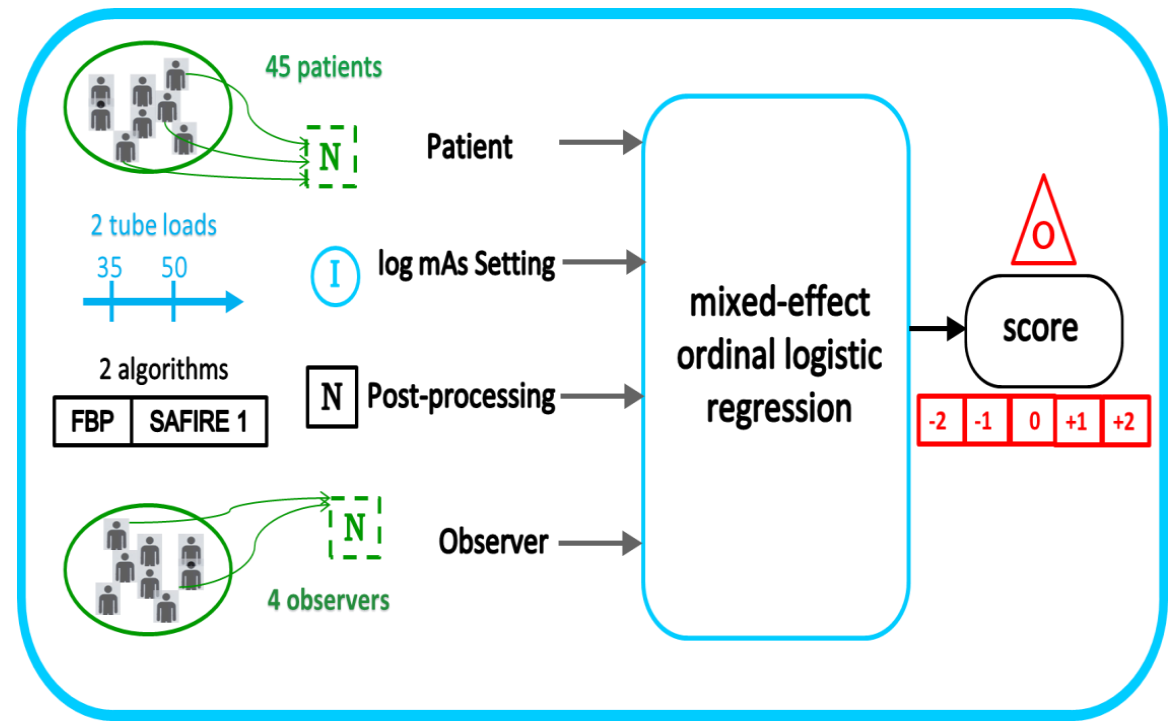

Figure 10. Statistical model for Paper I: Visual grading regression model assessing the effect of 2 tube loads and 2 algorithms in a low-dose abdominal CT with pairwise comparison of images from 45 patients evaluated by 4 independent observers.

In Papers I, II, and IV observer agreement was assessed as a percentage agreement and with the weighted kappa coefficient $\left(\kappa_{w}\right)$. 


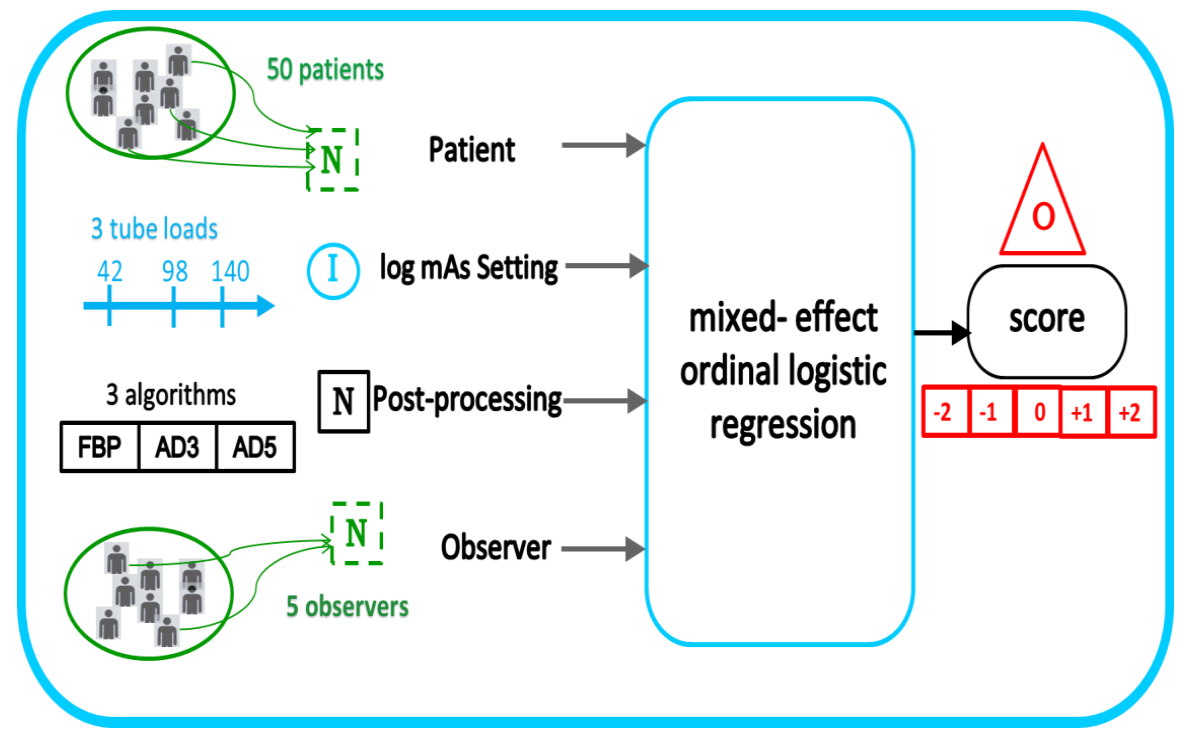

Figure 11. Statistical model for Paper II: Visual grading regression model assessing the effect of 3 tube loads and 3 algorithms in a standard dose abdominal CT with pairwise comparison of images from 50 patients evaluated by 5 independent observers.

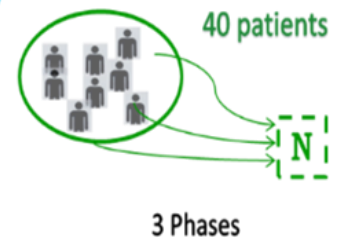

\begin{tabular}{|l|l|l|}
\hline Native & Nephrogram & Excretory \\
\hline
\end{tabular}

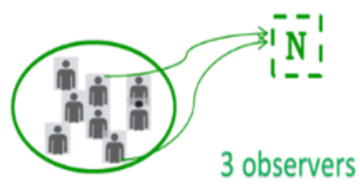

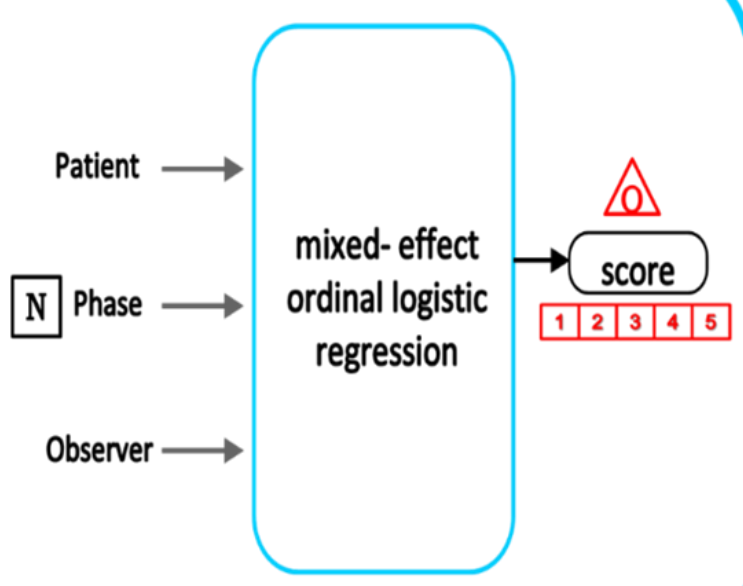

Figure 12. Statistical model for Paper III: Visual grading regression model assessing the effect of 3 phases of a CTU from 40 patients with each individual phase evaluated separately by 3 independent observers. 


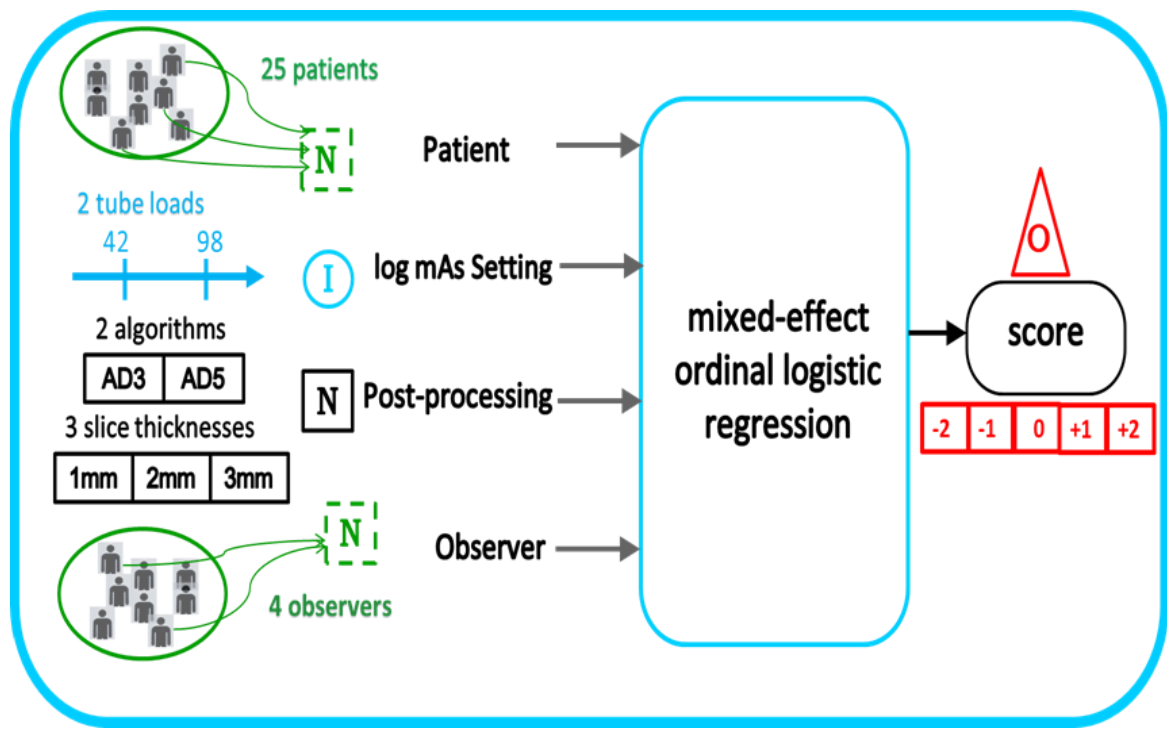

Figure 13. Statistical model for Paper IV: Visual grading regression model assessing the effect of 2 tube loads, 2 algorithms and 3 slice thicknesses in a standard dose abdominal CT with pairwise comparison of images from 25 patients evaluated by 4 independent observers. 
Visual grading evaluation and optimisation in abdominal Computed Tomography 


\section{RESULTS}

\section{Paper I}

The study evaluated the potential for dose reduction in low dose abdominal CT by comparing images reconstructed with FBP and SAFIRE strength 1 at two tube loads while preserving the image quality (Figure 14).

When comparing the same algorithm at different tube loads, an increase in dose renders better image quality for all criteria assessed shown by the stronger significant effects of $\log (\mathrm{mAs})(p \leq 0.001)$. When comparing FBP and SAFIRE strength 1, improvement in image quality is observed for SAFIRE with a significant effect $(p \leq 0.01)$ and an estimated potential dose reduction of $5-9 \%$ for all image criteria assessed (Table 11).

Table 11. VGR coefficients for all the image criteria with estimated dose reduction values for pairwise comparison of images acquired at $50 \mathrm{mAs}$ and $35 \mathrm{mAs}$ and reconstructed with SAFIRE and Filtered back projection (FBP)

\begin{tabular}{|l|c|c|c|}
\hline \multirow{2}{*}{\multicolumn{1}{|c|}{ Criterion }} & \multicolumn{2}{|c|}{ Regression Coefficients } & \multirow{2}{*}{$\begin{array}{c}\text { Estimated dose } \\
\text { reduction (\%) } \\
(95 \% \text { CI) }\end{array}$} \\
\cline { 2 - 3 } & $\begin{array}{c}\text { log } \\
(\mathbf{m A s})\end{array}$ & $\begin{array}{c}\text { SAFIRE } \\
\text { reconstruction }\end{array}$ & $8(6 ; 11)$ \\
\hline 1. The intestine & $6.95^{* *}$ & $0.61^{* * *}$ & $7(5 ; 9)$ \\
\hline 2. Pancreatic contours & $7.80^{* *}$ & $0.55^{* *}$ & $9(7 ; 11)$ \\
\hline $\begin{array}{l}\text { 3. Kidneys and proximal } \\
\text { ureters }\end{array}$ & $8.56^{* *}$ & $0.79^{* *}$ & $9(7 ; 12)$ \\
\hline 4. The aorta & $9.49^{* *}$ & $0.94^{* *}$ & $5(2 ; 8)$ \\
\hline 5. Gallbladder wall & $6.87^{* *}$ & $0.36^{*}$ & \\
\hline${ }^{* * *} p \leq 0.001,{ }^{*} p \leq 0.01$ & \multicolumn{3}{|c}{} \\
\hline
\end{tabular}

Scores for the assessment of pathology were evenly distributed in all pairwise comparisons with no significant effect of either mAs or reconstruction algorithm. When comparing dose reduction potential between groups with $\mathrm{BMI}<30$ and $\mathrm{BMI}>30$, the dose reduction estimates were slightly higher in the former group. Inter-observer agreement ranged from $70 \%$ to $91 \%$ and $K_{w}$ from -0.01 to 0.57 . 
(a)

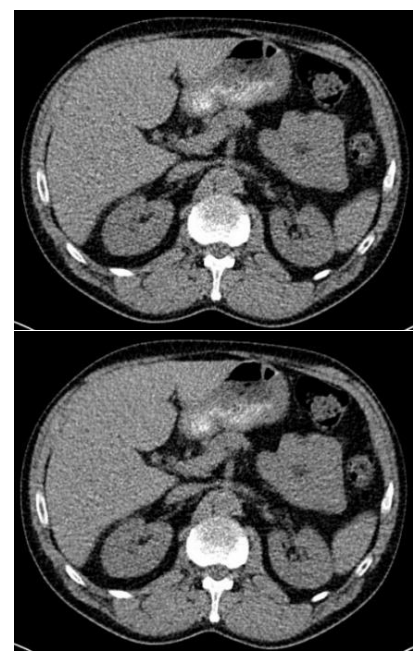

(b)

(d)

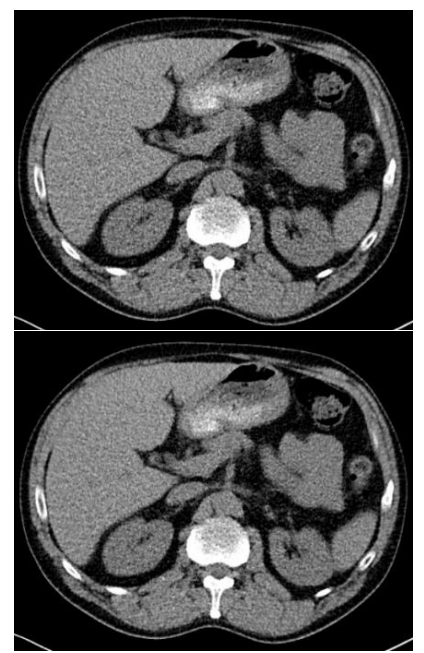

Figure 14. Examples of image comparisons from a study patient, reconstructed with (a) FBP at 35 mAs (b) FBP at 50 mAs (c) SAFIRE strength 1 at $35 \mathrm{mAs}$ and (d) SAFIRE strength 1 at 50 mAs.

$C T$ images may not be adequately reproduced in print.

\section{Paper II}

The study evaluated the dose reduction potential of the iterative ADMIRE algorithm compared to FBP in a standard dose abdominal CT with pairwise comparison of axial images reconstructed at 3 dose-levels (quality reference $\mathrm{mAs}$ (Q ref) 42, 98 and $140 \mathrm{mAs}$ ) and reconstruction algorithms FBP, ADMIRE strengths 3 and 5 (Figure 15). Of the 50 patients included in the study half of the patients underwent contrast-enhanced and the other nonenhanced examinations.

Since no improvement in image quality was seen for comparisons between 98 and $140 \mathrm{mAs}$, a $30 \%$ dose reduction was possible without a change in algorithm strength. A statistical analysis was therefore performed for comparison between 42 and $98 \mathrm{mAs}$ tube loads where improved image quality was observed as a significant strong effect of log tube load and reconstruction method with potential dose reduction relative to FBP of 22-47\% for ADMIRE strength 3 ( $p<0.001)$. For ADMIRE strength 5 , no dose reduction was possible for image criterion 1 (liver parenchyma), but dose reductions between $34-74 \%$ were achieved for other image criteria (Table 12). 
(a)

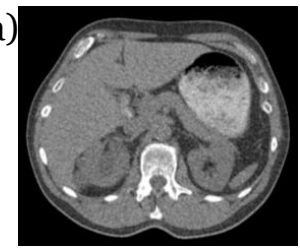

(d)

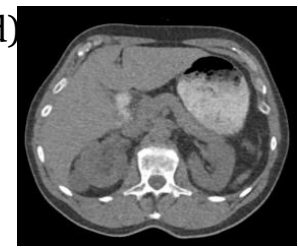

(g)

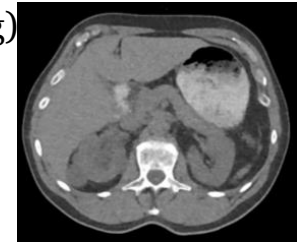

(b)

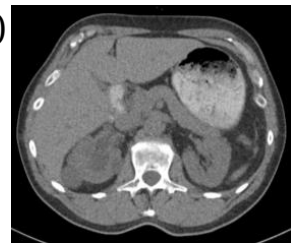

(e)

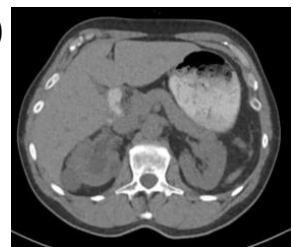

(h)

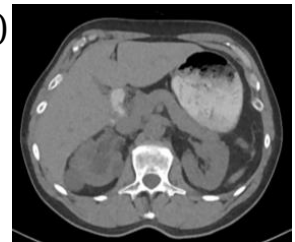

(c)

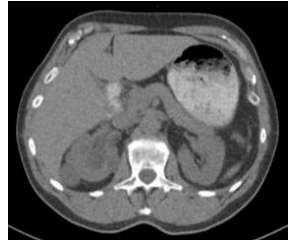

(f)

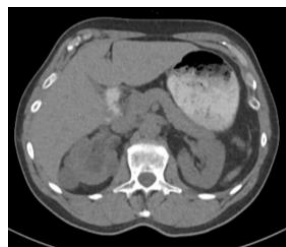

(i)

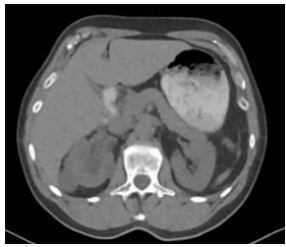

Figure 15. Examples of image comparisons from a study patient, reconstructed with (a) FBP at $42 \mathrm{mAs}$ (b) FBP at $98 \mathrm{mAs}$ (c) FBP at $140 \mathrm{mAs}$ (d) ADMIRE strength 3 at 42 mAs (e) ADMIRE strength 3 at 98 mAs (f) ADMIRE strength 3 at 140 mAs (g) ADMIRE strength 5 at 42 mAs (h) ADMIRE strength 5 at 98 mAs and (i) ADMIRE strength 5 at 140 mAs.

CT images may not be adequately reproduced in print.

Table 12. VGR coefficients for all the image criteria with estimated dose reduction values for pairwise comparison of images acquired at quality reference mAs (Qref) $42 \mathrm{mAs}$ and $98 \mathrm{mAs}$ and reconstructed with Advanced modeled iterative reconstruction (ADMIRE) and Filtered back projection (FBP).

\begin{tabular}{|c|c|c|c|c|c|}
\hline \multirow[t]{2}{*}{ Criterion } & \multicolumn{3}{|c|}{ Regression Coefficients } & \multicolumn{2}{|c|}{$\begin{array}{c}\text { Estimated dose } \\
\text { reductions (\%) } \\
(95 \% \mathrm{CI})\end{array}$} \\
\hline & $\begin{array}{c}\text { Log } \\
(\mathbf{m A s})\end{array}$ & AD3 & AD5 & AD3 & AD5 \\
\hline 1. Liver parenchyma & $2.28^{* * *}$ & $0.57^{* * *}$ & $-0.08^{\circ}$ & $22(11 ; 33)$ & - \\
\hline 2. Pancreatic contours & $2.00^{* * *}$ & $0.92^{* * *}$ & $1.73^{* * *}$ & $37(26 ; 48)$ & $58(53 ; 63)$ \\
\hline $\begin{array}{l}\text { 3. Kidneys and } \\
\text { proximal ureters }\end{array}$ & $2.21^{* * *}$ & $1.11^{* * *}$ & $2.09^{* * *}$ & $40(31 ; 49)$ & $61(57 ; 66)$ \\
\hline $\begin{array}{l}\text { 4. Lymph nodes }<15 \\
\text { mm in diameter }\end{array}$ & $1.72^{* * *}$ & $1.05^{* * *}$ & $1.93^{* * *}$ & $46(35 ; 57)$ & $67(63 ; 72)$ \\
\hline 5. Image noise & $2.38^{* * *}$ & $1.50^{* * *}$ & $3.16^{* * *}$ & $47(39 ; 55)$ & $74(71 ; 76)$ \\
\hline $\begin{array}{l}\text { 6. Overall image } \\
\text { quality }\end{array}$ & $2.69^{* * *}$ & $1.06^{* * *}$ & $1.10^{* * *}$ & $33(24 ; 41)$ & $34(27 ; 40)$ \\
\hline
\end{tabular}




\section{Paper III}

The study evaluated the diagnostic value of a low-dose series by absolute comparison of the three phases of a CTU (Figure 16) to ascertain when the low-dose series was sufficient to reduce the radiation burden for certain patient groups.

Visualisation of renal anatomical criteria was as expected with each post-contrast phase showing better image quality compared to the native phase. Pathology assessment, on the other hand, was not significantly different between the three phases (Table 13). When grouping the criterion scores to determine the certainty of the observers' scoring decision, similar results were observed with improved image quality for the anatomical criteria, in favour of post-contrast phases when compared to the native phase. Also, renal pathology was determined with greater certainty in both postcontrast phases when compared to the native phase as was incidental pathology, which was significant in favour of the nephrographic phase when compared to the native phase (Table 14). When assessing if an examination was normal or pathological, the low number of inconclusive scores indicated that it was possible to distinguish between the two categories.

(a)

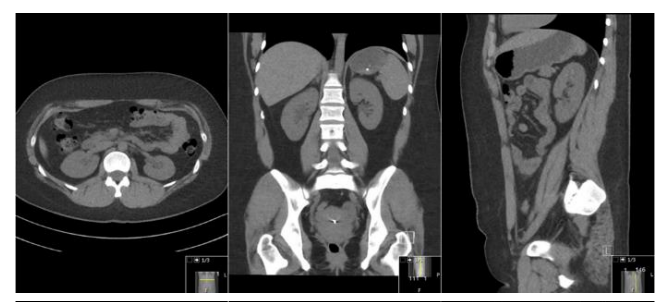

(b)

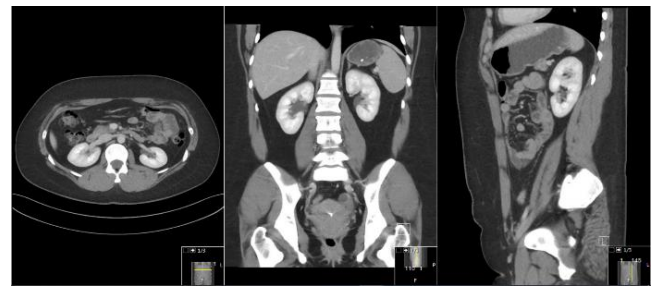

(c)

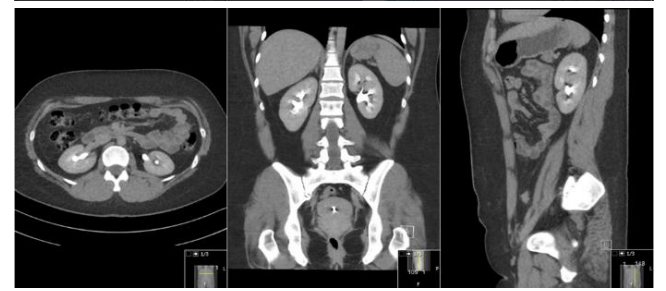

Figure 16. Examples of image comparisons from a study patient. MPR reconstructions of (a) native phase (b) nephrographic phase (c) excretory phase in a CT Urography. CT images may not be adequately reproduced in print. 
Table 13. Significance of score differences between phases tested with mixed-effects ordinal logistic regression and pairwise comparisons using Bonferroni correction.

\begin{tabular}{|c|c|c|c|}
\hline \multirow[b]{2}{*}{ Criterion } & \multicolumn{3}{|c|}{ Phase comparisons } \\
\hline & $\begin{array}{c}\text { Native } \\
\text { vs } \\
\text { Nephrographic } \\
\end{array}$ & $\begin{array}{c}\text { Native } \\
\text { vs } \\
\text { Excretory } \\
\end{array}$ & $\begin{array}{c}\text { Nephrographic } \\
\text { vs } \\
\text { Excretory } \\
\end{array}$ \\
\hline 1. Renal parenchyma & $p<0.001$ & $p<0.001$ & $p<0.001$ \\
\hline 2. Renal pelvis/calyxes & $p<0.001$ & $p<0.001$ & $p<0.001$ \\
\hline 3. Proximal ureters & $p<0.001$ & $p<0.001$ & $p<0.05$ \\
\hline 4. Renal arteries & $p<0.001$ & $p<0.001$ & $p<0.001$ \\
\hline 5. Renal pathology & n.s. & n.s. & n.s. \\
\hline 6. Other abdominal pathology & n.s. & n.s. & n.s. \\
\hline 7. Incidental pathology & n.s. & n.s. & n.s. \\
\hline
\end{tabular}

Table 14. Significance of certainty score differences between phases tested with mixed-effects ordinal logistic regression, with pairwise comparisons using Bonferroni correction.

\begin{tabular}{|c|c|c|c|}
\hline \multirow[b]{2}{*}{ Criterion } & \multicolumn{3}{|c|}{ Phase comparisons } \\
\hline & $\begin{array}{c}\text { Native } \\
\text { vs } \\
\text { Nephrographic } \\
\end{array}$ & $\begin{array}{c}\text { Native } \\
\text { vs } \\
\text { Excretory } \\
\end{array}$ & $\begin{array}{c}\text { Nephrographic } \\
\text { vs } \\
\text { Excretory } \\
\end{array}$ \\
\hline 1. Renal parenchyma & $p<0.001$ & $\boldsymbol{p}<\mathbf{0 . 0 0 1}$ & $p<0.001$ \\
\hline 2. Renal pelvis/calyxes & $p<0.001$ & $p<0.001$ & $p<0.001$ \\
\hline 3. Proximal ureters & $p<0.001$ & $p<0.001$ & $p=0.021$ \\
\hline 4. Renal arteries & $p<0.001$ & n.s. & $p<0.001$ \\
\hline 5. Renal pathology & $p<0.05$ & $p<0.05$ & n.s. \\
\hline 6. Other abdominal pathology & n.s. & n.s. & n.s. \\
\hline 7. Incidental pathology & $p<0.01$ & n.s. & n.s. \\
\hline
\end{tabular}

\section{Paper IV}

The study evaluated the dose reduction potential of the iterative ADMIRE algorithm (strengths 3 and 5 ) in a standard dose abdominal CT with pairwise comparison of MPR images reconstructed at 2 tube loads (quality 
reference (Q ref) $\mathrm{mAs} 42$ and $98 \mathrm{mAs}$ ) and 3 slice thickness and increment settings ( $3 \mathrm{~mm}: 2 \mathrm{~mm}, 2 \mathrm{~mm}: 1 \mathrm{~mm}$ and $1 \mathrm{~mm}: 0.5 \mathrm{~mm}$ ). Examples of patient images are shown in Figure 17.

\section{Visual evaluation}

As expected, image quality improved when slice thickness increased from $1 \mathrm{~mm}$ to $2 \mathrm{~mm}$ or $3 \mathrm{~mm}$ (Table 15), independent of the reconstruction algorithm with possible dose reduction estimates presented in Table 16.

Table 15. Visual Grading Regression (VGR) coefficients for all image criteria assessed in a pairwise comparison of MPR images reconstructed at 2 tube loads with two ADMIRE strengths and three slice thicknesses.

\begin{tabular}{|c|c|c|c|c|c|}
\hline \multirow{3}{*}{ Criterion } & \multicolumn{5}{|c|}{ Regression coefficients } \\
\hline & \multirow{2}{*}{$\begin{array}{c}\text { Log } \\
(\mathrm{mAs})\end{array}$} & \multirow{2}{*}{$\begin{array}{c}\text { AD } 5 \\
\text { vs. } \\
\text { AD } 3\end{array}$} & \multicolumn{3}{|c|}{ Slice thickness (mm) } \\
\hline & & & 2 vs. 1 & 3 vs.1 & 3 vs. 2 \\
\hline 1. Liver parenchyma & $1.25^{* * *}$ & $-1.35^{* * *}$ & $0.49^{* * *}$ & $0.53^{* * *}$ & $0.04^{\circ}$ \\
\hline 2. Pancreatic contours & $1.75^{* * *}$ & $0.05^{\circ}$ & $0.47^{* * *}$ & $0.50^{* * *}$ & $0.03^{\circ}$ \\
\hline $\begin{array}{l}\text { 3. Kidneys and } \\
\text { proximal ureters }\end{array}$ & $1.78^{* * *}$ & $0.21^{* *}$ & $0.55^{* * *}$ & $0.55^{* * *}$ & $0.004^{\circ}$ \\
\hline $\begin{array}{l}\text { 4. Lymph nodes }<15 \mathrm{~mm} \\
\text { in diameter }\end{array}$ & $1.55^{* * *}$ & $0.48^{* * *}$ & $0.51^{* * *}$ & $0.49^{* * *}$ & $-0.02^{\circ}$ \\
\hline 5. Overall image quality & $1.65^{* * *}$ & $-0.86^{* * *}$ & $0.71^{* * *}$ & $0.87^{* * *}$ & $0.16^{\circ}$ \\
\hline
\end{tabular}

The higher strength of the algorithm (ADMIRE 5) compared to ADMIRE 3 has diverse effects on image quality depending on the slice thickness and further dose reductions were limited to certain image criteria (Table 17). 
(a) ADMIRE strength 3 \& 5 at $42-$ and $98 \mathrm{mAs}$ and $1 \mathrm{~mm}$ slice thickness
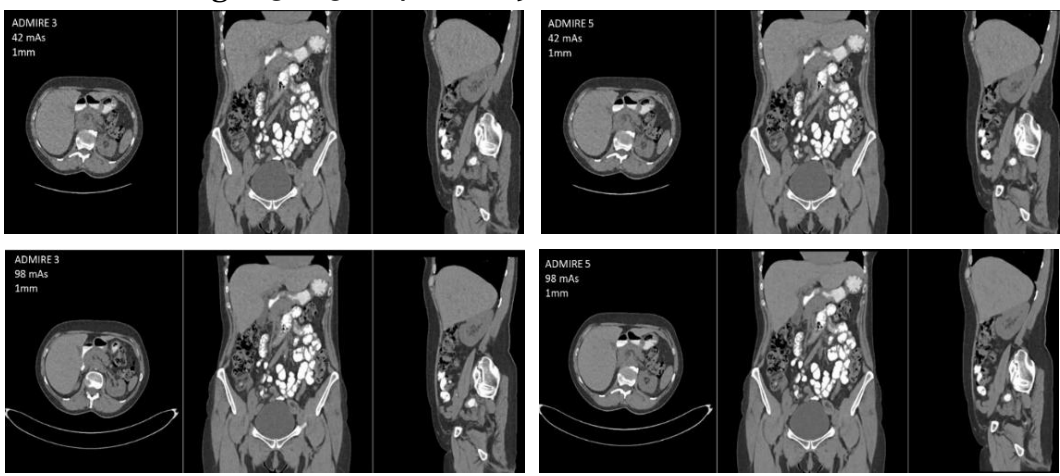

(b) ADMIRE strength $3 \& 5$ at 42- and $98 \mathrm{mAs}$ and $2 \mathrm{~mm}$ slice thickness
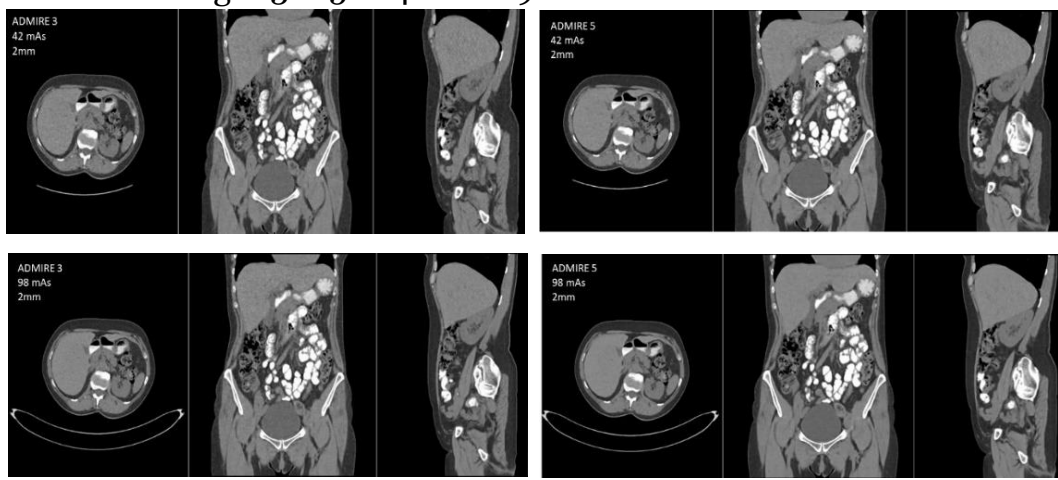

(c) ADMIRE strength $3 \& 5$ at 42- and $98 \mathrm{mAs}$ and $3 \mathrm{~mm}$ slice thickness
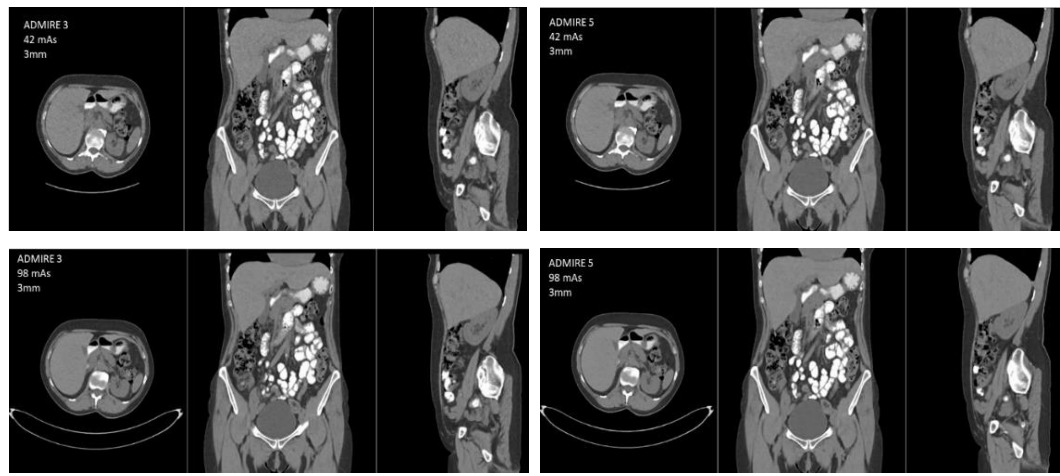

Figure 17. Visual demonstration of CT abdomen images obtained from a study patient at tube loads $42 \mathrm{mAs}$ and $98 \mathrm{mAs}$ and reconstructed with ADMIRE strengths 3 and 5 at slice thickness of (a) $1 \mathrm{~mm}$, (b) $2 \mathrm{~mm}$ and (c) $3 \mathrm{~mm}$. CT images may not be adequately reproduced in print. 
Table 16. Estimates of potential dose reductions for variation in slice thickness from $1 \mathrm{~mm}$ to $2 \mathrm{~mm}$ and $1 \mathrm{~mm}$ to $3 \mathrm{~mm}$ independent of the ADMIRE algorithm strength.

\begin{tabular}{|l|c|c|}
\hline \multirow{2}{*}{\multicolumn{1}{|c|}{ Criterion }} & \multicolumn{2}{c|}{$\begin{array}{c}\text { Estimated dose reduction (\%) } \\
\text { (95\% confidence limits) }\end{array}$} \\
\cline { 2 - 3 } & $\begin{array}{c}\text { Slice thickness } \\
\text { 2 mm vs. 1 mm }\end{array}$ & $\begin{array}{c}\text { Slice thickness } \\
\text { 3 mm vs. 1 mm }\end{array}$ \\
\hline 1. Liver parenchyma & $32(22 ; 43)$ & $35(21 ; 48)$ \\
\hline 2. Pancreatic contours & $24(16 ; 32)$ & $25(15 ; 35)$ \\
\hline 3. Kidneys and proximal ureters & $26(19 ; 34)$ & $27(16 ; 37)$ \\
\hline $\begin{array}{l}\text { 4. Lymph nodes < 15 mm in } \\
\text { diameter }\end{array}$ & $28(19 ; 37)$ & $27(15 ; 39)$ \\
\hline 5. Overall image quality & $35(27 ; 43)$ & $41(31 ; 50)$ \\
\hline
\end{tabular}

Table 17. Visual Grading Regression (VGR) coefficients for all image criteria for comparison of images reconstructed at 2 tube loads and with 2 reconstruction algorithms, analysed separately for comparisons involving only one slice thickness.

\begin{tabular}{|c|c|c|c|c|c|c|}
\hline \multirow{3}{*}{ Criterion } & \multicolumn{6}{|c|}{ Regression coefficients } \\
\hline & \multicolumn{2}{|c|}{$\begin{array}{l}\text { Comparison } \\
\text { 1mm slices }\end{array}$} & \multicolumn{2}{|c|}{$\begin{array}{l}\text { Comparison } \\
\text { 2mm slices }\end{array}$} & \multicolumn{2}{|c|}{$\begin{array}{l}\text { Comparison } \\
\text { 3mm slices }\end{array}$} \\
\hline & $\begin{array}{c}\log \\
(\mathbf{m A s})\end{array}$ & \begin{tabular}{|c|} 
AD5 \\
vs. \\
AD3 \\
\end{tabular} & $\begin{array}{c}\log \\
(\mathrm{mAs})\end{array}$ & \begin{tabular}{|c|} 
AD5 \\
vs. \\
AD3 \\
\end{tabular} & $\begin{array}{c}\log \\
(\mathrm{mAs})\end{array}$ & $\begin{array}{c}\text { AD5 } \\
\text { vs. } \\
\text { AD3 }\end{array}$ \\
\hline 1. Liver parenchyma & $2.06^{* * *}$ & $-0.55^{* * *}$ & $0.93^{* * *}$ & $-1.35^{* * *}$ & $0.51^{* *}$ & $-1.82^{* * *}$ \\
\hline $\begin{array}{l}\text { 2. Pancreatic } \\
\text { contours }\end{array}$ & $2.39^{* * *}$ & $0.54^{* * *}$ & $1.35^{* * *}$ & $-0.06^{\circ}$ & $0.87^{* * *}$ & $-0.26^{*}$ \\
\hline $\begin{array}{l}\text { 3. Kidneys and } \\
\text { proximal ureters }\end{array}$ & $2.47^{* * *}$ & $0.63^{* * *}$ & $1.25^{* * *}$ & $0.13^{\circ}$ & $0.93^{* * *}$ & $-0.16^{\circ}$ \\
\hline $\begin{array}{l}\text { 4. Lymph nodes } \\
<15 \mathrm{~mm} \text { in } \\
\text { diameter }\end{array}$ & $2.22^{* * *}$ & $0.87^{* * *}$ & $1.24^{* * *}$ & $0.39^{* *}$ & $0.72^{* * *}$ & $0.00^{\circ}$ \\
\hline $\begin{array}{l}\text { 5. Overall image } \\
\text { quality }\end{array}$ & $2.38^{* * *}$ & $-0.26^{*}$ & $0.93^{* * *}$ & $-1.35^{* * *}$ & $0.94^{* * *}$ & $-1.12^{* * *}$ \\
\hline
\end{tabular}

\section{Objective evaluation}

With an increase in tube load and iterative algorithm strength compared to FBP, noise (SD) decreases while the CNR increases (Table 18). 
Table 18. Effect of tube load (Qref. mAs), reconstruction algorithms ADMIRE strength 3 (AD3), strength 5 (AD5), Filtered back projection (FBP) and slice thickness on noise estimated as standard deviation (SD) and contrast-to-noise ratio (CNR).

\begin{tabular}{|c|c|c|c|c|c|c|c|}
\hline \multirow{2}{*}{$\begin{array}{c}\text { Slice } \\
\text { thickness }\end{array}$} & \multirow{2}{*}{$\begin{array}{l}\text { Qref. } \\
\text { mAs }\end{array}$} & \multicolumn{3}{|c|}{ SD (HU) } & \multicolumn{3}{|c|}{ CNR } \\
\hline & & FBP & AD3 & AD5 & FBP & AD3 & AD5 \\
\hline $3 \mathrm{~mm}$ & 42 & 15.00 & 11.00 & 7.70 & 9.75 & 14.75 & 16.12 \\
\hline $3 \mathrm{~mm}$ & 98 & 12.50 & 9.15 & 6.25 & 12.08 & 16.70 & 22.74 \\
\hline $3 \mathbf{~ m m}$ & 140 & 9.95 & 7.20 & 5.05 & 16.28 & 21.99 & 25.71 \\
\hline $2 \mathrm{~mm}$ & 42 & 19.00 & 14.00 & 10.50 & 7.85 & 10.46 & 14.49 \\
\hline $2 \mathrm{~mm}$ & 98 & 16.00 & 12.00 & 8.60 & 9.33 & 12.86 & 18.59 \\
\hline 2 mm & 140 & 13.00 & 9.50 & 6.80 & 11.81 & 16.94 & 25.23 \\
\hline 1 mm & 42 & 25.50 & 19.50 & 14.00 & 5.77 & 7.57 & 10.69 \\
\hline $1 \mathrm{~mm}$ & 98 & 22.00 & 17.00 & 12.00 & 6.28 & 8.78 & 11.81 \\
\hline $1 \mathbf{~ m m}$ & 140 & 17.00 & 13.00 & 9.45 & 10.20 & 13.11 & 18.25 \\
\hline
\end{tabular}

Confidence intervals related to the SD and CNR measurements are presented as error bars in Figures 4 and 5 (Paper IV).

The noise-power decreases with increasing ADMIRE strength compared to FBP and is more pronounced at the higher spatial frequencies when using ADMIRE 3, and particularly with ADMIRE 5, compared to FBP. The dotted vertical lines indicate the median spatial frequency values (Figure 18)

As expected, the noise power decreases with increasing tube load and slice thickness as shown for ADMIRE 3 for slice thickness $1 \mathrm{~mm}, 2 \mathrm{~mm}$ and $3 \mathrm{~mm}$ (Figure 19). 


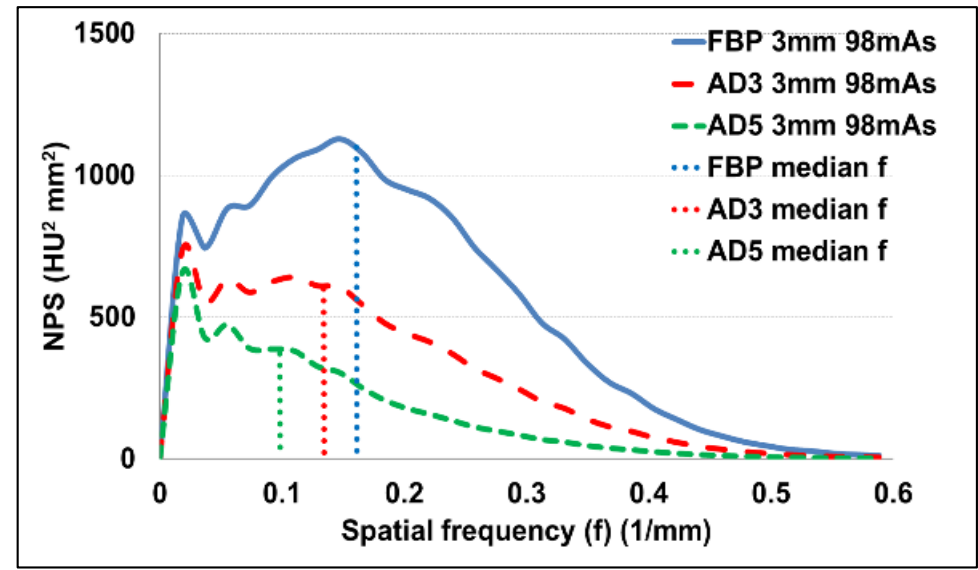

Figure 18. Comparison between ADMIRE strength 3 (AD3), strength 5 (AD5) and Filtered back projection (FBP) at $98 \mathrm{mAs}$ and $3 \mathrm{~mm}$ slice thickness. The vertical dotted lines indicate the median spatial frequency (f) values.

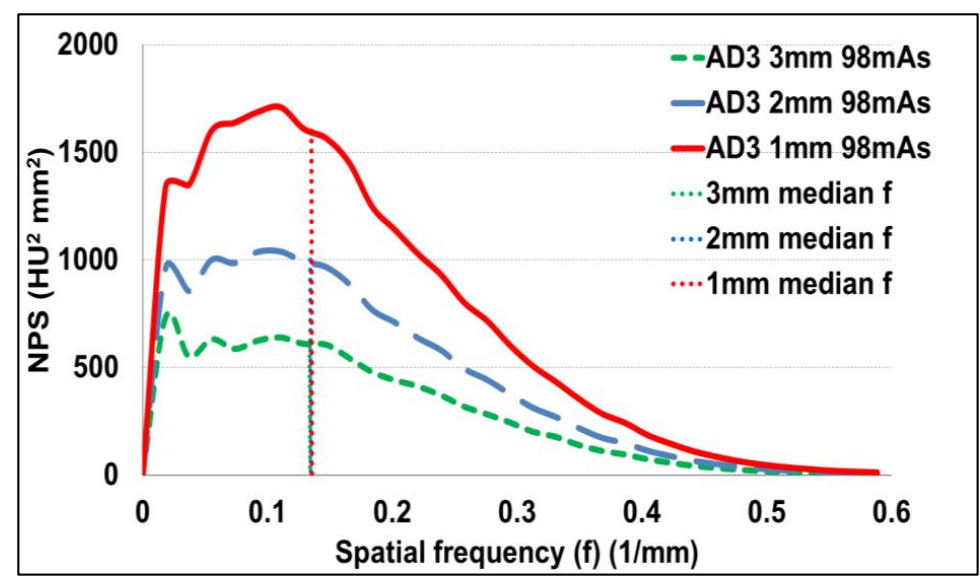

Figure 19. Effect of reconstruction algorithm and slice thickness on noise power spectrum (NPS). Comparison between three slice thicknesses for ADMIRE strength 3 (AD3) at 98 mAs. The vertical dotted lines indicate the median spatial frequency (f) values. 


\section{DISCUSSION}

The evolution and availability of new technology has led to extensive use of diagnostic imaging over the past decade [77, 78]. Changes in health care practices such as fast-tracking of emergency department patients, availability of hospital beds, the introduction of standardised care pathways for cancer patients in 2015 [79] and further development of such in other diseases during 2019 to 2020 [80], have to some extent and will in the future, also contribute to the increase in use of high-tech modalities such as CT and Magnetic resonance imaging (MRI). CT is a patient friendly modality with fast acquisition times and high diagnostic reliability in applicable areas of the diagnostic chain. The increase in the number of CT imaging procedures is also attributed to new applications being made available with the expanding new technology [39, 77]. However, concern has been raised as to the adverse effects of low dose ionising radiation, of which CT is the highest contributor [78]. Multiphase examinations are more common in abdominal CT, and approximately $30 \%$ of CT examinations are abdominal and pelvic, which deliver an effective dose of approximately 6 to $8 \mathrm{mSv}$ [17]. This associated high radiation dose from CT has been a topic vastly debated in the medical community with no consensus reached as to whether the radiation delivered by CT has any carcinogenic effect on the patient population. The LNT model has been a subject of discussion but is still considered as an adequate safety measure for radiation protection purposes, its pros and cons are further discussed in a separate section below [30]. According to the ALARA principle, the optimal dose is that which allows for a reliable diagnosis. As "over reduction" of dose is likely to compromise the high diagnostic accuracy and given the benefits of CT, optimisation is therefore required to abide by the ALARA and AHARA principles [22].

The focus of this thesis was to evaluate the effect of advanced reconstruction methods on image quality and radiation dose reduction in abdominal CT (Papers I, II and IV), as well as the diagnostic value of lowdose abdominal CT (Paper III). In Papers I and II, image quality in FBP was used as a baseline and compared to different strengths of a statistical iterative algorithm (SAFIRE) and a model based iterative algorithm (ADMIRE), respectively. In Paper IV, varying slice thicknesses of two strengths of ADMIRE ( 3 and 5) were compared with each other, where improvement in image quality was limited to certain image criteria for the higher strength of the algorithm. Paper III was an absolute evaluation study of image quality and pathology between the three phases of CTU to determine when the low-dose series was sufficient. 


\section{Radiation risks \& LNT}

The LNT model has been the subject of debate, with doubts raised concerning the existence of scientific evidence showing that low dose radiation is carcinogenic [81]. There are several reports that are based on collective effective dose regarding potential cancer risks and deaths due to exposure to medical imaging. These have also been questioned as the assessments are based on assumptions and therefore undoubtfully are subject to substantial uncertainty [82]. Since the incidence rate of cancer in the general population is quite high (UK 1:2, USA 1:3, Sweden 1:3) [83, 84, 85], if there was any increase in the number of cancers it would be extremely hard to epidemiologically prove the individual small risks are a result of diagnostic x-ray exposure, as very large study populations are required to conduct such epidemiological studies.

The National Council on Radiation Protection (NCRP) reviewed recently conducted epidemiological studies to ascertain if there was support for the LNT model or, to the contrary, if there was evidence to prove that the LNT is not appropriate as used in radiation protection today [32, 33]. The review found support for the LNT model in many, but not all studies of solid cancers and leukaemia, concluding that currently there is no alternative to the practical dose-response relationship provided by the LNT for radiation protection purposes (32).

The International Commission on Radiological Protection (ICRP) state that the use of the collective effective dose as an epidemiological tool to estimate projected risks is inappropriate and instead, mean absorbed organ and tissue doses should be correlated to radiation-induced health effects [30]. There are some hypothetical risks involved with the increasing number of examinations performed leading to higher cumulative population doses. Given the clear benefits derived from use of $x$-rays in medical imaging, some kind of theoretical foundation (such as the LNT) is necessary to support adequate safety measures for radiation protection [30].

\section{Method discussion}

With the mathematical and technical background presented in this thesis, the obvious choice was to perform visual grading experiments to study the effect of iterative reconstruction algorithms on clinical image quality in low-dose (Paper I) and standard dose CT abdomen (Papers II and IV) and ascertain the diagnostic quality of low-dose CT abdomen (Paper III).

When SAFIRE was first introduced clinically, there was a reluctance, among radiologists, to use higher strengths of the algorithm due to the unusual appearance of the images as a result of noise reduction. As SAFIRE strength 1 rendered similar image characteristics to FBP, it was the clinical 
standard used at that time. The study design in Paper I was simple with 5 pairwise comparisons of axial images per patient. The study concluded that a small dose reduction was possible in a low-dose abdominal CT using SAFIRE strength 1. It also indicated the need for further research with higher strengths of the SAFIRE algorithm.

In light of conclusions from Paper I, in Paper II, the intention was to continue to evaluate the algorithm at higher strengths. However, technical advancements and the installation of new equipment (Somatom FORCE dual source CT scanner, Siemens, Erlangen, Germany) presented new opportunities to evaluate the model-based algorithm ADMIRE. This led to a change in the research plan and evaluation of potential dose reduction using ADMIRE. The clinical standard at the time was ADMIRE strength 3; Paper II evaluated the dose reduction potential of strengths 3 and 5 compared to FBP, to ascertain if further dose reduction was possible when using a higher strength of the algorithm in a standard-dose abdominal CT. Paper II concluded that dose reduction was possible for ADMIRE 3. However, for images reconstructed with ADMIRE 5, some assessed criteria were not quite accepted by the radiologists as their diagnostic confidence was affected. It was also noted that the study would have benefited if objective evaluation of image quality had been included to explain the unexpected and interesting results when no improvement in image quality was seen for comparisons between Qref $98 \mathrm{mAs}$ and $140 \mathrm{mAs}$. Since the Somatom Force detectors are more dose-efficient, it is possible that Qref $98 \mathrm{mAs}$ was sufficient enough to produce the highest image quality [86]. As subjective and objective analyses both have different strengths and weakness, a combination of both evaluations would have increased the reliability of the results [51].

It is known that radiologists prefer a certain amount of noise to accentuate the sharpness in the image, which results in increased diagnostic confidence [87]. We hypothesised that if more noise could be introduced (e.g. by reducing slice thickness) to counteract the smoothing effect of ADMIRE, there was a possibility that higher strengths of the algorithm could be accepted in clinical practice. As direct comparisons accentuate the subtle differences between images, pairwise MPR comparisons (which replicate the typical clinical setting), may influence the clinical acceptable image quality. It was therefore of interest to study if further dose reductions were possible with a combination of ADMIRE and variation in image slice thickness together with post-processing methods such as MPR. As such Paper IV evaluated the dose reduction potential when comparing strengths 3 and 5 of the algorithm at varying slice thicknesses in MPR format. Objective image quality evaluation was also included in this paper to complement the subjective evaluation as both methods have their strengths and weakness. The main strength of the objective evaluation method is related to consistency 
and reproducibility, which, in subjective assessment, is the main disadvantage. The disadvantage of objective methods is that they do not assess human perception of images and the variation in observer bias. Nevertheless, subjective evaluation is also prone to various observer biases [51]. Thus, more reliable results could be attained using a combination of both objective and subjective assessments.

There were many pairwise combinations available for comparison when designing the studies in Paper II and IV. However, since all comparisons could not be performed, we debated back and forth as to which pairs would be most appropriate. In Paper II we performed diagonal comparisons to show how image quality differs at different tube loads since data cannot be extrapolated to ascertain image quality differences between pairs that were not compared. In Paper II comparison number 3 should have been made between FBP at 98 mAs and ADMIRE 3 at $42 \mathrm{mAs}$, for consistency. However, this was not apparent when planning the study. In Paper IV, it was assumed that comparisons between tube loads for the same algorithm and same dose for the two algorithms would be sufficient to evaluate the dose reduction potential and simplify the presentation of the results. However, cross-comparisons between the clinical standard and the higher strength at varying slice thickness would have provided valuable information. When planning a study, it is relevant to consider which comparisons are essential in relation to the research questions.

Thus, a transition in study design, from simple in Paper I to increasing complexity in Paper II and Paper IV, was necessary to answer the research questions raised by conclusions of previous papers.

In Paper III, another aspect of optimisation was studied i.e. choice of appropriate imaging technique for the diagnostic task at hand. Since there is a visible difference in the three phases of the CTU protocol, absolute MPR comparisons of each phase were assessed separately to avoid issues such as observer bias which would have been apparent if pairwise comparisons had been used. An absolute grading scale was used to allow for assignment of the same grade to images with similar image quality, thus reducing the risk for observer bias.

\section{Statistical method}

The statistical method (VGR) used in this thesis allowed us to a analyse several parameters simultaneously in all papers, as well as to estimate the potential dose reduction in Papers I, II and IV. VGR is the only statistical method available that produces estimates of potential dose reduction and can be used to conduct pilot studies to evaluate new equipment or reconstruction methods [68]. The basic assumption in visual grading experiments is that pathology detection is correlated to reproduction of anatomy. 
Some special cases have shown that visual grading methods agree not only with detection studies using human observers, but also to advanced calculations of physical image quality. This is an important observation as it validates the above assumption [52].

\section{Bias}

Subjective evaluation studies are prone to observer bias. Pairwise comparison of images involving simultaneous viewing of two images in VGR, intends to increase the sensitivity to small differences in image quality, which may not be possible when viewing the images separately [55]. However some observer bias cannot be avoided as the observers are accustomed to a certain image appearance and noise texture, with preference for the noisy and familiar appearance contra unusual appearance of the image [51].

Despite randomisation of the images for the reading sessions, it was impossible to completely blind the observers as to the differences in image texture and variation in slice numbers, hence some observer bias may have occurred in assessments for Papers I, II and IV. Observer preference for the noisy more familiar image is perhaps not easy to eradicate but previous experience in working with the IR algorithms has shown that accustomisation to the unusual appearances of the images is possible through gradual widening of the observers' "comfort zones". As we experienced with implementation of IR in clinical practice, strength 1 of the algorithm was the preference of choice as it produced images of similar appearance to that of FBP. However, since then, the transition from SAFIRE strength 1 to strength 3 was possible by gradually increasing the strength. The change to ADMIRE strength 3, which is the present standard in clinical practice, has also been painless. It is possible that with time, radiologists can learn to adapt to the image quality with higher strengths of the algorithm and increase their diagnostic confidence. As one of our observers pointed out after the completion of readings for Paper IV, that had we had repeated the readings then, ADMIRE 5 may have received better performance results. This adaptation could be observed with a low-dose abdominal protocol at one of our sites in Östergötland, Sweden, when the use of a low-dose abdominal protocol intended for kidney stone evaluation was extended to include other diagnostics related to the acute abdomen [88].

To compensate for diversity between observers, 3-5 observers were recruited for each of the studies included in this thesis. For Papers II, III and IV appropriate training was provided for the observers in the form of coaching sessions to achieve a similar understanding of the evaluation of the image criteria. 
Confounding factors such as anatomical and pathological diversity were avoided as comparisons were performed using images at different tube loads in the same patient for all papers included in this thesis.

\section{Ethical considerations}

Dose reduction studies preferably require two or more data sets, at different tube loads in the same patient, in order to estimate potential dose reduction. In paper I, two acquisitions per patient were performed to estimate the potential dose reduction and hence, inclusion criteria were defined to avoid any ethical issues.

In paper II, this ethical dilemma was resolved by obtaining a license from Siemens Healthineers for an experimental setup on the dual-source scanner. This allowed simultaneous acquisition of 3 datasets at 3 dose-levels in the same patient in one breath hold. This experimental setup provides an interesting opportunity for future dose reduction studies as it avoids repetitive imaging in the same patient in order to obtain data sets at varying dose levels.

\section{Result discussion}

The literature review has shown that both IR algorithms, SAFIRE and ADMIRE, due to their denoising properties, improve image quality in abdominal CT and allow for dose reductions. The results from Papers I, II and IV generally reflect these findings where a potential dose reduction of approximately $10 \%$ for each strength of the iterative algorithms was possible [89].

SAFIRE has been shown to improve image quality, with a potential dose reduction of $50 \%$ at strengths $2-4$ [90] and between $40-60 \%$ for strengths 1-5 [91] in a standard dose abdominal CT. The dose reduction in Paper I was rather small but the potential for dose reduction may be smaller in low-dose abdominal examinations using strength 1 of the algorithm, as strict optimisation has already produced a protocol near the border of clinical acceptability. Greater dose reductions could have been achieved if the methodology had been applied to a standard dose protocol using higher strengths of the algorithm [91].

Similarly, ADMIRE has been shown to reduce noise by $50 \%$ in a standard dose abdominal CT, where the authors suggest that the amount of noise reduction can be translated into reductions in radiation dose [48, 89]. Ellman et al [92] concluded that ADMIRE strength 3 allows for a 30\% dose reduction in contrast structure subgroups. In their study, ADMIRE strength 5 on the other hand, despite significant higher noise reductions, facilitated radiation dose reductions in structures with high and medium 
contrast subgroups. This is in concurrence with results of Papers II and IV. Noise reduction potential of the algorithm cannot be directly transferred to a dose reduction potential which was also demonstrated by Ellman et al [92]. As objective measurements do not necessarily reflect on the value of the clinical subjective image quality assessments, methods other than noise reduction should be applied to evaluate the dose reduction potentials.

Since the non-linear effects of IR lead to smoothing of the anatomical features and change in appearance of the anatomy in the images, some loss of information is observed when applying higher strengths of the algorithm [92, 93]. Low-contrast detectability is compromised when using higher strengths of ADMIRE [94] as was demonstrated by the inferior image quality for criterium "liver parenchyma" in Papers II and IV when comparing the two strengths of the algorithm. Although some improvement in image quality was seen with thinner slices of the higher strength, a general recommendation for ADMIRE 5 in clinical practice was not indicated (Paper IV). However, it is possible that ADMIRE 5 can be used in task-based protocols where sharp visualisation of an anatomical structure of interest is possible.

Several studies presented contradictory results to Papers II and IV, but these studies were either conducted using different acquisition parameters $[48,89]$ or with phantoms [95]. Some acquisition parameters such as lower $\mathrm{kV}$ and contrast enhancement affect image quality which could explain the improvement of image quality in the first two studies. There is a possibility that assessing low-contrast detectability in vivo is different to phantom images as the task of clinical assessment by a radiologist, who has access to full image data and patient information, is relatively more complex compared to the simpler task of assessing subtle lesions in a phantom.

Paper III was a unique study design, as to the author's knowledge there are no studies that have compared the three phases of a CTU to evaluate image quality and pathology. Several different methods to reduce dose in CTU have been studied before [96, 97], but Paper III showed that a lowdose series seems to be sufficient as a first-line modality in certain patient groups and can be implemented into clinical practice to reduce patient dose. This might enlighten the medical community on the possible use of a low-dose abdominal CT as its implementation in clinical practice has been slow. However, radiologists should be aware of its limitations.

It should be noted that the results of the studies included in this thesis apply only to the specific modality, acquisition parameters and body part in each study and cannot be used to generalise abdominal dose optimisation in CT. Dose optimisation depends on the vendor, type of algorithm, CT scanner model and the complexity of the situation i.e. evaluation and comparison of several parameters and their effects on dose and image quality. One of the limitations of Paper IV indicates a need for further research to 
conduct cross-comparisons which could be valuable in evaluating image quality for thin slices of $\mathrm{AD} 5$ compared to the clinical standard of $3 \mathrm{~mm}$ slice thickness for $\mathrm{AD}_{3}$.

\section{Implications for patient care}

This thesis clinically validated the effect of IR in abdominal CT imaging and estimated potential dose reductions using two different classes of IR (statistical and model-based IR). The results of Paper I have been implemented in clinical practice. Results of Papers II and IV will be implemented in clinical practice to optimise abdominal CT protocols for the Somatom Force CT scanner. Results from paper III may increase clinical awareness for the value of the low-dose abdominal protocol when choosing an imaging method for certain patient groups who are more sensitive to radiation.

\section{Radiographers' role}

Radiographers as trained professionals have direct contact with the patient, and are responsible for obtaining the images and, hence delivering the absorbed dose. Awareness of parameters that affect dose and image quality as well as the principles of radiation protection are paramount for the profession. The switch from conventional radiographic $2 \mathrm{D}$ techniques to CT has affected the radiographers' practical task of image production. All scan protocol parameters are pre-set and are not apparent to the radiographers which makes it difficult to retain control over exposure parameters and radiation doses delivered [75]. Standardisation of protocols in CT is a basis for quality control and a practice for avoiding unnecessary variance in radiation doses delivered. The majority of patients can be examined using this standard protocol, but when the necessity arises to tailor the examination protocol to the patient's needs, solid knowledge of how change in parameters affect image quality and patient dose is necessary. General knowledge of CTDI $\mathrm{vol}_{\mathrm{v}}$ and DLP values in relation to DRLs and patient size may facilitate in judging if the dose to be delivered is appropriate. Hence, role expansion as described by Lundvall (2019) [75] with CPD is mandatory and especially important with the continuing technical development and increasing availability of new applications.

\section{Future aspects}

The development in CT during the past decade has been related to increasing detector width to meet the needs for high temporal resolution. But this trend seems to have reached a plateau and it is unlikely that it will continue. Today's focus is directed towards increasing the dose efficiency in new generation CTs. The benefits of diagnostic medical imaging shall continue to outweigh the low hypothetical risk, but judicious use of imaging is 
advocated. The foremost goal of medical imaging is delivering images of sufficient high image quality for diagnostic purposes i.e. dose optimisation and if dose reduction is achieved it is considered a bonus [22].

While the speed of introduction of novel clinical IR-algorithms is gradually slowing down, radiation dose reduction is still a challenging hot topic [39]. There is continuing innovative technical development within the field of artificial intelligence (AI), which has a potential to reduce CT radiation dose while shorter image reconstruction times are made possible [39]. The next generation of CT image reconstruction will be developed within the deep-learning framework [98]. Currently, neural networks (NN) have shown to improve image reconstruction with better noise suppression while maintaining anatomical structural fidelity. NNs are faster compared to their IR counterparts [98, 99].

Another new CT technology is photon-counting CT which counts individual photons while eliminating electronic noise [39]. Photon counting detectors can discriminate and divide the individual photon energies present in the transmitted x-ray beam [39].

New techniques present new challenges with regards to optimisation. These new challenges can be met by embracing new technology e.g. AI, and evolving work practices to meet the changing environment.

To get back to present practice, there are some unanswered questions remaining from Paper IV, where cross-comparisons of slice thickness variation with the different algorithm strengths could have provided valuable knowledge. Further research is therefore necessary to fill this gap.

Quality assurance in the X-ray department is a continuing practice and optimisation of scans of other body parts is necessary to keep the dose as low as reasonably achievable. Since many patients normally undergo thorax and abdominal examinations simultaneously, an extended ethical application was made to also include CT-thorax examinations for future dose reduction and optimisation studies. 
Visual grading evaluation and optimisation in abdominal Computed Tomography 


\section{CONCLUSIONS}

The main objective of this thesis was to assess the potential for radiation dose reduction with retained image quality using two classes of iterative reconstruction methods (statistical and model-based) in Papers I, II and IV, and to explore the diagnostic value of low-dose abdominal CT in Paper III.

The conclusions drawn from the individual papers are as follows:

\section{Paper I}

The SAFIRE iterative reconstruction algorithm improved image quality in low-dose abdominal CT. With a SAFIRE strength setting of 1 , the estimated dose reduction was rather small. The full potential of the algorithm remained unclear.

\section{Paper II}

The model-based iterative reconstruction algorithm ADMIRE showed improved image quality compared to Filtered back projection. A positive correlation between ADMIRE strength and increasing potential dose reduction was found for the majority, but not all, of the image criteria.

\section{Paper III}

Visualisation of renal anatomy in the three phases were as expected with each post-contrast phase showing favourable scores compared to the native phase. No statistically significant difference was found between the three phases for assessment of pathology. Since many certainty scores were in the high and medium categories, the LDCT seems to be sufficient to differentiate between normal and pathological examinations. In order to reduce the radiation burden in certain patient groups, the LDCT could be considered a suitable alternative as a first line imaging method. However, radiologists should be aware of its limitations.

\section{Paper IV}

As expected, when increasing slice thickness from $1 \mathrm{~mm}$ to $2 \mathrm{~mm}$ or $3 \mathrm{~mm}$, independent of the IR algorithm strength, a dose reduction was possible. ADMIRE5, in comparison to ADMIRE3, has diverse effects on the five 
image criteria depending on slice thickness and further dose reductions are limited to certain image criteria. Our findings do not support a general recommendation to replace ADMIRE3 by ADMIRE5 in clinical abdominal CT protocols. 


\section{ADDENDUM}

\section{Other published papers not included in the thesis}

Published paper related to but not part of the thesis

- Implications of patient centring on organ dose in Computed Tomography

Kataria B, Sandborg M and Althén Nilsson J

Radiation Protection Dosimetry, 2016:169; 130-135

Published paper not related to the thesis

- Patients preference for the examination of the large intestine with double contrast barium enema or Computed Tomography Colonography

Kataria B

Journal of Radiology Nursing 2011:30; 70-78

Other peer reviewed conference abstracts

- Implications of patient centring in Computed Tomography: a comparison between TLD measurements and CT Expo simulation

Kataria B, Sandborg M, Nilsson Althén J

Optimisation in X-ray and Molecular Imaging (OXMI), Gothenburg, Sweden 2015: Poster P-5

- Implications of patient centring in Computed Tomography: a comparison between TLD measurements and CT Expo simulation

Kataria B, Sandborg M, Nilsson Althén J

International Society of Radiographers and Radiological

Technicians (ISRRT) Seoul, Korea 2016: Oral presentation AFoo29 
Visual grading evaluation and optimisation in abdominal Computed Tomography

82 


\section{REFERENCES}

1. Smith-Bindman R, Lipson J, Marcus R, et al. Radiation dose associated with common computed tomography examinations and the associated lifetime attributable risk of cancer. Arch Intern Med. 2009;169(22):2078-86.

2. Shah NB, Platt SL. ALARA: is there a cause for alarm? Reducing radiation risks from computed tomography scanning in children. Current Opinion in Pediatrics. 2008;20(3):243-7.

3. ICRP: Managing patient dose in Multi-detector Computed Tomography (MDCT). Vol. 37. Exeter: Elsevier; 2007. (Valentin, editor. ICRP Publication 102).

4. Kalra MK, Maher MM, Toth TL, et al. Strategies for CT radiation dose optimisation. Radiology. 2004;230:619-28.

5. Wiest PW, Loeken JA, Heintz PH, et al. CT scanning A major source of radiation exposure. Semin Ultrasound CT. 2002;23(5):402-10.

6. Brenner DJ. Mimimising unwarranted Computed Tomography scans. Annals of ICRP. 2012;41:161-9.

7. Berrington de Gonzalez A, Mahesh M, Kim KP, et al. Projected cancer risks from computed tomographic scans performed in the United States in 2007. Arch Intern Med. 2009;169(22):2071-7. PubMed PMID: 20008689.

8. Brenner DJ, Hall EJ. Computed Tomography - An Increasing Source of Radiation Exposure. N Engl J Med 2007;357:2277-84.

9. Brenner DJ, Elliston CD, Hall EJ, et al. Estimated Risks of Radiation-Induced Fatal Cancer from Pediatric CT. American Journal of Roentgenology. 2001;176:289-96.

10. Chodick G, Ronckers CM, Shalev V, et al. Excess lifetime cancer mortality risk attributable to radiation exposure from CT. IMAJ. 2007;9:584-587.

11. De Jong PA, Mayo JR, Golmohammadi K, et al. Estimation of cancer mortality associated with repetitive computed tomography scanning. Am J Respir Crit Care Med. 2006;173(2):199-203.

12. Handee WR, $\mathrm{O}^{\prime}$ Connor MK. Radiation Risks of Medical imaging separating fact from fantasy. Radiology. 2012;264(2):312-21.

13. Siegel JA, Pennington CW. Mismeasure of Radiation Skeptic Debunking the Flawed Science that Low-dose Radiation May Cause Cancer; In Fact, It May Even be Beneficial. Skeptic Magazine. 2015;20(4):48-51.

14. Ulsh BA. Are Risks From Medical Imaging Still too Small to Be Observed or Nonexistent? Dose Response. 2015;13(1):1-27. 
15. Pearce MS, Salotti JA, Little MP, et al. Radiation exposure from CT scans in childhood and subsequent risk of leukaemia and brain tumours: a retrospective cohort study. The Lancet. 2012;380(9840):499-505.

16. Mathew JD, Forsythe AV, Brady Z, et al. Cancer risk in 680000 people exposed to computed tomography scans in childhood or adolescence:data linkage study of 11million Australians BMJ. 2013;346:f2360. doi: 10.1136/bmj.f236o.

17. Power SP, Moloney F, Twomey M, et al. Computed tomography and patient risk: Facts, perceptions and uncertainties. World J Radiol. 2016;8(12):902-915.

18. Rice HE, Frush DP, Farmer D, et al. Review of radiation risks from computed tomography: essentials for the pediatric surgeon. J Pediatr Surg. 2007;42(4):603-7.

19. Rawle M, Pighills A. Prevalence of unjustified emergency department $\mathrm{x}$-ray examination referrals performed in a regional Queensland hospital: A pilot study. J Med Radiat Sci. 2018;65(3):184-191.

20. Almén A, Leitz W, Richter S. National survey on justificatiion of CTexaminations in Sweden.2009 [cited 201913 September]:[1-30 p.]. https://www.stralsakerhetsmyndigheten.s.e/contentassets/7690aba 4a71d4fdo98afc223783f63a6/200903-national-survey-onjustification-of-ct-examinations-in-sweden

21. IAEA. Report of a consultation on justification of patient exposures in medical imaging. Radiat Prot Dosimetry. 2009;135(2):137-44.

22. Kalender W. Computed Tomography: Fundamentals, System Technology, Image quality, Applications. 3rd ed. Erlangen, Germany: Publicis Corporate Publishing; 2011.

23. Flohr T, B O, Schaller S. Multislice CT. In: Baert AL, Sartor K, editors. Multislice CT. Medical Radiology, Diagnostic Imaging. 2nd ed. Berlin, Heidelberg, Germany: Springer Verlag; 2004.

24. Mahesh M. MDCT Physics The Basics - Technology, Image Quality and Radiation dose. Philadelphia: Lippincott William \& Wilkins \& Wolters Kluwer; 2009.

25. Buzug T. Computed Tomography from Photon Statistics to Modern Cone-Beam CT. Berlin, Heidelberg: Springer Verlag; 2008.

26. Johnson TR. Dual-energy CT: general principles. AJR Am J Roentgenol. 2012;199(5 Suppl):S3-8.

27. Schmidt B, Brednhoeller C, Flohr T. Dual Source CT Imaging. In: Seidensticker PR, LK H, editors. Heidelberg: Springer Medizin Verlag; 2008.

28. CT Dose Reduction in the Abdomen \& Pelvis [Internet]. AAPM 2013. Available from: https://vimeo.com/channels/ctdose2013/77750525.

29. Strålsäkerhetsmyndighetens foreskrifter och allmanna rad om medicinska exponeringar.2018 [cited 201913 September]:[32 p.]. 
Report available at

https://www.stralsakerhetsmyndigheten.s.e/contentassets/5ca0970 e939642f68ac4bof5adfd391a/ssmfs-20185-

stralsakerhetsmyndighetens-foreskrifter-och-allmanna-rad-ommedicinska-exponeringar.pdf

30. ICRP: The 2007 recommendations of the International Commission on Radiological Protection. Vol. 37. Oxford, UK: Elsevier; 2007. (Valentin J, editor. ICRP publication 103).

31. IAEA. Radiation Protection and Safety in Medical Uses of Ionizing Radiation2018 [cited 201913

September]:[https://www.iaea.org/publications/11102/radiationprotection-and-safety-in-medical-uses-of-ionizing-radiation

32. Boice JD. The linear nonthreshold (LNT) model as used in radiation protection: an NCRP update. Int J Radiat Biol. 2017;93(10):10791092.

33. Shore RE, Beck HL, Boice JD Jr, et al. Recent Epidemiologic Studies and the Linear No-Threshold Model For Radiation ProtectionConsiderations Regarding NCRP Commentary 27. Health Phys. 2019;116(2):235-246.

34. Siegel JA, Welsh JS. Does Imaging Technology Cause Cancer? Debunking the Linear No-Threshold Model of Radiation Carcinogenesis. Technol Cancer Res Treat. 2016;15(2):249-56.

35. Kaza RK, Platt JF, Goodsitt MM, et al. Emerging techniques for dose optimisation in abdominal CT. RadioGraphics 2014;34:4-17.

36. Tube current modulation [Internet]. AAPM 2013 [cited 5 April, 2019]. Available from:

https://vimeo.com/channels/ctdose2013/77750520.

37. Goldman LW. Principles of CT and CT technology. J Nucl Med Technol. 2007;35(3):115-28

38. Pien H, Do S, Singh S, et al. Conventional and newer reconstruction techniques in CT. In: Tack D, Kalra M, Genevois PA, editors.

Radiation dose from multidetector CT. Berlin, Heidelberg: SpringerVerlag; 2012.

39. Willemink MJ, Noel PB. The evolution of image reconstruction for CT-from filtered back projection to artificial intelligence. Eur Radiol. 2019;29(5):2185-2195.

40. Stiller W. Basics of iterative reconstruction methods in computed tomography: A vendor-independent overview. Eur J Radiol. 2018;109:147-154.

41. Stierstorfer K, Rauscher A, Boese J, et al. Weighted FBP-a simple approximate 3D FBP algorithm for multislice spiral CT with good dose usage for arbitrary pitch. Phys Med Biol. 2004;49(11):22092218. doi: 10.1088/0031-9155/49/11/007. 
42. Beister M, Kolditz D, Kalender WA. Iterative reconstruction methods in X-ray CT. Phys Med. 2012;28(2):94-108.

43. Liu L. Model-based Iterative Reconstruction: A Promising Algorithm for Today's Computed Tomography Imaging. J Med Radiat Sci. 2014;45(2):131-136.

44. Kubo T. Vendor free basics of radiation dose reduction techniques for CT. Eur J Radiol. 2019;110:14-21. doi: 10.1016/j.ejrad.2018.11.002.

45. Grant K, Raupach R. Safire white paper2012 [cited 201820 December]:[https://pdfs.semanticscholar.org/138a/f76d2e121fbc79 gadf4e1a661b59c6494adf.pdf

46. Ramirez-Giraldo JC, Grant K, Raupach R. ADMIRE: Advanced Modeled Iterative Reconstruction2018 [cited 20199 January]:[https://static.healthcare.siemens.com/siemens hwemhwem ssxa websites-contextroot/wcm/idc/groups/public/@global/@imaging/@ct/documents/d ownload/mda4/ntq4/ edisp/ct whitepaper admire-05678345.pdf

47. Baumueller S, Winklehner A, Karlo C, et al. Low-dose CT of the lung: potential value of iterative reconstructions. Eur Radiol. 2012;22(12):2597-2606.

48. Schaller F, Sedlmair M, Raupach R, et al. Noise Reduction in Abdominal Computed Tomography Applying Iterative Reconstruction (ADMIRE). Acad Radiol. 2016;23(10):1230-8.

49. Månsson LG. Methods for the Evaluation of Image Quality A Review. Radiat Prot Dosim. 2000;90:89-99.

50. Whitson HM. Quantifying differences in CT image quality between a MBIR algorithm, an ASIR algorithm, and FBP. 2017. Located at: http://digitalcommons.ohsu.edu/etd/3913

51. Löve A. Optimisation of image quality and radiation dose in neuroradiological CT. Lund, Sweden: Lunds University; 2013.

52. Båth M. Evaluating imaging systems: practical applications. Radiat Prot Dosim. 2010;139(1-3):26-36.

53. Metz CE. Receiver operating characteristic analysis: a tool for the quantitative evaluation of observer performance and imaging systems. J Am Coll Radiol. 2006;3(6):413-22.

54. Båth M, Mansson LG. Visual grading characteristics (VGC) analysis: a non-parametric rank-invariant statistical method for image quality evaluation. Br J Radiol. 2007;80(951):169-76.

55. Smedby O, Fredrikson M. Visual grading regression: analysing data from visual grading experiments with regression models. Br $\mathrm{J}$

Radiol. 2010:1-9. doi: 10.1259/bjr/35254923.

56. Kumar R, Indrayan A. Receiver operating characteristics curve for medical researcher. Indian Pediatr. 2001;48:277-287. 
57. Park SH, Goo JM, Jo C-H. Receiver operating characteristics curve for radiologists. Korean J Radiol.5(1):11-18.

58. Tesselaar E, Dahlstrom N, Sandborg M. Clinical Audit of Image Quality in Radiology Using Visual Grading Characteristics Analysis. Radiat Prot Dosimetry. 2016;169(1-4):340-6.

59. Shrimpton P, Panzer W, Jessen K. European Guidelines on quality criteria for Computed Tomography.2000 [cited 201914 September]:[https://publications.europa.eu/s/jND6

60. Ludewig E, Richter A, Frame M. Diagnostic imaging--evaluating image quality using visual grading characteristic (VGC) analysis. Vet Res Commun. 2010;34(5):473-9.

61. Birkelo CC, Chamberlain WE, Phelps PS, et al. Tuberculosis case finding: A comparison of the effectiveness of various roentgenographic and photofluorographic methods. JAMA. 1947;133(6):359-366.

62. Drew T, Vo ML, Olwal A, et al. Scanners and drillers: characterizing expert visual search through volumetric images. J Vis. 2013;13(10).

63. Lundin M. Aspects on image quality in radiologic evaluation of the urinary tract. Linköping, Sweden: Linköping University; 2012 [cited 201912 September]. Available from: https://www.divaportal.org/smash/get/diva2:516406/FULLTEXTo1.pdf.

64. Hansson J, Mansson LG, Bath M. The Validity of Using Roc Software for Analysing Visual Grading Characteristics Data: An Investigation Based on the Novel Software Vgc Analyzer. Radiat Prot Dosim. 2016;169(1-4):54-9.

65. Smedby Ö, Fredrikson M, De Geer J, et al. Visual grading regression with random effects. SPIE Medical Imaging 2012; San Diego, California: SPIEDigitalLibrary.org/conference-proceedings-of-spie; 2012. p. 1-6.

66. Kvernby S. Optimization of activity level in rCBF SPECT using the observer study Visual Grading Regression. 2012. 42 p. Located at: http://lup.lub.lu.se/student-papers/record/3327138

67. Saffari SE, Love A, Fredrikson M, et al. Regression models for analyzing radiological visual grading studies--an empirical comparison. BMC Med Imaging. 2015;15:49.

68. Smedby Ö, Fredrikson M, De Geer J, et al. Quantifying the potential for dose reduction with visual grading regression. Brit $\mathbf{J}$ Radiol. 2013;86(1021):1-6. doi: 10.1259/bjr/31197714.

69. European Federation of Radiographer Societies . Electronic address iee. EFRS EQF level 6 Benchmark. www.efrs.eu; 2018.

70. Vanckaviciene A, Macijauskiene J, Blazeviciene A, et al. Assessment of radiographers' competences from the perspectives of radiographers and radiologists: a cross-sectional survey in Lithuania. BMC Med Educ. 2017;17(1):25. 
71. Mayo-Smith WW, Hara AK, Mahesh M, et al. How to do it managing radiation dose in CT. Radiology. 2014;273(3).

72. ESR, EFRS. Patient safety in medical imaging: A joint paper of the European Society of Radiology (ESR) and the European Federation of Radiographer Societies (EFRS). Radiography 2019;25(2):e26-e38. doi: 10.1016/j.radi.2019.01.009.

73. Justification Flow Chart ISRRT web page: ISRRT; [cited 201928 July]. Available from: https://www.isrrt.org/justification-flowchart

74. EFRS. European Qualification Framework Level 7 Benchmarking Document-Radiographers.2016 [cited 201925 August]:[https://api.efrs.eu/api/assets/publications/156

75. Lundvall LL. Radiography in Practice - Work and learning in medical imaging. Linköping, Sweden: Linköpings University; 2019. Available from: http://urn.kb.se/resolve?urn=urn:nbn:se:liu:diva-154931.

76. Verdun FR, Racine D, Ott JG, et al. Image quality in CT: From physical measurements to model observers. Phys Med. 2015;31(8):823-843.

77. Smith-Bindman, Miglioretti DL, Larson EB. Rising use of diagnostic medical imaging in a large integrated health system. Health Aff (Millwood). 2008;27(6):1491-502.

78. European population dose from medical imaging. Medical Radiation Exposure of the European Population Part 1/2 : Radiation protection no. 1802014 [cited 201825 August]:[Report available at https://ec.europa.eu/energy/sites/ener/files/documents/RP180.pdf

79. Standardiserade vårdförlopp. 2018 [cited 201817 December]:[https://www.cancercentrum.se/samverkan/varauppdrag/kunskapsstyrning/vardforlopp/

8o. Standardiserade vårdförlopp - Jämlik och effektiv vård med god kvalitet. 2019 [cited 201913 September]:

[https://skl.se/download/18.4fb1868016ac3ad3eo2a6o52/15584433 64284/Överenskommelse\%202019\%20om\%20standardiserade\%20 vårdförlopp\%20-

\%20Jämlik\%20och\%20effektiv\%2Ovård\%2omed\%2ogod\%2okvalitet\%20 2019.pdf

81. Tubiana M, Feinendegen LE, Yang C, et al. The Linear No-Threshold model is inconsistent with radiation biologic and experimental data. Radiology. 2009;251:13-22.

82. Berrington de González A, Darby S. Risk of cancer from diagnostic X-rays: estimates for the UK and 14 other countries. The Lancet. 2004;363(9406):345-351.

83. Cancer Statistics: Cancer Research UK [Internet]. 2017 [cited 5 April, 2019]. Available from: https://www.cancerresearchuk.org/healthprofessional/cancer-statistics/risk. 
84. Cancer statistik: Cancer Fonden [Internet]. [cited 5 April, 2019]. Available from: https://www.cancerfonden.s.e/om-cancer/statistik

85. Siegel RL, Miller KD, Jemal A. Cancer statistics, 2019. CA Cancer J Clin. 2019;69(1):7-34.

86. Wichmann JL, Hardie AD, Schoepf UJ, et al. Single- and dual-energy CT of the abdomen: comparison of radiation dose and image quality of 2nd and 3rd generation dual-source CT. Eur Radiol. 2016;27(2):642-650.

87. Ehman EC, Yu L, Manduca A, et al. Methods for clinical evaluation of noise reduction techniques in abdomino-pelvic CT. RadioGraphics 2014;34:849-862.

88. Halilic S, Kämmerling N. Värdet av DT-buköversikt som primär undersökning vid akut buk smärta2016 [cited 201913

September]:[1-22 p.]. Quality assessment report available at http://liu.divaportal.org/smash/get/diva2:1304503/FULLTEXTo1.pdf

89. Gordic S, Desbiolles L, Stolzmann P, et al. Advanced modelled iterative reconstruction for abdominal CT: Qualitative and quantitative evaluation. Clin Radiol. 2014;69(12):e497-e504. doi: 10.1016/j.crad.2014.08.012.

90. Kalra MK, Woisetschläger M, Dahlström N, et al. Radiation Dose Reduction With Sinogram Affirmed Iterative Reconstruction Technique for Abdominal Computed Tomography. J Comput Assist Tomogr 2012;36:339-346.

91. Greffier J, Fernandez A, Macri F, et al. Which dose for what image? Iterative reconstruction for CT scan. Diagn Interv Imaging. 2013;94(11):1117-21.

92. Ellmann S, Kammerer F, Allmendinger T, et al. Advanced Modeled Iterative Reconstruction (ADMIRE) Facilitates Radiation Dose Reduction in Abdominal CT. Acad Radiol. 2018;25(10):1277-1284. doi: 10.1016/j.acra.2018.01.014.

93. Larsson J, Bath M, Ledenius K, et al. Assessment of clinical image quality in paediatric abdominal CT examinations: dependency on the level of adaptiv statistical iterative rconstruction (ASiR) and the type of convolution kernel. Radiat Prot Dosimetry. 2016;169(1-4):123-9.

94. Euler A, Stieltjes B, Szucs-Farkas Z, et al. Impact of model-based iterative reconstruction on low-contrast lesion detection and image quality in abdominal CT: a 12-reader-based comparative phantom study with filtered back projection at different tube voltages. Eur Radiol. 2017;27(12):5252-5259.

95. Solomon J, Mileto A, Ramirez-Giraldo JC, et al. Diagnostic Performance of an Advanced MBIR for low contrast detectability with a third generation dual source multidetector scanner. Radiology. 2015; 275( 3):735-745. 
96. Dahlman P, Van der Molen AJ, Magnusson M, et al. How much dose can be saved in three-phase CT urography? A combination of normal-dose corticomedullary phase with low-dose unenhanced and excretory phases. AJR Am J Roentgenol. 2012;199(4):852-60.

97. Dyakov I AM, Groudeva V, Vassileva J, Stoinova V, Hadjidekov V. Optimisation of CT procedures in two radiology departments. Abstract Phys Medica. 2014;30.

98. Shan H, Padole A, Homayounieh F, et al. Competitive performance of a modularized deep neural network compared to commercial algorithms for low-dose CT image reconstruction. Nat Mach Intell. 2019;1(6):269-276.

99. Langlotz CP, Allen B, Erickson BJ, et al. A Roadmap for Foundational Research on Artificial Intelligence in Medical Imaging: From the 2018 NIH/RSNA/ACR/The Academy Workshop. Radiology. 2019;291(3):781-791. 
PAPER I 



\title{
Patient dose and image quality in low-dose abdominal CT: a comparison between iterative reconstruction and filtered back projection
}

\author{
Bharti Kataria1,2 and Örjan Smedby²,3 \\ ${ }^{1}$ Vrinnevi Hostpital, Norrköping; ${ }^{2}$ Department of Medicine and Health (IMH), Linköping University, Linköping; ${ }^{3}$ Center for Medical Image \\ Science and Visualization (CMIV), Linköping University, Linköping, Sweden \\ Correspondence to: Bharti Kataria. Email: Bharti.Kataria@lio.se
}

\begin{abstract}
Background: In computed tomography (CT), there is increasing concern for potential CT radiation hazards. Several raw-data-based iterative reconstruction techniques attempt to facilitate low-dose imaging without compromising image quality, which raises the question whether these techniques may allow further dose reduction.

Purpose: To compare image quality of iterative reconstruction and filtered back projection in low-dose abdominal CT and study the potential for further dose reduction.

Material and Methods: Forty-five patients underwent CT of the abdomen twice: with standard low-dose technique and with $30 \%$ reduced dose, using both iterative reconstruction and filtered back projection. Four radiologists made pair-wise image quality assessment using five visual criteria. Visual grading regression (VGR) and weighted kappa $\left(\kappa_{\mathrm{w}}\right)$ were used to analyze the data.

Results: There were significant effects of $\log (\mathrm{mAs})(P<0.001)$ and reconstruction algorithm $(P<0.01)$ on all image quality criteria with an estimated potential dose reduction of $5-9 \%$. Inter-observer agreement ranged from $70 \%$ to $91 \%$ and $\kappa_{\mathrm{w}}$ from -0.01 to 0.57 .

Conclusion: An iterative reconstruction algorithm improved image quality in abdominal CT, but the estimated dose reduction was rather small. The full potential of the algorithm remains unclear.
\end{abstract}

Keywords: Computed tomography, iterative reconstruction, image quality, filtered back projection (FBP)

Submitted June 25, 2012; accepted for publication December 16, 2012

Advancements in computed tomography (CT) technique have led to its wide use in diagnostic radiology as a standard modality with improvements in treatment and diagnosis of numerous medical conditions. There is, however, a cost attached to this advancement, as there is increasing concern for potential CT radiation hazards, which may elevate a person's lifetime risk of developing cancer. Although there may be some difficulty in quantifying lifetime risks for the individual person, this small risk, if applied to a large number of individuals, can result in a public health problem $(1,2)$.

It has been projected that approximately 29,000 future cancers could be related to the 7 million CT scans performed in the United States in 2007 (3). Similarly, the risk of developing cancer from CT coronary angiography examinations has been estimated as being 1 in 270 for women and 1 in
600 for men at the age of 40 years. At 20 years of age, this risk escalates to double and for 60-year-olds, it is half this amount (4). A recent retrospective cohort study on radiation exposure from CT scans in childhood, noted a positive association between radiation dose from CT scans to leukemia and brain tumors. The interpretation of the study results show that if use of CT scans in children delivers cumulative doses of about 50 milliGray (mGy) the risk for leukemia might be tripled and that doses of about $60 \mathrm{mGy}$ might triple the risk of brain cancer (5).

Dose reduction can be achieved by judicious use of CT as an imaging modality $(2,6)$. There are a multitude of dosesaving strategies available including examination-specific voltage settings, tube current modulation technique, and the use of specific scan protocols for the pediatric patients (2). In compliance with the "as low as reasonably 
Table 1 Scan parameters for standard low-dose abdominal CT and 30\% reduced dose protocols for a 128 slice Siemens Somatom Definition AS Scanner

\begin{tabular}{|c|c|c|c|c|c|c|c|c|c|}
\hline $\begin{array}{l}\text { Series } \\
\text { description }\end{array}$ & $\begin{array}{l}\text { Voltage } \\
\text { (kV) }\end{array}$ & $\begin{array}{l}\text { Quality reference } \\
\text { tube current time } \\
\text { product (mAs) }\end{array}$ & $\begin{array}{l}\text { Acquisition } \\
\text { mode }\end{array}$ & $\begin{array}{l}\text { Dose } \\
\text { modulation }\end{array}$ & $\begin{array}{l}\text { CARE } \\
\text { dose 4D }\end{array}$ & $\begin{array}{l}\text { Rotation } \\
\text { time }\end{array}$ & Pitch & $\begin{array}{l}\text { Collimation } \\
(\mathrm{mm})\end{array}$ & $\begin{array}{l}\text { Slice } \\
\text { thickness } \\
(\mathrm{mm})\end{array}$ \\
\hline $\begin{array}{l}\text { Standard low-dose } \\
\text { protocol }\end{array}$ & 120 & 50 & Axial & on & CARE Dose4D & 0.5 & 1.2 & 0.6 & 5 \\
\hline $\begin{array}{l}30 \% \text { reduced dose } \\
\text { protocol }\end{array}$ & 120 & 35 & Axial & on & CARE Dose4D & 0.5 & 1.2 & 0.6 & 5 \\
\hline
\end{tabular}

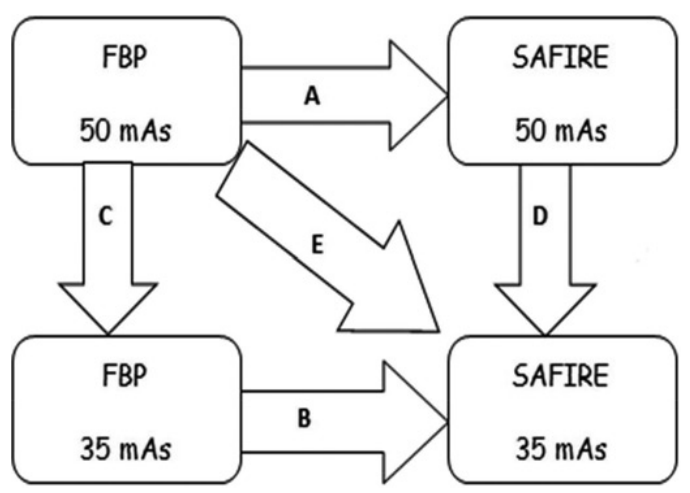

Fig. 1 Schematic diagram showing comparison of five pairs of image stacks for images acquired at $50 \mathrm{mAs}$ and $35 \mathrm{mAs}$ and reconstructed with SAFIRE and Filtered Back Projection (FBP)

achievable" (ALARA) principle, the optimization of CT protocols is necessary to meet the clinical need for accurate determination while maintaining sufficient image quality.

The standard image reconstruction algorithm is still filtered back projection (FBP), but during the last decade new CT scanners have enabled the reintroduction of iterative reconstruction methods which were used in the infancy of CT history $(7,8)$. Published studies indicate that this technique yields images with reduced image noise and artifacts (9-12).

Several raw-data-based iterative reconstruction techniques attempt to facilitate low-dose imaging without compromising image quality. It is thus of central clinical interest to study whether image quality with this reconstruction method is superior enough to allow further dose reduction.

This study focuses on efforts to reduce patient dose in abdominal CT by determining if the advantage of iterative reconstruction in terms of image quality is sufficient enough to facilitate dose reduction. We hypothesized that there is such a difference in image quality between iterative reconstruction and the FBP methods that there is room for further dose reduction with preserved image quality.

The aim of this study was to compare image quality with iterative reconstruction to filtered back projection in low-dose abdominal CT and to evaluate if further patient dose reduction is possible.

\section{Material and Methods}

This was a prospective study with a quantitative approach comprising a selection of patients presented to the radiology department at Vrinnevi Hospital, Norrköping for examination of a low-dose CT of the acute abdomen between October 2011 and February 2012.

\section{Sample selection}

A consecutive selection of 45 patients who underwent an acute abdominal CT presented to the radiology department. As radiation dose is cumulative for CT examinations, inclusion criterion was patients of at least 50 years of age and exclusion criterion was patients who had undergone more than four CT examinations over the past year. Non-Swedish speaking patients were excluded from this study as it was impossible to organize professional interpreter services for these examinations.

\section{Procedure}

Each patient was examined twice, using first a CT series of the abdomen with standard low-dose technique and then a second series with a $30 \%$ lower dose. Images were acquired in the clinical routine with a 128-slice Somatom Definition AS scanner (Siemens Medical Systems, Forcheim, Germany). The CT protocols employed the automatic exposure control technique for both the $x-y$ (transverse) and $\mathrm{z}$-(cranial-caudal) axis. Table 1 shows the parameters used for the two examinations.

For each examination, axial images were reconstructed from raw data both with the FBP algorithm and the iterative reconstruction algorithm SAFIRE strength 1 using B26f medium smooth ASA kernel, slice thickness $5 \mathrm{~mm}$ with a $2.5 \mathrm{~mm}$ reconstruction interval, and window settings 400/40 HU. The results were stored in the Picture Archiving and Communication System (PACS) (IDS7; Sectra Imtec AB, Linköping, Sweden). Patient demographic data were obtained from the radiological information system (RIS) (Sectra Imtec AB, Linköping, Sweden). A dose report (including scanner and dose parameters) was recorded for each patient. Body mass index (BMI), which was expected to influence image quality, was calculated for each patient.

Four radiologists, with CT experience in the range of 1-3 years (less experienced) and 20-22 years (experienced), independently performed visual image quality assessment comparing five pairs of image stacks from each patient (Fig. 1) resulting in 900 observations. 
Table 2 Patient scan and dose parameters presented as mean \pm standard deviation (SD) for images acquired at $50 \mathrm{mAs}$ and $35 \mathrm{mAs}$ and reconstructed with SAFIRE and filtered back projection (FBP)

\begin{tabular}{|c|c|c|c|}
\hline \multirow{2}{*}{$\begin{array}{l}\text { Body mass } \\
\text { index (BMI) }\end{array}$} & \multirow[b]{2}{*}{ Parameters } & \multicolumn{2}{|c|}{$\begin{array}{l}\text { Quality reference } \\
\text { (Q ref) tube current } \\
\text { time product (mAs) }\end{array}$} \\
\hline & & 50 & 35 \\
\hline $\mathrm{BMI}<30(n=30)$ & $\begin{array}{l}\text { DLP (mGy } \mathrm{cm}^{2} \text { ) } \\
\text { CTDI }_{\mathrm{vol}} \text { (mGy) } \\
\text { Effective mAs }\end{array}$ & $\begin{array}{c}125 \pm 38 \\
2.9 \pm 0.8 \\
42 \pm 12\end{array}$ & $\begin{array}{c}87 \pm 26 \\
2 \pm 0.6 \\
29 \pm 8\end{array}$ \\
\hline $\mathrm{BMI} \geq 30(n=15)$ & $\begin{array}{l}\text { DLP (mGy } \mathrm{cm}^{2} \text { ) } \\
\text { CTDI }_{\text {vol }} \text { (mGy) } \\
\text { Effective mAs }\end{array}$ & $\begin{array}{c}197 \pm 43 \\
4.7 \pm 0.8 \\
64 \pm 12\end{array}$ & $\begin{aligned} 137 & \pm 31 \\
3 & \pm 0.6 \\
44 & \pm 9\end{aligned}$ \\
\hline
\end{tabular}

$\mathrm{CTDI}_{\text {vol }}$, computed tomography dose index volume; DLP, dose length product

The image stacks were shown in pair-wise random order with respect to dose level and image reconstruction, and all demographic and scanner data were removed at the PACS workstation so that the radiologists were blinded. Readings were performed according to European Guidelines on quality criteria for abdominal CT (13) on regular DICOM-calibrated PACS workstations. The image quality was evaluated at similar anatomic sites in the abdomen for each patient.

The criteria used were as follows: 1 , visually sharp reproduction of the intestine; 2 , visually sharp reproduction of the pancreatic contours; 3 , visually sharp reproduction of the kidneys and proximal ureters; 4, visually sharp reproduction of the aorta; and 5, critical reproduction of the gallbladder wall.

Image quality was graded on a 5-point Likert-type scale with the following scores: -2 , image on left monitor is definitely better than image on right monitor; -1 , image on left monitor is probably better than image on right monitor; 0 , images on left and right monitors are equivalent; +1 , image on right monitor is probably better than image on left monitor; and +2 : image on right monitor is definitely better than image on left monitor.

A sixth criterion was added to assess the visualization of pathology with the exclusion of diverticulosis and similar age-related findings. This criterion was also assessed on a 5-point Likert-type scale as follows: 1, normal examination; 2 , probably normal examination; 3 , inconclusive examination; 4, probably pathological examination; and 5, pathological examination.

\section{Statistical analysis}

The scores were entered into a spreadsheet and statistically analyzed using visual grading regression (VGR), in which ordinal logistic regression is applied to scores from observer ratings while controlling for dependencies between observers, patients, and methods (14) using STATA 10.1 (Stata Corporation LP, College Station, TX, USA). As standard statistical software does not allow for random effects in logistic regression, the STATA software module Generalized Linear and Latent Mixed Models (GLLAMM) was applied to take into consideration random effects due to individual patients and radiologists, who can both be seen as random samples from larger populations (15-17). The potential for dose reduction was quantified by relating the VGR coefficients for $\log (\mathrm{mAs})$ and SAFIRE reconstruction to each other, as described in (16). The principle underlying this calculation is to relate the differences in image quality score brought about by changing the reconstruction method to that brought about by changing the dose. Formally, if the regression coefficient of $\log (\mathrm{mAs})$ is $a$ and that of iterative reconstruction is $b$, then the dose reduction is given by $1-\exp (-b / a)$. Weighted kappa $\left(\kappa_{\mathrm{w}}\right)$ was used to describe inter-observer agreement between the four readers. The significance limit was set at $P=0.05$.

\section{Ethical aspects}

Approval was obtained from the regional Ethics and Radiation Protection committees to carry out two CT examinations on each patient included in this study. Informed written consent was obtained from each patient before the examination with information that participation in the study was voluntary and their right to withdraw from the study at any time. The images were displayed for the reading radiologists with all patient identification data removed thereby making it impossible to identify any individual patient.

\section{Results}

There were 45 patients ( 24 men, 21 women; age range, 50-95 years; mean age, 65 years; standard deviation $[\mathrm{SD}] \pm 9.6$; BMI range, 17.1-46.8; mean BMI, 28.21; $\mathrm{SD} \pm 5.6)$. Patient scan data and dose parameters are presented in Table 2.

There were a total of 12 missing values, scores for criterion 5 (assessment of gall bladder wall), mostly due to the observer not being able to identify any gallbladder. These have not been included in the statistical analysis.

The distribution of the scores for the five evaluation criteria assessed is shown in (Figs. 2-6). The scores for SAFIRE versus FBP, when comparing pairs at the same dose level (left two stacked bars in each figure), were mostly around 0 , indicating that image quality was often judged as equivalent in the two images for all the assessed criteria. However, the scores in favor of SAFIRE $(+1$ and +2 ) were consistently slightly more common than those in favor of FBP ( -1 and -2). Comparisons between two dose levels with the same algorithm gave, not surprisingly, higher values to the higher dose level (many cases with -1 and -2 in the third and fourth stacked bar). There was deterioration of image quality when comparing images for SAFIRE at $35 \mathrm{mAs}$ with FBP at $50 \mathrm{mAs}$, with the scores more in favor of FBP 50 mAs (rightmost stacked bars).

Scores for the assessment of pathology in pair-wise comparison for all observations are shown in Fig. 7. Neither mAs nor type of reconstruction algorithm showed any effect on the distribution of the scores.

When analyzing all the data in one statistical model, the VGR coefficients (Table 3 ) revealed a strongly significant effect of $\log (\mathrm{mAs})(P<0.001)$ and a significant effect of 


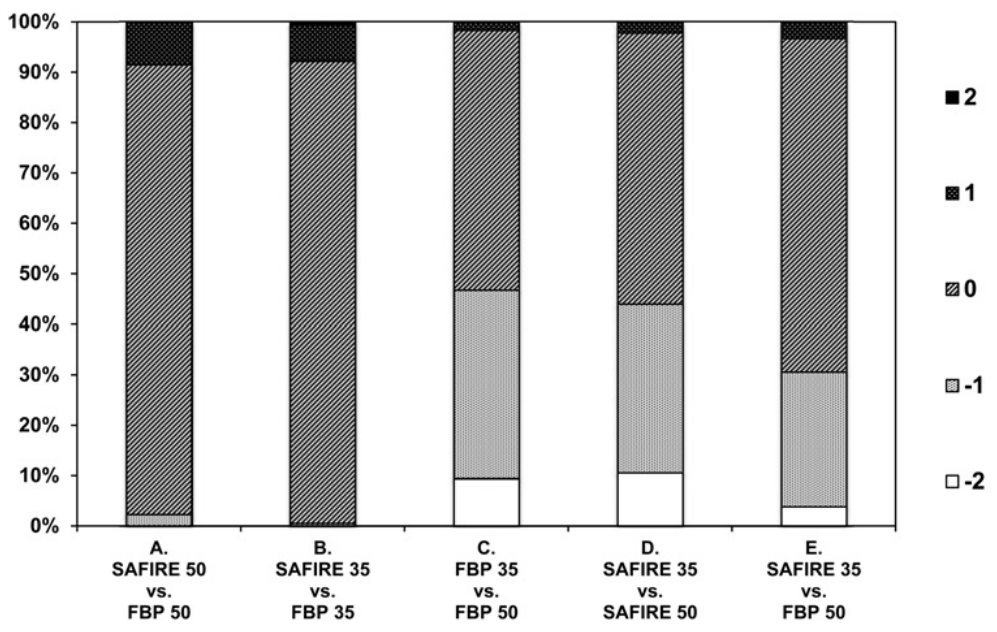

Fig. 2 Criterion 1: Visually sharp reproduction of the intestine. Distribution of scores for pair-wise comparison of images acquired at $50 \mathrm{mAs}$ and $35 \mathrm{mAs}$ and reconstructed with SAFIRE and filtered back projection (FBP). A positive score $(+1$ or +2$)$ in the leftmost bar indicates that SAFIRE 50 mAs was rated better than FBP $50 \mathrm{mAs}$, whereas a negative score $(-1$ or -2$)$ indicates that FBP $50 \mathrm{mAs}$ was rated better than SAFIRE $50 \mathrm{mAs}$, and so forth

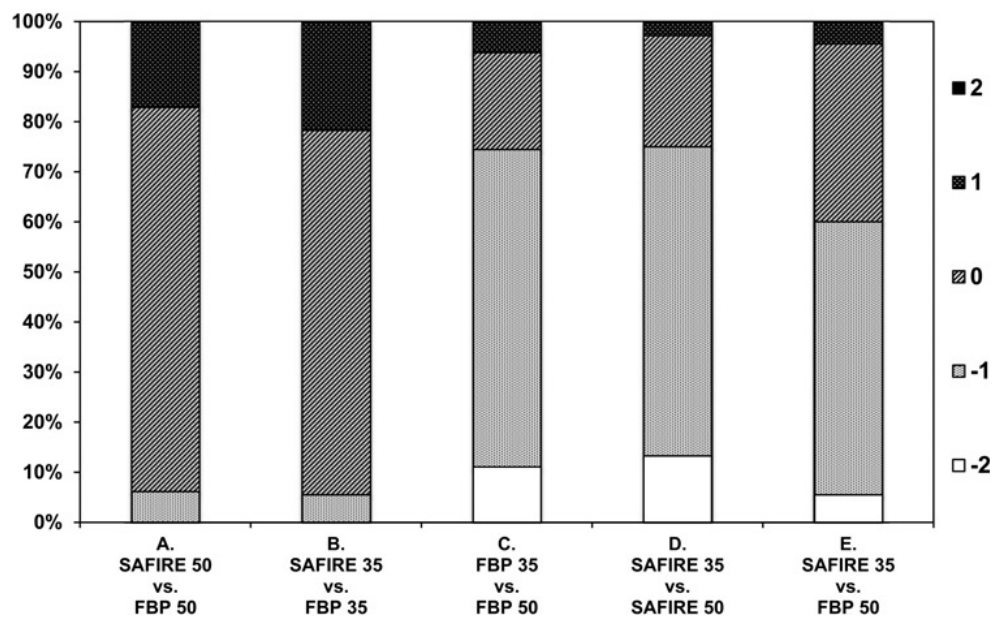

Fig. 3 Criterion 2: Visually sharp reproduction of the pancreatic contours. Distribution of scores for pair wise comparison of images acquired at 50 mAs and 35 $\mathrm{mAs}$ and reconstructed with SAFIRE and filtered back projection (FBP). A positive score $(+1$ or +2$)$ in the leftmost bar indicates that SAFIRE 50 mAs was rated better than FBP $50 \mathrm{mAs}$, whereas a negative score $(-1$ or -2$)$ indicates that FBP $50 \mathrm{mAs}$ was rated better than SAFIRE $50 \mathrm{mAs}$, and so forth

SAFIRE reconstruction $(P<0.01)$, on all image criteria. This is illustrated by higher coefficient values for $\log (\mathrm{mAs})$ and somewhat lower for SAFIRE reconstruction. The estimated dose reduction was rather small, ranging from $5 \%$ for sharp reproduction of the gallbladder wall to $9 \%$ for reproduction of the aorta, kidneys, and proximal ureters.

Inter-observer reliability was calculated by using weighted pair-wise kappa $\left(\kappa_{\mathrm{w}}\right)$ for all four readers to see if there was any difference between experienced readers and readers with less experience. Table 4 illustrates the percentage of agreement (range, 70-93\%) and kappa values (range, $-0.01-0.57)$ for comparison between pairs of experienced and inexperienced readers. Kappa values showed a significant $(P<0.001)$, fair to moderate agreement for experienced readers for all the criteria assessed. With the inexperienced readers, the results were more variable. When agreement and kappa were calculated separately for images reconstructed with SAFIRE and FBP, no clear differences emerged (data not shown).

To compare variations in estimation of dose reductions due to BMI the population was divided into two groups: BMI $<30 \mathrm{~kg} / \mathrm{m}^{2}(n=30)$ and BMI $\geq 30 \mathrm{~kg} / \mathrm{m}^{2} \quad(n=15)$. 


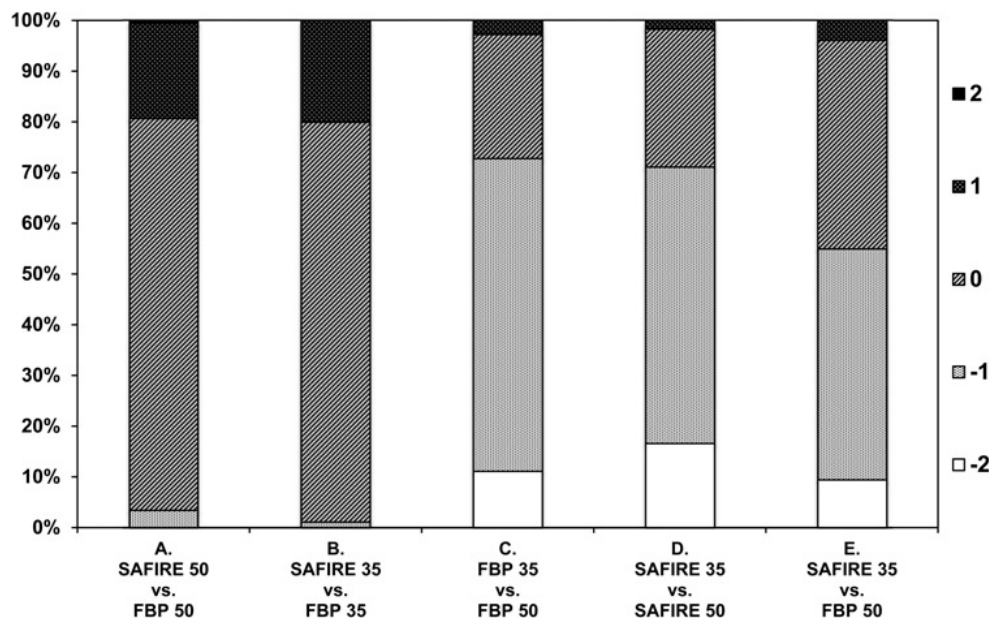

Fig. 4 Criterion 3: Visually sharp reproduction of the kidneys and proximal ureters. Distribution of scores for pair-wise comparison of images acquired at $50 \mathrm{mAs}$ and $35 \mathrm{mAs}$ and reconstructed with SAFIRE and filtered back projection (FBP). A positive score ( +1 or +2$)$ in the leftmost bar indicates that SAFIRE $50 \mathrm{mAs}$ was rated better than FBP $50 \mathrm{mAs}$, whereas a negative score $(-1$ or -2$)$ indicates that FBP $50 \mathrm{mAs}$ was rated better than SAFIRE $50 \mathrm{mAs}$, and so forth

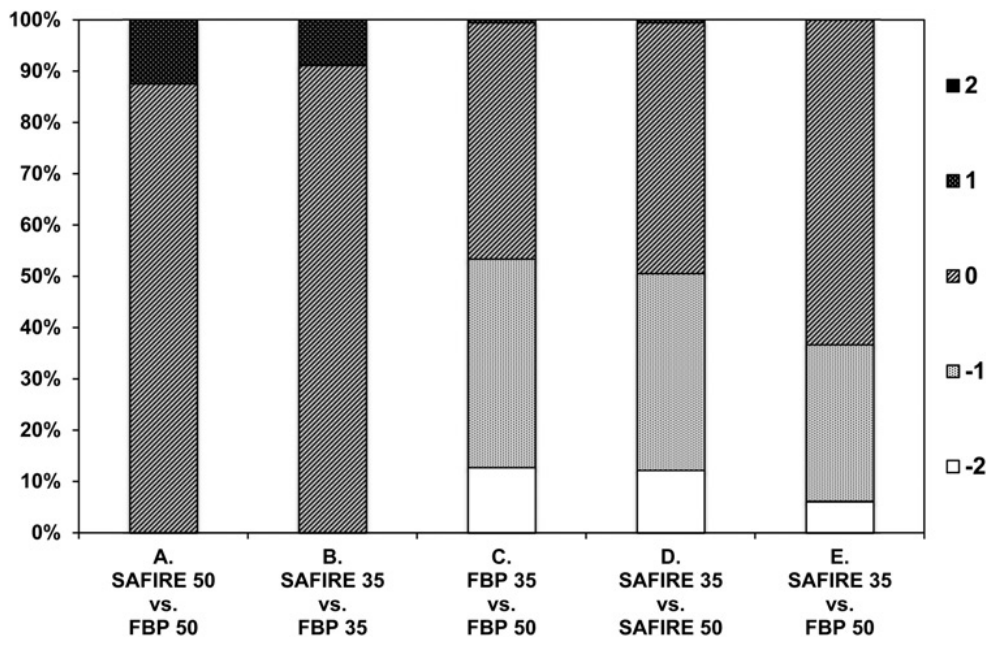

Fig. 5 Criterion 4: Visually sharp reproduction of the aorta. Distribution of scores for pair-wise comparison of images acquired at $50 \mathrm{mAs}$ and $35 \mathrm{mAs}$ and reconstructed with SAFIRE and filtered back projection (FBP). A positive score $(+1$ or +2$)$ in the leftmost bar indicates that SAFIRE 50 mAs was rated better than FBP $50 \mathrm{mAs}$, whereas a negative score $(-1$ or -2$)$ indicates that FBP $50 \mathrm{mAs}$ was rated better than SAFIRE $50 \mathrm{mAs}$, and so forth

The VGR analysis shows that dose reduction estimates were slightly higher for the group with BMI $<30 \mathrm{~kg} / \mathrm{m}^{2}$ (Table 5) compared to the group with BMI $\geq 30 \mathrm{~kg} / \mathrm{m}^{2}$ (Table 6).

\section{Discussion}

CT technology continues to evolve, leading to an increase in its use as an imaging modality. Although the CT scans are medically beneficial, they have a higher radiation dose level compared to conventional X-rays. There is growing concern for potential cancer risks associated with irradiation making this a public health issue based on estimations from several studies which raise concerns regarding the continual increase in use of CT as an imaging modality (3-5). Emphasis should be placed on the benefit-risk ratio where benefit of CT scan outweighs the risk if the examination is medically justified $(18,19)$. The reintroduction of iterative reconstruction technique is an important factor to be considered when optimizing clinical CT protocols. The processing time for iterative reconstruction has limited its use in clinical radiology, but with modern computer 


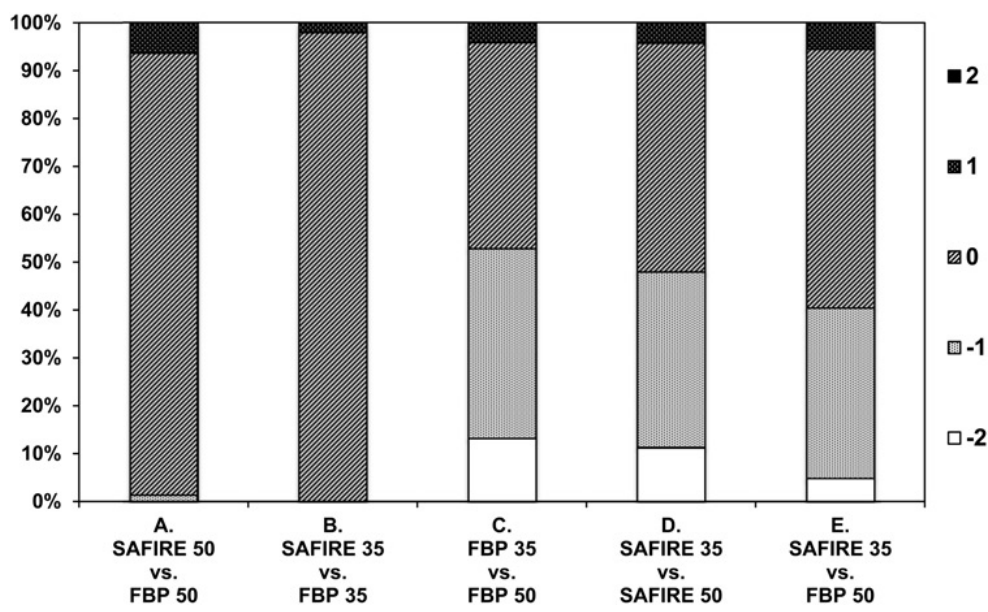

Fig. 6 Criterion 5: Critical reproduction of the gallbladder wall. Distribution of scores for pair-wise comparison of images acquired at $50 \mathrm{mAs}$ and $35 \mathrm{mAs}$ and reconstructed with SAFIRE and filtered back projection (FBP). A positive score $(+1$ or +2$)$ in the leftmost bar indicates that SAFIRE 50 mAs was rated better than FBP $50 \mathrm{mAs}$, whereas a negative score $(-1$ or -2$)$ indicates that FBP $50 \mathrm{mAs}$ was rated better than SAFIRE $50 \mathrm{mAs}$, and so forth

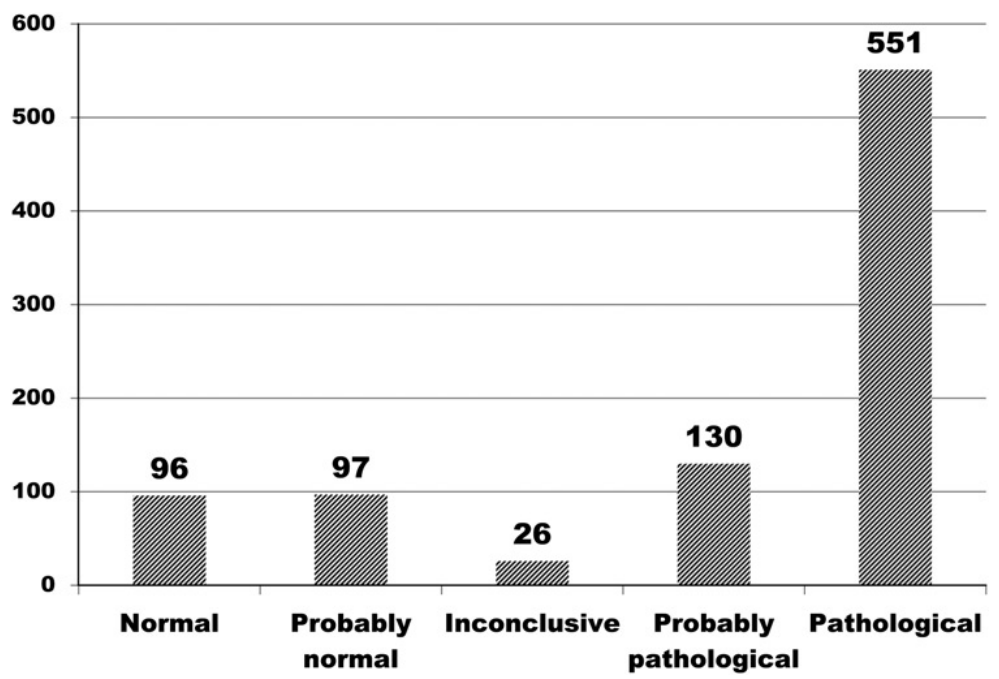

Fig. 7 Criterion 6: Presence of pathology excluding diverticulosis and other age-related findings. Distribution of scores for pair-wise comparison of images acquired at $50 \mathrm{mAs}$ and $35 \mathrm{mAs}$ and reconstructed with SAFIRE and filtered back projection (FBP) distribution of the scores

hardware and software, SAFIRE reconstruction times are today similar to those for FBP, thus facilitating wide use of this technique in both emergency and non-emergency CT examinations.

This study presents a clinical evaluation of subjective image quality of Sinogram Affirmed Iterative Reconstruction (SAFIRE) with repeat examinations of the same individual using low-dose and reduced-dose technique. This gives a realistic comparison of image quality between the two techniques in order to evaluate radiation dose reduction. Ethical issues involved were resolved by obtaining approval from the regional Ethics and Radiation Protection committees and by restricting population age selection to 50 years and over.

The inter-observer agreement ranged from poor to moderate with significant kappa values only when comparing the experienced readers. As the assessment is subjective, it is likely that variation does occur even when the same reader assesses the same pair of images on two different occasions. This is common practice in radiology departments today, for quality assurance purposes of the examination reports, where a second interpretation either confirms or reviews the report from the first reading. In experimental studies like the present study, it is of importance to have 
Table 3 VGR coefficient for all the criteria with estimated dose reduction values for pair-wise comparison of images acquired at $50 \mathrm{mAs}$ and $35 \mathrm{mAs}$ and reconstructed with SAFIRE and filtered back projection (FBP)

\begin{tabular}{|c|c|c|c|}
\hline \multirow[b]{2}{*}{ Criterion } & \multicolumn{2}{|c|}{$\begin{array}{l}\text { Regressions } \\
\text { coefficients }\end{array}$} & \multirow{2}{*}{$\begin{array}{l}\text { Estimated } \\
\text { dose } \\
\text { reduction } \\
(\%, 95 \% \mathrm{Cl}\end{array}$} \\
\hline & $\log (m A s)$ & $\begin{array}{l}\text { SAFIRE } \\
\text { reconstruction }\end{array}$ & \\
\hline $\begin{array}{l}\text { 1. Visually sharp } \\
\text { reproduction of the } \\
\text { intestine }\end{array}$ & $6.95^{*}$ & $0.61^{*}$ & $8(6-11)$ \\
\hline $\begin{array}{l}\text { 2. Visually sharp } \\
\text { reproduction of the } \\
\text { pancreatic contours }\end{array}$ & $7.80^{*}$ & $0.55^{*}$ & $7(5-9)$ \\
\hline $\begin{array}{l}\text { 3. Visually sharp } \\
\text { reproduction of the } \\
\text { kidneys and proximal } \\
\text { ureters }\end{array}$ & $8.56^{*}$ & $0.79^{*}$ & $9(7-11)$ \\
\hline $\begin{array}{l}\text { 4. Visually sharp } \\
\text { reproduction of } \\
\text { the aorta }\end{array}$ & $9.49^{*}$ & $0.94^{*}$ & $9(7-12)$ \\
\hline $\begin{array}{l}\text { 5. Critically sharp } \\
\text { reproduction of the } \\
\text { gallbladder wall }\end{array}$ & $6.87^{*}$ & $0.36^{\dagger}$ & $5(2-8)$ \\
\hline
\end{tabular}

${ }^{*} P<0.001$

${ }^{\dagger} P<0.01$

several readers in order to achieve a good inter-observer reliability.

The blinding of the radiologists to all scanning and patient data reduces the risk for systematic errors occurring during assessment of image quality. Assessment of image quality was carried out on DICOM-calibrated PACS workstations with which the radiologists were familiar, thus reducing risk for random errors.

This study used test protocols with similar $\mathrm{kV}$. The option to use a lower voltage such as $100 \mathrm{kV}$ might have shown better results; however, the purpose in this first study was to isolate one factor (mAs) affecting image quality in order to avoid difficulties in interpretation of the results.

Subjective image quality is one of the corner-stones in the assessment of the diagnostic quality of an image. According to the ALARA principle, the amount of noise acceptable in an image is assessed to determine radiation dose necessary without compromising the diagnostic performance. The question that arises is "how low can one go?", as methods for reducing CT dose have an impact on image quality as well. Balance between dose and image quality can be achieved by understanding the relation between newer CT applications and technology and CT dose (20). Of course, new reconstruction methods with improved balance between image quality and dose may permit either reduced dose at preserved image quality, or improved image quality at preserved dose. In this study, however, we have focused on the potential for dose reduction.

If iterative reconstruction allows for dose reduction by reducing image noise, this will facilitate optimization of dose protocols. Clinical trials similar to this study could be carried out in order to optimize protocols, but the ethical issues involved with extra irradiation of the patient might limit the study population. However, VGR offers a method to quantify dose reduction and can be particularly useful in pilot studies where the actual testing and evaluation of dose reduction for certain measures can be estimated before designing the main study for testing its diagnostic value (16).

In the present study, an assumed dose reduction of $30 \%$ on a low-dose series was based on manufacturer recommendations suggesting up to $60 \%$ dose reduction in full-dose examinations. With the results at hand, these figures were overly optimistic for low-dose abdominal examinations. To our knowledge, there are no comparative low-dose abdominal studies like the present study on the SAFIRE algorithm. A recent study of Kalra et al. (21) assessed the effect of SAFIRE and FBP on abdominal CT performed with standard dose 100\% (Qref, $200 \mathrm{mAs}$ ) compared to 50\% (Qref, $100 \mathrm{mAs}$ ) and 75\% (Qref, $50 \mathrm{mAs}$ ) radiation dose reductions. They concluded that SAFIRE provides images with no loss in diagnostic value at 50\% reduced dose and in some patients also at $75 \%$ reduced dose. There are two other studies, both utilizing CT angiography protocols, that show that SAFIRE image noise was significantly lower in half-dose data-sets compared to full-dose data-sets from FBP, indicating a potential for dose reductions by $>50 \%(22,23)$. One should bear in mind that the potential for dose reduction may be smaller in low-dose abdominal examinations, where strict optimization has already produced a protocol near the border of clinical acceptability. It is possible that greater dose reduction could have been achieved if the same methodology had been applied to standard CT protocols.

SAFIRE is available in five strengths from 1 to 5 , where an increase in SAFIRE strength setting leads to a reduction in image noise. Still, the unfamiliar "plastic" appearance of these images may seem strange for unaccustomed readers, which in the beginning could lead to poorer diagnostic performance (24). In this study, clinical setting SAFIRE strength 1 was used, as it yields a somewhat comparable appearance to the standard FBP. The effect of SAFIRE strength 1 was rather small, and did not compensate for the increase in noise due to dose reduction when comparing series at 50 $\mathrm{mAs}$ and $35 \mathrm{mAs}$, as shown by the estimates of $5-9 \%$ in dose reduction.

With SAFIRE software upgrade of the scanner in September 2011, the clinical routine dose was reduced by $30 \%$ for all CT protocols, and to date this dose reduction is still used for all other protocols except for the low-dose abdominal CT where the reduction from $50 \mathrm{mAs}$ to $35 \mathrm{mAs}$ yielded images of unacceptable quality. As there were concerns about the diagnostic accuracy of the examination, the routine dose was increased from $35 \mathrm{mAs}$ to $45 \mathrm{mAs}$ in October 2011, immediately before the start of the present study. This is close to the dose reductions estimated from our study.

Possibly, a higher SAFIRE strength could have given higher dose reduction estimation values. A gradual increase in SAFIRE strength could enable radiologists to leave their comfort zone and adapt to the unfamiliar appearance of iteratively reconstructed images without having to compromise diagnostic accuracy. According to previous experience in iterative reconstruction imaging techniques, this adaptation should take place within a short period of time (25). 
Table 4 Weighted pair-wise Kappa $\left(\kappa_{\mathrm{w}}\right)$ and agreement (\%) scores for evaluation of criteria in pair-wise comparison between experienced and less experienced readers of images acquired at $50 \mathrm{mAs}$ and $35 \mathrm{mAs}$ and reconstructed with SAFIRE and filtered back projection (FBP)

\begin{tabular}{|c|c|c|c|c|c|c|}
\hline \multirow{2}{*}{ Criterion } & \multicolumn{2}{|c|}{$\begin{array}{l}\text { Experienced } v s . \\
\text { experienced }\end{array}$} & \multicolumn{2}{|c|}{$\begin{array}{l}\text { Less experienced } v s . \\
\text { less experienced }\end{array}$} & \multicolumn{2}{|c|}{$\begin{array}{l}\text { Experienced vs. less } \\
\text { experienced }\end{array}$} \\
\hline & $\begin{array}{l}\text { Agreement } \\
(\%)\end{array}$ & $\begin{array}{l}\kappa_{\mathrm{w}} \\
(95 \% \mathrm{Cl})\end{array}$ & $\begin{array}{l}\text { Agreement } \\
(\%)\end{array}$ & $\begin{array}{l}\kappa_{\mathrm{w}} \\
(95 \% \mathrm{Cl})\end{array}$ & $\begin{array}{l}\text { Agreement } \\
(\%)\end{array}$ & $\begin{array}{l}\kappa_{\mathrm{w}} \\
(95 \% \mathrm{Cl})\end{array}$ \\
\hline 1. Visually sharp reproduction of the intestine & 87 & $\begin{array}{l}0.21^{*} \\
(0.15-0.27)\end{array}$ & 84 & $\begin{array}{l}0.03^{\dagger} \\
(0.01-0.05)\end{array}$ & $84-91$ & $\begin{array}{l}-0.01-0.39^{\ddagger} \\
(-0.45-0.47)\end{array}$ \\
\hline $\begin{array}{l}\text { 2. Visually sharp reproduction of the pancreatic } \\
\text { contours }\end{array}$ & 91 & $\begin{array}{l}0.57^{*} \\
(0.49-0.65)\end{array}$ & 84 & $\begin{array}{l}0.35^{*} \\
(0.26-0.45)\end{array}$ & $84-87$ & $\begin{array}{r}0.35-0.44^{*} \\
(0.27-0.53)\end{array}$ \\
\hline $\begin{array}{l}\text { 3. Visually sharp reproduction of the kidneys and } \\
\text { proximal ureters }\end{array}$ & 90 & $\begin{array}{l}0.45^{*} \\
(0.38-0.53)\end{array}$ & 85 & $\begin{array}{l}0.42^{*} \\
(0.33-0.50)\end{array}$ & $84-86$ & $\begin{array}{r}0.33-0.45^{*} \\
(0.25-0.53)\end{array}$ \\
\hline 4. Visually sharp reproduction of the aorta & 89 & $\begin{array}{l}0.36^{*} \\
(0.29-0.43)\end{array}$ & 81 & $\begin{array}{l}0.05^{*} \\
(0.02-0.08)\end{array}$ & $84-93$ & $\begin{array}{r}0.12-0.44^{*} \\
(0.05-0.52)\end{array}$ \\
\hline $\begin{array}{l}\text { 5. Critically sharp reproduction of the gallbladder } \\
\text { wall }\end{array}$ & 91 & $\begin{array}{l}0.46^{*} \\
(0.37-0.54)\end{array}$ & 84 & $\begin{array}{l}0.04^{\ddagger} \\
(-0.02-0.11)\end{array}$ & $85-91$ & $\begin{array}{r}0.13-0.37^{\ddagger} \\
(0.07-0.47)\end{array}$ \\
\hline 6. Is there any pathology present? & 84 & $\begin{array}{l}0.53^{*} \\
(0.41-0.65)\end{array}$ & 72 & $\begin{array}{l}0.23^{*} \\
(0.16-0.30)\end{array}$ & $70-86$ & $\begin{array}{r}0.28-0.47^{*} \\
(0.22-0.57)\end{array}$ \\
\hline
\end{tabular}

${ }^{*} P<0.001$

${ }^{\dagger} P<0.01$

${ }^{\ddagger}$ Not significant

$\mathrm{Cl}$, confidence interval

Table 5 VGR coefficient body mass index (BMI) $<30$ for all the criteria with estimated dose reduction values for pair-wise comparison of images acquired at $50 \mathrm{mAs}$ and $35 \mathrm{mAs}$ and reconstructed with SAFIRE and filtered back projection (FBP)

\begin{tabular}{|c|c|c|c|}
\hline \multirow[b]{2}{*}{ Criterion } & \multicolumn{2}{|c|}{ Regression coefficients } & \multirow{2}{*}{$\begin{array}{l}\text { Estimated } \\
\text { dose reduction } \\
(\%, 95 \% \mathrm{Cl})\end{array}$} \\
\hline & $\log (m A s)$ & $\begin{array}{l}\text { SAFIRE } \\
\text { reconstruction }\end{array}$ & \\
\hline $\begin{array}{l}\text { 1. Visually sharp } \\
\text { reproduction of } \\
\text { the intestine }\end{array}$ & $6.28^{*}$ & $0.58^{*}$ & $9(5-13)$ \\
\hline $\begin{array}{l}\text { 2. Visually sharp } \\
\text { reproduction of } \\
\text { the pancreatic } \\
\text { contours }\end{array}$ & $7.37^{*}$ & $0.55^{*}$ & $7(4-10)$ \\
\hline $\begin{array}{l}\text { 3. Visually sharp } \\
\text { reproduction of } \\
\text { the kidneys and } \\
\text { proximal ureters }\end{array}$ & $7.80^{*}$ & $0.75^{*}$ & $9(7-12)$ \\
\hline $\begin{array}{l}\text { 4. Visually sharp } \\
\text { reproduction of } \\
\text { the aorta }\end{array}$ & $9.05^{*}$ & $1.00^{*}$ & $10(8-13)$ \\
\hline $\begin{array}{l}\text { 5. Critically sharp } \\
\text { reproduction of } \\
\text { the gallbladder } \\
\text { wall }\end{array}$ & $5.80^{*}$ & $0.31^{\dagger}$ & $5(1-10)$ \\
\hline
\end{tabular}

$$
{ }^{*} P<0.001
$$$$
{ }^{\dagger} P<0.05
$$

This study did not assess artifacts or image noise but ring artifacts were observed on CT low-dose images from the scanner during the course of this study. It is possible that this could have affected the overall subjective assessment of the criteria on the $35 \mathrm{mAs}$ images as artifacts affect image quality.

For smaller patients, if automatic exposure control (AEC) is not employed, the noisy images due to photon starvation may affect the criteria for the anatomical sites in the abdomen by impairing visibility due to lack of fat around the abdominal organs (9). However, the use of dose reduction applications (Care Dose $4 \mathrm{D}$ ) and the scanner
Table 6 VGR coefficient body mass index (BMI) $>30$ for all the criteria with estimated dose reduction values for pair-wise comparison of images acquired at $50 \mathrm{mAs}$ and $35 \mathrm{mAs}$ and reconstructed with SAFIRE and filtered back projection (FBP)

\begin{tabular}{|c|c|c|c|}
\hline \multirow[b]{2}{*}{ Criterion } & \multicolumn{2}{|c|}{ Regression coefficients } & \multirow{2}{*}{$\begin{array}{l}\text { Estimated } \\
\text { dose reduction } \\
(\%, 95 \% \mathrm{Cl})\end{array}$} \\
\hline & $\log (m A s)$ & $\begin{array}{l}\text { SAFIRE } \\
\text { reconstruction }\end{array}$ & \\
\hline $\begin{array}{l}\text { 1. Visually sharp } \\
\text { reproduction of } \\
\text { the intestine }\end{array}$ & $8.48^{*}$ & $0.66^{\dagger}$ & $8(3-12)$ \\
\hline $\begin{array}{l}\text { 2. Visually sharp } \\
\text { reproduction of } \\
\text { the pancreatic } \\
\text { contours }\end{array}$ & $9.68^{*}$ & $0.57^{\dagger}$ & $6(3-9)$ \\
\hline $\begin{array}{l}\text { 3. Visually sharp } \\
\text { reproduction of } \\
\text { the kidneys and } \\
\text { proximal ureters }\end{array}$ & $10.87^{*}$ & $0.92^{*}$ & $8(5-11)$ \\
\hline $\begin{array}{l}\text { 4. Visually sharp } \\
\text { reproduction of } \\
\text { the aorta }\end{array}$ & $10.51^{*}$ & $0.84^{\dagger}$ & $8(4-11)$ \\
\hline $\begin{array}{l}\text { 5. Critically sharp } \\
\text { reproduction of } \\
\text { the gallbladder } \\
\text { wall }\end{array}$ & $12.51^{*}$ & $0.59^{\ddagger}$ & $5(0-9)$ \\
\hline $\begin{array}{l}{ }^{*} P<0.001 \\
{ }^{\dagger} P<0.05 \\
{ }^{*} \text { Not significant }\end{array}$ & & & \\
\hline
\end{tabular}

setting for AEC by adjusting the adaptation strength (comparable to Noise index setting for other manufacturers), based on average patient weight, helps to maintain a desired quantum noise level on the image while improving dose efficiency. The quality reference mAs (Qref mAs) required by the AEC system to adjust the $\mathrm{mA}$ must be defined by the user. The adaptation strength setting adjusts this value for the bigger patients, where dose reduction leads to increase in image noise. The VGR analysis shows a better effect for SAFIRE compared to FBP for the present study population with a BMI $<30$ compared to those with a BMI $\geq 30$. 
Several studies show that low-dose protocols for imaged anatomy, such as CT of the paranasal sinuses and CT colon screening, can accommodate for possible further dose reductions, especially with iterative reconstruction without loss in diagnostic accuracy, due to the high contrast differences in the anatomy studied $(26,27)$. For these images with high contrast, there is a slightly increased tolerance for image noise compared to low-dose abdomen images where contrast differences between the solid organs are small, thus limiting the amount of acceptable image noise without impairing the diagnostic accuracy.

This study has several limitations, one of which is use of a low-dose protocol in comparison to a further $30 \%$ reduction in dose. Another limitation is use of SAFIRE strength 1 which restricted the evaluation of the full potential of the SAFIRE algorithm. In future studies, it would be interesting to assess the effect of higher strength settings, i.e. reconstructions deviating more from standard FBP. Finally, the present study did not assess the appearance of artifacts, which is believed to affect image quality in CT examinations.

In conclusion, SAFIRE iterative reconstruction algorithm improved image quality in low-dose abdominal CT. With a SAFIRE strength setting of 1 , the estimated dose reduction was rather small. The full potential of the algorithm remains unclear.

\section{Conflict of interest: None.}

\section{ACKNOWLEDGEMENTS}

The authors are indebted to the readers Bo Ekerling, Lars-Göran Pettersson, Peter Johansson, and Senija Halilic for help with assessment of the observations and to the radiographers at Vrinnevi Hospital in recruiting the study population. We extend our special thanks to Jonas Nilsson-Althén, our hospital physicist, for his help in planning this study. This paper was presented at the following: Siemens User Club meeting, Norrköping, Sweden (May 11, 2012); Swedish Radiology Week, Gothenburg, Sweden (September 12, 2012); and Research and Development Seminar, Norrköping, Sweden (October 17, 2012).

\section{REFERENCES}

1 Valentin J, ed. Volume 102: ICRP Publication 102: Managing Patient Dose in Multi-Detector Computed Tomography (MDCT). Amsterdam: Elsevier; 2007

2 Goo HW. CT radiation dose optimization and estimation: an update for radiologists. Korean J Radiol 2012;13:1-11

3 Berrington de González A, Mahesh M, Kim K-P, et al. Projected cancer risks from computed tomographic scans performed in the United States in 2007. AMA. Arch Intern Med 2009;169:2071-7

4 Smith-Bindeman R, Lipson J, Marcus R, et al. Radiation dose associated with common computed tomography examinations and the associated lifetime attributable risk for cancer AMA. Arch Intern Med 2009;169:2078-86

5 Pearce MS, Salotti JA, Little MP, et al. Radiation exposure from CT scans in childhood and subsequent risk of leukaemia and brain tumours: a retrospective cohort study. Lancet 2012;380:499-505
6 Paterson A, Frush DP. Dose reduction in paediatric MDCT: general principles. Clin Radiol 2007;62:507-17

7 Sunnergårdh J. Iterative filtered back projection methods for helical cone-beam CT. Linköping studies in Science and Technology, Dissertation nr. 1264. Linköping, Sweden. See http://liu.diva-portal.org/ smash/record.jsf?pid=diva2:232734\&rvn=11

8 Leipsic J, Heilbron BG, Hague C. Iterative reconstruction for coronary CT angiography: finding its way. Int J Cardiovasc Imaging 2012;28:613-20

9 Sagara Y, Hara AK, Pavlicek W, et al. Abdominal CT: Comparison of low-dose CT with adaptive statistic iterative reconstruction and routine dose CT with filtered back projection in 53 patients. Am J Roentgenol 2010;195:713-19CEC

10 Bittencourt MS, Schmidt B, Seltmann M, et al. Iterative reconstruction in image space (IRIS) in cardiac computed tomography: initial experience. Int J Cardiovasc Imaging 2010;27:1081-7

11 Leipsic J, LaBounty TM, Heilbron B, et al. Adaptive Statistical Iterative Reconstruction: Assessment of image noise and image quality in Coronary CT Angiography. Am J Roentgenol 2010;195:649-54

12 Hara AK, Paden RG, Silva AC, et al. Iterative reconstruction technique for reducing body radiation dose at CT: feasibility study. Am J Roentgenol 2009;193:754-71

13 European guidelines on quality criteria for computed tomography. Report EUR 16262 EN. Luxembourg: Office for Official Publications of the European Communities; 1996. See http://www.drs.dk/guidelines/ct/quality/ index.htm

14 Smedby Ö, Fredrikson M. Visual grading regression - analysing data from visual grading experiments with regression models. $\mathrm{Br} J$ Radiol 2010;83:767-75

15 Snijders TAB. Fixed and random effects. In: Everitt BS, Howell DC, eds. Encyclopaedia of Statistics in Behavioural Science. Chichester: Wiley, 2005

16 Smedby Ö, Fredrikson M, De Geer J, et al. Quantifying the potential for dose reduction with visual grading regression. $\mathrm{Br} J$ Radiol 2013;86:31197714

17 Grilli L, Rampichini C. A review of random effects modelling using GLLAMM in Stata. In Software Reviews of Multilevel Analysis Packages. Centre for Multilevel Modelling. Bristol: University of Bristol, UKU, 2005. See http://www.bristol.ac.uk/cmm/learning/mmsoftware/gllamm. html

18 Shah NB, Platt SL. ALARA: is there a cause for alarm? Reducing radiation risks from computed tomography scanning in children. Curr Opin Paediatr 2008;20:243-7

19 Newman B, Callahan MJ. ALARA (as low as reasonably achievable) CT 2011 - executive summary. Pediatr Radiol 2011;41:453-5

20 Mahesh M. Medical radiation exposure with focus on CT. Rev Environ Health 2010;25:69-74

21 Kalra MK, Woisetschläger M, Dahlström N, et al. Radiation dose reduction with sinogram affirmed iterative reconstruction technique for abdominal computed tomography. J Comput Assist Tomogr 2012;36:339-46

22 Winklehner A, Karlo C, Puippe G, et al. Raw data-Based iterative reconstruction in body CTA: evaluation of radiation dose saving potential. Eur Radiol 2011;21:2521-6

23 Moscariello A, Takx RAP, Schoepf J, et al. Coronary CT angiography: Image quality, diagnostic accuracy, and potential for radiation dose reduction using a novel iterative image reconstruction technique comparison with traditional filtered back projection. Eur Radiol 2011;21:2130-8

24 Martinsen ACT, Saether HK, Hol PK, et al. Iterative Reconstruction reduces abdominal CT dose. Eur J Radiol 2011;81:1483-7

25 Nelson RC, Feuerlein S, Boll DT. New iterative reconstruction techniques for cardiovascular computed tomography: How do they work, and what are the advantages and disadvantages? J Cardiovasc Comput Tomogr 2011;5:286-92

26 Flicek KT, Hara AK, Silva AC, et al. Reducing the radiation dose for CT colonography using adaptive statistical iterative reconstruction: a pilot study. Am J Roentgenol 2011;195:126-31

27 Bulla S, Blanke P, Hassepass F, et al. Reducing the radiation dose for low-dose CT of the paranasal sinuses using iterative reconstruction: feasibility and image quality. Eur I Radiol 2011;81:2246-50 

PAPER 



\title{
Assessment of image quality in abdominal CT: potential dose reduction with model-based iterative reconstruction
}

\author{
Bharti Kataria $^{1}$ (D) Jonas Nilsson Althén ${ }^{2}$ - Örjan Smedby ${ }^{3}$ (D) Anders Persson ${ }^{1}$ (D) \\ Hannibal Sökjer ${ }^{4} \cdot$ Michael Sandborg $^{2}$ (D)
}

Received: 28 February 2017 /Revised: 23 August 2017 / Accepted: 29 September 2017 /Published online: 24 January 2018

C The Author(s) 2017. This article is an open access publication

\begin{abstract}
Purpose To estimate potential dose reduction in abdominal CT by visually comparing images reconstructed with filtered back projection (FBP) and strengths of 3 and 5 of a specific MBIR.

Material and methods A dual-source scanner was used to obtain three data sets each for 50 recruited patients with 30 , 70 and $100 \%$ tube loads (mean $\mathrm{CTDI}_{\mathrm{vol}} 1.9,3.4$ and 6.2 $\mathrm{mGy}$ ). Six image criteria were assessed independently by five radiologists. Potential dose reduction was estimated with Visual Grading Regression (VGR).

Results Comparing 30 and $70 \%$ tube load, improved image quality was observed as a significant strong effect of log tube load and reconstruction method with potential dose reduction relative to FBP of $22-47 \%$ for MBIR strength 3 ( $p<0.001$ ). For MBIR strength 5 no dose reduction was possible for image criteria 1 (liver parenchyma), but dose reduction between 34 and $74 \%$ was achieved for other criteria. Interobserver reliability showed agreement of $71-76 \%\left(\kappa_{\mathrm{W}} 0.201-0.286\right)$ and intra-observer reliability of $82-96 \%\left(\kappa_{\mathrm{w}} 0.525-0.783\right)$. Conclusion MBIR showed improved image quality compared to FBP with positive correlation between MBIR
\end{abstract}

Bharti Kataria

Bharti.Kataria@liu.se

1 Department of Radiology, Department of Medical and Health Sciences, Center for Medical Image Science and Visualization (CMIV), Linköping University, S-581 85 Linköping, Sweden

2 Department of Medical Physics, Department of Medical and Health Sciences, Linköping University, Linköping, Sweden

3 School of Technology and Health (STH), KTH Royal Institute, Stockholm, Sweden

4 Department of Medical and Health Sciences, Linköping University, S-581 83 Linköping, Sweden strength and increasing potential dose reduction for all but one image criterion.

Key Points

- MBIR's main advantage is its de-noising properties, which facilitates dose reduction.

- MBIR allows for potential dose reduction in relation to FBP.

- Visual Grading Regression (VGR) produces direct numerical estimates of potential dose reduction.

- MBIR strengths 3 and 5 dose reductions were 22-34 and 34$74 \%$.

- MBIR strength 5 demonstrates inferior performance for liver parenchyma.

Keywords Dose · Computed tomography · Iterative reconstruction $\cdot$ Abdomen $\cdot$ FBP

\begin{tabular}{ll}
\multicolumn{2}{l}{ Abbreviations } \\
ADMIRE & Advanced Modeled Iterative Reconstruction \\
ALARA & As low as reasonably achievable \\
CT & Computed tomography \\
FBP & Filtered back projection \\
IR & Iterative reconstruction \\
kV & Kilovolt \\
MBIR & Model-based iterative reconstruction \\
mAs & Milliampere-second \\
mSv & Millisievert \\
Qref & Quality reference \\
VGR & Visual Grading Regression
\end{tabular}

\section{Introduction}

Technical developments and new applications have led to an increase in the use of computed tomography (CT) in medical 
imaging and the associated population doses that arise from it $[1,2]$. CT contributes up to $70 \%$ of the collective effective dose, although it accounts for only $10-15 \%$ of the total medical imaging procedures that use ionising radiation [3-6]. Multiphase examinations are more common in abdominal CT, and approximately $30 \%$ of CT examinations are abdominal and pelvic, which deliver an effective dose of approximately $6-8 \mathrm{mSv}$ [7]. In recognition of benefits of CT [8], the optimisation of the clinical protocols is motivated to keep the dose as low as reasonably achievable (ALARA principle) [9, 10].

Modern CT equipment presents a number of dosereduction strategies such as automatic tube current modulation, iterative reconstruction algorithms (IR), dynamic collimation and dose efficient detectors, among others $[1,10,11]$.

IR selectively reduces statistical noise in the images thus improving image quality of subtle details, and may facilitate dose reduction. There has been successful improvement in performance of the IR algorithms as they have evolved in the past decade from statistical to model-based algorithms (MBIR) [11, 12]. MBIR, which may be applied at different strengths, perform noise reduction in both raw data and image domains and incorporate physical models to accurately correct for a variety of image degrading effects $[13,14]$. Several studies indicate that an increase in the strength of IR allows for larger dose reductions [13, 15, 16]. Evaluations of radiological imaging methods can be performed either by studying their ability to provide correct diagnoses [17] or by visual assessment of well-defined image quality features (visual grading) [18]. However, to our knowledge, there are no studies that directly estimate the dose-reduction potential of the Advanced Modeled Iterative Reconstruction (ADMIRE, Siemens, Erlangen, Germany) algorithm in the clinical setting using pair-wise comparison of images.

The aim of this study was to assess visual image quality between filtered back projection (FBP) and ADMIRE strengths 3 and 5 (out of 5) in abdominal $\mathrm{CT}$, and to estimate the dose-reduction potential of the reconstruction algorithm.

\section{Material and methods}

This was a regional ethical board-approved prospective study conducted at the Centre for Medical Image Science and Visualization (CMIV), Linköping University, Sweden.

A Somatom Force 192-slice dual source CT (Siemens) was used to obtain three data sets of images per patient at 30,70 and $100 \%$ dose levels from a single abdominal acquisition. These were achieved without additional patient exposure as the 30 and $70 \%$ tube loads were obtained simultaneously using the dual sources. The acquisition parameters from a standard clinical abdominal protocol are presented in Table 1. Due to a $35.5-\mathrm{cm}$ diameter restriction of the small detector scan field of view (SFoV), ethical approval was obtained for 90 patients as anatomical fit to the smaller SFoV could only be determined after the scan was performed.

Patients were informed as to the intent of the study and written consent and approval were obtained. Inclusion criteria were patients over the age of 18 years undergoing a clinical abdominal CT with appropriate patient body habitus determined by visual estimation and use of a calliper to estimate patient size before the scan. Forty patients were excluded due to size and anatomical variations.

Of the 50 examinations, 25 were contrast-enhanced and 25 non-enhanced examinations.

Critical care was taken in patient positioning at isocentre in the gantry. Demographical data such as age, height and weight were also recorded.

\section{Procedure}

The images were anonymised so as to avoid identification of individual patients.

Images at each dose level (30, 70 and $100 \%)$ were reconstructed with FBP and ADMIRE strengths 3 and 5. Pairwise visual grading was carried out independently by five radiologists with varying experience (6-20 years), using four modified criteria (C1-C4) from the European guidelines for image quality in abdominal CT [19] together with image noise (C5) and overall image quality (C6) [20] to suit the purpose of this study. The criteria used were as follows:

Table 1 Acquisition parameters for dual source Somatom Force (Siemens, Erlangen, Germany) for tube A and tube B (smaller detector) and Dual Energy Composition (DE comp) set at 0.5 i.e. equal $\mathrm{kV}$ weighting for each $\mathrm{x}$-ray tube

\begin{tabular}{lccccccccc}
\hline Source & $\mathrm{U}(\mathrm{kV})$ & Qref (mAs) & Acquisition & Rotation (s) & Pitch & Care Dose 4D & Kernel & Dose level & Slice thickness/Increment (mm) \\
\hline Tube A+B & 120 & 140 & $192 \times 0.6$ & 0.5 & 0.6 & Yes & Br36 & $100 \%$ & $3 / 2$ \\
Tube A & 120 & 98 & $192 \times 0.6$ & 0.5 & 0.6 & Yes & Br36 & $70 \%$ & $3 / 2$ \\
Tube B & 120 & 42 & $192 \times 0.6$ & 0.5 & 0.6 & Yes & Br36 & $30 \%$ & $3 / 2$ \\
\hline
\end{tabular}

Qref quality reference 
C1. Visually sharp reproduction of the liver parenchyma $\mathrm{C} 2$. Visually sharp reproduction of the pancreas contour C3. Visually sharp reproduction of the contours of the kidneys and proximal ureters

C4. Visually sharp reproduction of the contours of lymph nodes smaller than $15 \mathrm{~mm}$

C5. Image noise not affecting interpretation

C6. Overall image quality for diagnostic purposes.

All of the radiologists had 3-4 years' of experience with SAFIRE strength 3 and one radiologist has been working with ADMIRE strength 3 for a year.

Prior to the study the participating radiologists were coached in grading the different aspects of subjective image quality so as to form a similar understanding of interpretation of the image criteria in order to minimise inter-observer variation. The data sets used in the coaching session were not included in the study population. Each reader rated the criteria in a randomised, blinded and pair-wise approach on DICOMcalibrated (EIZO RX 240) PACS version 17.3 (Sectra, Linköping, Sweden) workstations. The image pairs were graded on a 5-point Likert-type scale (Table 2).

Comparison of 12 pairs of image stacks (Fig. 1) per patient resulted in $600(12 \times 50)$ image pairs per radiologist and a total of 3,000 $(600 \times 5)$ image pair assessments. Five of the image pair assessments were replicated to calculate the intraobserver reliability.

\section{Statistical analysis}

Image quality scores were statistically analysed using visual grading regression (VGR) [18]. VGR is an ordinal logistic regression method applied to scores from observer ratings, controlling for dependencies between observers, patients, tube loads and reconstruction methods. Statistical analyses were performed with the software Stata 13.1 (Stata Corporation LP, College Station, TX, USA) using the multi-level mixed-effects ordered logistic regression (meologit) command. The regression coefficients describe how the image quality depends on the choice of tube load and reconstruction algorithm, respectively. By relating two of these coefficients to each other, it is possible to

Table 2 Ordinal grading scores used for each image criterion in the visual image quality assessment

Grading scores

-2 image on left monitor is better than image on right monitor

-1 image on left monitor is probably better than image on right monitor 0 images on left and right monitors are equivalent

+1 image on right monitor is probably better than image on left monitor +2 image on right monitor is better than image on left monitor

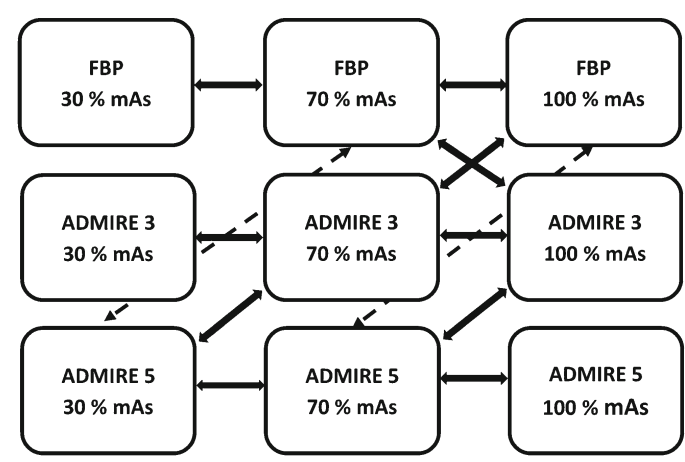

Fig. 1 Schematic diagram of the axial image stacks acquired at tube loads, Qref mAs $42(30 \%), 98(70 \%)$ and $140(100 \%)$, reconstructed using filtered back projection (FBP) and ADMIRE strengths 3 and 5, with arrows showing the pairwise comparisons performed

estimate the potential dose reduction $(D R)$ when replacing one algorithm with another from the equation $D R=1-$ $e^{-(b / a)}$, where $a$ is the regression coefficient for $\log \mathrm{mAs}$ and $b$ that for the iterative reconstruction algorithm [21].

Inter-observer and intra-observer reliabilities were described with the weighted kappa $\left(\kappa_{\mathrm{w}}\right)$ [22] using the kappa 2 command in Stata. The null hypothesis is that neither tube load nor reconstruction method influence perceived image quality. The significance limit was set at $\mathrm{p}=0.05$.

\section{Results}

Of the 50 patients examined, 22 were women, age range 22 90 years (standard deviation (SD) 16.9, mean 64.6) with a body mass index (BMI) of 16.4-27.3 kg/m² (SD 3.0, mean 21.8 ) and 28 men, age range $44-85$ years (SD 10.9, mean 64.8) with a BMI of 16.6-26.2 kg/m $\mathrm{m}^{2}$ (SD 2.1, mean 23.2). For the study population the $\mathrm{CTDI}_{\mathrm{vol}}$ ranged from 3.9 to $9.1 \mathrm{mGy}$ (SD 1.3, mean 6.2 mGy), size-specific dose estimate (SSDE) ranged from 6.3 to $12.8 \mathrm{mGy}$ (SD 1.5, mean 8.6 $\mathrm{mGy}$ ), and dose-length product (DLP) ranged from 161 to 468 mGy.cm (SD 70, mean 292 mGy.cm).

The frequency histograms (Fig. 2) for each criterion show the percentage of favourable versus unfavourable scores (\%) with respect to reconstruction algorithm and dose level. Highest scores are seen for quality reference (Qref) mAs 98 (70\% dose level) for all image criteria. ADMIRE 3 also tended to yield higher scores when compared with FBP and ADMIRE 5. For ADMIRE 5, scores for overall image quality (criterion 6) were equivalent to FBP and inferior to FBP for criterion 1 (liver parenchyma). Surprisingly the scores for full dose images (Qref mAs 140) are lower than those at the 70\% dose level (Qref mAs 98), suggesting that no image quality improvements are obtained with increase in 
a

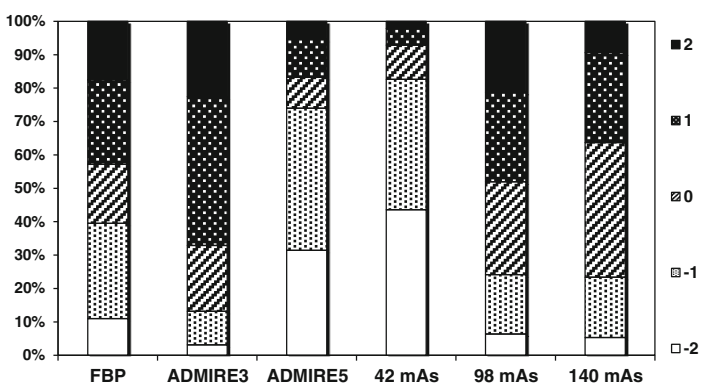

C

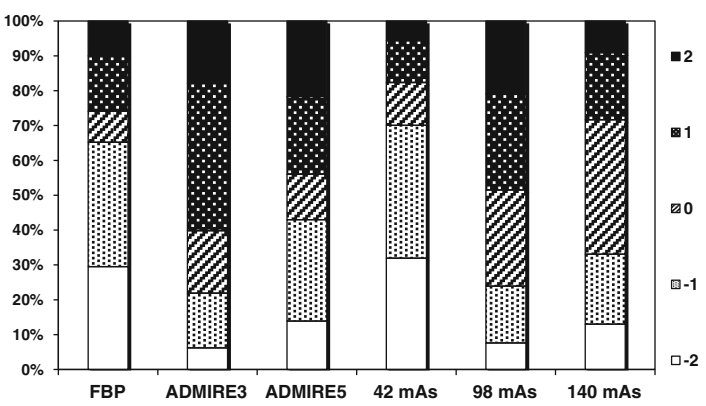

e

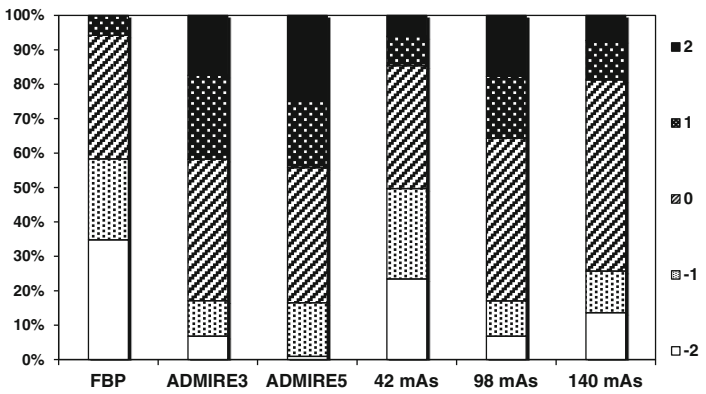

Fig. 2 The bars show the percentage of scores assigned to the current image type when compared to other image types. Score 2 indicates that the current image type was rated as superior to the alternative, score 1 that it was rated as probably superior to the alternative, score 0 that the alternatives were rated as equivalent, score -1 that the current image type was rated as probably inferior to the alternative, and score -2 that it was rated as inferior to the alternative. a (C1) Favourable versus (vs.) unfavourable scores for image quality criterion 1: Visually sharp reproduction of the liver parenchyma. b (C2) Favourable vs. unfavourable scores for image quality criterion 2: Visually sharp b

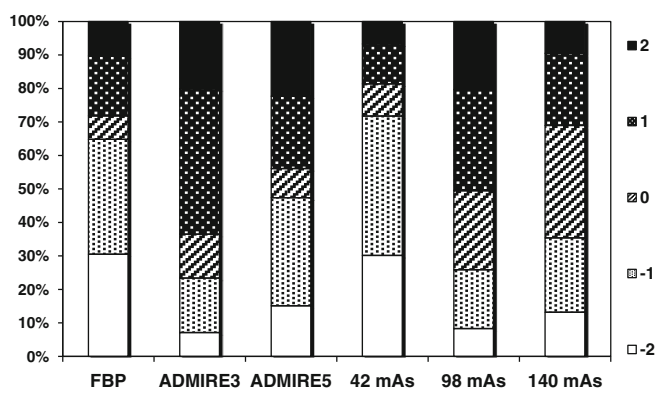

d

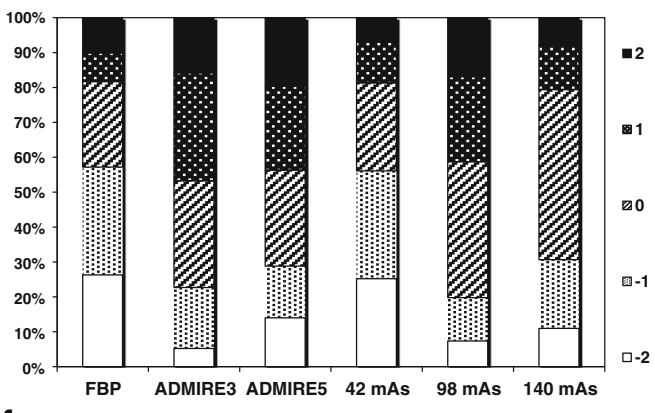

f

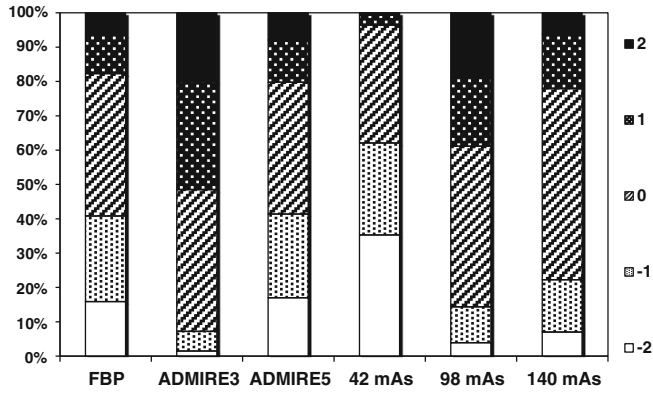

reproduction of pancreas contour. c (C3) Favourable vs. unfavourable scores for image quality criterion 3: Visually sharp reproduction of contours of the kidneys \& proximal ureters. d (C4) Favourable vs. unfavourable scores for image quality criterion 4: Visually sharp reproduction of the contours of the lymph nodes $<15 \mathrm{~mm}$. e (C5) Favourable vs. unfavourable scores for image quality criterion 5: Image noise not affecting interpretation. f (C6) Favourable vs. unfavourable scores for image quality criterion 6: Overall image quality for diagnostic purposes

Table 3. A positive value of the regression coefficient in the table indicates that image quality was improved by the change in Qref mAs. A negative value, on the other hand, indicated a reduction in image quality. For FBP, image quality increases with an increase in tube load. For ADMIRE 3 and ADMIRE dose. Visual demonstration of image quality in one of the study patients obtained with three tube loads and three reconstruction algorithms are presented in Fig. 3.

The effect on image quality of varying the tube load for each reconstruction algorithm separately is presented in 
5 , however, the image quality seems to increase between Qref mAs 42 and 98, but then to decrease for interval Qref mAs 98 and 140. VGR assumes that there is a linear relationship between the $\log \mathrm{mAs}$ and the logit function score. As this assumption does not hold for the interval between Qref $\mathrm{mAs} 98$ and 140, the subsequent VGR statistical analysis is based on Qref mAs interval 42 to 98 .

The VGR result for $\log \mathrm{mAs}$ and ADMIRE 3 and ADMIRE 5 comparing dose levels at Qref mAs 98 with 42 are presented in Table 4. A significant strong effect of $\log \mathrm{mAs}(\mathrm{p}<0.001)$ for all six criteria was noted, which indicated an increase in dose for the same algorithm leads to a corresponding increase in image quality. ADMIRE strength 3 resulted in a strongly significant ( $p$ $<0.001$ ) increase in image quality relative to FBP for all six criteria. This is reflected in the estimated potential dose reduction ranging from $22 \%$ (liver parenchyma) to $47 \%$ (image noise). Strong significant results are also seen for ADMIRE 5, criteria 2-6 with a slightly higher dose reduction ranging from $34 \%$ (overall image quality) to $74 \%$ (image noise), with the exception of criterion 1 (liver parenchyma), for which there was no significant difference between ADMIRE 5 and FBP when assessing the delineation of liver parenchyma.

Comparing results between ADMIRE 3 and ADMIRE 5 in relation to FBP (Table 4), criteria 2-6 suggest that the dose reduction potential is higher with increase in strength. However, for ADMIRE 5 criterion 1 (liver parenchyma) there was no dose reduction possible. Image noise decreases with increase in IR strength, which is reflected by the slightly higher estimated dose reduction for ADMIRE 5 compared to ADMIRE 3.
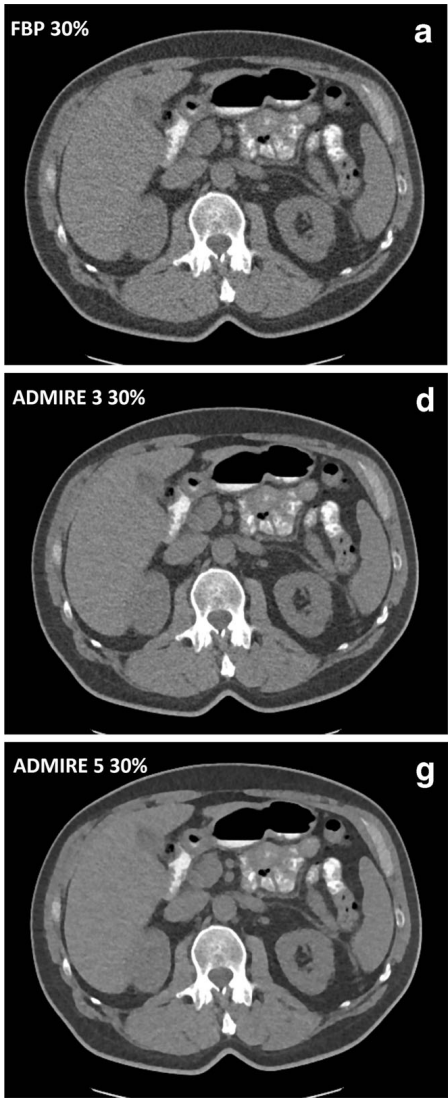

g
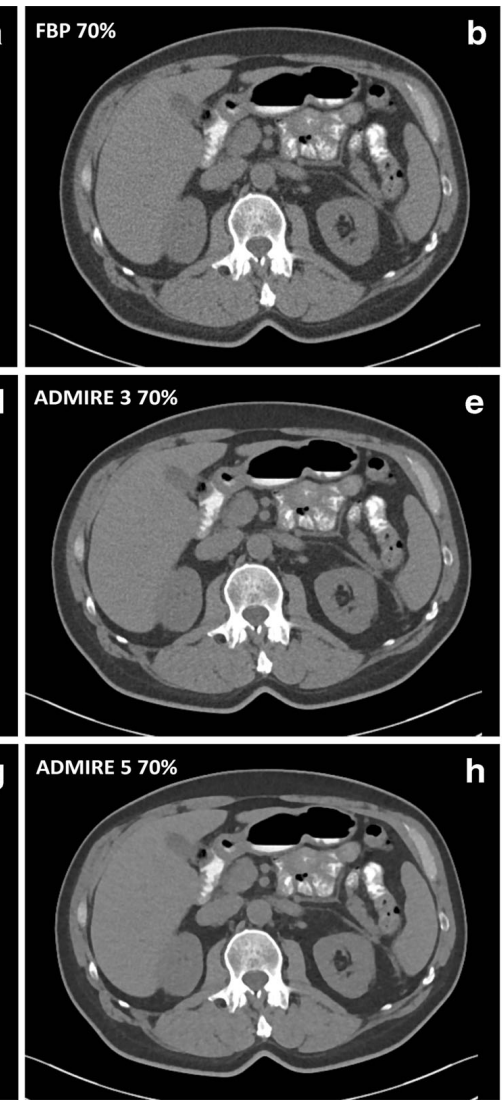

Fig. 3 Transverse CT sections at 30,70 and 100\% tube loads reconstructed with (a) filtered back projection (FBP) $30 \%$, (b) FBP 70\%, (c) FBP 100\%, (d) ADMIRE 3 30\%, (e) ADMIRE 3 70\%, (f) (BMI) of $24.8 \mathrm{~kg} / \mathrm{m}^{2}$ e

h
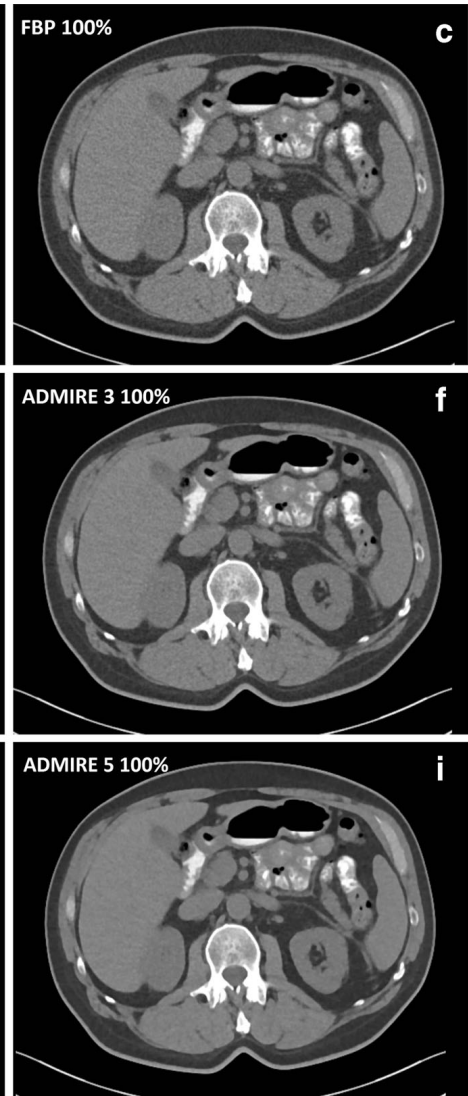

ADMIRE 3 100\%, (g) ADMIRE 5 30\%, (h) ADMIRE $570 \%$ and (i) ADMIRE $5100 \%$ in a 59-year-old male patient with a body mass index 
Table 3 Dependency on tube load for different reconstruction algorithms. Visual grading regression (VGR) coefficients for log (mAs) in pairwise comparisons of two quality reference (Qref) mAs values

\begin{tabular}{|c|c|c|c|c|c|c|}
\hline \multirow[t]{3}{*}{ Criterion } & \multicolumn{6}{|c|}{ Reconstruction algorithm } \\
\hline & \multicolumn{2}{|l|}{ FBP } & \multicolumn{2}{|l|}{ ADMIRE 3} & \multicolumn{2}{|l|}{ ADMIRE 5} \\
\hline & $\begin{array}{l}42 \text { mAs vs. } 98 \\
\text { mAs }\end{array}$ & $\begin{array}{l}98 \mathrm{mAs} \text { vs. } 140 \\
\mathrm{mAs}\end{array}$ & $\begin{array}{l}42 \text { mAs vs. } 98 \\
\text { mAs }\end{array}$ & $\begin{array}{l}98 \mathrm{mAs} \text { vs. } 140 \\
\mathrm{mAs}\end{array}$ & $\begin{array}{l}42 \text { mAs vs. } 98 \\
\text { mAs }\end{array}$ & $\begin{array}{l}98 \mathrm{mAs} \text { vs. } 140 \\
\mathrm{mAs}\end{array}$ \\
\hline 1. Liver parenchyma & - & $3.36 * *$ & $2.20 * * *$ & $-0.28^{\circ}$ & $1.49 *$ & $-0.37^{\circ}$ \\
\hline 2. Pancreatic contours & - & $1.89 * *$ & $2.41 * * *$ & $-0.89^{*}$ & $1.37 * * *$ & $-2.62 * * *$ \\
\hline 3. Kidneys and proximal ureters & $7.25^{*}$ & $2.39 *$ & $2.29 * * *$ & $-0.92 *$ & $1.46 * * *$ & $-2.20 * * *$ \\
\hline $\begin{array}{l}\text { 4. Lymph nodes }<15 \mathrm{~mm} \text { in } \\
\text { diameter }\end{array}$ & $6.50 *$ & $2.09 * *$ & $1.83 * * *$ & $-1.93 * * *$ & $1.14 * * *$ & $-2.20^{*}$ \\
\hline 5. Image noise & $5.49 * *$ & $4.70 * *$ & $3.07 * *$ & - & $4.79 *$ & $1.07^{\circ}$ \\
\hline 6. Overall image quality & - & - & $3.68 *$ & $0.03^{\circ}$ & $3.74 * *$ & $-1.27 * *$ \\
\hline
\end{tabular}

$* \mathrm{p}<0.05, * * \mathrm{p}<0.01, * * * \mathrm{p}<0.001{ }^{\circ}$ not significant

- Convergence was not achieved with the statistical software available

When comparing ADMIRE strength 3 with ADMIRE strength 5 (Table 5), the regression yielded significant $(\mathrm{p}<0.001)$ values for most criteria when dose levels for the same algorithm strength are compared, confirming that ADMIRE 5 is superior to ADMIRE 3. However, for criterion 1 (liver parenchyma) the negative coefficient value $(-0.98)$ indicates that image quality in ADMIRE 5 is significantly lower compared to ADMIRE 3 and hence does not allow for any further dose reduction. Also, overall image quality with a regression coefficient value of -0.85 was not significant. For the criteria assessed, ADMIRE strengths 3 and 5 are superior when compared to FBP individually except for criterion 1 (ADMIRE 5). When it comes to delineation of the liver parenchyma and overall image quality, ADMIRE 5 was inferior to ADMIRE 3 with no dose reduction possible.
There were marginal differences in the results of the VGR analysis between the groups of patients who received intravenous contrast material and those who did not (data not shown).

The inter-observer agreement was fair, $71-76 \%$ with $\kappa_{\mathrm{w}}$ ranging from 0.201 (confidence interval (CI) $0.175-0.228$ ) to 0.286 (CI $0.258-0.314$ ). The intra-observer $\kappa_{\mathrm{w}}$ values ranged from 0.525 (CI $0.209-0.840$ ) to 0.783 (CI $0.577-$ 1.021 ), showing a moderate to substantial agreement between 82 and $96 \%$ for all the criteria.

\section{Discussion}

In radiology, several analysis methods can be used to describe image quality. The receiver operating characteristic (ROC)

Table 4 Visual grading regression (VGR) coefficients for all six criteria with estimated dose reduction values for comparison of images reconstructed with ADMIRE 3, ADMIRE 5 and filtered back projection (FBP) for dose interval between quality references (Qref) mAs 98 and 42

\begin{tabular}{|c|c|c|c|c|c|}
\hline \multirow[t]{2}{*}{ Criterion } & \multicolumn{3}{|c|}{ Regression coefficients } & \multicolumn{2}{|c|}{$\begin{array}{l}\text { Estimated dose reduction } \\
\text { ( } 95 \% \text { confidence limits) }\end{array}$} \\
\hline & $\log (\mathrm{mAs})$ & ADMIRE 3 & ADMIRE 5 & ADMIRE 3 & ADMIRE 5 \\
\hline 1. Liver parenchyma & $2.28 * * *$ & $0.57 * * *$ & $-0.08^{\circ}$ & $\begin{array}{l}22 \% \\
(11 \% ; 33 \%)\end{array}$ & - \\
\hline 2. Pancreatic contours & $2.00 * * *$ & $0.92 * * *$ & $1.73^{* * *}$ & $\begin{array}{l}37 \% \\
(26 \% ; 48 \%)\end{array}$ & $\begin{array}{l}58 \% \\
(53 \% ; 63 \%)\end{array}$ \\
\hline 3. Kidneys and proximal ureters & $2.21 * * *$ & $1.11 * * *$ & $2.09^{* * *}$ & $\begin{array}{l}40 \% \\
(31 \% ; 49 \%)\end{array}$ & $\begin{array}{l}61 \% \\
(57 \% ; 66 \%)\end{array}$ \\
\hline 4. Lymph nodes $<15 \mathrm{~mm}$ in diameter & $1.72 * * *$ & $1.05^{* * *}$ & $1.93^{* * * *}$ & $\begin{array}{l}46 \% \\
(35 \% ; 57 \%)\end{array}$ & $\begin{array}{l}67 \% \\
(63 \% ; 72 \%)\end{array}$ \\
\hline 5. Image noise & $2.38^{* * *}$ & $1.50^{* * * *}$ & $3.16^{* * *}$ & $\begin{array}{l}47 \% \\
(39 \% ; 55 \%)\end{array}$ & $\begin{array}{l}74 \% \\
(71 \% ; 76 \%)\end{array}$ \\
\hline 6. Overall image quality & $2.69^{* * *}$ & $1.06^{* * * *}$ & $1.10^{* * * *}$ & $\begin{array}{l}33 \% \\
(24 \% ; 41 \%)\end{array}$ & $\begin{array}{l}34 \% \\
(27 \% ; 40 \%)\end{array}$ \\
\hline
\end{tabular}

$* * * \mathrm{p}<0.001,{ }^{\circ}$ not significant 
Table 5 Visual grading regression (VGR) coefficients for all six criteria with estimated dose reduction values for comparison between ADMIRE 3 and ADMIRE 5 in the dose interval quality references (Qref) mAs 98 and 42

\begin{tabular}{|c|c|c|c|}
\hline \multirow[t]{2}{*}{ Criterion } & \multicolumn{2}{|c|}{ Regression coefficients } & \multirow{2}{*}{$\begin{array}{l}\text { Estimated dose } \\
\text { reduction } \\
\text { (95\% confidence } \\
\text { limits) }\end{array}$} \\
\hline & $\begin{array}{l}\log \\
(\mathrm{mAs})\end{array}$ & $\begin{array}{l}\text { ADMIRE } 5 \\
\text { Reconstruction }\end{array}$ & \\
\hline 1. Liver parenchyma & $1.88 * * *$ & $-0.98 * * *$ & $\begin{array}{l}-68 \% \\
(-102 \% ;-35 \%)\end{array}$ \\
\hline 2. Pancreatic contours & $1.84 * * *$ & $0.61 * * *$ & $\begin{array}{l}27 \% \\
(18 \% ; 37 \%)\end{array}$ \\
\hline $\begin{array}{l}\text { 3. Kidneys and proximal } \\
\text { ureters }\end{array}$ & $2.06 * * *$ & $0.77 * * *$ & $\begin{array}{l}31 \% \\
(23 \% ; 39 \%)\end{array}$ \\
\hline $\begin{array}{l}\text { 4. Lymph nodes } \\
<15 \mathrm{~mm} \text { in diameter }\end{array}$ & $1.49 * * *$ & $0.68 * * *$ & $\begin{array}{l}37 \% \\
(26 \% ; 47 \%)\end{array}$ \\
\hline 5. Image noise & $2.42^{* * *}$ & $1.66^{* * *}$ & $\begin{array}{l}50 \% \\
(45 \% ; 55 \%)\end{array}$ \\
\hline 6. Overall image quality & $3.18 * * *$ & $-0.85^{\circ}$ & - \\
\hline
\end{tabular}

$* * * \mathrm{p}<0.001,{ }^{\circ}$ not significant

analysis method is used to evaluate and compare diagnostic performance $[17,23]$. When determining potential dose reduction in the optimisation process, visual grading experiments may be useful $[21,24]$. VGR seems to be the only analysis method that produces direct numerical estimates of potential dose reductions for new acquisition, reconstruction and post-processing techniques while image quality is maintained [25].

The present study aimed to estimate the dose reduction potential of ADMIRE strengths 3 and 5 compared with FBP in a standard-dose abdominal CT. It suggests that ADMIRE while preserving image quality allows for a dose reduction relative to FBP of $22-47 \%$ (ADMIRE 3 ) for all criteria assessed, and 34-74\% (ADMIRE 5) for criteria 2-6 with the exception of liver parenchyma visualisation. Similar results are reported by Greffier et al, who studied the performance of SAFIRE strengths 1-5 compared to FBP in two data sets at 30 and $70 \%$ dose levels. They concluded that a $40-60 \%$ reduction in dose is possible [26]. Gordic et al. [27] evaluated both quantitative and qualitative image quality parameters in abdominal CT using ADMIRE. Results from their study showed an improved image quality with lower noise when comparing ADMIRE with FBP, where the amount of noise reduction ( $53 \%$ for ADMIRE 5) could be translated to a reduction in radiation dose (e.g. reduction in effective mAs). However, their study differs from the present study in that they evaluated visibility of only small structures such as small blood vessels, adrenal glands and lymph nodes.
In the present study, the VGR analysis in Table 3 revealed that the 70\% dose level (Qref mAs 98) scores were, for the iterative algorithms, higher than full dose (Qref mAs 140) scores for all image criteria. This was an unexpected finding. For FBP, the results were in agreement with the general rule that image quality increases with increasing tube load. For the iterative reconstruction algorithms, one might speculate that a certain amount of noise is required for the algorithm to work optimally. It should be noted that due to technical advancements the Somatom Force scanner has been found to give better image quality than other Siemens equipment [28]. A possible conclusion is that the $70 \%$ dose level provides images of sufficiently high image quality. Thus, one can optimise the standard clinical abdominal protocol for the Somatom Force using ADMIRE 3 by reducing the tube load from Qref mAs 140 to 98 without changing the strength of the algorithm. However, as demonstrated in Table 5, by replacing ADMIRE 3 with ADMIRE 5, further dose reduction can be achieved for certain aspects of the image quality, but not all.

With ADMIRE strength 5 there is still a problem as non-linear effects of IR lead to smoothing of the anatomical features and a change in appearance of the anatomy in the images [29]. Mieville et al. [30] reported a change in the appearance of the MBIR images. Certain small objects that were not identified on the FBP images were visualised on the low-dose MBIR images. Suboptimal performance when evaluating small or subtle abdominal structures (i.e. common bile duct, adrenal glands and pancreatic duct) was also reported by Padole et al. [31] when comparing FBP with reduced dose MBIR and Adaptive Statistical Iterative Reconstruction (ASIR). This compromise in the visibility of structures could be attributed to the blotchy, pixelated and plasticlike appearance of the images. The liver parenchyma is a low-contrast object and although image quality is improved using MBIR, it is possible that no improvement is seen in detection of low-contrast details as was the case in the phantom study of Euler et al. [32]. Contrary results were presented by Solomon et al. [13] who studied low-contrast detectability using ADMIRE. There is a possibility that assessing low-contrast detectability in vivo is different to phantom images as the task of clinical assessment by a radiologist, who has access to full image data and patient information, is relatively complex compared to the simple task of assessing subtle lesions in a phantom. Solomon et al. [33] used a similar method to the present study to estimate the dose reduction potential of SAFIRE relative to FBP. They also investigated virtual liver lesion detectability in hybrid images. Although their study was simple compared to clinical reality, such experiments do provide valuable information about how different algorithms render the same lesion differently. In the present study, reader 
confidence in determining the visually sharp reproduction of liver parenchyma was lower for ADMIRE 5 than for FBP. Change in image texture due to the denoising properties of the reconstruction algorithm influences the potential dose reduction depending on the diagnostic task [30]. This may explain the slightly lower estimated dose reduction for ADMIRE 3 and the non-significant result for ADMIRE 5 when assessing the liver parenchyma.

On the other hand, anatomical contour assessments were not a problem even though the higher strength images were smoother in appearance. As our readers pointed out during the coaching session, when intra-abdominal fat is present, the delineation of contours is further enhanced. This was true for the assessment of proximal ureters, lymph nodes and pancreas. However, in patients with low BMI, when a smaller amount of intra-abdominal fat is present, the delineation of contours can be difficult [34].

Low kappa values were seen for inter-observer agreement between all five readers. It is not unusual that there is a variation in perception of image quality among radiologists as viewing strategies differ depending on the approach [35]. The intra-observer agreement in the present study was less than $100 \%$, indicating variation in perception for the same reader at different points in time [36].

Future research is indicated as there is some scepticism among radiologists in using higher strengths of IR. This is mostly related to the change in image texture, due to substantial noise reduction, possibly affecting diagnostic confidence. When comparing delineation of structures in different planes, Mieville et al. [30] reported an improvement in detection of small structures in the coronal plane compared to the axial plane. It would be of interest to study if higher strengths of the algorithm could be made more clinically acceptable with a combination of IR and other post-processing methods. This might increase diagnostic confidence in IR images of higher strength and allow for further dose reductions.

The major limitation of our study was exclusion of overweight patients due to size limitations of the small detector, hence the estimated dose reduction is limited to the patients with a BMI of up to $27.3 \mathrm{~kg} / \mathrm{m}^{2}$. Image quality might be inferior as noise increases in overweight patients with a BMI $>28$, possibly leading to less or no dose reduction [24, 37]. Since there was a variation in patient body habitus, not all scans fitted the $35.5-\mathrm{cm}$ diameter. Some of the patient anatomy, mostly in the pelvic region, slightly exceeded this limit, which may have affected the image quality. However, there were no anatomical image criteria present in this region, except for overall image noise and image quality. It is therefore unlikely that this would have affected the results. There are many image acquisition parameters that affect image quality. We have studied only change in tube load (mAs) and image reconstruction. Hence the study protocol differs from the clinical protocol as a fixed $\mathrm{kV}$ was used for both $\mathrm{x}$-ray tubes without automatic adjustment of tube potential (Care $\mathrm{kV}$ ). Visual grading is an easy and inexpensive method to assess image quality. However, it assumes that whenever normal anatomy is sharply reproduced, the same will apply to pathology. The extent to which this assumption is correct is generally not known. As reconstructed images with MBIR strengths 3 and 5 and FBP all have different appearances, it is difficult to perform a true blinded evaluation of subjective criteria [38]. This enhances the need for further research and analysis of objective image quality parameters to support the subjective findings of this study.

\section{Conclusion}

The model-based iterative reconstruction algorithm ADMIRE showed improved image quality compared to FBP. A positive correlation between ADMIRE strength and increasing potential dose reduction was found for the majority, but not all, of the image criteria.

Acknowledgements We are grateful to Siemens for providing us with a research license for configuration of the Somatom Force to obtain three dose levels per patient. We acknowledge CMIV for providing the research facilities. Håkan Gustafsson is acknowledged for supporting our project and our participating radiologists, Anki Pozson, Johan Asplund, Jenny Öman, Senija Halilic and Peter Johansson, for grading the images. The study was presented both as an oral and an E-poster at the European Congress of Radiology 2017 in Vienna.

\section{Compliance with ethical standards}

Guarantor The scientific guarantor of this publication is Prof. Michael Sandborg.

Conflict of interest The authors of this manuscript declare no relationships with any companies whose products or services may be related to the subject matter of the article.

Funding This work was supported by ALF- and LFoU-grants from Region Östergötland and the Medical Faculty at Linköping University.

Statistics and biometry One of the authors has adequate statistical expertise.

Informed consent Written informed consent was obtained from all subjects (patients) in this study.

Ethical approval Regional Ethical Review Board approval was obtained.

\author{
Methodology \\ - prospective \\ - cross-sectional study \\ - performed at one institution
}


Open Access This article is distributed under the terms of the Creative Commons Attribution 4.0 International License (http:// creativecommons.org/licenses/by/4.0/), which permits unrestricted use, distribution, and reproduction in any medium, provided you give appropriate credit to the original author(s) and the source, provide a link to the Creative Commons license, and indicate if changes were made.

\section{References}

1. Mahesh M (2009) MDCT physics the basics technology, image quality and radiation dose. Lippincott Williams \& Wilkins, Philadelphia

2. Moores M (2017) A review of the fundamental principles of radiation protection when applied to the patient in diagnostic radiology. Radiat Prot Dosim 175:1-9

3. Sun Z, Ng KA, Sarji SA (2010) Is utilisation of computed tomography justified in clinical practice? Part IV: applications of paediatric computed tomography. Singapore Med J 51:457-463

4. Report UNSCEAR (2008) Sources and effects of ionizing radiation. United Nations Scientific Committee on the effects of ionizing radiation. Volume 1: Sources. Report to the general assembly Scientific annexes A \& B

5. Le Coultre R, Bize J, Champendal M et al (2016) Exposure of the Swiss population by radiodiagnostics: 2013 review. Radiat Prot Dosim 169:221-224

6. European Commission (2014) Radiation protection No. 180: Medical radiation exposure of the European Population Part 1/2. Luxembourg, 2014 Available via https://ec.europa.eu/energy/sites/ ener/files/documents/RP180.pdf. Accessed 30th July 2017

7. Power SP, Maloney F, Twomey M, James K, O’Connor OJ, Maher MM (2016) Computed tomography and patient risks: facts, perceptions and uncertainties. World J Radiol 8:902-915

8. Mayo-Smith WW, Hara AK, Mahesh M, Sahani DV, Pavlicek W (2014) How I do it: managing radiation dose in CT. Radiology 273: 657-672

9. Smith-Bindeman R, Lipson J, Markus R et al (2009) Radiation dose associated with common Computed Tomography examinations and the associated lifetime attributable risk for cancer. Arch Intern Med 169:2078-2086

10. Kalra MK, Sodickson AD, Mayo-Smith WW (2015) CT radiation key concepts for gentle and wise use. Radiographics 35:1706-1721

11. Liu L (2014) Model based Iterative Reconstruction: a promising algorithm for today's Computed Tomography Imaging. J Med Radiat Sci 45:131-136

12. Beister M, Kolditz D, Kalender WA (2012) Iterative reconstruction methods in X-ray CT. Phys Medica 28:94-108

13. Solomon J, Mileto A, Ramirez-Giraldo JC, Samei E (2015) Diagnostic Performance of an Advanced Modeled Iterative Reconstruction Algorithm for low-contrast detectability with a third generation Multidetector dual source CT Scanner. Radiology 275: 735-745

14. Nuyts J, De Man B, Fessler JA, Zbijewski W, Beekman FJ (2013) Modelling the physics in iterative reconstruction for transmission computed tomography. Phys Med Biol 58:R63-R96

15. Ott JG, Ba A, Racine D et al (2016) Patient exposure optimisation through task-based assessment of a new model-based iterative reconstruction technique. Radiat Prot Dosim 169:68-72

16. Patino M, Fuentes JM, Singh S, Hahn PF, Sahani DV (2015) Iterative reconstruction techniques in abdominopelvic CT: technical concepts and clinical implementation. AJR Am J Roentgenol 205:W19-W31
17. Park SH, Goo JM, Jo C-H (2004) Receiver operating characteristics (ROC) curve: a practical review for radiologists. Korean J Radiol 5:11-18

18. Smedby Ö, Fredrikson M (2010) Visual grading regression analysing data from visual grading experiments with regression models. Br J Radiol 83:767-775

19. Report EUR 16262 EN (1996) European guidelines on quality criteria for computed tomography. Office for Official Publications of the European Communities. Available via http://www.drs.dk/ guidelines/ct/quality/htmlindex.htm. Accessed 15th Oct 2013

20. Borgen L, Kalra MK, Laerum F et al (2012) Application of adaptive non-linear $2 \mathrm{D}$ and $3 \mathrm{D}$ post processing filters for reduced dose abdominal CT. Acta Radiol 53:335-34219

21. Smedby Ö, Fredrikson M, De Geer J, Borgen L, Sandborg M (2013) Quantifying the potential for dose reduction with visual grading regression. Br J Radiol 86:1-6

22. Abraira V, Pérez de Vargas A (1999) Generalization of the Kappa coefficient for ordinal categorical data, multiple observers and incomplete designs. Qüestiió 23:561-571

23. Metz CE (1986) ROC methodology in radiologic imaging. Invest Radiol 21:720-733

24. Kataria B, Smedby Ö (2013) Patient dose and image quality in a low-dose abdominal CT: a comparison between iterative reconstruction and filtered back projection. Acta Radiol 54:540-548

25. Saffari SE, Löve Á, Fredrikson M, Smedby Ö (2015) Regression models for analyzing radiological visual grading studies - an empirical comparison. BMC Med Imaging 15:1-10

26. Greffier J, Fernandez A, Macri F, Freitag C, Metge L, Beregi JP (2013) Which dose for what image? Iterative reconstruction for CT scan. Diagn Interv Imaging 94:1117-1121

27. Gordic S, Desbiolles L, Stolzmann P et al (2014) Advanced modelled iterative reconstruction for abdominal CT: qualitative and quantitative evaluation. Clin Radiol 69:497-504

28. Wichmann JL, Hardie AD, Schoepf JU et al (2016) Single- and dual-energy CT of the abdomen: comparison of radiation dose and image quality of 2 nd and 3 rd generation dual-source CT. Eur Radiol 27:642-650

29. Larsson J, Båth M, Ledenius K, Caisander H, Thilander-Klang A (2016) Assessment of clinical image quality in paediatric abdominal CT examinations: dependency on the level of adaptive statistical iterative reconstruction (ASiR) and the type of convolution kernel. Radiat Prot Dosimetry 169:123-129

30. Mieville FA, Berteloot L, Grandjean A et al (2013) Model-based iterative reconstruction in Pediatric chest CT: assessment of image quality in a prospective study of children with cystic fibrosis. Pediatr Radiol 43:558-567

31. Padole A, Singh S, Lira D et al (2015) Assessment of filtered back projection, adaptive statistical, and model-based iterative reconstruction for reduced dose abdominal computed tomography. J Comput Assist Tomogr 39:462-467

32. Euler A, Stieltjes B, Szucs-Farkas Z et al. (2017) Impact of modelbased iterative reconstruction on low-contrast lesion detection and image quality in abdominal CT: a 12-reader-based comparative phantom study with filtered back projection at different tube voltages. Eur Radiol. https://doi.org/10.1007/s00330-017-4825-9

33. Solomon J, Marin D, Roy Choudhury K, Patel B, Samei E (2017) Effect of radiation dose reduction and reconstruction algorithm on Image noise, contrast, resolution, and detectability of subtle hypoattenuating liver lesions at multidetector CT: filtered back projection versus a commercial model-based iterative reconstruction algorithm. Radiology. https://doi.org/10.1148/radiol.2017161736

34. Sagara Y, Hara AK, Pavlicek W, Silva AC, Paden RG, Wu Q (2010) Abdominal CT: comparison of low-dose CT with adaptive statistic iterative reconstruction and routine dose CT with filtered back projection in 53 patients. AJR Am J Roentgenol 195:713-719 
35. Drew T, Vo MHL, Olwal A, Jacobson F, Seltzer SE, Wolfe J (2013) Scanners and drillers: characterizing expert visual search through volumetric images. J Vis 13:1-13

36. Birkelo CC, Chamberlain WE, Phelps PS, Schools PE, Zacks D, Yerushalmy J (1947) Tuberculosis case finding. JAMA 133:359366

37. Chang W, Lee JM, Lee K et al (2013) Assessment of a modelbased, iterative reconstruction algorithm (MBIR) regarding image quality and dose reduction in liver computed tomography. Invest Radiol 48:598-606

38. Hérin E, Gardavaud F, Chiaradia M et al (2015) Use of modelbased iterative reconstruction (MBIR) in reduced dose CT for routine follow-up of patients with malignant lymphoma: dose savings, image quality \& phantom study. Eur Radiol 25:2362-2370 
PAPER III 



\title{
Image quality and pathology assessment in CT Urography: when is the low-dose series sufficient?
}

Bharti Kataria ${ }^{1,2,3^{*}}$, Jonas Nilsson Althén ${ }^{2,4}$, Örjan Smedby ${ }^{5}$, Anders Persson ${ }^{1,2,3}$, Hannibal Sökjer $^{2}$ and Michael Sandborg $2,3,4$

\begin{abstract}
Background: Our aim was to compare CT images from native, nephrographic and excretory phases using image quality criteria as well as the detection of positive pathological findings in $\mathrm{CT}$ Urography, to explore if the radiation burden to the younger group of patients or patients with negative outcomes can be reduced.

Methods: This is a retrospective study of 40 patients who underwent a CT Urography examination on a 192-slice dual source scanner. Image quality was assessed for four specific renal image criteria from the European guidelines, together with pathological assessment in three categories: renal, other abdominal, and incidental findings without clinical significance. Each phase was assessed individually by three radiologists with varying experience using a graded scale. Certainty scores were derived based on the graded assessments. Statistical analysis was performed using visual grading regression (VGR). The limit for significance was set at $p=0.05$.
\end{abstract}

Results: For visual reproduction of the renal parenchyma and renal arteries, the image quality was judged better for the nephrogram phase $(p<0.001)$, whereas renal pelvis/calyces and proximal ureters were better reproduced in the excretory phase compared to the native phase $(p<0.001)$. Similarly, significantly higher certainty scores were obtained in the nephrogram phase for renal parenchyma and renal arteries, but in the excretory phase for renal pelvis/calyxes and proximal ureters. Assessment of pathology in the three categories showed no statistically significant differences between the three phases. Certainty scores for assessment of pathology, however, showed a significantly higher certainty for renal pathology when comparing the native phase to nephrogram and excretory phase and a significantly higher score for nephrographic phase but only for incidental findings.

Conclusion: Visualisation of renal anatomy was as expected with each post-contrast phase showing favourable scores compared to the native phase. No statistically significant differences in the assessment of pathology were found between the three phases. The low-dose CT (LDCT) seems to be sufficient in differentiating between normal and pathological examinations. To reduce the radiation burden in certain patient groups, the LDCT could be considered a suitable alternative as a first line imaging method. However, radiologists should be aware of its limitations.

Keywords: Computed tomography, Urography, Low-dose, Optimization, Image quality, Dose

\footnotetext{
*Correspondence: Bharti.Kataria@liu.se

'Department of Radiology, Linköping University, Linköping, Sweden

²Department of Medical \& Health Sciences, Linköping University, Linköping,

Sweden

Full list of author information is available at the end of the article
}

(c) The Author(s). 2019 Open Access This article is distributed under the terms of the Creative Commons Attribution 4.0 International License (http://creativecommons.org/licenses/by/4.0/), which permits unrestricted use, distribution, and

reproduction in any medium, provided you give appropriate credit to the original author(s) and the source, provide a link to the Creative Commons license, and indicate if changes were made. The Creative Commons Public Domain Dedication waiver (http://creativecommons.org/publicdomain/zero/1.0/) applies to the data made available in this article, unless otherwise stated. 


\section{Background}

CT Urography (CTU) has emerged as the modality of choice in imaging of the abdomen in patients with urinary tract diseases due to its high sensitivity and specificity [1]. Although it comes with a high radiation dose penalty, the benefits of $\mathrm{CT}$ imaging outweigh the risk for many of these patients. Optimisation is not only about patient dose and image quality but also about the diagnostic task at hand, i.e. the correct examination technique for a specific diagnostic enquiry in accordance to the ALARA (radiation dose as low as reasonably achievable) and AHARA (image quality as high as reasonably achievable) principles [2, 3]. Patients presented with haematuria or acute flank pain usually undergo diagnostic imaging to rule out any serious conditions underlining upper urinary tract disease such as urolithiasis, renal cell cancer or upper urinary tract urothelial cell carcinoma (UUT-UCC) [4]. Urolithiasis is a common health problem with a high recurrent rate requiring considerable radiological imaging resources for this population, many of which are younger than 50 years of age [5].

The standardized care pathway (SCP) led to general recommendations of the use of medical imaging in diagnostics of urinary tract disease for patients with macroscopic hematuria who are $\geq 40$ years (revised to $\geq 50$ years in 2018), but even younger patients with risk factors are investigated [6]. The majority of the SCP population consist of malignant cancer diagnoses of the urinary tract and a third of the patients present with benign causes of hematuria [6]. Approximately 20-30\% of these patients with symptoms of visible blood have negative outcomes and are being subjected to diagnostic imaging tests and radiation related risks based on the presence of macro-hematuria [6].

Macroscopic hematuria is a common symptom in other treatable benign diseases such as urinary tract infection and targets younger women of child bearing age, among others [7].

CTU is a multiphase examination associated with a relatively high radiation dose and can be justified as a first line investigation in hematuria patients if important risk factors are present and clinical tests indicate high risk probability of cancer [8]. In the emergency department, where the short examination times as well as the detection of alternative diagnoses is paramount, CT is the preferred modality with a selection of scan protocols depending on the indication. The detrimental effects of ionizing radiation and the expanding use of CT technique have been the driving factors in optimization of clinical practices regarding appropriateness criteria and dose reduction [9]. These can be achieved foremost by selective use of nonradiative modalities such as ultrasound and magnetic resonance imaging (MRI) and referrals based on proper clinical indications, preliminary tests and patient groups. Dose reduction is achieved in a number of ways, such as, limiting scan lengths and number of phases, and the use of lower exposure settings. Dose reduction is additionally seen in the use of features such as automatic dose modulation, iterative reconstruction [10-12] and split-bolus techniques especially in diagnostic imaging of younger, high risk patients $[13,14]$. Recent advancements show a trend towards use of low-dose CT (LDCT) in several diagnostic indications such as the investigation of acute flank pain and acute abdomen for diverticulitis, appendicitis and renal stone disease $[15,16]$.

Both the Bonn call for action and the triple AAA campaign were introduced to strengthen the need for stringent measures in radiation protection for safe and appropriate use of ionizing radiation in medical imaging [17-19]. Published literature on renal stone evaluation have validated the trend towards use of low-dose CT (LDCT) due to the high contrast between urinary stones and the surrounding soft tissue $[15,20]$ as well as in investigation of acute abdomen [16]. However, implementation of the LDCT protocol in the clinical setting has been very slow partly due to the low quality of the images and lack of confidence in interpreting reduced-dose images [21]. But with practice and growing experience it is possible to increase diagnostic confidence and acceptance of lower quality images [22].

The diagnostic performance of non-enhanced CT compared to intravenous urography (IVU) [23] and plain abdominal radiography [24] has been evaluated but there are, to our knowledge, no studies that have compared the image quality and pathology assessment between phases as the present study.

The aim was to compare CT images from native, nephrographic and excretory phases using image quality criteria as well as the detection of positive pathological findings in CT Urography to explore if the radiation burden to the younger group of patients, patients undergoing repetitive imaging or patients with negative outcomes can be reduced.

\section{Materials \& methods}

This is a retrospective study approved by the regional ethical board. Of the 50 patients referred for a clinical CTU between 2016-03-14 and 2016-11-22 and examined on a 192-slice dual source scanner in single source mode (Siemens Healthineers, Erlangen, Germany), forty patients were included in the study. The acquisition data are presented in Table 1.

Ten patients were excluded due to motion artefacts, difference in scan protocol and scan range. The standard CTU protocol was used with intravenous administration of contrast medium, Iopromide (Ultravist $370 \mathrm{mgI} / \mathrm{ml}$, Bayer, Dublin, Ireland), the rate and dose tailored to patient body weight using OMNIVIS calculator (GE healthcare). Computed Tomography Dose Index $\left(\mathrm{CTDI}_{\mathrm{vol}}\right)$ and, 
Table 1 Acquisition parameters for a clinical CT Urography on a 192-slice dual source scanner in a single source scan mode

\begin{tabular}{|c|c|c|c|c|c|c|c|c|}
\hline Phase & Acquisition data & $\mathrm{kV}$ & Qref mAs & Gantry Rotation (s) & Pitch & Slice thickness/increment $\mathrm{mm}$ & Scan Delay & Care kV \\
\hline Native & $192 \times 0.6$ & 120 & 45 & 0.5 & 0.6 & $3 / 2$ & & On \\
\hline Nephrogram & $192 \times 0.6$ & 120 & 140 & 0.5 & 0.6 & $3 / 2$ & $100 \mathrm{~s}$ & Semi \\
\hline Excretory & $192 \times 0.6$ & 120 & 45 & 0.5 & 0.6 & $3 / 2$ & $10 \mathrm{~min}$ & On \\
\hline
\end{tabular}

The scan length ranged from diaphragm to symphysis pubis. The images were reconstructed with kernel Bf36 and iterative reconstruction ADMIRE strength 3 . In the nephrographic phase semi Care kV setting was used resulting in $100 \mathrm{kV}$ and effective tube load of $181 \mathrm{mAs}$

Dose Length Product (DLP) were recorded and Size Specific Dose Estimate (SSDE) was calculated based on the antero-posterior (AP) and lateral (LAT) dimensions of each patient at the level of the kidneys using the center slice approach as described in Boos et al. [25, 26].

The standard protocol for CTU consists of three phases: the native or unenhanced phase (low-dose), a nephrographic phase (standard dose) and an excretory phase (low-dose), all of which are reconstructed with multi-planar reconstruction (MPR) in three planes: axial, coronal and sagittal (Fig. 1). In patients $<50$ years of age, the nephrographic phase is limited to the upper abdomen. Patients $>50$ years of age are examined with all three phases from dome of diaphragm to symphysis pubis arch.

During the reading sessions the readers were asked to grade four anatomical structures (renal parenchyma, renal pelvis and calyxes, proximal ureters and renal arteries) obtained from European guidelines for quality criteria on a five-point Likert scale with numerical scores from one to five allocated to response alternatives; criterion was fulfilled, criterion was probably fulfilled, indecisive, criterion was probably not fulfilled and criterion was not fulfilled (Table 2).

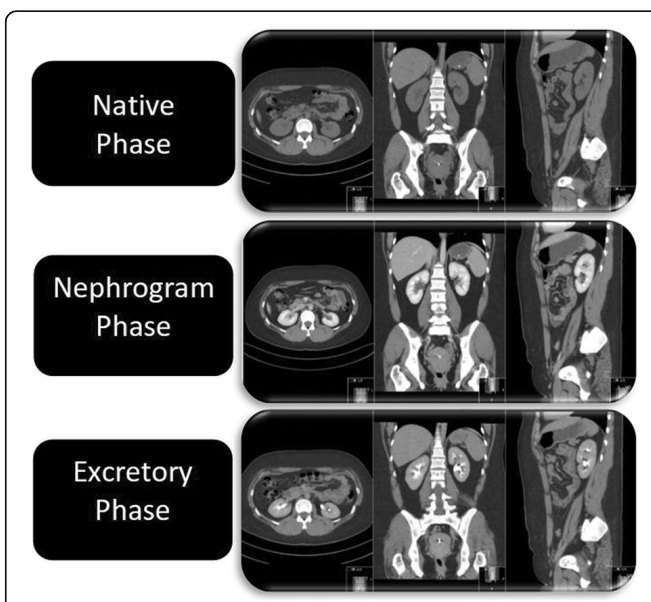

Fig. 1 Illustration of compared phases reconstructed with multiplanar reconstruction (MPR) in three planes. The images are from a $C T$ Urography examination of a study patient
The readers were also asked to assess the presence of pathology in three categories; renal, abdominal and incidental findings. These were also graded on a five-point Likert-type scale based on numerical scores from one to five with response alternatives; normal examination, probably normal examination, indecisive, probably pathological examination and pathological examination (Table 3). To determine how certain the readers were in their scoring of the image criteria and pathological findings, variation in certainty scores between the three phases were also calculated by grouping the score options as shown in Table 4.

Statistical analysis within the Visual Grading Regression (VGR) framework [27] was performed with the software Stata 13.1 (Stata Corporation LP, College Station, TX, USA) using the multi-level mixed-effects ordered logistic regression (meologit) command for image quality scores [28]. Thus, ordinal logistic regression was applied to scores from observer ratings whilst controlling for dependencies between observers and patients, where the regression coefficients describe the combined effect of dose and intravenous contrast on image quality, related to each of the phases of the CTU. The certainty scores were analyzed using the same statistic model as the original scores.

\section{Results}

Of the 40 patients included in the study, 12 were women, age range 27-74 years (mean 58.9 \pm 14 (SD))

Table 2 Anatomical image criteria assessed for each phase including axial, coronal and sagittal multiplanar reconstruction (MPR) planes and graded on a 5-point Likert type scale by allocating a score of 1 to 5

Image Criteria
C1: Visually sharp reproduction of the renal parenchyma
C2: Visually sharp reproduction of the renal pelvis and calyxes
C3: Visually sharp reproduction of the proximal part of the ureters
C4: Visually sharp reproduction of the renal arteries
Grading Scale scores
1: Criterion was fulfilled
2: Criterion was probably fulfilled
3: Indecisive
4: Criterion was probably not fulfilled
5: Criterion was not fulfilled


Table 3 Pathology categories were assessed for each phase including axial, coronal and sagittal multiplanar reconstruction (MPR) planes and graded on a 5-point Likert type scale by allocating a score of 1 to 5

Pathology categories
C5: Pathology in the kidneys and urinary tract related to abdominal
symptoms
C6: Other pathology related to abdominal symptoms
C7: Incidental findings without clinical significance
Grading Scale scores
1: Normal examination
2: Probably normal examination
3: Inconclusive examination
4: Probably pathological examination
5: Pathological examination

with a body mass index (BMI) of $20.7-35.7 \mathrm{~kg} / \mathrm{m}^{2}$ (mean $27.7 \pm 5.0$ ) and 28 men, age range $29-85$ years (mean $63.8 \pm 15.0$ ) with a BMI of $19.3-38.9 \mathrm{~kg} / \mathrm{m}^{2}$ (mean $27.0 \pm 3.7$ ). The mean values, SD and ranges of DLP, $\mathrm{CTDI}_{\mathrm{vol}}, \mathrm{SSDE}$ for the native, nephrogram and excretory phases are displayed in Table 5.

\section{Assessment of anatomical image criteria}

When comparing the native phase with nephrogram and excretory phase respectively, criteria $\mathrm{C} 1$ and $\mathrm{C} 4$ (renal parenchyma and renal arteries) were better reproduced in the nephrographic phase, whereas criteria $\mathrm{C} 2$ and $\mathrm{C} 3$ (renal pelvis/calyxes, proximal ureters), were visually better reproduced in the excretory phase, $(p<0.001)$. Similar results were obtained when comparing the nephrogram to excretory phase, where the nephrographic phase was favorable for criteria $\mathrm{C} 1$ (renal parenchyma) and C4 (renal arteries) $(p<0.001)$. For the delineation of criteria C2 (renal pelvis/calyxes) and C3 (proximal ureters), the excretory phase was preferred ( $p<0.001$ and $p=0.034$, respectively) (Fig. 2).

Table 4 Certainty scores obtained by grouping the assessment scores for anatomical criteria and pathology

\begin{tabular}{ll}
\hline \multicolumn{3}{c}{ Grouping of scores } \\
\hline Certainty score: Image criteria \\
High & 1 and 5 (was fulfilled/not fulfilled) \\
Medium & 2 and 4 (probably fulfilled/probably not fulfilled) \\
Low & 3 (indecisive) \\
Certainty score: & Pathology \\
High & 1 and 5 (normal examination/pathological examination) \\
Medium & 2 and 4 (probably normal examination/probably \\
& pathological examination) \\
\hline
\end{tabular}

Table 5 Distribution of ranges, standard deviation (SD) and mean values of dose length product (DLP), Computed Tomography Dose Index (CTDI $\left.{ }_{\text {vol }}\right)$ and size specific dose estimate (SSDE)

\begin{tabular}{lrrr}
\hline Phase & \multicolumn{1}{c}{$\begin{array}{l}\text { DLP } \\
\text { mGy cm }\end{array}$} & \multicolumn{1}{c}{$\begin{array}{c}\text { CTDIvol } \\
\text { mGy }\end{array}$} & \multicolumn{1}{c}{$\begin{array}{c}\text { SSDE } \\
\text { mGy }\end{array}$} \\
\hline Native & & & 3.51 \\
Mean & 135.7 & 2.84 & 0.79 \\
SD & 38.3 & 0.73 & $2.28-6.93$ \\
Range & $63.8-257.6$ & $1.45-5.39$ & \\
Nephrogram & & & 8.52 \\
Mean & 336.2 & 6.9 & 2.55 \\
SD & 100.6 & 1.9 & $5.45-18.75$ \\
Range & $151.5-696.8$ & $3.46-14.58$ & 3.49 \\
Excretory & & & 0.79 \\
Mean & 135.4 & 2.83 & $2.29-6.84$ \\
SD & 38.7 & 0.73 & \\
Range & $65.4-254.0$ & $1.49-5.32$ & \\
\hline
\end{tabular}

\section{Assessment of pathology}

For the detection of renal and other abdominal pathologies, only marginal and not statistically significant differences in scores were found when comparing the native phase with nephrographic and excretory phases. Similarly, the scores for incidental findings in all three phases were not significantly different. For all three pathology categories, the number of inconclusive scores were very low suggesting that normality and pathology could be assessed in all three phases with marginal differences in scores. The native phase had a higher number of probably normal examination scores compared to the other two phases. None of these differences were statistically significant.

\section{Certainty scores \\ Image criteria}

High certainty scores are seen for criteria C1 (renal parenchyma) and C4 (renal arteries) in favor of the nephrographic phase, when comparing native to nephrographic phase $(p<0.001)$. Similar results are seen for comparison between nephrographic and excretory phase with significant differences in favor of the nephrographic phase $(p<0.001)$. However, when comparing native and excretory phases, certainty for criterion C1 (renal parenchyma) was statistically significantly higher for the native phase whereas no significant difference was found for criterion C4 (renal arteries). Criteria C2 (renal pelvis/calyxes) and C3 (proximal ureters) showed significantly higher certainty scores in the contrast-enhanced phases when comparing native phase to nephrographic and excretory phases $(p<0.001)$. Comparisons between 


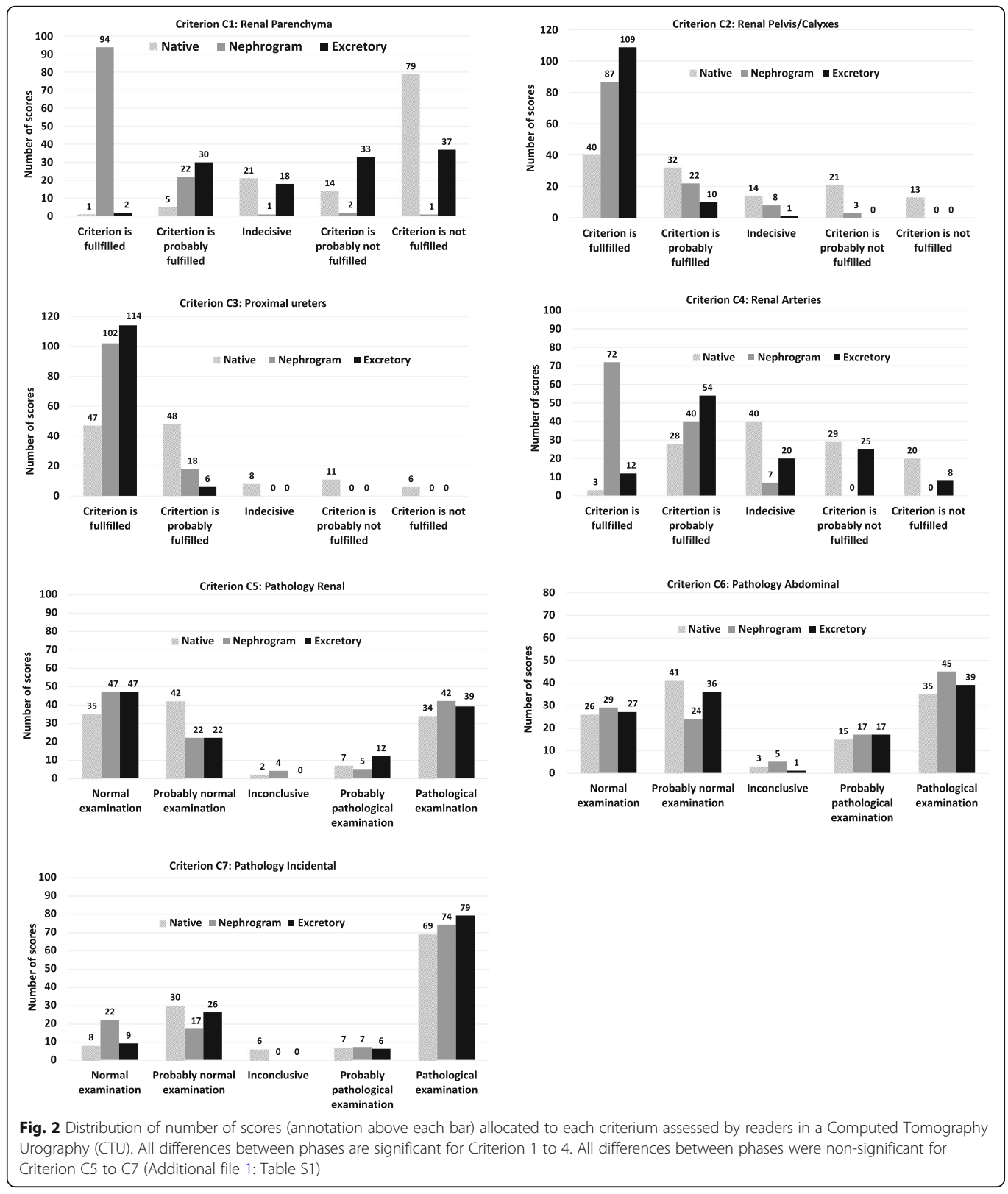

nephrographic and excretory phases show a highly significant difference in scores in favor of excretory phase for criterion C2 (renal pelvis/calyxes) $(p<0.001)$ and a significant difference in scores for criterion $\mathrm{C} 3$ (proximal ureters) $(p=0.021)$.

\section{Pathology}

Significantly high certainty scores in favor of the contrast enhanced phases are obtained for C5 (renal pathology) $(p<0.05)$, when comparing native to nephrographic and excretory phases, however, there 
was no significant difference between the two contrast-enhanced phases. Marginal not significant differences in medium and high certainty scores are observed for Criteria C6 (abdominal pathology) and C7 (incidental findings) when comparing all three phases with each other. Except for determination of C7 (incidental findings), which was statistically significant in favor of the nephrographic phase $(p<0.01)$, when native and nephrographic phases were compared, all other differences in scores between phases were not significant.

\section{Discussion}

The present study showed that the contrast-enhanced phases were considered significantly better for determination of renal pathology. This is not an unusual finding as the acquisition delays after contrast injection are designed to render anatomical features in the best possible way. However, the marginal differences in scores when assessing pathology in the three categories were not significant, with very few inconclusive scores suggesting that it was possible to determine whether the examination was normal or pathological in all three phases. This is also demonstrated by the larger number of high and medium certainty scores for all three categories (Fig. 3). The LDCT is one of many alternatives, that could possibly be used to differentiate between normal and pathological in order to reduce the radiation burden in patients with negative outcomes and those who are more sensitive to ionising radiation.

There are studies that have evaluated the effect of iterative reconstruction on dose reduction in patients presented with acute abdominal pain $[11,12]$. Lee et al. [12] concluded that despite subjective differences in image quality between full-dose and half-dose images, the diagnostic performance is maintained for the lowdose images with the exception of lesion detection at sub-centimeter size. Poletti et al. [11] concluded that low-dose imaging can be achieved in non-obese patients. However, these studies were performed with contrast enhancement which, combined with dose reduction properties of IR, maintains the diagnostic performance of the low-dose protocol when compared to contrast-enhanced standard-dose protocol.

In order to optimize the use of CT in urinary tract investigations, there are several methods that can be used to reduce dose in abdominal CT such as modifying scanning parameters [29], using a combination of standarddose and low-dose phases in a CTU protocol [30] and reducing the number of acquisitions and scan lengths [14]. As the implementation of the LDCT has been very slow, this study provides an insight into the possible applicability of this protocol in clinical practice to reduce radiation dose for adolescents and children, as well as patients that require repetitive imaging.
Our CTU protocol is optimized using automatic dose modulation as well as iterative reconstruction and combination of low-dose and standard-dose series in concurrence with Dahlman et al. [30] who obtained significant dose reductions, in the unenhanced and excretory phases, achieved when combined with one normal-dose phase. However, the dilemma of reducing the radiation burden in certain patient groups still remains. Some institutions have adopted a workflow routine in order to minimize the number of phases required by viewing the low-dose series to determine further need for imaging (Magnusson A, CT lecture, Larvik, 2018, personal communications). However due to logistics this is not always possible especially for referrals from the emergency department where time is of essence.

One of the sites at our institution extensively adopted the LDCT in 2012, to meet the urologists needs for imaging after Extracorporal Shockwave Lithotripsy (ESWL) as well as follow-up imaging of patients with nephro- and uro-lithiasis. With growing expertise and increasing diagnostic confidence, the comfort zone boundaries were broadened and the LDCT use was extended to diagnostics of acute flank pain and acute abdomen for several indications such as diverticulitis, appendicitis and renal calculi [22] in concurrence with Hamimi et al. [31] who studied the efficiency of low-dose technique using an effective mAs of 50 (very similar to our LDCT protocol), in diagnosis of renal calculi and concluded that it was crucial in the management of renal stone disease in the acute setting. Both Lee et al. [12] and Poletti et al. [11] demonstrated the use of LDCT in acute abdominal pain diagnoses. An LDCT protocol can be considered as a valuable tool in acute abdominal pain evaluation as it allows for many possible differential diagnoses, but radiologists should also be aware of its limitations [22].

The statistical method (VGR) that we used, allowed us to analyze the three phases in the same analysis, with subsequent pair-wise comparisons. VGR also has the ability to let the researcher estimate the potential dose reduction resulting from changes in the imaging protocol. However, the design of this retrospective study does not permit such an analysis.

There is one major study limitation: due to the retrospective nature of our study we failed to separate the individual effects of dose and contrast enhancement on image quality. Ideally to determine these effects individually, all three phases should have been compared at different dose levels which would also allow for estimation of the potential dose reduction for each individual phase [32]. However, this would have increased the radiation burden for these patients.

\section{Conclusion}

Visualisation of renal anatomy in the three phases were as expected with each post-contrast phase showing favourable scores compared to the native phase. No 


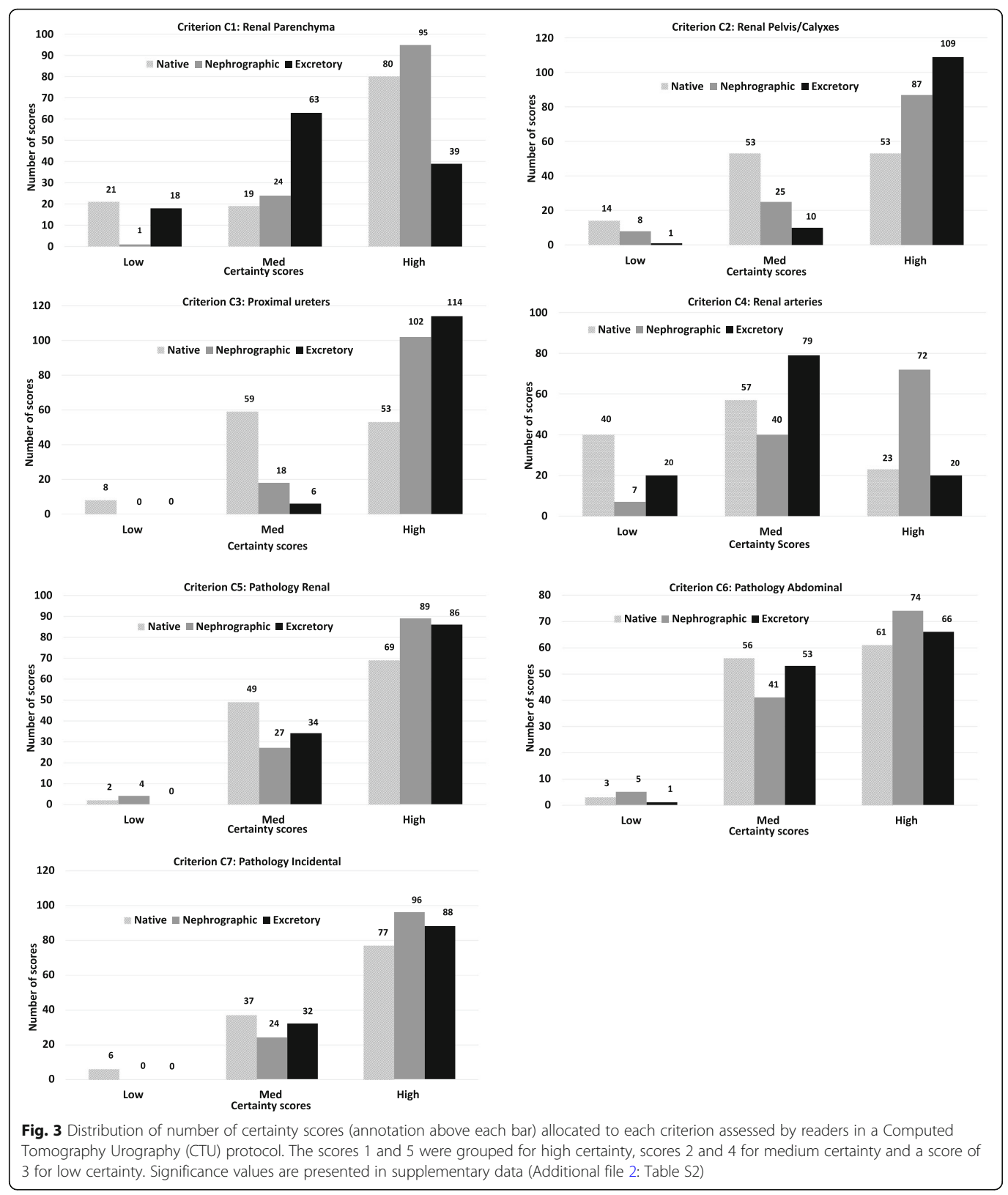

statistically significant differences in the assessment of pathology were found between the three phases.

Since many certainty scores were in the high and medium categories, the LDCT seems to be sufficient to differentiate between normal and pathological examinations. In order to reduce the radiation burden in adolescents and children, as well as patients with negative outcomes and those that require repetitive imaging, the LDCT could be considered a suitable alternative as a first line imaging method. However, radiologists should be aware of its limitations. 


\section{Additional files}

Additional file 1: Table S1. Scores for each of the criteria. Significance of differences between phases tested with mixed-effects ordinal logistic regression, with pairwise comparisons using Bonferroni correction. (DOCX 63 kb)

Additional file 2: Table S2. Certainty scores for each of the criteria. Significance of differences between phases tested with mixed-effects ordinal logistic regression in phase comparisons using Bonferroni correction. (DOCX $55 \mathrm{~kb}$ )

\section{Abbreviations}

AHARA: As high as reasonably achievable; ALARA: As low as reasonably achievable; $\mathrm{CTDI}_{\text {vol }}$ : Computed Tomography Dose Index; CTU: Computed Tomography Urography; DLP: Dose Length Product; ESWL: Extracorporal Shock Wave Lithotripsy; kV: kilovolt; LDCT: Low-dose Computed Tomography; mAs: milliampere seconds; mGy: milligray; MRI: Magnetic Resonance Imaging; mSv: millisievert; SCP: Standardized Care Pathway; SD: Standard deviation; SSDE: Size Specific Dose Estimate

\section{Acknowledgements}

We thank our participating radiologists, Anki Pozson, Jenny Öman and Senija Halilic for grading the images.

\section{Authors' contributions}

All authors (BK, JNA, ÖS, AP, HS and MS) have contributed to the conception, design of the work and interpretation of the data. BK has been responsible for acquisition, drafting and revision of the manuscript in collaboration with the other authors and ÖS for performing the statistical analysis. All authors have contributed to the final manuscript and approved it.

\section{Funding}

This work was supported by ALF- and LFoU-grants from Region Östergötland and the Medical Faculty at Linköping University. The funding bodies have had no role in the design of the study and collection, analysis, interpretation of data and in writing the manuscript.

\section{Availability of data and materials}

The datasets used and/ or analyzed during the current study are available from the corresponding author on reasonable request.

\section{Ethics approval and consent to participate}

All procedures performed in studies involving human participants were in accordance with the ethical standards of the institutional research committee (Linköping University) and with the 1964 Helsinki declaration and its later amendments or comparable ethical standards. Our regional Ethical Review Board (EPN, Linköping) approved this retrospective study, and the requirement for informed consent was waived.

\section{Consent for publication}

Not applicable.

\section{Competing interests}

The authors declare that they have no competing interests.

\section{Author details}

'Department of Radiology, Linköping University, Linköping, Sweden. ${ }^{2}$ Department of Medical \& Health Sciences, Linköping University, Linköping, Sweden. ${ }^{3}$ Center for Medical Image Science \& Visualization (CMIV), Linköping University, Linköping, Sweden. ${ }^{4}$ Department of Medical Physics, Linköping University, Linköping, Sweden. ${ }^{5}$ Department of Biomedical Engineering and Health Systems (MTH), KTH Royal Institute of Technology, Stockholm, Sweden.

Received: 16 April 2019 Accepted: 29 July 2019

Published online: 09 August 2019

\section{References}

1. Sudah M. Modern Imaging of the Upper Urinary Tract [Dissertations in Health Sciences]: Publications of the University of Eastern Finland. Kuopio:
University of Eastern Finland; 2016. http://epublications.uef.fi/pub/urn_isbn_ 978-952-61-2083-6/urn_isbn_978-952-61-2083-6.pdf.

2. IAEA Safety standards - Radiation protection and safety of radiation sources International Basic Safety standards. Vienna: International Atomic Energy Agency; 2014. https:/www-pub.iaea.org/MTCD/Publications/PDF/Pub1578_ web-57265295.pdf.

3. Kalra M, Sodickson AD, Mayo-Smith WW. CT radiation key concepts for gentle and wise use. Radiographics. 2015;35:1706-21.

4. Cowan NC. CT urography for hematuria. Nat Rev Urol. 2012;9(4):218-26.

5. Bhatt $\mathrm{K}$, Monga M, Remer EM. Low-dose computed tomography in the evaluation of urolithiasis. J Endourol. 2015:29(5):504-11.

6. Standardiserade vårdförlopp cancer-urinblasa-ovre-urinvagarna www. cancercentrum.se Regionala cancercentrum; 2018 [updated 27th February 2018]. https://www.cancercentrum.se/globalassets/cancerdiagnoser/ urinvagar/urinblase\%2D\%2Doch-urinrorscancer/vardforlopp/svf-cancerurinblasa-ovre-urinvagarna.pdf. Accessed 15 Dec 2018.

7. Muthulakshmi M, Gopalakrishnan S. Study on urinary tract infection among females of reproductive age group in a rural area of Kancheepuram district, Tamil Nadu. Int J Community Med Public Health. 2017;4(10):3915-21.

8. Van Der Molen AJ, Cowan NC, Mueller-Lisse UG, Nolte-Ernsting CC, Takahashi S, Cohan RH. CT urography: definition, indications and techniques. A guideline for clinical practice. Eur Radiol. 2008;18:4-17.

9. Mathews JD, Forsythe AV, Brady Z, Butler MW, Goergen SK, Byrnes GB, et al Cancer risk in 680,000 people exposed to computed tomography scans in childhood or adolescence: data linkage study of 11 million Australians. BMJ. 2013;346:f2360.

10. Kaza RK, Platt JF, Goodsitt MM, Al-Hawary MM, Maturen KE, Wasnik AP, et al. Emerging techniques for dose optimization in abdominal CT. Radiographics. 2014;34:4-17.

11. Poletti PA, Becker M, Becker CD, Halfon Poletti A, Rutschmann OT, Zaidi $H$, et al. Emergency assessment of patients with acute abdominal pain using low-dose CT with iterative reconstruction: a comparative study. Eur Radiol. 2017;27:3300-9.

12. Lee KH, Shim YS, Park SH, Choi SJ, Pak SY, Cheong H. Comparison of standard-dose and half-dose dual-source abdominopelvic $C T$ scans for evaluation of acute abdominal pain. Acta Radiol. 2018;0:1-9.

13. Safety and efficacy of computed tomography (SECT): A broad perspective (CT Safety and Efficacy) Euratom call 2003. 2008 http://www.biophysicssite. com/Documents/SECT2008/DeliverableWP1D5F.pdf. Accessed 5 Feb 2019.

14. Sung MK, Singh S, Kalra MK. Current status of low dose multi-detector $C T$ in the urinary tract. World J Radiol. 2011;3:256-65.

15. Rodger F, Roditi G, Aboumarzouk OM. Diagnostic accuracy of low and ultralow dose $C T$ for identification of urinary tract stones: a systematic review. Urol Int. 2018;100:375-85.

16. Kim K, Kim YH, Kim SY, Kim S, Lee YG, Kim KP, et al. LDCT for evaluating appendicitis. N Engl J Med. 2012;366:1596-605.

17. Bonn-call-for-action 10 actions to improve Radiation Protection. Vienna: IAEA; 2014. https://www.who.int/ionizing_radiation/medical_exposure/ bonncallforaction2014.pdf?ua=1. Accessed 15 Dec 2018.

18. IAEA Bulletin 52-2-2011 Division of Public Information: IAEA; 201 https://www.iaea.org/sites/default/files/bull52-2-february2011.pdf. Accessed 10 Feb 2019.

19. Dose reduction in $C T$ while maintaining diagnostic confidence: a feasibility/ demonstration study. Vienna: IAEA; 2009. ISNN 1011-4289 https://www-pub. iaea.org/MTCD/Publications/PDF/te_1621_web.pdf. Accessed 7 Feb 2019.

20. Gervaise A, Gervaise-Henry C, Pernin M, Naulet P, Junca-Laplace C, LapierreCombes M. How to perform low-dose computed tomography for renal colic in clinical practice. Diagn Interv Imaging. 2016;97:393-400

21. Weisenthal K, Karthik P, Shaw M, Sengupta D, Bhargavan-Chatfield M, Burleson J, et al. Evaluation of kidney stones with reduced-radiation dose CT: Progress from 2011-2012 to 2015-2016-not there yet. Radiology. 2018; 286:581-9.

22. Halilic S and Kämmerling N. Värdet av DT-buköversikt som primär undersökning vid akut buk smärta. Linköping University Electronic Press, Linköping, 2016. http://liu.diva-portal.org/smash/get/diva2:1304503/ FULLTEXT01.pdf. Accessed 12 Apr 2019.

23. Wang $\mathrm{J}-\mathrm{H}$, Shen $\mathrm{S}-\mathrm{H}$, Huang $\mathrm{S}-\mathrm{S}$, Chang $\mathrm{C}-\mathrm{Y}$. Prospective comparison of unenhanced spiral computed tomography and intravenous urography in the evaluation of acute renal colic. J Chin Med Assoc. 2008;71(1):30-6.

24. Alshamari M, Norrman E, Geijer M, Jansson K, Geijer H. Diagnostic accuracy of low-dose $C T$ compared with abdominal radiography in non-traumatic 
acute abdominal pain: prospective study and systematic review. Eur Radiol. 2016;26(6):1766-74

25. The report of AAPM task group 204. Size-specific dose estimates in paediatric and adult body CT examinations, 2011. https://www.aapm.org/ pubs/reports/RPT_204.pdf. Accessed 6 June 2019.

26. Boos J, Kropil P, Bethge OT, Aissa J, Schleich C, Sawicki LM, et al. Accuracy of size-specific dose estimate calculation from center slice in computed tomography. Radiat Prot Dosim. 2018;178(1):8-19.

27. Smedby Ö, Fredrikson M. Visual grading regression: analysing data from visual grading experiments with regression models. Br J Radiol. 2010; 83(993):767-75

28. Saffari SELA, Fredrikson M, Smedby O. Regression models for analyzing radiological visual grading studies--an empirical comparison. BMC Med Imaging. 2015;15:49):1-10.

29. Dyakov I, Alamin M, Groudeva V, Vassileva J, Stoinova V, Hadjidekov V. Optimisation of $C T$ procedures in two radiology departments. Phys Med. 2014;30:e17.

30. Dahlman P, Van der Molen AJ, Magnusson M, Magnusson A. How much dose can be saved in three-phase $\subset T$ urography? A combination of normaldose corticomedullary phase with low-dose unenhanced and excretory phases. AJR Am J Roentgenol. 2012;199:852-60.

31. Hamimi A, El Azab M. MSCT renal stone protocol; dose penalty and influence on management decision of patients: is it really worth the radiation dose? Egypt J Radiol Nucl Med. 2016:47:319-24.

32. Kataria B, Althen JN, Smedby O, Persson A, Sokjer H, Sandborg M Assessment of image quality in abdominal $C$ : potential dose reduction with model-based iterative reconstruction. Eur Radiol. 2018;28(6):2464-73.

\section{Publisher's Note}

Springer Nature remains neutral with regard to jurisdictional claims in published maps and institutional affiliations.

\section{Ready to submit your research? Choose BMC and benefit from:}

- fast, convenient online submission

- thorough peer review by experienced researchers in your field

- rapid publication on acceptance

- support for research data, including large and complex data types

- gold Open Access which fosters wider collaboration and increased citations

- maximum visibility for your research: over $100 \mathrm{M}$ website views per year

At $\mathrm{BMC}$, research is always in progress.

Learn more biomedcentral/com/submissions 



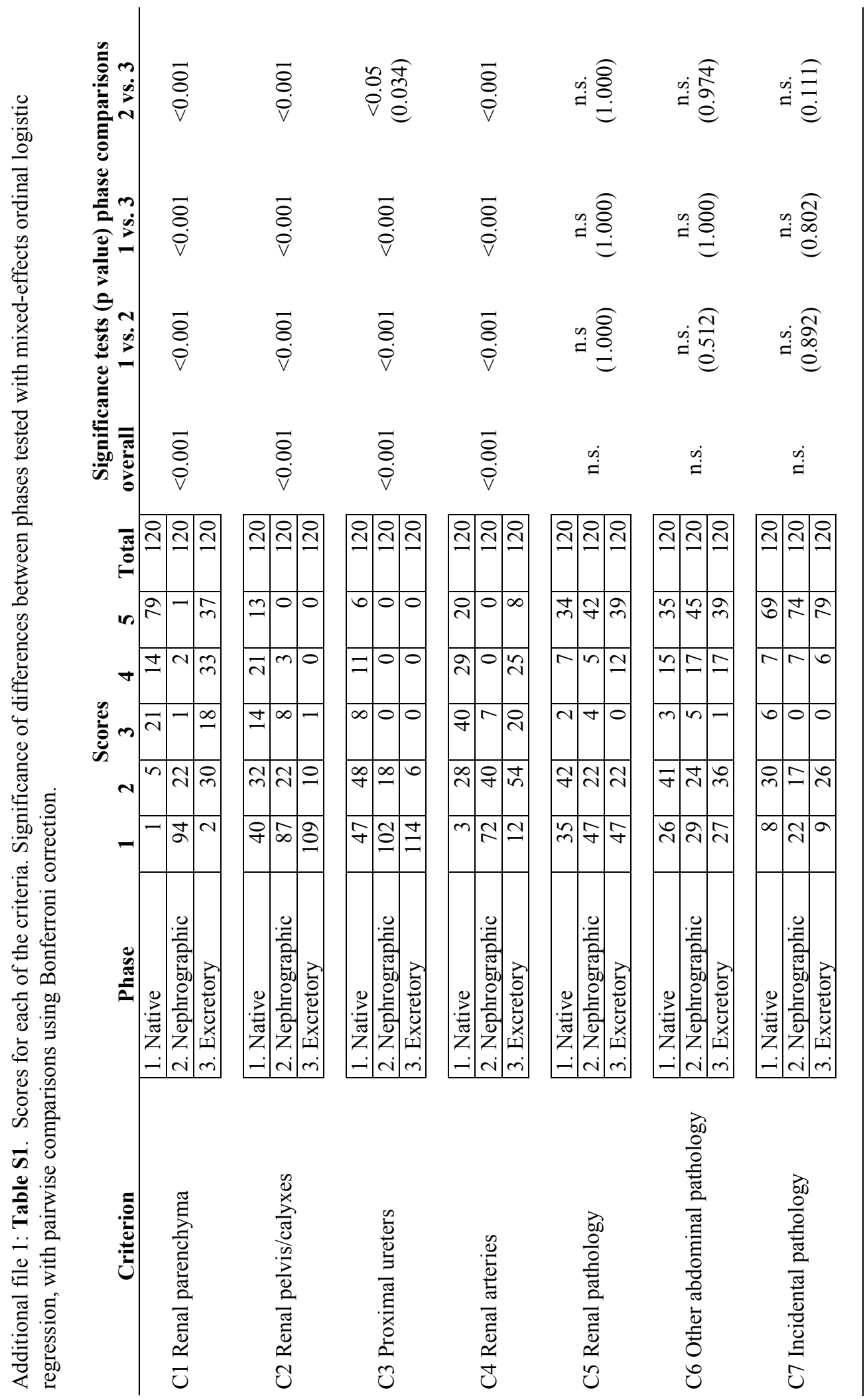




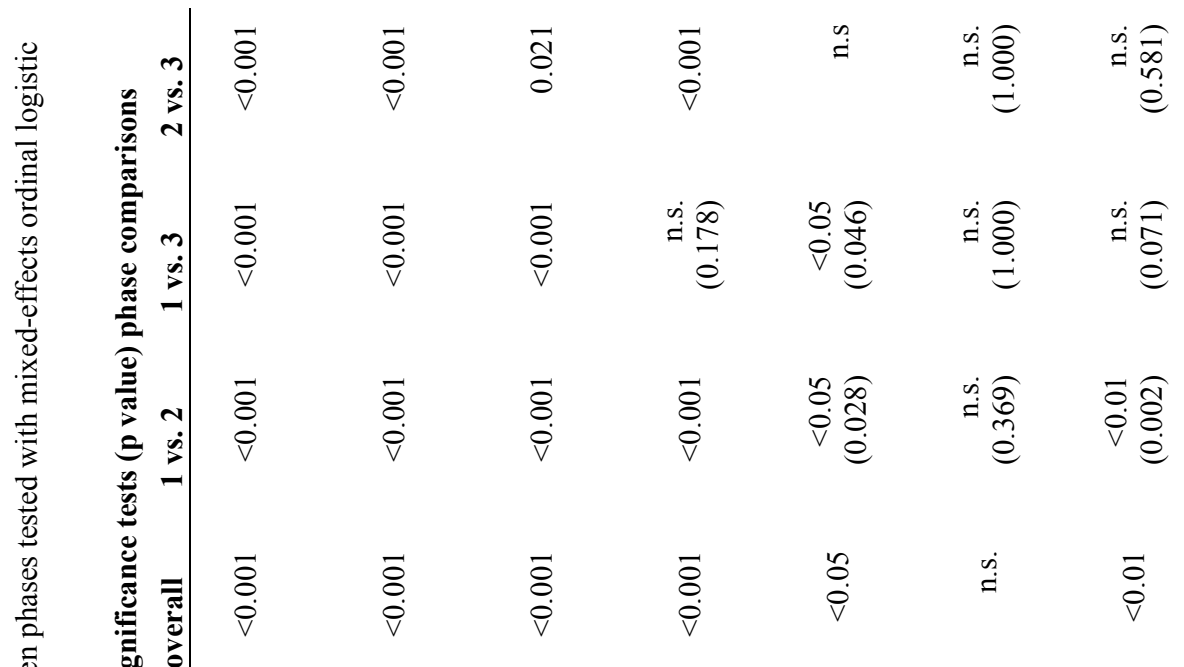

这

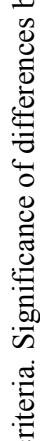

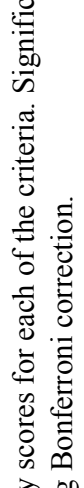

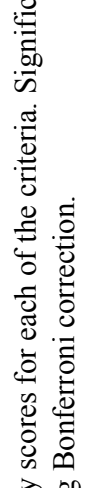

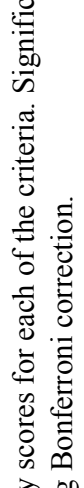

홍 ปิ

ิㅗ옹

ิㅣㅇㅝ

ิㅣㅇㅝ

이오옳

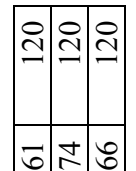

으

ธี

กำ

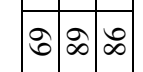

ก

4ั $\quad 0$

离

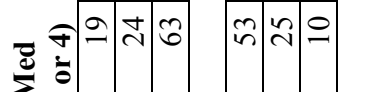
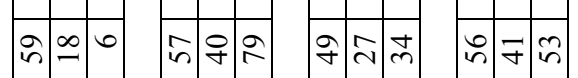

กิัก
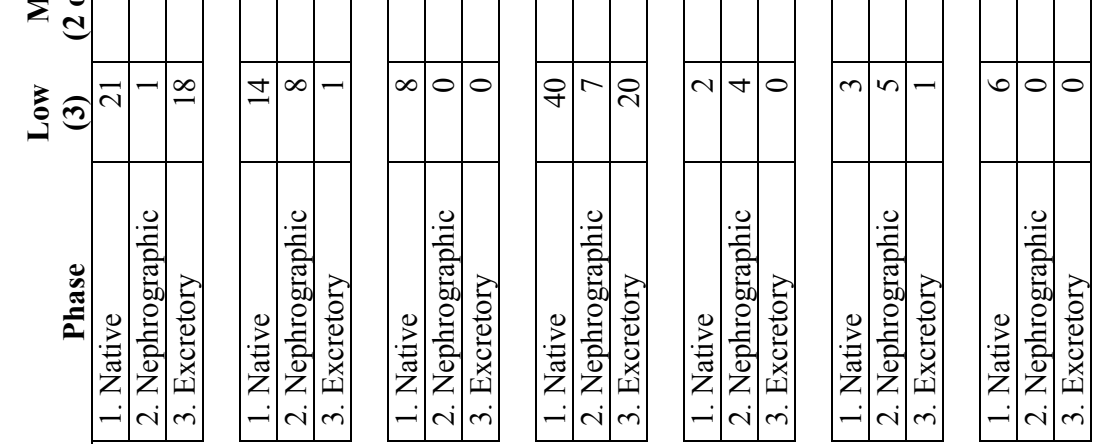

ยั

นี

ก है

苛

运

우

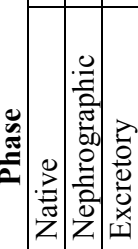

$-\dot{i} \dot{r}$
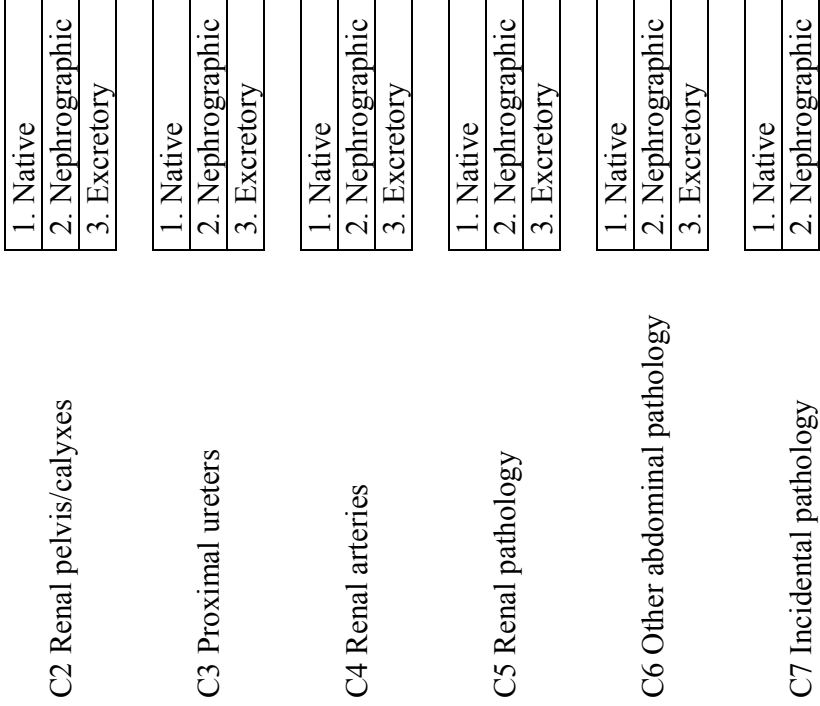
PAPER IV 

Title: Assessment of image quality in abdominal computed tomography: Effect of model-based iterative reconstruction, multi-planar reconstruction and slice thickness on potential dose reduction.

Supervisors:

Prof. Michael Sandborg

Prof. Örjan Smedby

Prof. Anders Persson

Ass. Prof. Hannibal Sökjer

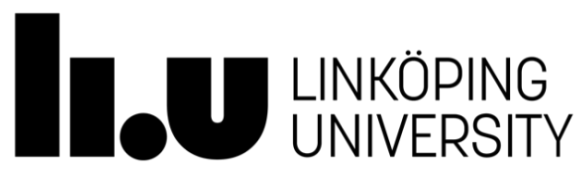

Department of Medical and Health Sciences

Linköping University, Sweden

Linköping 2019 


\section{Title: Assessment of image quality in abdominal computed}

tomography: Effect of model-based iterative reconstruction, multiplanar reconstruction and slice thickness on potential dose reduction. Authors:

Bharti Kataria BSc, MSc (Corresponding Author)

Department of Radiology, Department of Medical \& Health Sciences, Center for Medical Image Science \& Visualization (CMIV), Linköping University, Linköping, Sweden

E-mail: Bharti.Kataria@liu.se telephone: +46103 32775 Fax: +46103 32709

Address: County Council of Östergötland, DC

Department of Radiology,

S-581 85 Linköping,

Sweden

Jonas Nilsson Althén, MSc

Department of Medical Physics, Department of Medical \& Health Sciences, Linköping

University, Linköping, Sweden

E-mail: Jonas.Althen.Nilsson@ regionostergotland.se

Address: County Council of Östergötland, DC

Department of Medical Physics

S-581 85 Linköping,

Sweden

Örjan Smedby MD, PhD

Department of Biomedical Engineering and Health Systems (MTH), KTH Royal Institute of Technology, Stockholm, Sweden.

E-mail: Orjan.Smedby@sth.kth.se

Address: KTH Royal Institute of Technology

Department of Biomechanical Engineering and Health Systems

SE-141 52 Huddinge, Stockholm

Sweden

Anders Persson MD, PhD

Department of Radiology, Department of Medical \& Health Sciences, Center for Medical Image Science \& Visualization (CMIV), Linköping University, Linköping, Sweden

E-mail: Anders.Persson@cmiv.liu.se

Address: Center for Medical Image Science \& Visualization

Department of Radiology,

County Council of Östergötland, DC

S-581 85 Linköping,

Sweden 
Hannibal Sökjer MD, PhD

Department of Medical \& Health Sciences, Linköping University, Linköping, Sweden

E-mail: Hannibal.Sokjer@gmail.com

Address: Department of Medical \& Health Sciences

Linköping University,

S-581 83 Linköping,

Sweden

\section{Michael Sandborg PhD}

Department of Medical Physics, Department of Medical \& Health Sciences, Center for Medical Image Science \& Visualization (CMIV), Linköping University, Linköping, Sweden

E-mail: Michael.Sandborg@liu.se

Address: County Council of Östergötland, DC

Department of Medical Physics

S-581 85 Linköping,

Sweden 


\begin{abstract}
Purpose: To determine the effect of tube load, model-based iterative reconstruction (MBIR) strength and slice thickness in abdominal CT using visual comparison of multi-planar reconstruction images.
\end{abstract}

Method: Five image criteria were assessed independently by four radiologists on two data sets at 42- and 98-mAs tube loads for 25 patients examined on a 192-slice dual-source CT scanner. The effect of tube load, MBIR strength and potential dose reduction was estimated with Visual Grading Regression (VGR). Objective image quality was determined by measuring noise (SD, contrast-to-noise (CNR) ratio and noise-power spectra (NPS).

Results: Comparing 42- and 98-mAs tube loads, improved image quality was observed as a strong effect of log tube load regardless of MBIR strength $(p<0.001)$. Comparing strength 5 to 3 , better image quality was obtained for two criteria $(p<0.01)$, but inferior for liver parenchyma and overall image quality. Image quality was significantly better for slice thicknesses of $2 \mathrm{~mm}$ and $3 \mathrm{~mm}$ compared to $1 \mathrm{~mm}$, with potential dose reductions between $24 \%$ to $41 \%$. As expected, with a decrease in slice thickness and algorithm strength, the noise power and SD (HU-values) increased, while the CNR decreased.

Conclusion: Increasing slice thickness from $1 \mathrm{~mm}$ to $2 \mathrm{~mm}$ or $3 \mathrm{~mm}$ allows for a possible dose reduction. MBIR strength 5 shows improved image quality for three out of five criteria for $1 \mathrm{~mm}$ slice thickness. Increasing MBIR strength from 3 to 5 has diverse effects on image quality. Our findings do not support a general recommendation to replace MBIR strength 3 by strength 5 in clinical abdominal CT protocols.

Keywords: Computed Tomography; Abdomen; Iterative reconstruction; Dose, Slice thickness; multi-planar reconstruction (MPR) 
Abbreviations

ADMIRE Advanced Modeled Iterative Reconstruction

AD3 ADMIRE strength 3

AD5 ADMIRE strength 5

ALARA As low as reasonably achievable

BMI Body mass index

CNR Contrast-to-noise ratio

CTDI $_{\text {vol }} \quad$ Volume CT Dose Index

DLP Dose-Length-Product

DR Dose reduction

FBP Filtered back projection

HU Hounsfield Units

IR Iterative reconstruction

MBIR Model-based iterative reconstruction

MPR Multi-planar reconstruction

NPS Noise power spectrum

Qref Quality reference

ROI Region of interest

SSDE Size-specific dose estimate

VGR Visual Grading Regression 


\section{Introduction}

Although modern CT equipment and technique development have contributed to overall lower radiation doses, the collective population dose is still increasing [1]. Standardization and optimization of clinical protocols is therefore advocated to keep the radiation dose as low as reasonably achievable (ALARA) [2] while maintaining diagnostic confidence. This can be achieved by using technological advancements such as dose modulation and iterative reconstruction (IR) methods [3, 4].

The diagnostic performance and effect of model-based iterative reconstruction (MBIR) on different aspects of image quality and potential dose reductions has been studied by several peers [5-9]. However, due to an increase in image noise, dose reduction impairs the diagnostic confidence as depiction of low-contrast lesions in abdominal imaging is compromised. To maintain the high spatial resolution and minimize partial volume averaging effects, CT images are acquired with optimized radiation dose and the thinnest detector configuration available [10]. Various MBIR studies have evaluated the effect of variation in slice thickness on image quality $[7,11,12]$. In addition, MPR provides improved visualization of abdominal structures and increases the diagnostic confidence [13].

Previous visual evaluation performed on advanced modelled iterative reconstruction (ADMIRE) strength 3 (AD3) and 5 (AD5) showed a preference for AD3 over AD5 [5]. To determine the desired clinical image quality, an evaluation of both subjective and objective image quality parameters is a prerequisite when a new image reconstruction technique is introduced into the clinical routine [9]. The performance of thin slices of ADMIRE and comparison of simultaneous MPR images in 3-planes (corresponding to the clinical set up), in clinical non-enhanced abdominal CT, have to the best of our knowledge, not been studied before. 
Our aim was to evaluate the image quality in MPR images with both a subjective and an objective approach in non-enhanced abdominal CT, and to explore the effect of ADMIRE strength and slice thickness on possible dose reduction.

\section{Material \& Methods}

This is a prospective study approved by the regional ethical board. Written informed consent was obtained from all study patients before the CT examination. Three data sets at reference tube loads of $42 \mathrm{mAs}(30 \%), 98 \mathrm{mAs}(70 \%)$ and $140 \mathrm{mAs}(100 \%)$ were acquired using a 192slice dual source CT scanner (Siemens Healthineers) in the dual source mode by splitting the tube load proportionately between the two sources.

Images acquired at tube loads $42 \mathrm{mAs}$ and $98 \mathrm{mAs}$ are included in the present assessment as previous evaluation indicated no significant improvement in image quality between $98 \mathrm{mAs}$ and $140 \mathrm{mAs}$ [5]. Twenty-five patients who underwent a clinically indicated non-enhanced abdominal CT were included, and demographic data (age, height, mass) were recorded. The CT dose descriptors, Volume CT dose index $\left(\mathrm{CTDI}_{\mathrm{vol}}\right)$ and dose-length-product (DLP) were retrieved and size-specific dose estimate (SSDE) computed based on the antero-posterior and lateral patient dimensions[14]. Acquisition parameters are presented in Table 1.

\section{Visual assessment}

Images obtained at $42 \mathrm{mAs}$ and $98 \mathrm{mAs}$ using iterative algorithms AD3 and AD5 were reconstructed with MPR in the axial, coronal and sagittal planes, and slice thicknesses at $3 \mathrm{~mm}$, $2 \mathrm{~mm}$ and $1 \mathrm{~mm}$ with increment of $2 \mathrm{~mm}, 1 \mathrm{~mm}$ and $0.5 \mathrm{~mm}$ respectively. The MPR images were graded by four radiologists with varying experience ( 8 -24 years) using 4 criteria (C1-C4) from the European guidelines for image quality in abdominal CT [15] together with overall image quality (C5) to suit the purpose of this study (Table 2). 
Table 1. Acquisition parameters for 192-slice dual source scanner in dual source mode with split mAs between the two sources to produce two stacks of images at $42 \mathrm{mAs}$ and $98 \mathrm{mAs}$.

\begin{tabular}{ccccccccc}
\hline & Fixed & Qref & & & & Care & & Dose \\
Source & $\mathrm{kV}$ & $\mathrm{mAs}$ & Acquisition & Rotation & Pitch & Dose 4D & Kernel & level \\
\hline Tube A & 120 & 98 & $192 \times 0.6$ & $0.5 \mathrm{~s}$ & 0.6 & Yes & $\mathrm{Br} 36$ & $70 \%$ \\
Tube B & 120 & 42 & $192 \times 0.6$ & $0.5 \mathrm{~s}$ & 0.6 & Yes & $\mathrm{Br} 36$ & $30 \%$ \\
*Full dose & 120 & 140 & $192 \times 0.6$ & $0.5 \mathrm{~s}$ & 0.6 & Yes & Br36 & $100 \%$ \\
\hline
\end{tabular}

Qref = Quality reference $m A s$

*Full dose images obtained by combining data from Tubes $A+B$. Clinical standard multi-planar reconstruction in 3 planes with slice thickness at $3 \mathrm{~mm}$ with an increment of $2 \mathrm{~mm}$.

To achieve a similar understanding of the evaluation of the image criteria, a coaching session was held for the participating radiologists. These data sets are not included in the study. The images were displayed in random order on PACS workstations with simultaneous pairwise 3plane MPR comparisons in the same patient randomly assigned to right or left monitor (Figure 1). Each observer independently graded the image stacks using a 5-point Likert-type scale (Table 2).

Table 2. Image quality criteria assessed in pairwise comparison of images reconstructed with 3-plane multiplanar reconstruction (MPR) and graded on a 5-point Likert-type scale.

\section{Visual Grading image quality criteria}

C1: Visually sharp reproduction of the liver parenchyma

C2: Visually sharp reproduction of the pancreas contour

C3: Visually sharp reproduction of the contours of the kidneys \& proximal ureters

C4: Reproduction of contours of lymph nodes $<15 \mathrm{~mm}$ in diameter

C5: Overall image quality for diagnostic purposes

\section{Grading scores}

-2 Images on left monitor are better than images on right monitor

-1 Images on left monitor are probably better than images on right monitor

0 Images on left and right monitor are equivalent

+1 Images on right monitor are probably better than images on left monitor

+2 Images on right monitor are better than images on left monitor 


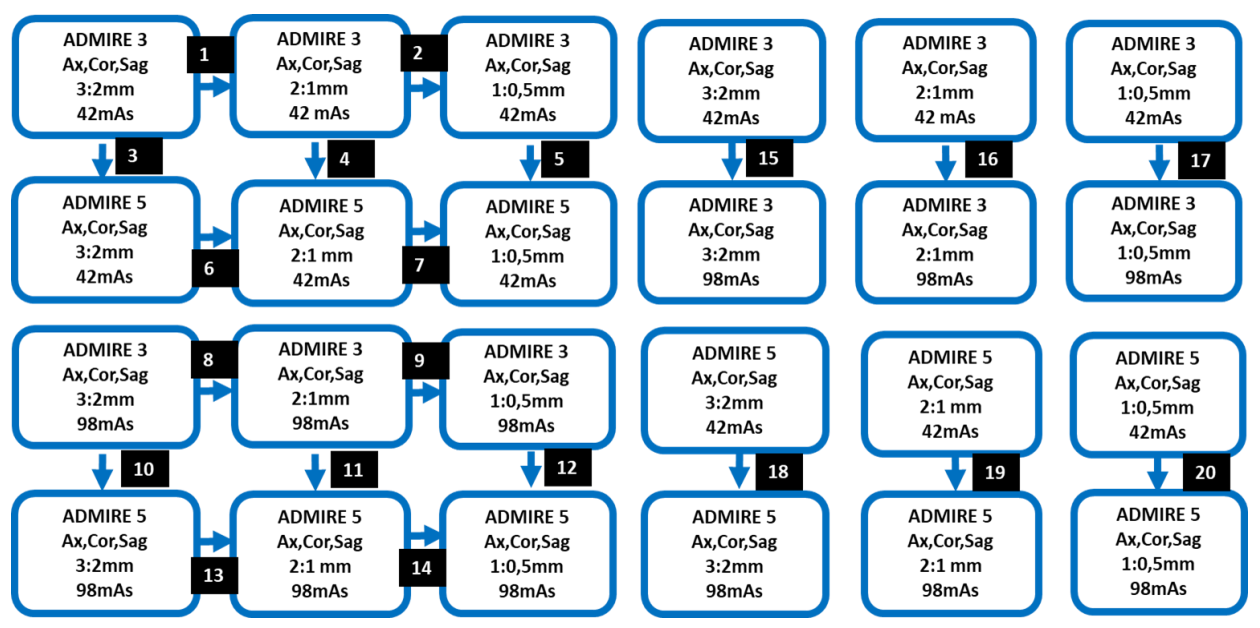

Figure. 1 Schematic diagram of MPR in 3-planes acquired at tube loads $42 \mathrm{mAs}$ and $98 \mathrm{mAs}$, reconstructed using ADMIRE strengths 3 and 5 with arrows and numbers showing the pairwise comparisons performed.

\section{Quantitative assessment}

Quantitative measurements were performed in phantoms imaged with the same acquisition parameters as the patients. Standard deviation (SD) in Hounsfield Units (HU), contrast-to-noise ratio (CNR), and noise-power spectra (NPS) were measured in images of an anthropomorphic abdominal phantom with an extension ring (QRM, GmbH).

The SD was measured in $32^{2}$ ROI pixels in the liver. The CNR was derived as the absolute difference in $\mathrm{HU}$-values in $32^{2}$ region of interest (ROI) pixels in the liver region and the adjacent background material divided by the SD of the HU-values in the background material using the formula:

$$
C N R=\left|H U_{\text {liver }}-H U_{\text {background }}\right| / S D_{\text {background }}
$$

The 2D NPS was computed using the expression from Verdun et al. [16], with pixel sizes 0.85 $\mathrm{mm}$ and $64^{2}$ ROI pixels in the liver region. From the average 2D NPS over adjacent slices, the radial 1-dimensional NPS was computed as function of spatial frequency, $f=\sqrt{f_{x}{ }^{2}+f_{y}{ }^{2}}$ $(1 / \mathrm{mm})$ where, $f_{\mathrm{x}}$ and $f_{\mathrm{y}}$ are the spatial frequencies in the $\mathrm{x}$ and $\mathrm{y}$ directions in the 2D NPS. 


\section{Statistical analysis}

Visual Grading Regression (VGR) [17] was used to calculate potential dose reduction depending on tube load, reconstruction algorithm and slice thickness using the multi-level mixed-effects ordered logistic (meologit) command in Stata 13.1 (Stata Corporation LP) software. Variation in image quality due to choice of tube load, reconstruction algorithm and slice thickness is described by the regression coefficients, and estimation of potential dose reduction $(D R)$ is acquired by relating two of these coefficients to each other [18] when replacing one algorithm with another from the equation $D R=1-e^{-(b / a)}$, where $a$ is the regression coefficient for $\log \mathrm{mAs}$ and $b$ for the iterative reconstruction algorithm or slice thickness. The null hypothesis was that neither tube load, reconstruction strength nor slice thickness influence perceived image quality.

Interobserver reliability was described with the weighted kappa, calculated using the kappa2 command in Stata. The significance limit was set at $p=0.05$.

\section{Results}

The patient population consisted of 13 females and 12 males, age range 53-92 years (mean 71.6, SD 10.1) with a Body Mass Index (BMI) $\left(\mathrm{kg} / \mathrm{m}^{2}\right)$ ranging from 17.3-26.2 (mean 22.8, SD 2.14). The $\mathrm{CTDI}_{\mathrm{vol}}$ (mGy) ranged from 4.4-8.3 (mean 6.4, SD 1.2), DLP (mGy $\mathrm{cm}$ ) ranged from 194 -385 (mean 303, SD 61.8) and SSDE (mGy) ranged from 6.2-12.2 (mean 8.7, SD 1.3).

\section{Visual assessment}

Visual image quality in MPR images of a 65-year-old patient who underwent a clinically indicated non-enhanced abdominal CT are presented in Figure 2. 


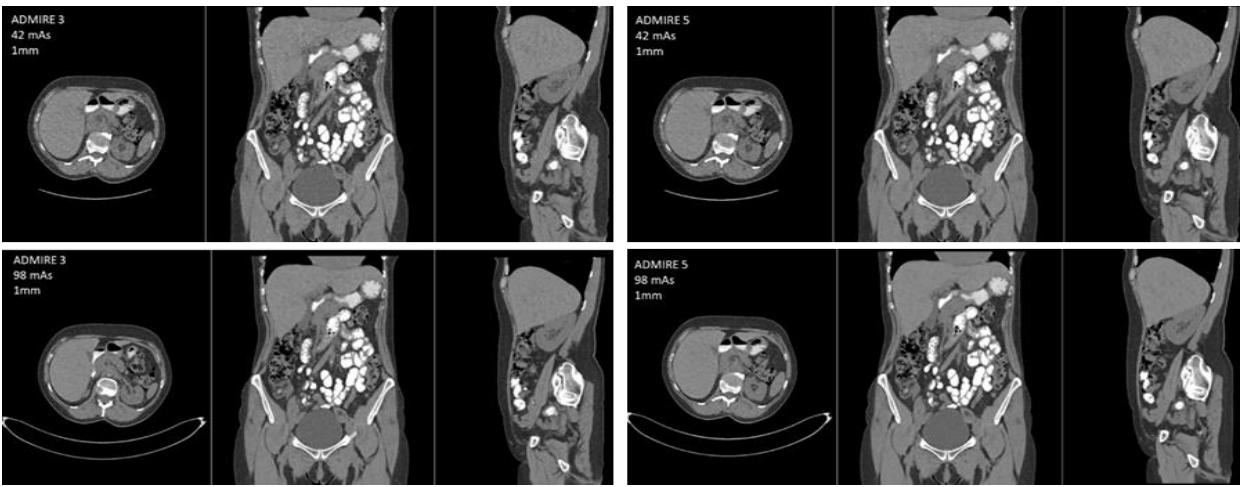

A. Multi-planar images reconstructed with $1 \mathrm{~mm}$ slice thickness
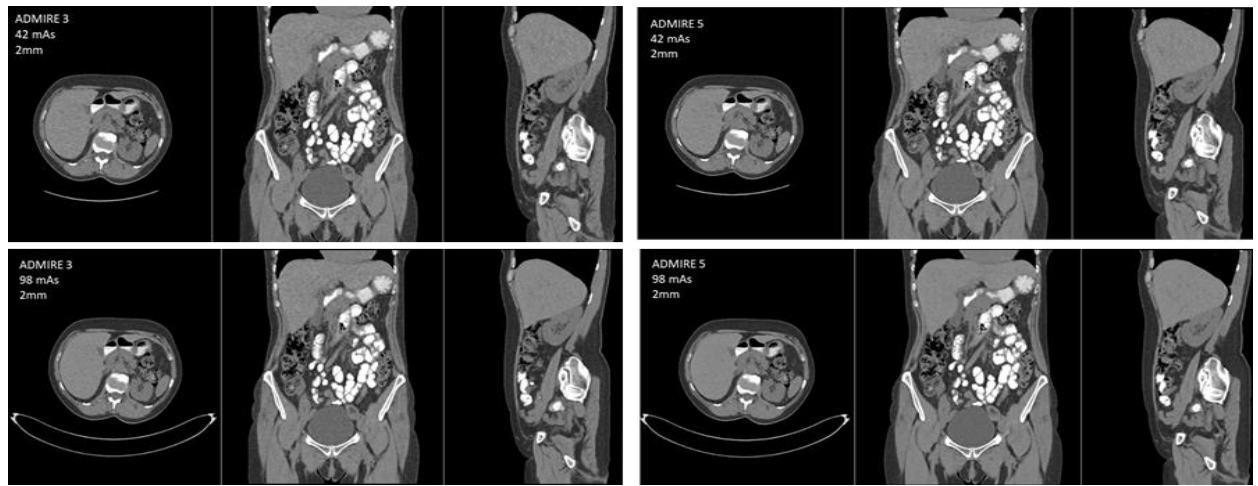

B. Multi-planar images reconstructed with $2 \mathrm{~mm}$ slice thickness
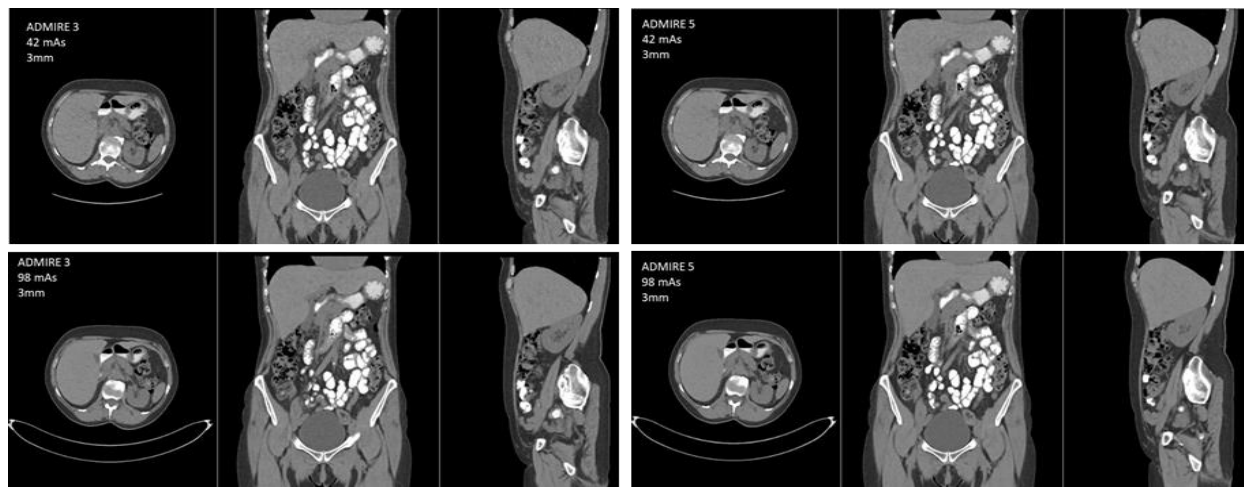

C. Multi-planar images reconstructed with $3 \mathrm{~mm}$ slice thickness

Figure 2 Multi-planar 3-plane images showing the visual image quality in a non-enhanced abdominal CT reconstructed with ADMIRE strengths 3 and 5, at tube loads $42 \mathrm{mAs}$ and $98 \mathrm{mAs}$ and 3 slice thicknesses. 


\section{Effect of tube load}

Comparing $42 \mathrm{mAs}$ and $98 \mathrm{mAs}$ tube loads in all available data, significantly improved image quality $(p<0.001)$ was observed for the higher tube load, regardless of ADMIRE strength for all criteria (Figure 3, Table 3). Similar results were observed when analyzing only one slice thickness individually (Table 4A-C).

a)

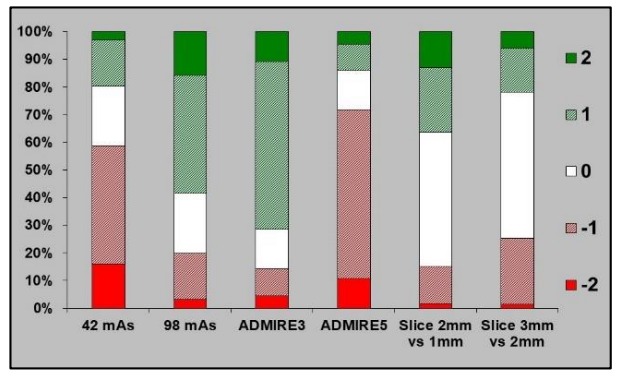

c)

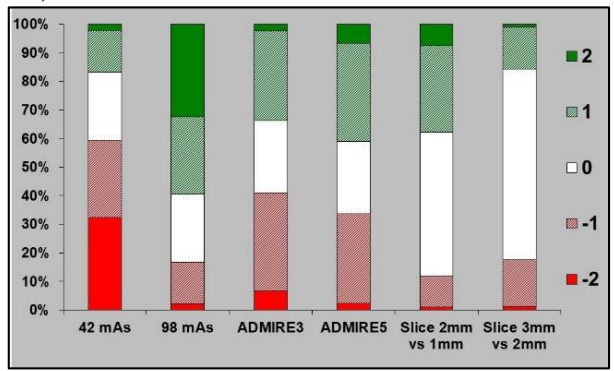

e) b)

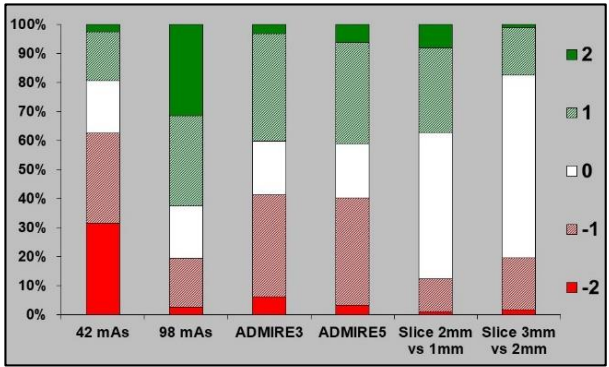

d)

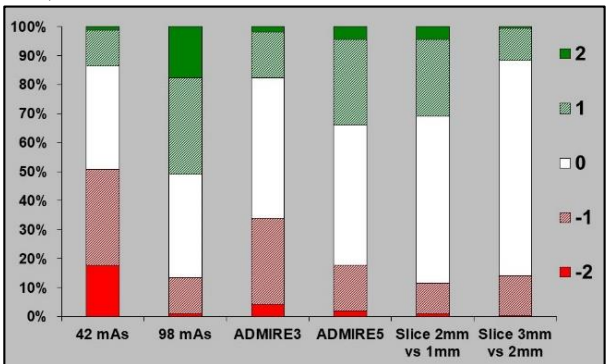

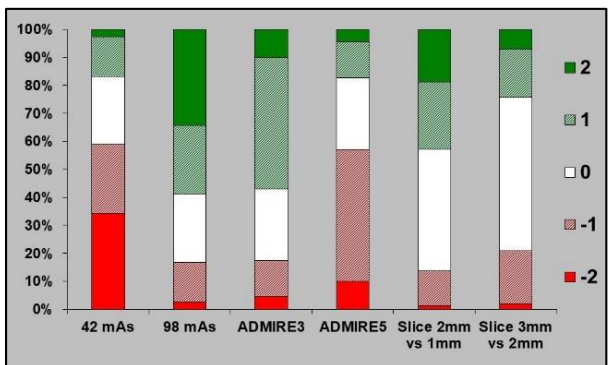

Figure 3 Favorable vs. unfavorable scores for image quality presented as percentage of scores assigned to the current image type when compared to other image types. Compared to the alternative image, the current image was rated as follows; score 2 as superior, score 1 as probably superior, score 0 as equivalent, score -1 as probably inferior, and score -2 as inferior for image criteria a) Visually sharp reproduction of the liver parenchyma b) Visually sharp reproduction of the pancreas contours c) Visually sharp reproduction of the kidneys and proximal ureters d)

Reproduction of lymph nodes $<15 \mathrm{~mm}$ in diameter e) Overall image quality for diagnostic purposes. 


\section{Effect of iterative reconstruction algorithm}

Using ADMIRE strength 5 instead of 3 resulted in significantly better image quality for Criteria

C3 (Kidneys and proximal ureters, $p<0.01$ ) and C4 (Lymph nodes, $p<0.001$ ), but significantly inferior for Criteria C1 (Liver parenchyma) and C5 (Overall image quality both $p<0.001)$. For criterium C2 (Pancreas contour), there was no statistical difference between AD5 and AD3 (Table 3).

Table 3. Visual Grading Regression (VGR) coefficients for all criteria assessed in a comparison of MPR images reconstructed with two ADMIRE strengths and three slice thicknesses.

\section{Regression coefficients}

\begin{tabular}{|c|c|c|c|c|c|}
\hline Criterion & $\begin{array}{c}\log \\
(\mathbf{m A s})\end{array}$ & $\begin{array}{c}\text { Reconstruction } \\
\text { algorithm } \\
\text { ADMIRE } 5 \\
\text { vs. } \\
\text { ADMIRE } 3 \\
\end{array}$ & $\begin{array}{c}\text { Slice } \\
\text { thickness } \\
2 \mathrm{~mm} \\
\text { vs. } \\
1 \mathrm{~mm} \\
\end{array}$ & $\begin{array}{c}\text { Slice } \\
\text { thickness } \\
3 \mathbf{~ m m} \\
\text { vs. } \\
1 \mathrm{~mm} \\
\end{array}$ & $\begin{array}{c}\text { Slice } \\
\text { thickness } \\
3 \mathrm{~mm} \\
\text { vs. } \\
2 \mathrm{~mm} \\
\end{array}$ \\
\hline $\begin{array}{l}\text { C1 Visual sharp reproduction } \\
\text { of liver parenchyma }\end{array}$ & $1.25 * * *$ & $-1.35 * * *$ & $0.49 * * *$ & $0.53 * * *$ & $0.04^{\circ}$ \\
\hline $\begin{array}{l}\text { C2 Visual sharp reproduction } \\
\text { of the pancreas contour }\end{array}$ & $1.75 * * *$ & $0.05^{\circ}$ & $0.47 * * *$ & $0.50 * * *$ & $0.03^{\circ}$ \\
\hline $\begin{array}{l}\text { C3 Visual sharp reproduction } \\
\text { of the kidneys and } \\
\text { proximal ureters }\end{array}$ & $1.78 * * *$ & $0.21 * *$ & $0.55 * * *$ & $0.55 * * *$ & $0.004^{\circ}$ \\
\hline $\begin{array}{l}\text { C4 Reproduction of lymph } \\
\text { nodes }<15 \mathrm{~mm} \text { in diameter }\end{array}$ & $1.55 * * *$ & $0.48 * * *$ & $0.51 * * *$ & $0.49 * * *$ & $-0.02^{\circ}$ \\
\hline $\begin{array}{l}\text { C5 Overall image quality for } \\
\text { diagnostic purposes }\end{array}$ & $1.65 * * *$ & $-0.86 * * *$ & $0.71 * * *$ & $0.87 * * *$ & $0.16^{\circ}$ \\
\hline
\end{tabular}

***) $\left.p<0.001 ; * *) p<0.01 ;^{\circ}\right)$ not significant

\section{Effect of slice thickness}

Independent of the algorithm strength, better image quality was observed for all image criteria in favor of slice thicknesses of $2 \mathrm{~mm}$ and $3 \mathrm{~mm}$ compared to $1 \mathrm{~mm}(p<0.001)$ (Table 3$)$. The potential dose reductions with an increase in slice thickness from $1 \mathrm{~mm}$ to $2 \mathrm{~mm}$ and $1 \mathrm{~mm}$ to $3 \mathrm{~mm}$ ranged from $24 \%$ to $35 \%$ and $25 \%$ to $41 \%$, respectively (Table 5). No significant differences in image quality were found between $3 \mathrm{~mm}$ and $2 \mathrm{~mm}$ slice thicknesses (Table 3 ). 
Separate comparisons of the effect of AD3 and AD5 at the same slice thickness (Table 4A-C) resulted in minor deviations from the pattern described above. It should also be noted that with increasing slice thickness, the effects of tube load, and in most cases also reconstruction algorithm, were weaker (regression coefficients closer to 0), although still strongly significant.

Table 4 A-C Visual Grading Regression (VGR) coefficients for all criteria assessed in a comparison of images reconstructed with two reconstruction algorithms, analyzed separately for comparisons involving only one slice thickness.

\section{A. Comparison between ADMIRE 5 vs. ADMIRE 3 at $1 \mathrm{~mm}$ slice thickness}

\section{Regression coefficients}

\section{Reconstruction algorithm} ADMIRE 5

VS.

\section{Criterion}

$\log (\mathbf{m A s})$

C1 Visual sharp reproduction of liver parenchyma

C2 Visual sharp reproduction of pancreas contours

C3 Visual sharp reproduction of kidneys \& proximal ureters

C4 Reproduction of lymph nodes $<15 \mathrm{~mm}$ in diameter

C5 Overall image quality for diagnostic purposes
$2.06 * * *$

$2.39 * * *$

$2.47 * * *$

$2.22 * * *$

$2.38 * * *$
ADMIRE 3

$$
-0.55 * * *
$$

$0.54 * * *$

$0.63 * * *$

$0.87 * * *$

$-0.26^{*}$

***) $p<0.001 ; *) p<0.05$

\section{B. Comparison between ADMIRE 5 vs. ADMIRE 3 at $2 \mathrm{~mm}$ slice thickness}

\section{Regression coefficients}

Reconstruction algorithm ADMIRE 5

\section{Criterion}

C1 Visual sharp reproduction of liver parenchyma

C2 Visual sharp reproduction of pancreas contours

C3 Visual sharp reproduction of kidneys \& proximal ureters

C4 Reproduction of lymph nodes $<15 \mathrm{~mm}$ in diameter

C5 Overall image quality for diagnostic purposes

$\log (\mathbf{m A s})$

$0.93 * * *$

$1.35^{* * * *}$

$1.25 * * *$

$1.24 * * *$

$1.30 * * *$
VS.

ADMIRE 3

***) $p<0.001 ; * *) p<0.01 ;^{\circ}$ ) not significant 
Regression coefficients

Reconstruction algorithm

ADMIRE 5

\section{Criterion}

C1 Visual sharp reproduction of liver parenchyma

C2 Visual sharp reproduction of pancreas contours

C3 Visual sharp reproduction of kidneys \& proximal ureters

C4 Reproduction of lymph nodes $<15 \mathrm{~mm}$ in diameter

C5 Overall image quality for diagnostic purposes vs.

\section{$\log (\mathbf{m A s})$}

$0.51 * *$ ADMIRE 3

$0.87 * * *$

$-1.82 * * *$

$0.93 * * *$

$0.93 * * *$

$-0.26^{*}$

$0.72 * * *$

$-0.16^{\circ}$

$0.00^{\circ}$

$0.94 * * *$

***) $p<0.001 ; * *) p<0.01 ; *) p<0.05 ;^{\circ}$ ) not significant

\section{Interobserver reliability}

The inter-observer reliability expressed as weighted agreement ranged from 0.720 to 0.782 with weighted kappa values significantly greater than 0 , showing a moderate agreement among the observers.

\section{Objective assessment}

\section{Effect of tube load, reconstruction algorithm and slice thickness}

Noise (SD) decreases with increase in tube load and with an increase in strength from AD3 to AD5 compared to filtered Back Projection (FBP) (Figure 4). Consequently, the CNR increases with an increase in tube load and an increase in strength from AD3 to AD5 compared to FBP (Figure 5). 
Table 5. Estimates of potential dose reductions for variation in slice thickness from $1 \mathrm{~mm}$ to $2 \mathrm{~mm}$ and $1 \mathrm{~mm}$ to $3 \mathrm{~mm}$ independent of the ADMIRE algorithm strength.

\section{Estimated dose reduction ( $95 \%$ confidence limits)}

\section{Criterion}

C1 Visual sharp reproduction of liver parenchyma

C2 Visual sharp reproduction of pancreas contours

C3 Visual sharp reproduction of kidneys \& proximal ureters

C4 Reproduction of lymph nodes $<15 \mathrm{~mm}$ in diameter

C5 Overall image quality for diagnostic purposes

\begin{tabular}{cc}
\hline $\begin{array}{c}\text { Slice thickness } \\
\text { 2 mm vs. 1 mm }\end{array}$ & $\begin{array}{c}\text { Slice thickness } \\
\text { 3 mm vs. 1 mm }\end{array}$ \\
\hline $32 \%(22 \% ; 43 \%)$ & $35 \%(21 \% ; 48 \%)$ \\
$24 \%(16 \% ; 32 \%)$ & $25 \%(15 \% ; 35 \%)$ \\
$26 \%(19 \% ; 34 \%)$ & $27 \%(16 \% ; 37 \%)$ \\
$28 \%(19 \% ; 37 \%)$ & $27 \%(15 \% ; 39 \%)$ \\
$35 \%(27 \% ; 43 \%)$ & $41 \%(31 \% ; 50 \%)$ \\
\hline
\end{tabular}

a)

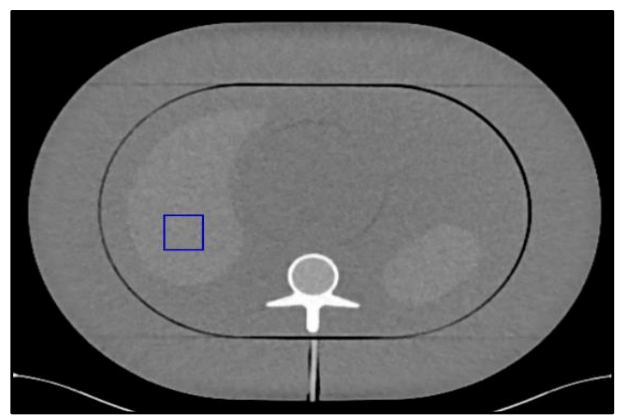

c)

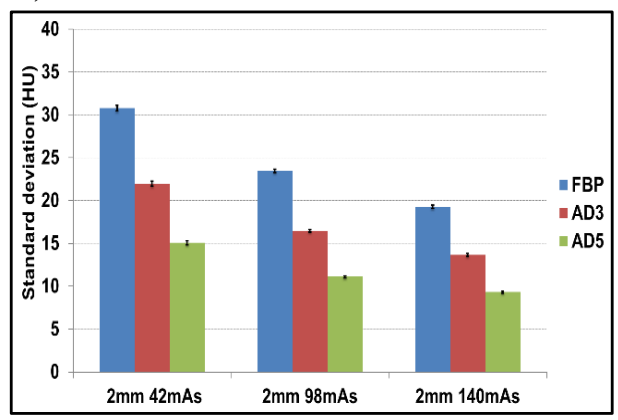

b)

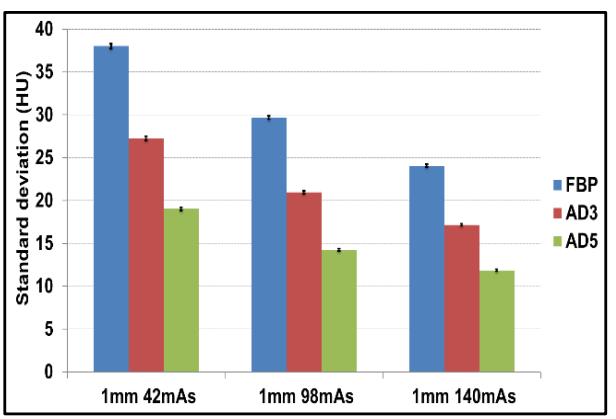

d)

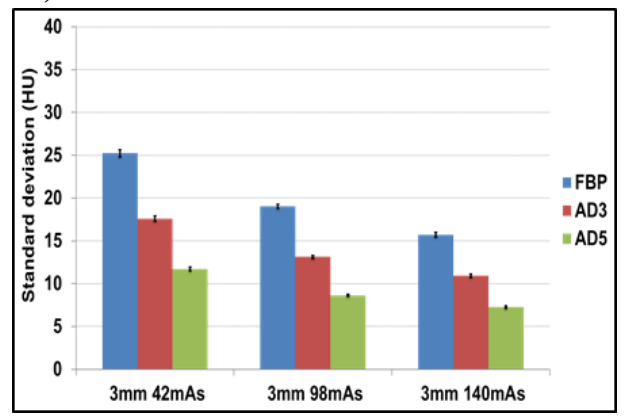

Figure 4 Effect of tube load, reconstruction algorithms ADMIRE strength 3 (AD3), strength 5 (AD5), filtered back projection (FBP) and slice thickness on noise estimated as standard deviation (SD) (a) Phantom image showing ROI placement in the liver section, with SD variation at slice thickness $1 \mathrm{~mm}$ (b), $2 \mathrm{~mm}$ (c) and $3 \mathrm{~mm}$ (d). The error bars represent the $95 \%$ confidence interval. 
a)

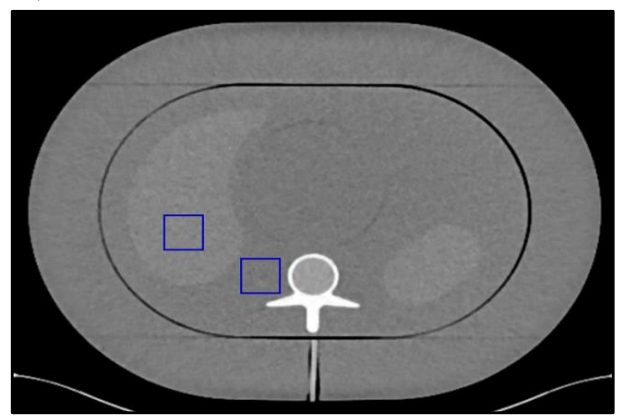

c)

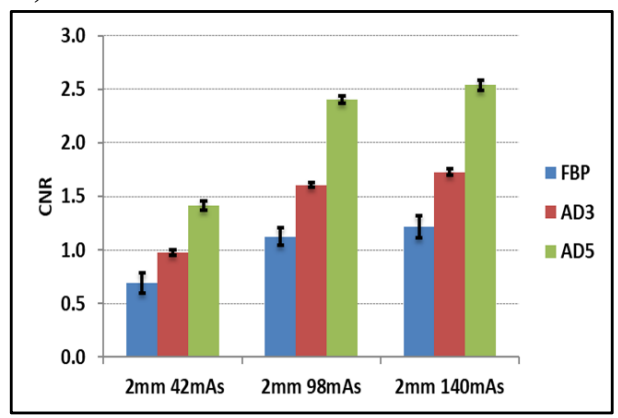

b)

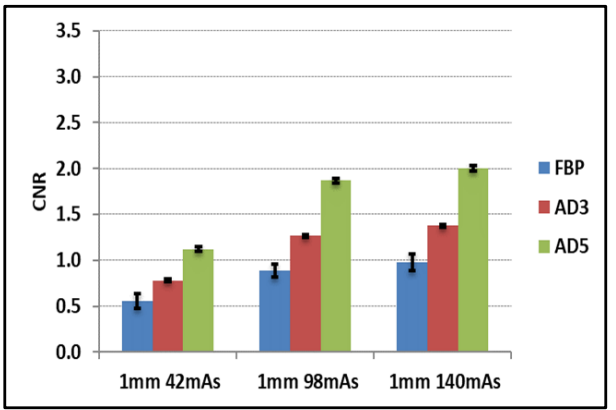

d)

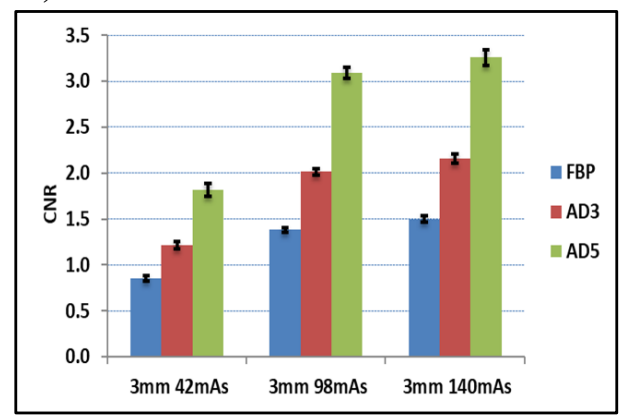

Figure 5 Effect of tube load, reconstruction algorithm and slice thickness on contrast-to-noise ratio (CNR) in the liver of an anthropomorphic abdominal phantom. Phantom image showing ROI placements in the liver section and adjacent background material just outside the liver (a), variation in CNR at slice thickness at $1 \mathrm{~mm}$ (b), $2 \mathrm{~mm}$ (c) and 3mm (d). AD3 and AD5 represents ADMIRE strengths 3 and 5 respectively and FBP represents filtered back projection. The error bars represent the $95 \%$ confidence interval.

\section{Noise-Power Spectrum (NPS)}

The noise-power decreases with increasing ADMIRE strength compared to FBP. The decrease in noise power is more pronounced at the higher spatial frequencies when using AD3 and particularly with AD5 compared to FBP (Figure 6) as shown by the dotted vertical lines indicating the median frequency values. 
a)

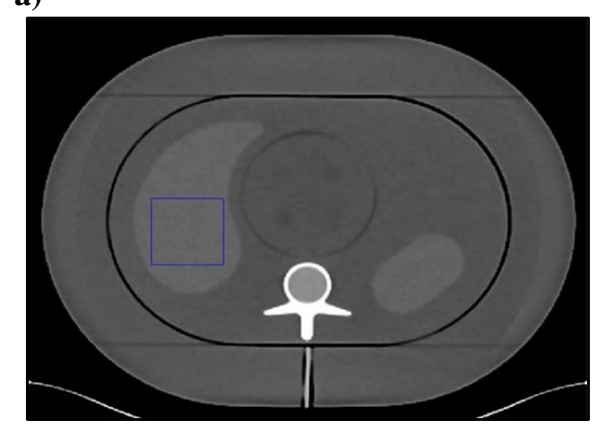

b)

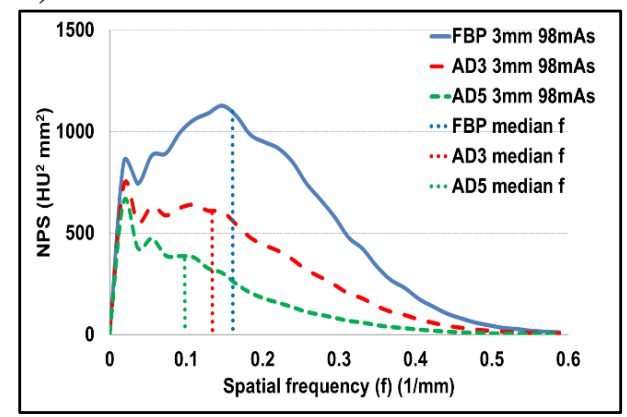

c)

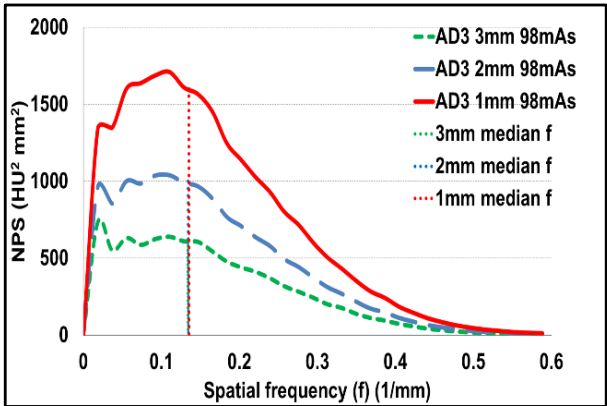

Figure 6 Effect of reconstruction algorithm and slice thickness on noise power spectrum (NPS); (a) phantom image showing ROI placement (b) comparison between ADMIRE strengths 3 and 5 and filtered back projection (FBP) at $98 \mathrm{mAs}$ and $3 \mathrm{~mm}$ slice thickness (c) NPS comparison between three slice thicknesses for ADMIRE strength 3 at 98 mAs. The vertical dotted lines indicate median spatial frequency (f) values in (b) and (c).

\section{Discussion}

Results of performance studies of MBIR that measure visual and objective image quality using a phantom [6] are limited to specific conditions and assumptions. But, if quantitative phantom measurements agree with visual assessment of image quality in clinical examinations, a decision on the desired clinical image quality can be reached for successful implementation of the algorithm in clinical practice $[9,19]$. We performed an observer evaluation study combined with objective phantom measurements to estimate the potential dose reduction with the intention of obtaining information about the clinical utility of the ADMIRE algorithm.

In the present study, delineation of the liver parenchyma and overall image quality assessment was graded as being inferior for AD5, independent of the slice thickness, and did not allow for 
any potential dose reduction in concurrence with Kataria et al [5]. As noise reduction in IR is a nonlinear process, the loss of image sharpness is usually related to the alteration of noise texture in the images as determined by the shape and magnitude of the NPS curve [7]. Dose reduction is exceedingly dependent on the contrast of the object, with higher reductions possible when assessing high-contrast objects compared to low-contrast objects such as the liver parenchyma $[8,20]$.

The ADMIRE NPS from our study show lower magnitude and a shift towards lower spatial frequencies for strengths 3 and 5 compared to FBP. An explanation for this could be that the ADMIRE algorithm analyses noise in a larger environment (voxel) for better separation of noise from the anatomical structures, which could possibly help retain a similar noise texture in the images [9, 20,21], unlike other MBIR algorithms where the NPS curve differed more from that of FBP [22]. However, the left shift of the NPS curve for AD3 and AD5 compromises the lowcontrast resolution and could contribute to the unusual appearance of the images [20] as was the case with our observers who were critical about the unusual appearance of AD5 images during the coaching session.

There is a variability in visual perception among the radiological community, but to an increase the diagnostic confidence, a certain degree of noise in the images is necessary to accentuate the sharpness in the image [20]. Schaller et al [7] and Gordic et al [9] found no significant differences in image quality between AD3 and AD5, when studying the effect of ADMIRE on contrast-enhanced images. Perhaps lower $\mathrm{kV}$ settings and contrast enhancement can partly explain the improvement in image quality for AD5 in those studies as both parameters affect image quality. The present study showed that AD3, on the other hand, renders significantly improved image quality when considering all of the criteria assessed, despite lower noise reduction (Figure 6), compared to AD5 with improved image quality for only two out of five image criteria. This is in concurrence with Ellman et al [8] who found no significant differences 
in potential dose reductions between AD3 and AD5 despite the significant reduction in image noise in all anatomical contrast sub-groups studied.

In the present study, Criterion 1 (liver parenchyma) and Criterion 5 (overall image quality), showed consistently inferior image qualities for AD5 across all slice thicknesses. A slight improvement in image quality for three out of five criteria in favor of the higher strength at $1 \mathrm{~mm}$ slice thickness was observed, when comparing the two algorithm strengths at same slice thickness. This could be attributed to the reduction in partial volume averaging [11] and/or increase in quantum noise due to Poisson distribution in thinner slices [23]. Contrary to our study, two studies have reported retained image quality with thin slices for the higher strength of another MBIR [11, 12]. Both these studies evaluated the effect of slice thickness using iterative model-based reconstruction (IMR) in brain CT and abdominal phantom respectively. However, the results of the present study are specific for ADMIRE and the Siemens Force scanner as the performance of IR is vendor specific and cannot generally be transferred from one system or vendor to another.

The added diagnostic value of MPR reconstructions has previously been studied in suspected appendicitis patients [13] and detection of lung nodules [24]. However, the simultaneous comparison of MPR images in our study showed no improvement in image quality for AD5 and Criteria 1 and 5 as has also been found previously for axial comparisons [5].

A strength of the present study is that two dose levels for each patient were obtained with a single acquisition, thus avoiding the ethical dilemma of multiple acquisitions of the same anatomical area and patient as well as obtaining comparable images for both levels in the same breath hold. The pairwise MPR observer evaluation also replicates the clinical setting providing a direct visual impression of which image appears better and could possibly have an impact in determining the clinically acceptable image quality. The visual grading regression methodology 
moreover, provides a unique estimate of possible dose reductions for comparable measures of image quality.

There are some limitations in our study. First, despite the randomization of the images, it is impossible to completely blind the observers to the differences in image texture and variation in number of slices. Secondly, we did not cross-compare thick slices of AD3 with thin slices of AD5, this would have been valuable in evaluating image quality for thinner slices of AD5 compared to the clinical standard of 3mm slice thickness for AD3. We used fixed tube voltage at $120 \mathrm{kV}$ for the study protocol but automated $\mathrm{kV}$ setting ("Care $\mathrm{kV}$ ") is used in the clinical routine, this could affect the dose reduction possibilities in a clinical setting.

\section{Conclusion}

As expected, when increasing slice thickness from $1 \mathrm{~mm}$ to $2 \mathrm{~mm}$ or $3 \mathrm{~mm}$, independent of the IR algorithm strength, a dose reduction is possible. AD5, in comparison to AD3, has diverse effects on the five image quality criteria depending on slice thickness and further dose reductions are limited to certain criteria. Our findings do not support a general recommendation to replace AD3 by AD5 in clinical abdominal CT protocols. 


\section{References}

[1] ICRU report No. 87, Radiation dose and image quality assessment in Computed Tomography, J ICRU 12 (2012):1-149.

[2] The LOCAT Group, Low-dose CT for the diagnosis of appendicitis in adolescents and young adults (LOCAT): a pragmatic, multicentre, randomised controlled non-inferiority trial, Lancet Gastroenterol Hepatol 2 (2017):793-804.

[3] M. Kalra, S.D. Sodickson, W.W. Mayo-Smith, CT radiation key concepts for gentle and wise use. Radiographics 35 (2015):1707-21.

[4] L. Liu, Model-based Iterative Reconstruction A Promising Algorithm for Today's CT imaging, J Med Radiat Sci 45 (2014):131-6.

[5] B. Kataria, J. Nilsson Althén, Ö. Smedby, A.Persson, H. Sökjer, M. Sandborg, Assessment of image quality in abdominal CT: potential dose reduction with modelbased iterative reconstruction, Eur Radiol 28 (2018):2464-73.

[6] J. Solomon, A. Mileto, J.C. Ramirez-Giraldo, E. Samei, Diagnostic performance of an Advanced Modeled Iterative Reconstruction Algorithm for low-contrast detectability with a third-generation dual-source multidetector CT scanner: Potential for radiation dose reduction in a multireader study, Radiology 275 (2015):735-45.

[7] F. Schaller, M. Sedlmair, R. Raupach, M. Uder, M. Lell, Noise Reduction in Abdominal Computed Tomography Applying Iterative Reconstruction (ADMIRE), Acad Radiol 23 (2016): 1230-38.

[8] S. Ellmann, F. Kammerer, T. Allmendinger, et al, Advanced Modeled Iterative Reconstruction (ADMIRE) facilitates radiation dose reduction in abdominal CT, Acad Radiol 25 (2018):1277-84.

[9] S. Gordic, L. Desboilles, P. Stolzmann, et al, Advanced modelled iterative reconstruction for abdominal CT: qualitative and quantitative evaluation, Clin Radiol 69 (2014):497-504. 
[10] C. Falck, M. Galanski, H. Shin, Sliding thin slag averaging for improved depiction of low-contrast lesions with radiation dose savings at thin-section CT, Radiographics 30 (2010): 317-26.

[11] M.L. Aurumskjöld, K. Ydström, A. Tingberg, M. Söderberg, Model-Based Iterative Reconstruction enables the evaluation of thin-slice Computed Tomography images without degrading image quality or increasing radiation dose, Radiat Prot Dosimetry 169(2016):100-6.

[12] T. Inoue, T. Nakaura, M. Yoshida, et al, Brain computed tomography using iterative reconstruction to diagnose acute middle cerebral artery stroke: usefulness in combination of narrow window setting and thin slice reconstruction, Neuroradiology 60(2018): 373-9.

[13] H.C. Kim, D.M. Yang, W. Jin, S.J. Park, Added diagnostic value of multiplanar reformation of Multidetector CT data in patients with suspected appendicitis, Radiographics 28(2008):393-406.

[14] Report of AAPM task group 204, Size-specific dose estimates in paediatric and adult body CT examinations, (2011) https://www.aapm.org/pubs/reports/RPT_204.pdf . (accessed 6 June 2019).

[15] European Commission EU publications, European guidelines on quality criteria for computed tomography, 2000 https://publications.europa.eu/en/publication-detail//publication/d229c9e1-a967-49de-b169-59ee68605f1a/language-en (accessed 10 December 2018).

[16] F.R. Verdun, D. Racine, J.G. Ott, et al, Image quality in CT: From physical measurements to model observers, Phys Med 31(2015):823-43.

[17] Ö. Smedby, M. Fredrikson, Visual grading regression: analysing data from visual grading experiments with regression models, Br J Radiol 83 (2010):767-75. 
[18] Ö. Smedby, M. Fredrikson, J. De Geer, L. Borgen, M. Sandborg, Quantifying the potential for dose reduction with visual grading regression, Br J Radiol 86(2013):1-6.

[19] H.M. Whitson, Quantifying differences in CT image quality between a MBIR algorithm, an Adaptive statistical iterative reconstruction algorithm and Filtered back projection Scholar archive 3913, (2017) http://digitalcommons.ohsu.edu/etd/3913 (accessed 1 December 2018).

[20] E.C. Ehman, L.Yu, A. Manduca, et al, Methods for clinical evaluation of noise reduction techniques in abdominopelvic CT, RadioGraphics 34 (2014):849-62.

[21] K.M. Kanal, B.K. Stewart, O. Kolokythas, W.P. Shuman, Impact of operator selected image noise index and reconstruction slice thickness on patient radiation dose in 64MDCT, AJR Am J Roentgenol 189 (2007):219-25.

[22] A. Love, M.L. Olsson, R. Siemund, F. Stalhammar, I.M. Bjorkman-Burtscher, M. Söderberg, Six iterative reconstruction algorithms in brain CT: a phantom study on image quality at different radiation dose levels, Br J Radiol 86(2013):20130388.

[23] J.C. Ramirez-Giraldo, K.L Grant, R. Raupach, White paper ADMIRE - Advanced modelled iterative reconstruction, (2018) https://www.healthcare.siemens.com/computed-tomography/technologiesinnovations/admire (accessed 6 December 2018).

[24] S. Matsumoto, Y. Ohno, H. Yamagata, M. Nogami, A. Kono, K. Sugimura, Potential contribution of multiplanar reconstruction (MPR) to computer-aided detection of lung nodules on MDCT, Eur J Radiol 81 (2012):366-70. 


\section{FACULTY OF MEDICINE AND HEALTH SCIENCES}

Linköping University Medical Dissertation No. 1683, 2019

Department of Medical and Health Sciences

Linköping University

SE-581 83 Linköping, Sweden

wwW.liu.se

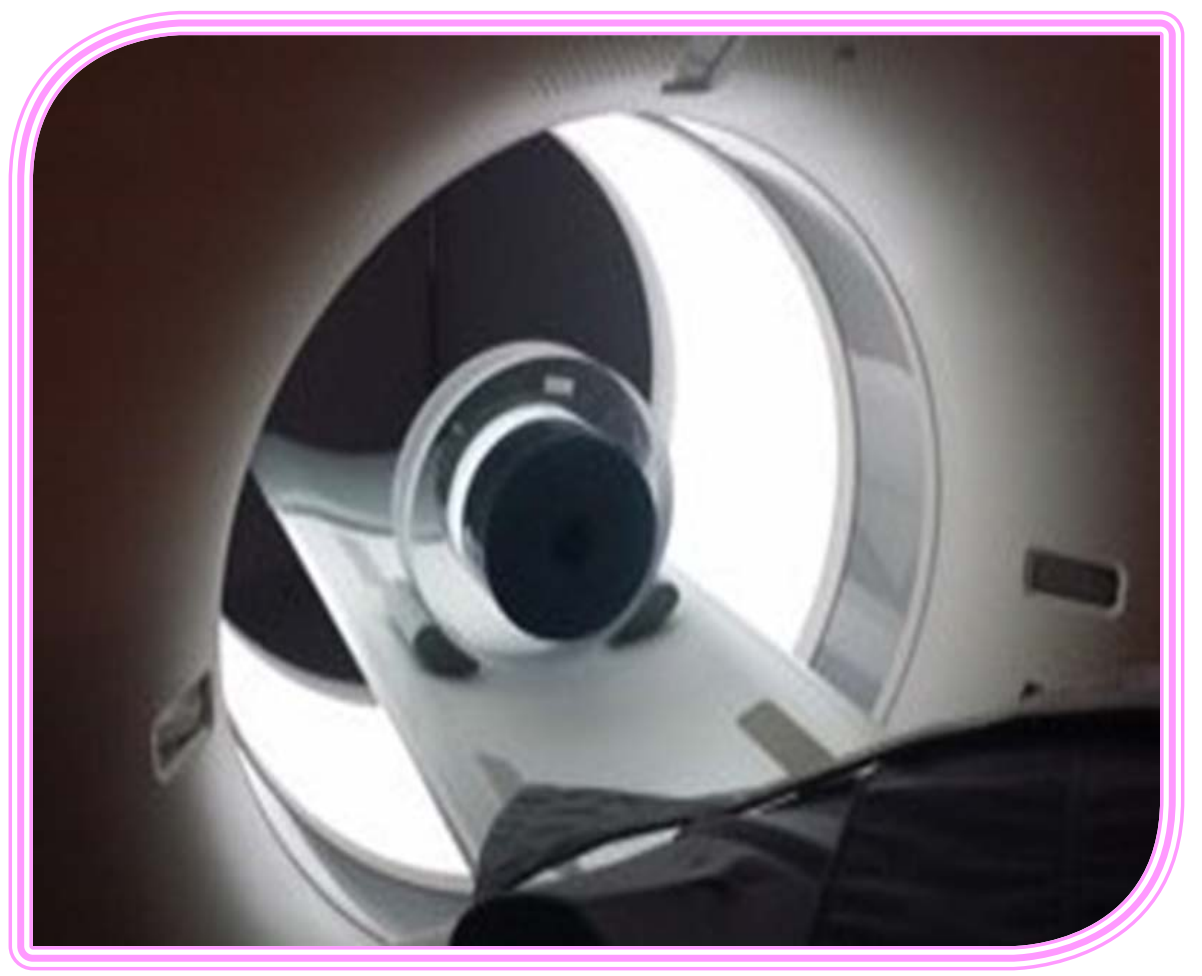

